
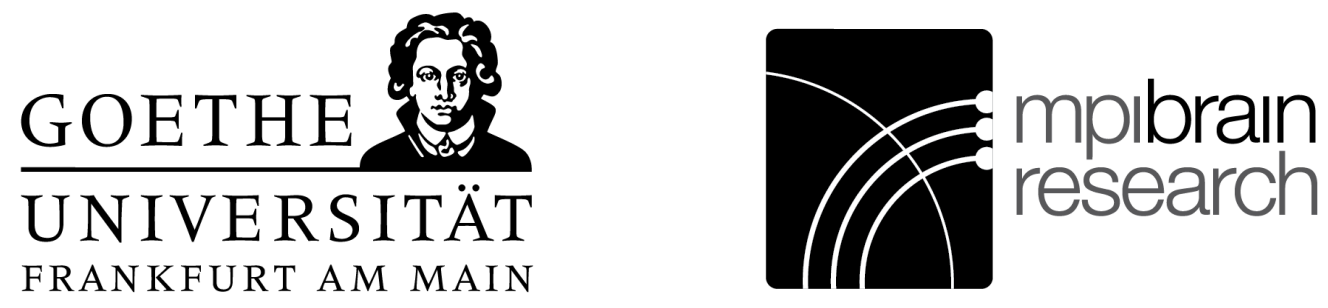

\title{
Exploring the in vivo subthreshold membrane activity of phasic firing in midbrain dopamine neurons
}

\author{
A dissertation for a doctorate degree in natural sciences \\ submitted to the Faculty of Biosciences of Goethe University
}

by

\section{Kanako Otomo}

from Tokyo, Japan

Frankfurt am Main, 2021 

Submitted to the Faculty of Biosciences of Goethe University as a PhD dissertation

Dean: Prof. Dr. Sven Klimpel

Evaluators: Prof. Dr. Manfred Kössl Prof. Dr. Gilles Laurent Prof. Dr. Amparo Acker-Palmer Prof. Dr. Henner Hollert

Date of disputation: 24 June 2021 

The work presented in this dissertation was published in Nature Communications:

*Otomo, K., *Perkins, J., Kulkarni, A., Stojanovic, S., Roeper, J., Paladini, C. (2020) In vivo patch-clamp recordings reveal distinct subthreshold signatures and threshold dynamics of midbrain dopamine neurons. Nature Communications. 11,6286. *Shared first authors 

In memory of Professor Dr. Alexandre Dayer 



\section{ACKNOWLEDGMENTS}

I would like to take this opportunity to express my sincere appreciation for the people who have assisted and supported me during my PhD. First of all, completion of this dissertation would not have been possible without the extensive support I received from the International Max Planck Research School/Max Planck Institute for Brain Research (MPIBR), particularly during the final years of my PhD. I would thus like to express my utmost gratitude to Prof. Dr. Gilles Laurent and Dr. Irina Epstein at MPIBR for their support, mentorship and patience, and for paving the way for me to finish my degree. I am extremely grateful to have Prof. Dr. Manfred Kössl and Dr. Hiroshi Ito in my Thesis Advisory Committee and as my supervisors, who readily accommodated my needs and provided scientific and administrative support. I am lucky to have moved to Frankfurt around the same time as Hiroshi, which allowed me to have him as a mentor and friend since the beginning. I would like to express my gratitude to my $\mathrm{PhD}$ adviser Prof. Dr. Jochen Roeper for providing me with the opportunity to work on this project.

I would like to further thank my colleagues at the Institute of Neurophysiology for their help, support, and friendship for the last five years. It has been an utter pleasure to have Josef Shin around from the beginning of this journey, who was always a reliable, honest, hilarious, and brilliant friend and colleague. His objective and impartial opinions, deep philosophical insights, along with his scientific feedback (especially for my dissertation), were truly invaluable. I am also grateful to be able to call Dr. Kauê Costa a friend, colleague, and now a (de facto) mentor. I am fond of how our friendship has matured over the years and that I now take comfort in his -at times merciless- honesty and constructive criticism. He helped me immensely with my dissertation and also provided the initial code for anatomical mapping. I also want to thank Navid Farassat for teaching me in vivo extracellular recording/juxtacellular labeling and retrograde infusion, and Strahinja Stojanovic for his help on analysis, who provided the initial data extraction, Gaussian fitting, and clustering scripts. Special thanks go to Ximena SalinasHernández and Pascal Vogel for friendly discussions and being all around kind and caring.

Regina Giegerich gave me enormous support in and outside of the lab, administrative and otherwise, for which I am grateful. I want to further extend my appreciation to the institute technicians Jasmine Sonntag, Beatrice Fischer, and Felicia 
Müller-Braun, who maintained the lab in a superb condition and provided numerous support for immunohistochemistry; to the members of the institute workshop Thomas Wulf, Hans-Peter Günster, and Charly Winter, who built modules and apparatuses necessary for my experiments and provided IT assistance; to the animal caretaker Günther Amrhein for properly caring and maintaining my animals; and to the institute PIs Dr. Torfi Sigurdsson and Dr. Sevil Duvarci for their encouragement, support, and feedback on my project.

Additionally, I want to thank Maren Baier and Dr. Arjan Vinck from MPIBR for their administrative assistance regarding immigration and university registration, which allowed me to focus on research. I also want to thank Dr. Christine Reif-Leonhard and Ms. Kirsten Wischnewski for helping me get back on my feet.

I have been extremely fortunate to have such wonderful, genuine people in my life; most of whom live far away, but without whose friendship and support, I would not have survived my $\mathrm{PhD}$ and navigated through to where I am now. My daily chat with Mika Yokoo and Naoko Kasuga kept me sane and grounded during the most difficult times as well as during the pandemic. To have such a safe place where we can discuss anything from political affairs to absolute nonsense to personal struggles is a gift that I treasure. Gregorio Galinãnes, Claudia Bonardi, and Karin Morandell: thank you for creating a warm and fun home to visit and recharge. It was your support, empathy, and encouragement that got me through many hardships, and I miss Le Cheval Blanc dearly. Genevieve Artadi: you are my inspiration in life and music. Our every random rendez-vous in Europe restored my passion and drive to continue research. John Michael Richardson: thank you for still being a part of my life and being open to sharing your unique take on political and philosophical subjects all the way from the PST zone, as well as for proofing my dissertation. Joni Schukowski: I am grateful we have found each other in the midst of chaos and that we understand each other on a deep level. Note, Hiroko, Asuhi, Sayoko, and Saori: Thank you for being my (de facto) family. Special thanks go to The Dream Team/Dayer Lab for continued fun times, and to Prof. Dr. Daniel Huber for being my mentor at crucial moments. Lastly, I would not have made it this far if it was not for Lorenz Marschalek's support, who stayed by my side at every critical moment and whenever things got rough. Thank you. 


\section{TABLE OF CONTENTS}

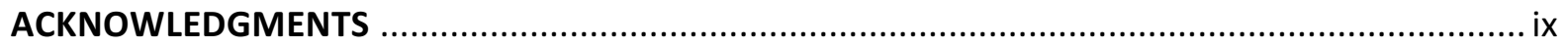

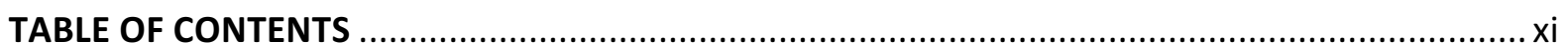

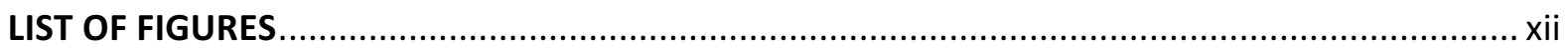

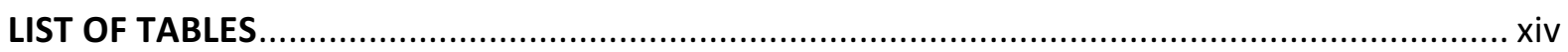

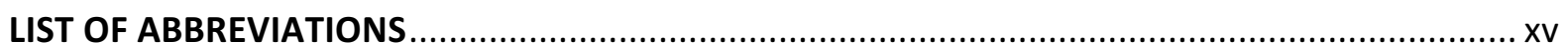

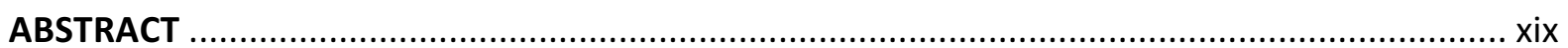

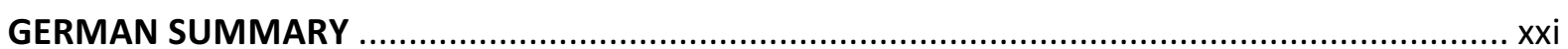

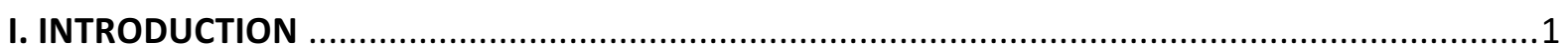

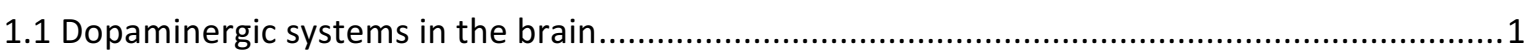

1.1.1 Molecular pathways of the dopaminergic system ....................................................

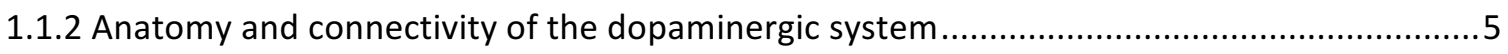

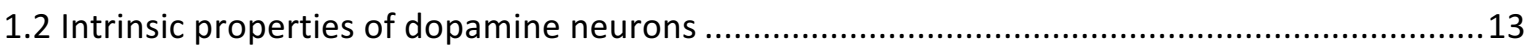

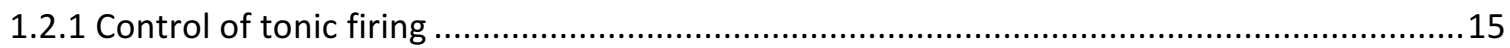

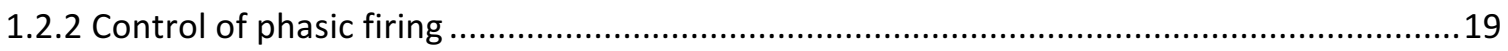

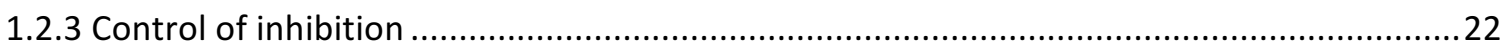

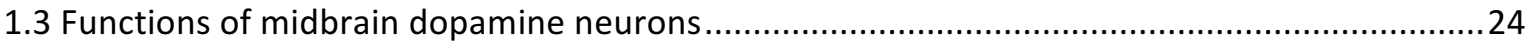

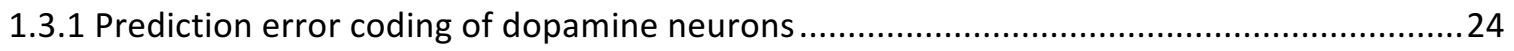

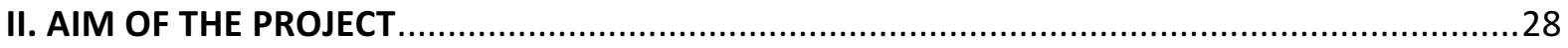

III. MATERIALS \& METHODS

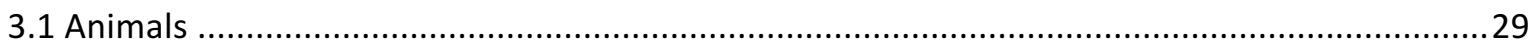

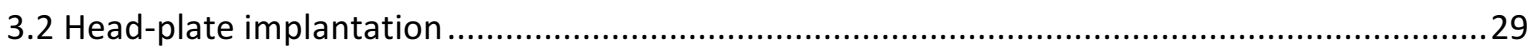

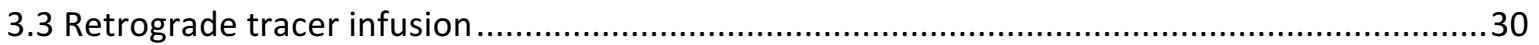

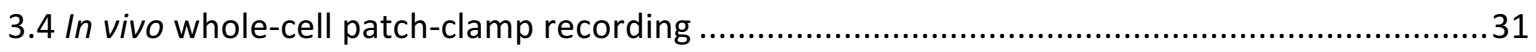

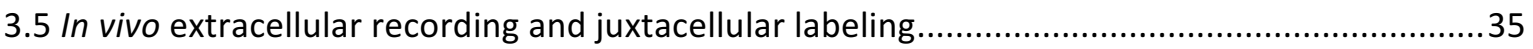

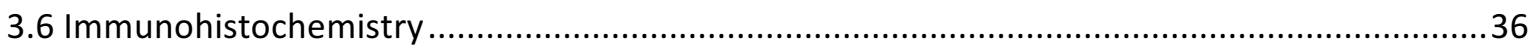

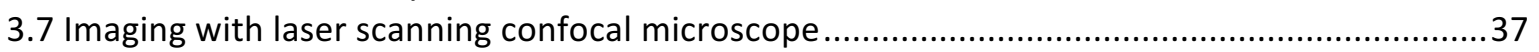

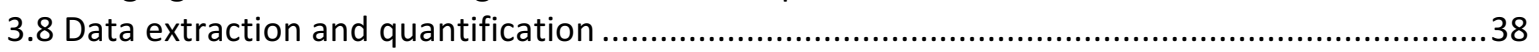

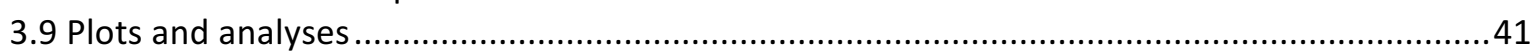

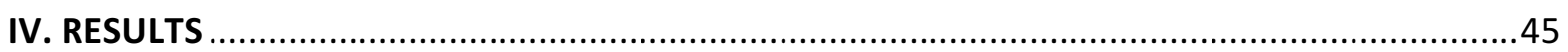

Exploring the subthreshold membrane potentials of midbrain dopamine neurons in vivo .............45

4.1 Establishing the deep-brain whole-cell patch-clamp recording method .................................45

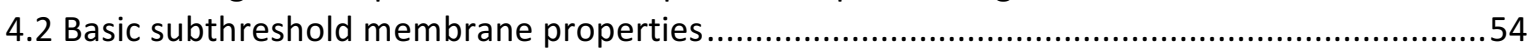

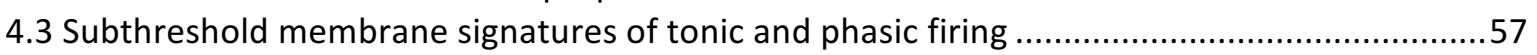

4.4 Characterization of subthreshold membrane behaviors of the population .............................. 74

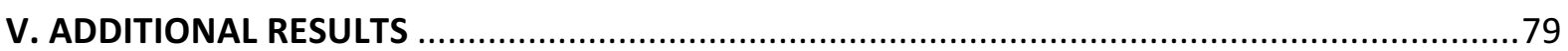

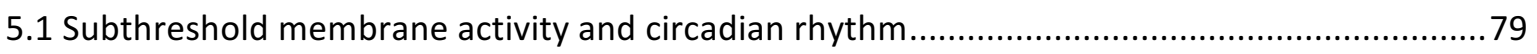

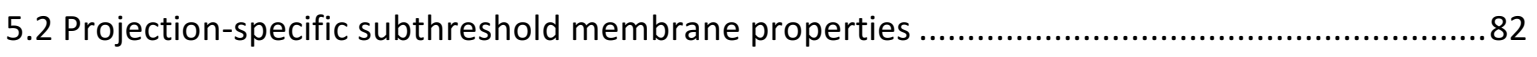

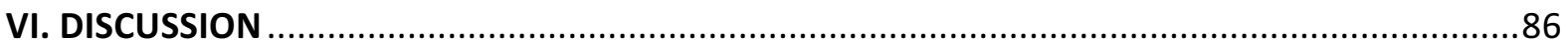

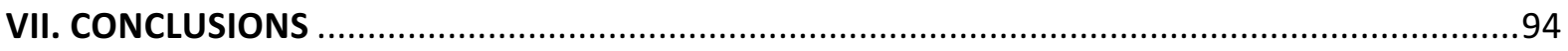

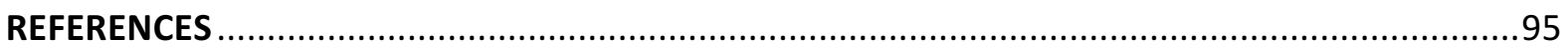




\section{LIST OF FIGURES}

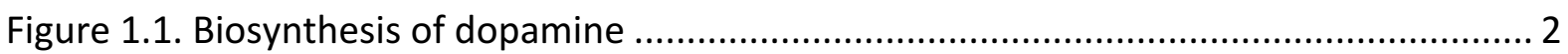

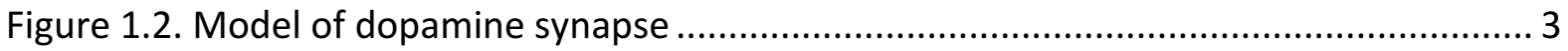

Figure 1.3. Brain regions involved in the cortico-basal ganglia-thalamo-cortical loop ........... 6

Figure 1.4. Inputs and outputs of dopamine neurons...................................................... 7

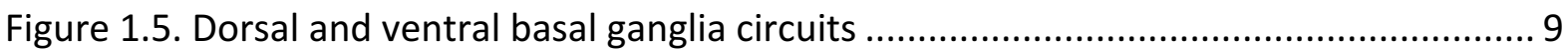

Figure 1.6. Action potential waveforms of dopamine and GABAergic neurons in substantia

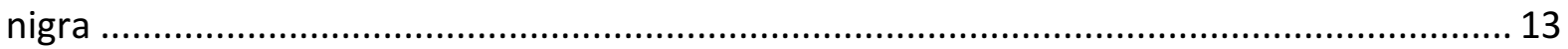

Figure 1.7. Tonic "pacemaker" firing \& phasic "burst" firing of dopamine neurons............. 14

Figure 1.8. Voltage-dependent L-type $\mathrm{Ca}^{2+}$ channels drive pacemaker oscillations in

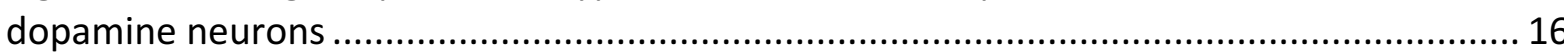

Figure 1.9. Sag component displayed by in vitro dopamine neuron upon hyperpolarization.

Figure 1.10. Blocking T-type $\mathrm{Ca}^{2+}$ channels induce burst firing of in vitro dopamine neurons

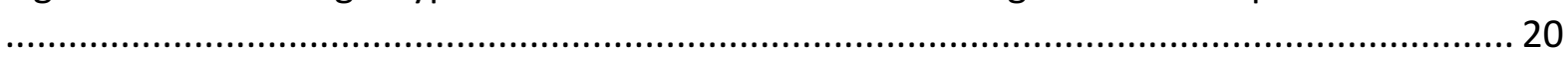

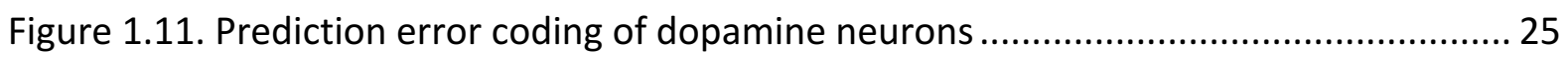

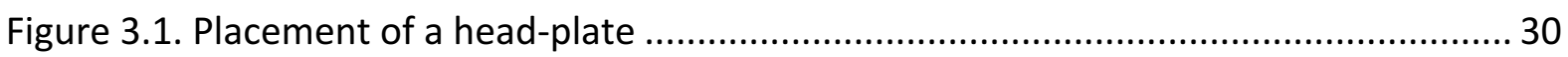

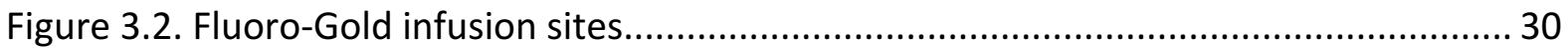

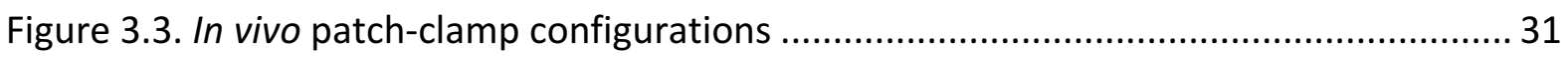

Figure 3.4. In vivo activity of dopamine neuron recorded in the whole-cell configuration ... 33

Figure 3.5. Spontaneous activity of extracellularly-recorded in vivo dopamine neuron ....... 35

Figure 3.6. First derivative-based spike detection of a pacemaker neuron ........................... 39

Figure 3.7. First derivative-based spike detection of a rebound bursting neuron ................. 40

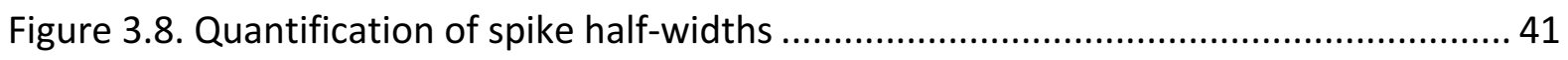

Figure 3.9. Quantification of sag amplitude, rebound delay, and input resistance ............... 41

Figure 3.10. Modality detection by density derivative estimation....................................... 43

Figure 4.1. Establishing the in vivo patch-clamp method for midbrain dopamine neurons .. 46 Figure 4.2. Maps illustrating the anatomical locations of 91 neurons recorded and identified as midbrain dopamine neurons with post hoc immunohistochemistry ............................. 48 Figure 4.3. Comparisons with cell-attached and extracellular recordings demonstrate the viability of whole-cell recordings

Figure 4.4. Plots of firing activity over time reveal stabilization of activity 50 seconds after

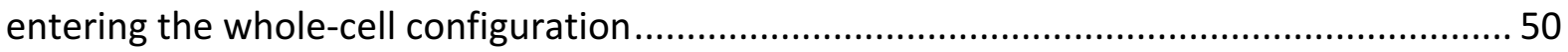

Figure 4.5. General electrophysiological properties of midbrain dopamine neurons ........... 52

Figure 4.6. In vivo dopamine neurons exhibit mixed firing patterns................................. 53

Figure 4.7. Dopamine neurons display a range of subthreshold membrane activity upon

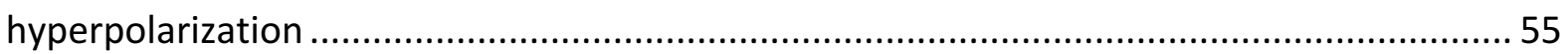

Figure 4.8. Dopamine neurons with unimodal $\mathrm{V}_{\min }$ and $\mathrm{V}_{\mathrm{thr}}$ distributions display pacemaker firing 
Figure 4.9. Dopamine neurons with bimodal $V_{\min }$ and unimodal $V_{\text {thr }}$ distributions show rebound burst firing

Figure 4.10. Dopamine neurons with multimodal $\mathrm{V}_{\min }$ and bimodal $\mathrm{V}_{\mathrm{thr}}$ distributions display plateau bursting

Figure 4.11. Pacemaker pattern of in vivo dopamine neurons exhibits great stability in subthreshold membrane behavior.

Figure 4.12. Rebound bursting of dopamine neurons exhibits hyperpolarized membrane potentials that signal the start of burst.

Figure 4.13. Plateau bursting of dopamine neurons shows plateau membrane potentials where burst terminates

Figure 4.14. Dopamine neurons can exhibit a combination of rebound and plateau bursting.

Figure 4.15. Properties of distinct subthreshold-based firing patterns identified by KDE derivative 75

Figure 4.16. Distribution of subthreshold membrane behavior-based firing patterns 78

Figure 5.1. Subthreshold membrane activity of dopamine neurons during the day versus night 80

Figure 5.2. Combining in vivo patch-clamp method with retrograde tracing 83

Figure 5.3. General electrophysiological properties of dopamine neurons projecting to nucleus accumbens lateral shell and medial shell 


\section{LIST OF TABLES}

Table 3.1. Program parameters for the DMZ Universal Electrode Puller............................. 32

Table 3.2. Summary of solutions used for experiments and their compositions................... 34

Table 3.3. List of primary antibodies used for immunohistochemistry............................... 37

Table 3.4. List of secondary antibodies and conjugates used for immunohistochemistry .... 37

Table 3.5. Number of $\mathrm{V}_{\min }$ and $\mathrm{V}_{\text {thr }}$ modes by subthreshold membrane activity-based firing pattern

Table 4.1. Summary of median(IQR) values for each class of subthreshold membrane activity-based firing patterns 76 


\section{LIST OF ABBREVIATIONS}

AADC

ACSF

ADP

AHP

AIS

ALDH1a1

AMPAR

AP

ATP

$\mathrm{BC}$

BG

$\mathrm{Ca}^{2+}$

$\mathrm{Cav}$

$\mathrm{CaCl}_{2}$

CB

CBGTC

ChR

$\mathrm{Cl}^{-}$

CLi

COMT

CS

CV

DA

DAT

DC

DDC

DLS

DMS

DOPA

DV
Aromatic L-amino acid decarboxylase

Artificial cerebrospinal fluid

Adenosine diphosphate

Afterhyperpolarization

Axon initial segment

Aldehyde dehydrogenase 1a1

a-Amino-3-hydroxy-5-methyl-4-isoxazolepropionic acid receptor

Anterior-posterior

Adenosine triphosphate

Bimodality coefficient

Basal ganglia

Calcium (ion)

Voltage-gated calcium channel

Calcium chloride

Calbindin

Cortico-basal ganglia-thalamo-cortical

Channelrhodopsin

Chloride (ion)

Caudal linear nucleus

Catechol-O-methyltransferase

Conditioned stimulus

Coefficient of variation

Dopamine

Dopamine transporter

Dark cycle

DOPA decarboxylase

Dorsolateral striatum

Dorsomedial striatum

I-3,4-dihydroxyphenylalanine

Dorsal-ventral 


\begin{tabular}{|c|c|}
\hline D1R & Dopamine receptor $D_{1}$ \\
\hline $\mathrm{D} 2 \mathrm{~L}$ & Dopamine receptor $D_{2}$ long isoform \\
\hline $\mathrm{D} 2 \mathrm{R}$ & Dopamine receptor $D_{2}$ \\
\hline D2S & Dopamine receptor $D_{2}$ short isoform \\
\hline EGTA & Ethylene glycol-bis( $\beta$-aminoethyl ether)-N, $N^{\prime} N^{\prime}, N^{\prime}$-tetraacetic acid \\
\hline$E / I$ & Excitatory-inhibitory \\
\hline FG & Fluoro-Gold \\
\hline FR & Firing rate \\
\hline GABA & $\mathrm{p}$-Aminobutyric acid \\
\hline GAD & Glutamate decarboxylase \\
\hline GAT & GABA transporter \\
\hline GIRK & G protein-coupled inwardly-rectifying potassium (channel) \\
\hline $\mathrm{GPe}$ & Globus pallidus externa; globus pallidus lateralis \\
\hline GPi & Globus pallidus interna; globus pallidus medialis \\
\hline GTP & Guanosine triphosphate \\
\hline $\mathrm{HCN}$ & Hyperpolarization-activated cyclic nucleotide-gated (channel) \\
\hline HDS & Hartigan's dip statistics \\
\hline HEPES & 4-(2-hydroxyethyl)-1-piperazineethanesulfonic acid \\
\hline HpTx3 & Heteropodatoxin-3 \\
\hline HVA & High-voltage activated \\
\hline HVA & Homovanillic acid \\
\hline $\mathrm{I}_{\mathrm{A}}$ & A-type potassium current \\
\hline $\mathrm{I}_{\mathrm{H}}$ & HCN current \\
\hline IF & Interfascicular nucleus \\
\hline IQR & Interquartile range \\
\hline ISI & Interspike interval \\
\hline $\mathrm{KCl}$ & Potassium chloride \\
\hline KGlu & Potassium gluconate \\
\hline $\mathrm{K}^{+}$ & Potassium (ion) \\
\hline $\mathrm{K}_{\mathrm{ir}}$ & Inward-rectifier potassium channel \\
\hline $\mathrm{K}_{\mathrm{v}}$ & Voltage-gated potassium channel \\
\hline KDE & Kernel density estimation \\
\hline
\end{tabular}




\begin{tabular}{|c|c|}
\hline KO & Knock-out \\
\hline K-ATP & ATP-sensitive potassium (channel) \\
\hline LC & Light cycle \\
\hline LLH & Lateral hypothalamus \\
\hline INAcC & Nucleus accumbens lateral shell \\
\hline LSCM & Laser scanning confocal microscope \\
\hline ISNc & Lateral substantia nigra pars compacta \\
\hline LVA & Low-voltage activated \\
\hline L-DOPA & levodopa; I-3,4-dihydroxyphenylalanine \\
\hline MAO & Monoamine oxidase \\
\hline $\mathrm{Mg}^{2+}$ & Magnesium (ion) \\
\hline $\mathrm{MgCl}_{2}$ & Magnesium chloride \\
\hline ML & Medial-lateral \\
\hline mNAcc & Nucleus accumbens medial shell \\
\hline mPFC & Medial prefrontal cortex \\
\hline $\mathrm{mSNc}$ & Medial substantia nigra pars compacta \\
\hline MSN & Medium spiny neuron \\
\hline $\mathrm{Na}^{+}$ & Sodium (ion) \\
\hline NAcc & Nucleus accumbens \\
\hline $\mathrm{Na}_{v}$ & Votage-gated sodium channel \\
\hline $\mathrm{Na}^{+} / \mathrm{K}^{+}$-ATPase & Sodium-potassium adenosine triphosphatase \\
\hline NB & Neurobiotin \\
\hline NET & Norepinephrine transporter \\
\hline $\mathrm{Ni}^{2+}$ & Nickel (ion) \\
\hline NMDA & $N$-Methyl-d-aspartic acid \\
\hline NMDAR & NMDA receptor \\
\hline PAG & Periaqueductal grey \\
\hline PBP & Parabrachial pigmented area \\
\hline PBS & Phosphate-buffered saline \\
\hline PFA & Paraformaldehyde \\
\hline PFC & Prefrontal cortex \\
\hline PN & Paranigral nucleus \\
\hline
\end{tabular}


PPTg Pedonculopontine tegmental nucleus

$\mathrm{RLi}$

Rostral linear nucleus of the raphe

RMTg

Rostromedial tegmental nucleus

RPE

Reward prediction error

RT

Room temperature

SD

Standard deviation

SK

Small conductance calcium-activated potassium (channel)

SNc

Substantia nigra pars compacta

$\mathrm{SNr}$

Substantia nigra pars reticulata

SPN

Spiny projection neuron

STN

Subthalamic nucleus

Str

Striatum

TD

Temporal difference

$\mathrm{TH}$

Tyrosine hydroxylase

TTX

Tetrodotoxin

tVTA

Tail of ventral tegmental area

US

Unconditioned stimulus

VGAT

Vesicular GABA transporter

VGLUT

Vesicular glutamate transporter

VMAT

Vesicular monoamine transporter

VMS

Ventromedial striatum

VP

Ventral pallidum

VTA

Ventral tegmental area

$\mathrm{V}_{\min }$

Minimum membrane voltage

$\mathrm{V}_{\text {thr }}$

Threshold membrane voltage 


\section{ABSTRACT}

Dopamine is a key neurotransmitter that serves several essential functions in daily behaviors such as locomotion, motivation, stimulus coding, and learning. Disrupted dopamine circuits can result in altered functions of these behaviors which can lead to motor and psychiatric symptoms and diseases. In the central nervous system, dopamine is primarily released by dopamine neurons located in the substantia nigra pars compacta (SNc) and ventral tegmental area (VTA) within the midbrain, where they signal behaviorally-relevant information to downstream structures by altering their firing patterns. Their "pacemaker" firing maintains baseline dopamine levels at projection sites, whereas phasic "burst" firing transiently elevates dopamine concentrations. Firing activity of dopamine neurons projecting to different brain regions controls the activation of distinct dopamine pathways and circuits. Therefore, characterization of how distinct firing patterns are generated in dopamine neuron populations will be necessary to further advance our understanding of dopamine circuits that encode environmental information and facilitate a behavior.

However, there is currently a large gap in the knowledge of biophysical mechanisms of phasic firing in dopamine neurons, as spontaneous burst firing is only observed in the intact brain, where access to intrinsic neuronal activity remains a challenge. So far, a series of highly-influential studies published in the 1980s by Grace and Bunney is the only available source of information on the intrinsic activity of midbrain dopamine neurons in vivo, in which sharp electrodes were used to penetrate dopamine neurons to record their intracellular activity. A novel approach is thus needed to fill in the gap. In vivo whole-cell patch-clamp method is a tool that enables access to a neuron's intrinsic activity and subthreshold membrane potential dynamics in the intact brain. It has been used to record from neurons in superficial brain regions such as the cortex and hippocampus, and more recently in deeper regions such as the amygdala and brainstem, but has not yet been performed on midbrain dopamine neurons. Thus, the deep brain in vivo patch-clamp recording method was established in the lab in an attempt to investigate the subthreshold membrane potential dynamics of tonic and phasic firing in dopamine neurons in vivo. 
The use of this method allowed the first in-depth examination of burst firing and its subthreshold membrane potential activity of in vivo midbrain dopamine neurons, which illuminated that firing activity and subthreshold membrane activity of dopamine neurons are very closely related. Furthermore, systematic characterization of subthreshold membrane patterns revealed that tonic and phasic firing patterns of in vivo dopamine neurons can be classified based on three distinct subthreshold membrane signatures: 1) tonic firing, characterized by stable, non-fluctuating subthreshold membrane potentials; 2) rebound bursting, characterized by prominent hyperpolarizations that initiate bursting; and 3) plateau bursting, characterized by transient, depolarized plateaus on which bursting terminates. The results thus demonstrated that different types of phasic firing are driven by distinct patterns of subthreshold membrane activity, which may potentially signal distinct types of information. Taken together, the deep brain in vivo patch-clamp technique can be used for the investigation of firing mechanisms of dopamine neurons in the intact brain and will help address open questions in the dopamine field, particularly regarding the biophysical mechanisms of burst firing in dopamine neurons that control behavior. 


\section{GERMAN SUMMARY}

Dopamin ist ein essenzieller Neurotransmitter, der mehrere wesentliche Funktionen bei alltäglichen Verhaltensweisen wie Fortbewegung, Motivation, Reizkodierung und Lernen erfüllt. Störungen im dopaminergen System können zu veränderten Funktionen dieser Verhaltensweisen führen, was $\mathrm{zu}$ motorischen und psychiatrischen Symptomen und Krankheiten führen kann. Im zentralen Nervensystem wird der Großteil des Dopamins von dopaminergen Neuronen freigesetzt, die sich in der Substantia nigra pars compacta (SNc) und im ventralen tegmentum (VTA) im Mittelhirn befinden, wo sie verhaltensrelevante Informationen an nachgeschaltete Strukturen überitteln, indem sie ihre Feuerungsmuster verändern. Ihre "Schrittmacher"-Aktivität hält die Dopamin-Konzentration an den Projektionsstellen auf einem konstant niedrigen Niveau aufrecht, während phasisches "Burst"-Feuern die Dopamin-Konzentration vorübergehend ansteigen lässt.

Dopaminerge Neurone im Mittelhirn innervieren viele Hirnregionen; insbesondere stellen sie besonders dichte Verbindungen mit GABAergen dornentragenden Projektionsneuronen (SPNs) im Striatum her. Das Striatum ist ein wichtiger Bestandteil einer Gruppe von subkortikalen Kernen, die Basalganglien (BG) genannt werden und die motorisches und limbisches Verhalten steuern. Als Eingangszentrum der BG empfängt das Striatum Eingänge aus SNc und VTA, sowie vom Kortex und Thalamus und sie leitet Informationen über direkte und indirekte Bahnen durch die Ausgangskerne der BG zurück an den Thalamus. Der Schlüsselfaktor zur Unterscheidung von direkten und indirekten BG-Bahnen ist die Dopaminrezeptorexpression in den SPNs, deren Natur über die weitere Bearbeitung des Dopaminsignals bestimmt. SPNs des direkten Signalweges exprimieren vorwiegend den Dopaminrezeptor $\mathrm{D}_{1}$ (D1R), der bei Dopaminbindung zu einer Erregung der SPNs führt, wohingegen der Dopaminrezeptor $\mathrm{D}_{2}$ (D2R) vorwiegend in SPNs des indirekten Signalweges exprimiert wird, der eine hemmende Wirkung auf SPNs hat. D1R und D2R unterscheiden sich in ihrer Affinität zum Dopamin: der exzitatorische D1R hat eine geringere Affinität zum Dopamin, während der hemmende D2R eine höhere Affinität aufweist. Tonisches Feuern von dopaminergen Neuronen hält somit das Gleichgewicht 
zwischen des direkten und indirekten Signalweges aufrecht, während phasisches Feuern den Dopaminspiegel transient erhöht, was zu einer verstärkten Aktivierung der D1RBahn führt. Folglich steuert die Feuerungsaktivität der dopaminergen Neuronen, die in unterschiedliche Hirnregionen projizieren, die Aktivierung verschiedener dopaminerger Signalwege. Daher ist eine Charakterisierung der Art und Weise, wie unterschiedliche Feuerungsmuster in dopaminergen Neuronen erzeugt werden, notwendig, um unser Verständnis der Dopamin-Schaltkreise und ihre Rolle bei der Verarbeitung von Umweltinformationen und die Steuerung des Verhaltens weiter zu verbessern.

Allerdings gibt es derzeit eine große Wissenslücke bezüglich der biophysikalischen Mechanismen des phasischen Feuerns in dopaminergen Neuronen, da spontane Burst-Aktivität nur im intakten Gehirn beobachtet wird. Bislang ist eine Reihe von sehr einflussreichen Studien, die in den 1980er Jahren von Grace und Bunney veröffentlicht wurden, die einzige Quelle für Informationen über die intrinsische Aktivität dopaminerger Neurone im Mittelhirn in vivo, in denen feine Glaselektroden verwendet wurden, um in dopaminerge Neuronen einzudringen und ihre intrazelluläre Aktivität aufzuzeichnen. Daher ist ein neuer Ansatz erforderlich, um diese Wissenslücke zu schließen. Die in vivo Ganzzell-Patch-Clamp-Methode ist eine Methode, das den elektrischen Zugang zur intrinsischen Aktivität eines Neurons und ihrer unterschwelligen Membranpotentialdynamik im intakten Gehirn ermöglicht. Sie wurde zur Aufzeichnung von Neuronen in oberflächlichen Hirnregionen wie dem Kortex und dem Hippocampus und in jüngerer Zeit auch in tieferen Regionen wie der Amygdala und des Hirnstammes verwendet, jedoch nicht an dopaminergen Neuronen des Mittelhirns. Daher wurde in unserem Labor die in vivo Patch-Clamp-Methode für dopaminerge Neurone etabliert, um die unterschwellige Membranpotenzialdynamik des tonischen und phasischen Feuerns dopaminerger Neurone in vivo zu untersuchen.

Mit Hilfe der blinden Patch-Methode, begleitet durch ein Tongerät für auditorisches Feedback, konnte ich 141 mutmaßliche dopaminerge Neuronen in vivo aufzeichnen, von denen 91 immunhistochemisch als dopaminerge Neuronen im Mittelhirn bestätigt wurden. Um die Qualität meiner Aufzeichnungen zu bestätigen, wurde die spontane Aktivität dopaminerger Neuronen aus on-cell Aufzeichnungen mit der Aktivität extrazellulärer Messungen verglichen, was zeigte, dass die On-cell-Aktivität 
dopaminerger Neurone in vivo mit der Aktivität unperturbierter extrazellulärer Messungen vergleichbar war. Die Untersuchung der Feuerungsaktivität, die im wholecell Modus über die Zeit aufgezeichnet wurde, zeigte, dass die Feuerungs-Aktvität nicht unmittelbar nach derAufbrechen der Membran stabil war, sondern sich ca. nach 50 Sekunden stabilisierte. Ein Vergleich der On-Cell- mit der stabilen Whole-Cell-Aktivität (z. B. 50 Sekunden nach Aufbrechen der Membran) zeigte, dass dopaminerge Neurone ihr Feuerungsmuster in der Whole-Cell-Konfiguration beibehalten konnten.

In-vitro-Studien an dopaminergen Neuronen haben gezeigt, dass die Mehrheit dieser Neurone eine "Sag"-Komponente aufweist, d. h. einen ausgeprägten Einwärtsstrom, der durch den Hyperpolarisation-aktivierten-zyklischen-Nukleotidgesteuerten (HCN) Kanal als Reaktion auf eine Hyperpolarisation vermittelt wird. Darüber hinaus gibt es auch Subpopulationen dopaminerger Neurone, die keinen „Sag“Komponente aufweisen, dafür aber einen langen Rebound zeigen, welches die Latenz zum ersten Aktionspotential nach Ende der Hyperpolarisation darstellt. Um herauszufinden, ob dopaminerge Neurone in vivo in ähnlicher Weise auf eine Hyperpolarisation reagieren, habe ich Strominjektionsprotokolle für eine einige der gemessenen Neuronen durchgeführt. Wie aus der Literatur zu erwarten war, zeigten die meisten Neurone bei hyperpolarisierten Membranpotentialen eine „Sag“-Komponente, während ihre Rebound-Aktivität im Vergleich zu in vitro beobachteten Aktivitäten vielfältiger waren und von keiner merklichen Reaktion, lange Rebounds bis hin zu BurstAktivitäten reichten. Darüber hinaus wurden für diese dopaminergen Neurone ein Eingangswiderstand von ca. $280 \mathrm{M} \Omega$ berechnet, was in starkem Kontrast zu den bekannten Werten von $31 \mathrm{M} \Omega$ für intrazellulär mit der feinen Glaselektroe gemessenen In-vivo-Dopamin-Neuronen stand, dafür näher am Eingangswiderstand von synaptisch isolierten In-vitro-Dopamin-Neuronen von ca. $450 \mathrm{M} \Omega$ lag. Der relativ hohe Eingangswiderstand würde darauf hindeuten, dass dopaminerge Neurone im Vergleich zu anderen Nervenzellen resistenter gegen Shunting-Phänomene sind als bisher angenommen.

Die initiale Untersuchung der rohen Messdaten führte zu der Hypothese, dass die spontane Feuerungsaktivität dopaminerger Neurone im Mittelhirn in vivo eng mit den Fluktuationen der unterschwelligen Membranpotentiale zusammenhängt. Darüber 
hinaus folgten auf die hyperpolarisierende Membranpotentiale, die in der Regel längere ISIs bedingten, häufig hochfrequente Aktionspotentiale. Die subschwellige Membranaktivität könnte potenziell genügend Informationen beinhalten, um unterschiedliche tonische und phasische Feuerungsmuster dopaminerger Neurone in vivo zu identifizieren, die von dieser subschwelligen Aktivität abhängig sind. Um die subschwellige Membranaktivität dopaminerger Neurone in vivo während der Spontanaktivität $\mathrm{zu}$ charakterisieren, zeichnete ich die Histogramme der ISIMembranpotentialminima $\left(\mathrm{V}_{\min }\right)$ und zusätzlich die Histogramme der Aktionspotentialschwellen ( $\mathrm{V}_{\text {thr }}$ ) auf, um zu sehen, ob $\mathrm{V}_{\text {thr }}$ auch zusammen mit $\mathrm{V}_{\text {min }}$ fluktuiert. Wie erwartet zeigten die Histogramme von $V_{\min }$ und $V_{\text {thr }}$ bei einigen dopaminergen Neuronen klare unimodale oder bimodale Verteilungen, aber interessanterweise wiesen $V_{\text {min }}$ und $V_{\text {thr }}$ nicht immer identische Verteilungsmuster auf. Tatsächlich schien eine multimodale Verteilung von $V_{\text {thr }}$ einer anderen Art von BurstAktivität zu unterliegen als die von $V_{\min }$. Basierend auf diesen Befunden identifizierte ich drei verschiedene Typen von in vivo subschwelligen Membransignaturen in dopaminergen Neuronen, die die Grundlage für deren Feuerungsmuster bilden: 1) unimodale $V_{\text {min- }}$ und $V_{\text {thr }}$-Verteilungen, 2) eine bimodale $V_{\min }$-Verteilung und eine unimodale $V_{\text {thr }}$-Verteilung, und 3 ) eine bimodale/multimodale $V_{\min }$-Verteilung und eine bimodale $V_{\text {thr }}$-Verteilung.

Die weitere Charakterisierung dopaminerger Neurone, die zu jeder der drei Klassen gehörten, ergab, dass sie der unterschiedlichen Feuerungsaktivität dopaminerger Neuronen entsprachen: unimodale Verteilungen wurden mit tonischem Feuern assoziiert, während bimodale Verteilungen mit verschiedenen Arten von phasischem Feuern verbunden waren. Darüber hinaus war die bimodale $V_{\min }$-Verteilung ein Merkmal des Rebound-Bursting, bei dem der Burst-Aktivität eine ausgeprägte

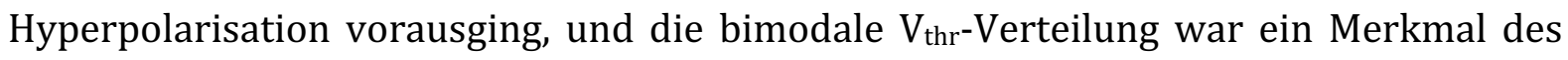
Plateau-Bursting, bei dem die Burst-Aktivität am oberen Ende der transienten PlateauMembranpotentiale auftrat. Das Auftragen von ISI gegen $V_{\min }$ und $V_{\text {thr }}$ führte zu klar getrennten Clustern, wenn die $\mathrm{V}_{\text {min- }}$ oder $\mathrm{V}_{\text {thr }}$-Verteilung bimodal war. Eine weitere Analyse von Spikes, die mit $\mathrm{V}_{\mathrm{min}}$ - oder $\mathrm{V}_{\text {thr-Clustern }}$ assoziiert waren, und deren zwei benachbarten Spikes als Drei-Spike-Sequenzen ergab, dass hyperpolarisiertes $V_{\min }$ den 
Beginn des Bursts signalisierte und dass das nachfolgende Aktionspotential (erster Spike des Bursts) eine stärker hyperpolarisierte Spike-Schwelle und eine erhöhte Depolarisationsrate aufwies, wohingegen depolarisiertes $V_{\text {thr }}$ die Beendigung des Bursts signalisierte und dass diese Verschiebung in $\mathrm{V}_{\text {thr }}$ von einer verringerten Depolarisationsrate begleitet wurde. Die beiden Arten des phasischen Feuerns, Reboundund Plateau-Bursting, steuern also die Initiierung bzw. Beendigung des Burst-Feuerns, was darauf hindeutet, dass sie unterschiedliche Arten von Informationen kodieren können.

Insgesamt ermöglichte die in-vivo-Patch-Clamp-Methode für tiefe Hirnregionen die erste systematische Analyse der unterschwelligen Membrandynamik dopaminerger Neurone in vivo, die zeigte, dass die unterschwellige Membranaktivität das Feuern in dopaminergen Neuronen antreibt und dass drei verschiedene Arten von Aktivitätsmustern basierend auf den unterschwelligen Membransignaturen identifiziert werden können. Die Methode kann somit für die Untersuchung der Feuermechanismen dopaminerger Neurone im intakten Gehirn verwendet werden und könnte dazu beitragen, offene Fragen im Dopamin-Forschungsbereich zu klären, insbesondere im Hinblick auf die biophysikalischen Mechanismen der Burst-Aktivität dopaminerger Neurone, die das Verhalten steuern. 


\section{INTRODUCTION}

\subsection{Dopaminergic systems in the brain}

Just weighing approximately $1.5 \mathrm{~kg}$ and with estimated 86 billion neurons, our brain is responsible for everything that we do: from eating to running; from learning to sleeping. It processes sensory information and integrates inputs from multiple sources, while simultaneously processing cognition and coordinating motor action (Azevedo et al., 2009; Kandel, 2013). Anatomically, the mammalian brain can be divided into six anatomical regions: the telencephalon, diencephalon, mecencephalon (midbrain), cerebellum, pons, and medulla oblongata. Some of these regions can be further divided into anatomically and functionally distinct subregions, such as the cerebral cortex and hippocampus which are part of the telencephalon, and the thalamus and hypothalamus which make up the diencephalon (Kandel, 2013).

Two such subregions located in the midbrain, the substantia nigra pars compacta (SNc) and the ventral tegmental area (VTA), are the main sources of dopamine in the brain. Dopamine (3,4-dihydroxyphenethylamine, DA) is a monoamine neurotransmitter belonging to the catecholamine and phenylalanine families and plays important roles in salience, reward- and aversion-based learning, habit formation, as well as motor control (Berridge, 2007; Lammel et al., 2014; Schultz, 2019, 2007; Watabe-Uchida et al., 2017; Yin and Knowlton, 2006). Disruption of this system that leads to altered DA signaling is linked to disorders including Parkinson's disease, schizophrenia, and addiction (Grace, 2016; Howes et al., 2017; Kesby et al., 2018; Koob and Volkow, 2016; Maia and Frank, 2011; Volkow et al., 2009). DA's discovery came in 1910 when it was first synthesized by George Barger and James Ewens and described by Barger and Henry Dale in the same year (Barger and Dale, 1910; Hornykiewicz, 2002). Central DA research was subsequently pushed forward by two milestones that happened in the late 1950s: Kathleen Montagu's discovery of DA presence in the brain, and Arvid Carlsson's Nobel Prize-winning revelation which pinned DA as a central neurotransmitter (Benes, 2001; Carlsson et al., 1958, 1957; Montagu, 1957). The essential functions DA plays in the brain have since become increasingly evident through decades of studies, and new research 
continues to add to and modify our understanding of the DA system, in particular with emerging novel methods.

\subsubsection{Molecular pathways of the dopaminergic system}

DA homeostasis is maintained through several mechanisms such as synthesis, storage, release, diffusion, reuptake, and degradation. Biochemically, DA is synthesized from a precursor amino acid L-tyrosine, which is first converted into levodopa (L-DOPA) by the enzyme tyrosine hydroxylase (TH), and L-DOPA is subsequently converted into DA by aromatic L-amino acid decarboxylase (AADC), also known as DOPA decarboxylase (DDC) (Fig. 1.1). TH is the rate-limiting enzyme of DA biosynthesis, and the antibody against TH is widely used for immunocytochemistry which reliably detects DA neurons in the midbrain. TH is also found in other parts of the brain such as the locus coeruleus where epinephrine or norepinephrine is abundant, as DA itself is a precursor for these two catecholamine neurotransmitters (Mulvihill, 2019; Nestler et al., 2015). Once synthesized, DA is transported into synaptic vesicles via vesicular monoamine transporter 2 (VMAT2), where it is stored until release (Eiden et al., 2004). Evidence indicates that TH and AADC interact with VMAT2 at the synaptic vesicle membrane, suggesting that DA synthesis and transport may be coupled rather than occurring independently (Cartier et al., 2010). In addition, there are populations of VTA DA neurons that corelease the inhibitory neurotransmitter gamma-aminobutyric acid (GABA) or the excitatory neurotransmitter glutamate along with DA (Chuhma et al., 2017; Miranda-Barrientos et al., 2021; Morales and Margolis, 2017; Sulzer et al., 1998;

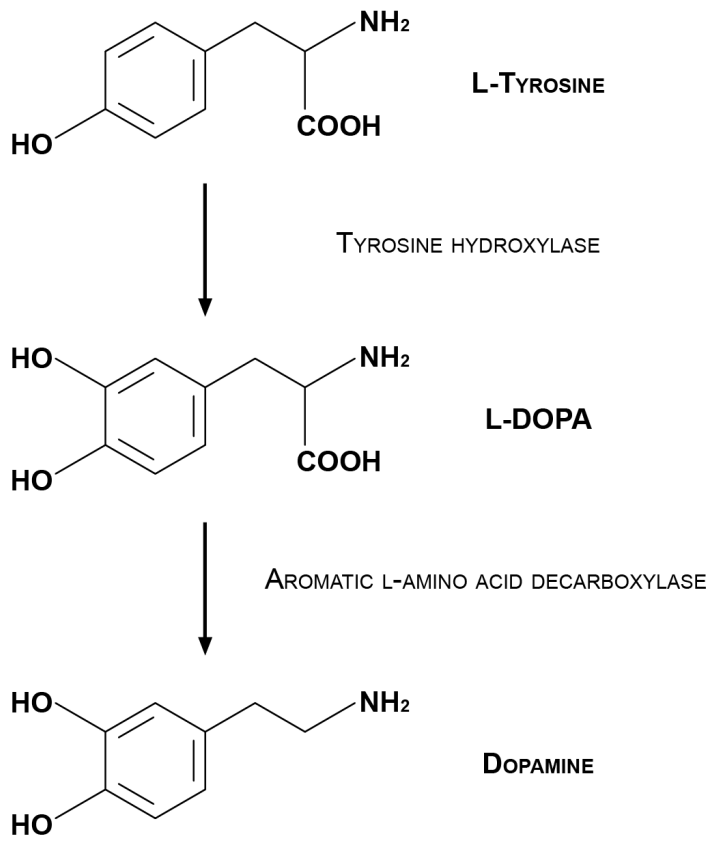

Figure 1.1. Biosynthesis of dopamine. During dopamine biosynthesis, amino acid L-tyrosine is converted to L-DOPA by the enzyme tyrosine hydroxylase, followed by the conversion of L-DOPA into dopamine by the enzyme aromatic amino acid decarboxylase. Based on Nestler et al., 2015. 
Tecuapetla et al., 2010; Tritsch et al., 2012; Ungless and Grace, 2012). While the canonical biosynthesis of GABA involves the enzyme glutamate decarboxylase (GAD), GABA that is co-released with DA may be either synthesized by the non-canonical aldehyde dehydrogenase 1a1 (ALDH1a1) or taken up from the extracellular space via GABA transporters 1 and 4 (GAT1 and GAT4). GABA is then transported into vesicles along with DA by VMAT2, rather than by vesicular GABA transporter (VGAT) (Kim et al., 2015; Tritsch et al., 2014, 2012). Unlike GABA, glutamate is not co-released from the same synaptic vesicles as DA: it is packaged into vesicles by vesicular glutamate transporter 2 (VGLUT2) and co-transmitted with DA but from a different microdomain and onto a different synapse (Dal Bo et al., 2004; Sulzer et al., 1998; Zhang et al., 2015). Once action potentials are evoked, DA is released into the extracellular space by exocytosis, where it binds to receptors with different levels of affinity to DA that are located at presynaptic,

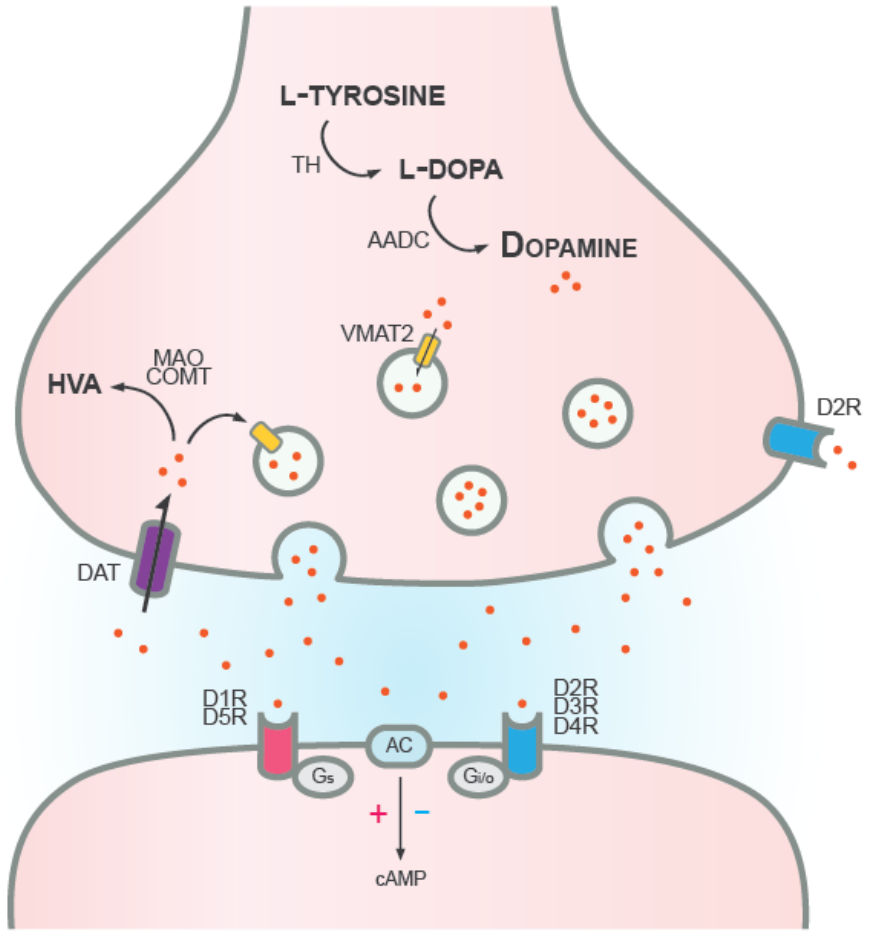

Figure 1.2. Model of dopamine synapse. A simplified overview of molecular pathways for dopamine synthesis, packaging, binding, and reuptake is illustrated. AADC: aromatic L-amino acid decarboxylase; AC: adenylyl cyclase; cAMP: cyclic adenosine monophosphate; COMT: catechol-O-methyltransferase; DAT: dopamine transporter; D1R-D5R: dopamine receptors D1-D5; HVA: homovanillic acid; MAO: monoamine oxidase; TH: tyrosine hydroxylase; VMAT2: vesicular monoamine transporter 2. Based on Borroto-Escuela et al., 2018; Nestler et al., 2015; Mulvihill, 2019 \& Tritsch and Sabatini, 2012. postsynaptic, and extrasynaptic sites. There are currently five known subtypes of DA receptors: DA receptors $D_{1}, D_{2}, D_{3}, D_{4}$, and $D_{5}$ (D1R-D5R), all of which are G protein-coupled receptors and are differentially expressed throughout the brain (Beaulieu and Gainetdinov, 2011; Missale et al., 1998). D1R and D2R are the most widely expressed subtypes of the five, and in the striatum where DA is most abundant, D1R appears to be in a low-affinity state, whereas D2R is in a highaffinity state (Cumming, 
2011; Dreyer et al., 2010; Jackson and Westlind-Danielsson, 1994; Richfield et al., 1989). Upon binding to DA, D1R and D5R, belonging to the $\mathrm{D}_{1}$-like family, activate the Gs alpha subunit, which activates adenylyl cyclase and increases cyclic adenosine monophosphate (cAMP), leading to increased protein kinase A activity. In contrast, D2R, D3R, and D4R from the $D_{2}$-like family are coupled to the $G_{i / o}$ alpha subunit, which inhibits adenylyl cyclase and decreases cAMP production, leading to inhibition of protein kinase A (see also Subsection 1.2.3) (Beaulieu and Gainetdinov, 2011; Missale et al., 1998). Thus, DA receptors from the $D_{1}$-like family have an excitatory effect whereas those from the $D_{2}$ like family exert an inhibitory effect. Furthermore, D2R, and likely also D3R, works as an autoreceptor to provide a negative feedback mechanism to control DA synthesis and release (Beaulieu and Gainetdinov, 2011; Ford, 2014; Missale et al., 1998). Alternative splicing results in two isoforms of D2R with distinct functions: D2R short form (D2S), expressed on presynaptic membranes, primarily functions as an autoreceptor; D2R long form (D2L), which is thought to mainly work as a postsynaptic receptor, may also be capable of autoinhibition, as indicated by recent evidence (Gantz et al., 2015; Jang et al., 2011; Usiello et al., 2000). All DA neurons except those projecting to the medial prefrontal cortex (mPFC) appear to express $\mathrm{D}_{2}$ autoreceptors and are inhibited by DA (Chiodo et al., 1984; Lacey et al., 1987; Lammel et al., 2008). Similar to other neuromodulators, extrasynaptic/somatodendritic volume transmission -rather than synaptic transmission- is thought to be the main mode of DA signaling (Borroto-Escuela et al., 2018; Fuxe et al., 2010). According to a computational model, these DA transients bind to high-affinity DA receptors within a 7-8 $\mu \mathrm{m}$ radius or to low-affinity DA receptors within $\sim 2 \mu \mathrm{m}$ from the release site (Rice and Cragg, 2008). Importantly, DA release occurs at axonal terminals but also at somatodendritic sites within the SNc and VTA to regulate their own firing patterns (Chen et al., 2011; Geffen et al., 1976; Groves and Linder, 1983; Rice and Patel, 2015). Remaining DA spillover is transported back into the presynaptic DA neuron via dopamine transporters (DAT) along with co-substrates, sodium $\left(\mathrm{Na}^{+}\right)$and chloride $\left(\mathrm{Cl}^{-}\right)$ions (McElvain and Schenk, 1992). In addition, the prefrontal cortex (PFC) has lower DAT expression as compared to other projection sites of DA neurons, and it appears that noradrenergic neurons mechanism in the PFC via norepinephrine transporters (NET) instead of DAT and with a much slower. Once back inside the cell, DA 
is either recycled through repackaging by VMAT2, or degraded by the enzymes monoamine oxidase (MAO) and catechol-O-methyltransferase (COMT) into homovanillic acid (HVA). Additionally, DA can be taken up by astrocytes for degradation by MAO and COMT, which appears to be a critical component of DA metabolism in the PFC (Käenmäki et al., 2010; Mulvihill, 2019; Nestler et al., 2015; Petrelli et al., 2020). A summary schematic is shown in Figure 1.2.

\subsubsection{Anatomy and connectivity of the dopaminergic system}

The substantia nigra -Latin for "black substance"-, was named due to its dark appearance caused by neuromelanin and consists of two components: pars compacta (SNc) and pars reticulata (SNr). The SNc primarily consists of DA neurons, whereas the SNr contains primarily GABAergic neurons, with a small percentage of GABAergic neurons in the SNc and vice versa (Björklund and Dunnett, 2007; Gantz et al., 2018). The VTA consists of several subregions, although there is yet to be a consensus on what structures comprise the VTA (Trutti et al., 2019). Nevertheless, researchers have suggested that the following five anatomical subdivisions be included in the VTA: the DArich nuclei parabrachial pigmented area (PBP) and paranigral nuclei (PN); and the caudal linear nucleus (CLi), interfascicular nucleus (IF), and rostral linear nucleus of the raphe (Rli) with little to no DA neurons (Halliday and Törk, 1986; Morales and Margolis, 2017; Trutti et al., 2019). The VTA is highly heterogeneous in comparison to the SNc and interspersed with approximately 60-70\% DAergic neurons, 30-40\% GABAergic neurons,

a small percentage of glutamatergic neurons, as well as neurons that co-release a combination of the three neurotransmitters (Bouarab et al., 2019; Miranda-Barrientos et al., 2021; Morales and Margolis, 2017; Olson and Nestler, 2007; Poulin et al., 2018; Sulzer et al., 1998; Tecuapetla et al., 2010; Tritsch et al., 2012; Ungless and Grace, 2012; Yamaguchi et al., 2011). GABAergic neurons in the VTA either locally regulate the activity of DA neurons or send long-range projections to cholinergic neurons in the striatum (Bouarab et al., 2019; Creed et al., 2014). While the SNc and VTA are technically considered two distinct regions, no clear boundary segregates the two (Björklund and Dunnett, 2007; Gantz et al., 2018). 
To describe DA neuron populations, a cell group nomenclature system originally proposed by Dahlström and Fuxe in 1964 is still in use today that classifies groups of mammalian neurons that contain catecholamines (Dahlstroem and Fuxe, 1964). The currently accepted DAergic cell groups, A8 to A17, are mainly situated within the subcortical structures, with the most dense areas being the SNc (A9), VTA (A10), and reticular formation or retrorubral field in mice (A8), and the less dense areas being the diencephalon (A11-A15), olfactory bulb (A16), and retina (A17) (Björklund and Dunnett, 2007; Dahlstroem and Fuxe, 1964; Hökfelt et al., 1984a, 1984b; Kosaka et al., 1987; Manger et al., 2002; Smith and Kieval, 2000). The total number of DA neurons (e.g. tyrosine hydroxylase immunopositive) residing in A8-A10 is estimated to be 20,00030,000 for mice, $160,000-320,000$ for monkeys, and 400,000-600,000 for humans (Björklund and Dunnett, 2007; German and Manaye, 1993; Nelson et al., 1996). Out of the four major DA pathways, three originate in the SNc (nigrostriatal pathway) and VTA (mesocortical and mesolimbic pathways), and one originates in the arcuate nucleus of the hypothalamus (A12) and projects to the pituitary gland (tuberoinfundibular pathway). The SNc and VTA belong to a group of subcortical nuclei called the basal ganglia (BG) and related structures, which consist of several nuclei located in the diencephalon as well as in the midbrain: the striatum (dorsally, caudate and putamen; ventrally,

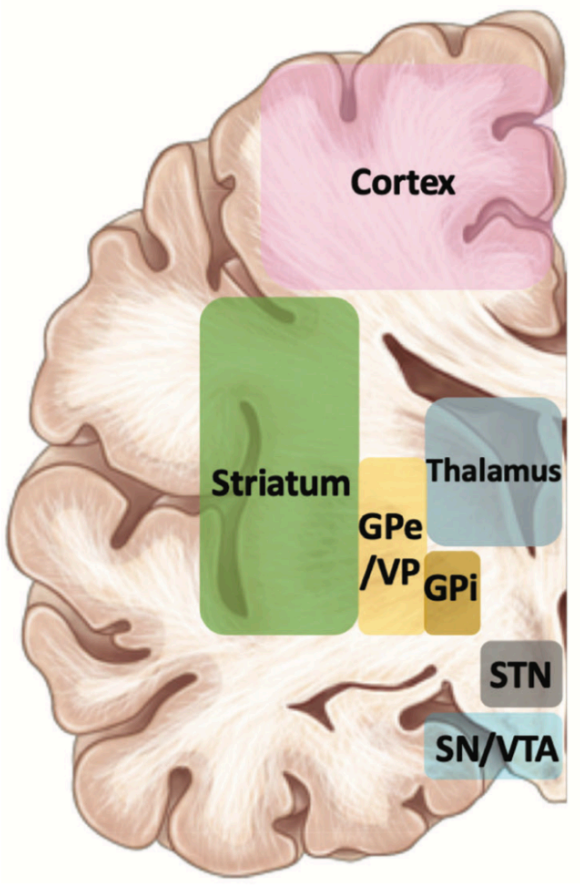

Figure 1.3. Brain regions involved in the cortico-basal ganglia-thalamo-cortical loop. The striatum, globus pullidus externa, ventral pallidum, globus pallidus interna, subthalamic nucleus, substantia nigra pars compacta and ventral tegmental area form the basal ganglia (and related structures). Information from the cortex and thalamus is fed to the basal ganglia and relayed back to the thalamus and then to the cortex. Anterior-posterior plane is disregarded in this schematic. GPe: globus pallidus externa; GPi: globus pallidus interna; SN: substantia nigra; STN: subthalamic nucleus; VP: ventral pallidum; VTA: ventral tegmental area. From "Role of basal ganglia neurocircuitry in the pathology of psychiatric disorders," by T. Macpherson and T. Hikida, 2019, Psychiatry and Clinical Neurosciences, p.290. Copyright (2019) John Wiley and Sons. Reprinted with permission. 
nucleus accumbens and olfactory tubercle), globus pallidus interna and globus pallidus externa (in rodents, entopeduncular nucleus and globus pallidus, respectively), ventral pallidum, substantia nigra pars reticulata and substanitia nigra pars compacta, and subthalamic nucleus (Fig. 1.3) (Grillner and Robertson, 2016; Gunaydin and Kreitzer, 2016; Humphries and Prescott, 2010; Ikemoto, 2007; Lanciego et al., 2012). Midbrain DA neurons receive inputs from many brain regions, but notably the cortex, striatum, lateral

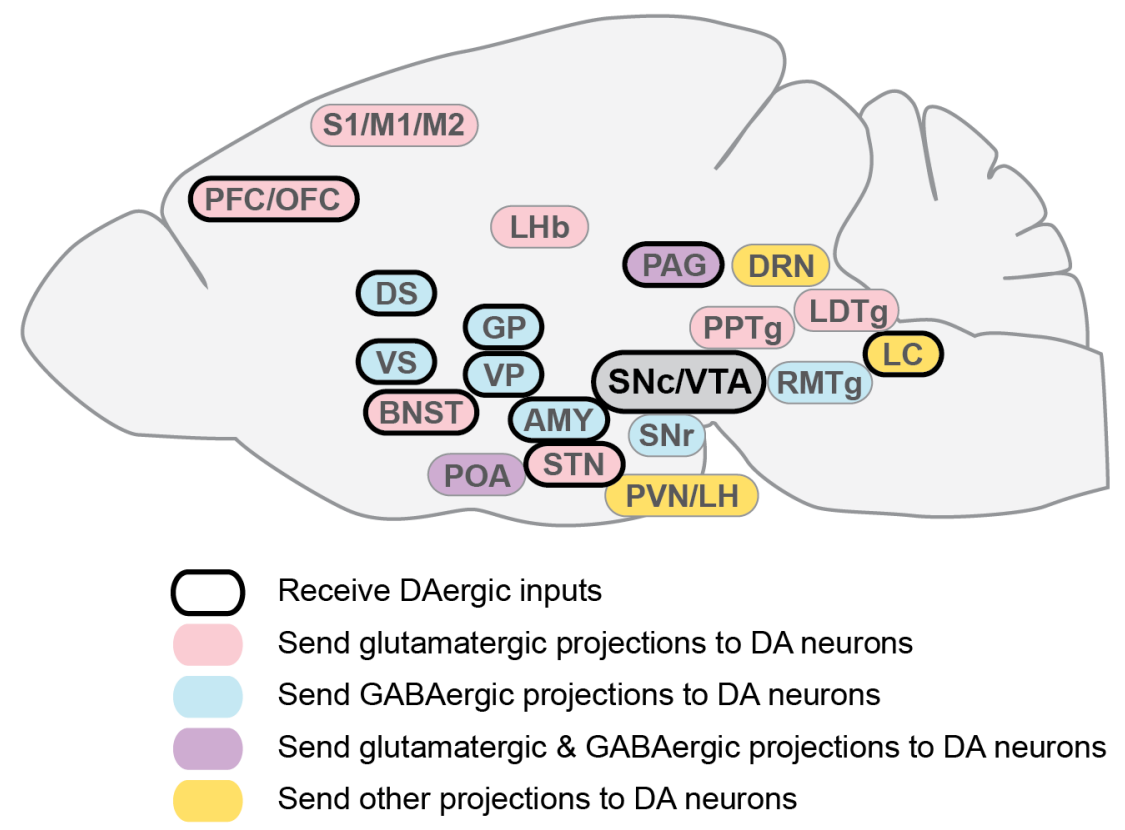

Figure 1.4. Inputs and outputs of dopamine neurons. Dopamine neurons in the SNc and VTA receive projections from numerous brain regions. PFC, LHb, PPTg, LDTg, and BNST send glutamatergic inputs to dopamine neurons, while DS, NAcc, GP, VP, AMY, SNr, and RMTg send GABAergic projections. PAG and POA send both glutamatergic and GABAergic afferents. In addition, dopamine neurons also receive neuromodulatory inputs: glutamate and serotonin from DRN, norepinephrine from LC, orexin from LH, and oxytocin from PVN. Some of these nuclei make reciprocal connections with dopamine neurons. Dopamine neurons' output nuclei are PFC/OFC, DS, VS, GP, VP, BNST, AMY, PAG, LC and STN. AMY: amygdala; BNST: bed nucleus of stria terminalis; DRN: dorsal raphe nucleus; DS: dorsal striatum; GP: globus pallidus; LC: locus coeruleus; LDTg: laterodorsal tegmental nucleus; LH: lateral hypothalamus; LHb: lateral habenula; M1: primary motor cortex; M2: secondary motor cortex; OFC: orbitofrontal cortex; PAG: periaquedactal gray; PFC: prefrontal cortex; POA: preoptic area; PPTg: pedunculopontine tegmental nucleus; PVN: paraventicular nucleus of hypothalamus; RMTg: rostromedial tegmental nucleus; S1: primary somatosensory cortex; SNc substantia nigra pars compacta; SNr: substantia nigra pars reticulata; STN: subthalamic nucleus; VP: ventral pallidum; VS: ventral striatum; VTA: ventral tegmental area. The list is not exhaustive. Based on Beier et al., 2015; Fang et al., 2018; Lanciego et al., 2012; Morales and Margolis, 2017; Ntamati et al., 2018; Park et al., 2017; Stamatakis et al., 2014; Steinberg et al., 2020; Tyree and de Lecea, 2017; Wang et al., 2019; Watabe-Uchida et al., 2017 \& Xiao et al., 2017. 
hypothalamus (LH), rostromedial tegmental nucleus (RMTg; also called the tail of VTA, tVTA), dorsal raphe nucleus (DRN), as well as periaqueductal grey (PAG) (Fig. 1.4) (Beier et al., 2015; Morales and Margolis, 2017; Watabe-Uchida et al., 2017). In turn, axons of DA neurons innervate the cortex, amygdala (AMY), and PAG, and make particularly dense connections within the striatum (Gerfen, 2000; Morales and Margolis, 2017; Ntamati et al., 2018). The striatum, which was initially described in an English physician Thomas Willis' book Cerebri anatome (1664) as the corpus striatum, is strongly interconnected with several loops and is part of the BG circuit essential for motor control, but also habit formation, reinforcement learning, and decision-making (Grillner and Robertson, 2016; Hikosaka et al., 2018; Lanciego et al., 2012; Macpherson et al., 2014; Mink, 1996; Parent, 2012; Yin and Knowlton, 2006). Unsurprisingly, dysfunction of these BG circuits is implicated in movement disorders such as Parkinson's disease, Huntington's disease, dystonia (Albin, 1995; Mink, 2003; Wichmann and DeLong, 1996), as well as in psychiatric conditions (Grace, 2016; Gunaydin and Kreitzer, 2016; Howes et al., 2017; Kesby et al., 2018; Maia and Frank, 2011).

The 'classical' model of the BG function was initially described as having two dichotomous, functionally-opposing circuits: the direct pathway facilitating voluntary movement, and the indirect pathway inhibiting movement (Albin et al., 1989; Bolam et al., 2000; Parent and Hazrati, 1995). The key factor differentiating these two pathways is the DA receptor expression in the striatal spiny projection neurons (SPNs), also known as medium spiny neurons (MSN). SPNs in the direct pathway predominantly express D1R, which leads to SPN excitation, whereas the indirect pathway SPNs predominantly express D2R, which results in SPN inhibition. DA thus bears a dual effect on the BG circuit through $\mathrm{D}_{1}$ and $\mathrm{D}_{2}$ signaling to control the BG output (Cazorla et al., 2014; Gerfen et al., 1990). SPNs represent approximately 90 to $95 \%$ of the neuronal population in the striatum and serve as the input center of the BG, with their extensive dendritic arborizations and a large number of dendritic spines (Calabresi et al., 2014; Gerfen and Surmeier, 2011; Kemp and Powell, 1971). GABAergic SPNs receive glutamatergic inputs from the cortex and thalamus as well as DA inputs from the SNc (Fig. 1.5A). Direct pathway SPNs then send their inhibitory projections to output nuclei of the BG, namely the globus pallidus interna (GPi) and SNr, which in turn relay their GABAergic axons to the thalamus. Indirect 
pathway SPNs innervate the globus pallidus externa (GPe), and the inhibitory neurons there project to the STN, GPi, as well as SNc, instead of making direct connections with the thalamus. These output nuclei then send their inhibitory signals to the thalamus (Albin et al., 1989; Calabresi et al., 2014; Ikemoto et al., 2015; Lanciego et al., 2012). The output from the BG to the thalamus is of an inhibitory nature due to the constant GABAergic control originating from the GPi and SNr. Increased thalamic activity occurs when overall GABAergic output signals are reduced and thalamic neurons are disinhibited (Bolam et al., 2000; Chevalier and Deniau, 1990; DeLong, 1990; Kawaguchi, 1997). The BG therefore influence the activity of the thalamus, which projects back to the cortex to complete the cortico-basal ganglia-thalamo-cortical (CBGTC) loop (Bolam et al., 2000; Parent and Hazrati, 1995). DA plays a major role in this circuit. A single SNc DA neuron forms 100,000 to 245,000 synapses and may influence approximately 75,000

A

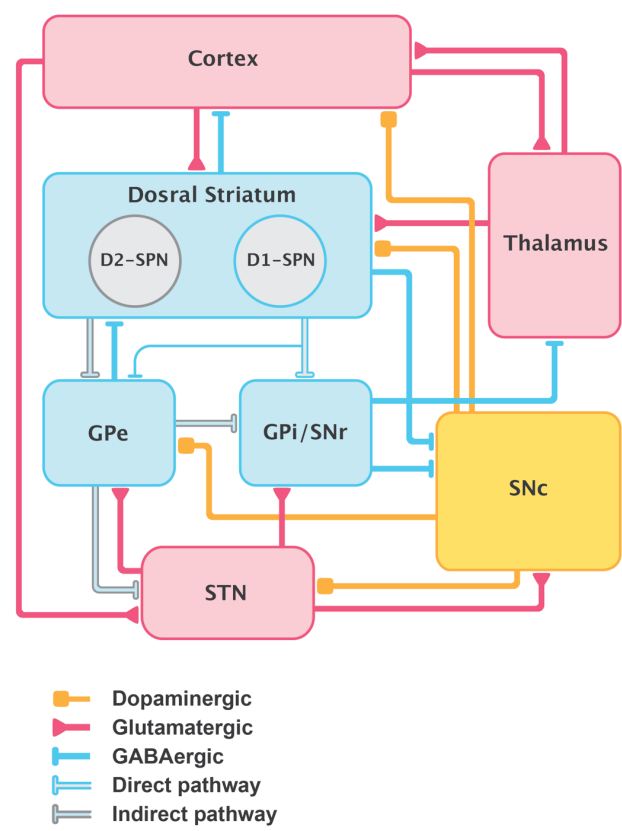

B

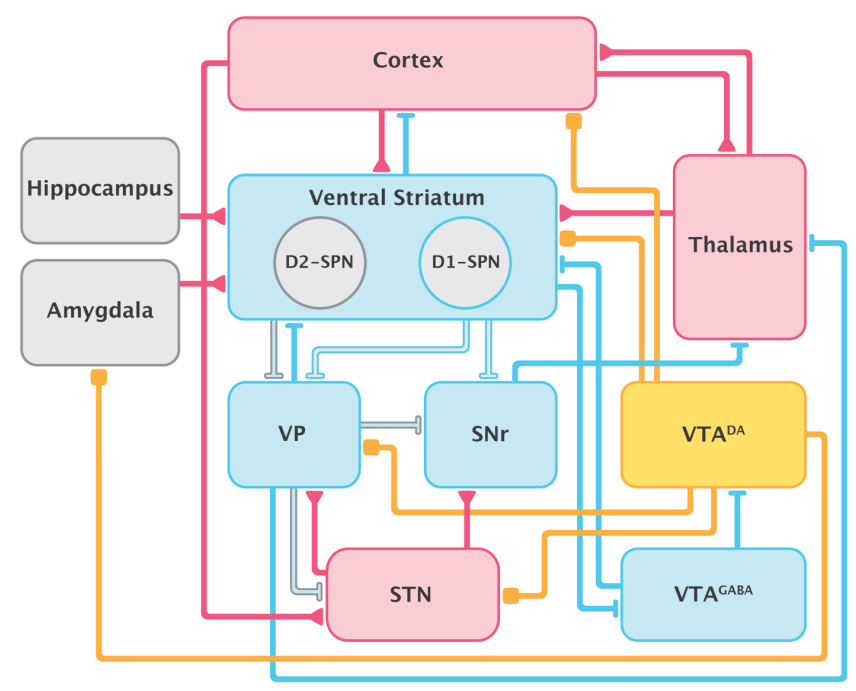

Figure 1.5. Dorsal and ventral basal ganglia circuits. A) Simplified schematic of the dorsal basal ganglia circuits. SNc dopamine neurons play a key role by controlling dopamine levels in the dorsal striatum and maintain the balance between the D1-SPN direct and D2-SPN indirect pathways that control motor behavior. B) Ventral basal ganglia circuits. VTA dopamine neurons project to the ventral striatum which also receives inputs from the cortex and other limbic regions to control cognition and learning. GPe: globus pallidus externa; GPi: globus pallidus interna; SNc: substantia nigra pars compacta; SNr: substantia nigra pars reticulata; SPN: spiny projection neurons; STN: subthalamic nucleus; VP: ventral pallidum; VTA: ventral tegmental area. Based on Cazorla et al., 2015; Humphries and Prescott, 2010; Ikemoto et al., 2015; Kupchik and Kalivas, 2017; Lanciego et al., 2012 \& Mallet et al., 2019. 
neurons in the striatum (Bolam and Pissadaki, 2012; Matsuda et al., 2009). Additionally, DA neurons directly control the activity of the GPi and STN (Smith and Kieval, 2000).

SNc had been suspected as a region of pathology in Parkinson's disease by the early $20^{\text {th }}$ century, and it is in fact the degeneration of nigrostriatal DA neurons that reduces DA in the striatum and causes the motor dysfunctions in Parkinson's disease, for which the primary treatment option is the administration of the DA precursor L-DOPA (Davie, 2008; Goetz, 2011; Lees et al., 2008; Obeso et al., 2010). With emerging anatomical and behavioral evidence, the canonical view of segregated direct and indirect pathways is now shifting to support the notion that the two pathways are intertwined, with multiple transverse loops. For example, $D_{2}$ SPNs were found to form synaptic connections with other $\mathrm{D}_{2}$ SPNs as well as with $\mathrm{D}_{1}$ SPNs, and "bridging" collaterals of SPN axons innervate both the direct pathway SNr and indirect pathway GPe (Calabresi et al., 2014; Cazorla et al., 2015, 2014; Cui et al., 2013; Fujiyama et al., 2011; Lanciego et al., 2012; Parent et al., 2000, 1984; Taverna et al., 2008). These pathways are thus likely coordinating their activation to initiate and select action while suppressing unwanted, competing activity (Calabresi et al., 2014; Cui et al., 2013).

The classic BG model described above strictly applies to the dorsal BG, as the ventral part of the BG bears a different function. The VTA, nucleus accumbens (NAcc), and ventral pallidum (VP) create a "limbic" BG circuitry that mediates cognitive functions such as motivation, reward, aversion, and reinforcement learning. These circuits have been implicated in a range of cognition and reward-related disorders, such as depression, schizophrenia, and addiction (Francis and Lobo, 2017; Macpherson et al., 2014; Root et al., 2015; Smith and Kieval, 2000; Ungless et al., 2010). Similar to the direct and indirect pathways of the dorsal BG, GABAergic SPNs in the NAcc receive inputs from the cortex and other limbic regions such as the hippocampus, amygdala, and VTA (Fig. 1.5B). In the ventral direct pathway, $\mathrm{D}_{1}$-SPNs send their inhibitory projections directly to the output nucleus SNr that inhibits the thalamus, while the ventral indirect pathway $\mathrm{D}_{2}$-SPNs relay their signals to the VP which sends projections to the SNr and thalamus (Geisler and Zahm, 2005; Humphries and Prescott, 2010; Kupchik et al., 2015; Root et al., 2015). The VP also receives afferents directly from VTA DA neurons (Klitenick et al., 1992; Mitrovic and Napier, 2002; Napier, 1992). D1R and D2R in the ventral striatum were thought to be 
associated with the ventral direct and indirect pathways, respectively, but more recent evidence indicated that $\mathrm{D}_{1}$-SPNs and $\mathrm{D}_{2}$-SPNs participate in both pathways and the VP receives projections not only from $\mathrm{D}_{2}$-SPNs but also from $\mathrm{D}_{1}$-SPNs (Kupchik et al., 2015; Kupchik and Kalivas, 2017). Hence, the ventral BG direct and indirect pathways are also likely working together to regulate the thalamus.

To make the BG circuits even more complex, crossovers appear to exist between the dorsal and ventral BG. A recent study combining electrophysiology with retrograde tracing revealed that medial SNc $(\mathrm{mSNc}) \mathrm{DA}$ neurons were heterogeneous in terms of projection sites, sending axons to the dorsolateral striatum (DLS), dorsomedial striatum (DMS), as well as lateral shell of the NAcc (INAcc), while lateral SNc (ISNc) DA neurons project predominantly to the DLS. On the other hand, DA neurons from subregions of the VTA -PBP and PN- innervate predominantly to INAcc and medial NAcc (mNAcc), respectively, revealing the complex topographical organization within the midbrain (Farassat et al., 2019). Furthermore, Haber and colleagues described the feedback and feedforward loops of the midbrain and striatal system as "spirals", where reciprocal projections between subregions of the midbrain and striatum form closed loops, but they also create ascending spirals as each set of axons from striatal subregions project back to the increasingly more dorsal portion of the VTA and SNc. For example, VTA DA neurons project to the ventromedial striatum (VMS)/NAcc shell, which in turn projects back to the VTA but also to the mSNc, while mSNc DA neurons project to the more dorsal part of the VMS/NAcc core, and so on (Haber et al., 2000). The ascending spirals may be key in the development of drug addiction, where the circuit controlling cue-dependent drugseeking behavior transitions from the limbic-oriented ventral BG to the motor-oriented dorsal BG as goal-oriented drug-seeking becomes more habitual (Belin and Everitt, 2008; Everitt and Robbins, 2005). Combining the evidence, the simplified idea is that midbrain DA neurons within the BG are topographically organized in a gradient manner, with more ventromedial -predominantly VTA- subpopulations participating in the mesocorticolimbic pathways and encoding emotion- and cognition-related behaviors, and more dorsolateral -mainly SNc- subpopulations participating in mesostriatal pathways and encoding motor- and action-related functions, and intermediate 
subpopulations may have a single or bridging role in these pathways (Haber, 2014; Haber et al., 2000; Howe and Dombeck, 2016; Poulin et al., 2018). 


\subsection{Intrinsic properties of dopamine neurons}

DA neurons residing in the midbrain can be distinguished on the basis of their morphological and electrophysiological properties from surrounding neurons, with some exceptions in the VTA (Shi, 2009). DA neurons are either multipolar or fusiform in shape, with 2 to 5 major dendrites arising from the soma. In the SNc, those dendrites extend in medial and lateral directions, with one of them often innervating ventrally to the SNr. VTA DA neuron's dendrites extend more radially in comparison (Grace and Onn, 1989; Juraska et al., 1977). The axon of most DA neurons emerges from a dendritic site a few microns to 240 ms from the soma (Grace and Onn, 1989; Häusser et al., 1995). These axonal fibers are usually thin -0.1 to $0.4 \mu \mathrm{m}$ in diameter- and unmyelinated, and densely arborize the striatum (Andén et al., 1966; Bouyer et al., 1984; Braak and Del Tredici, 2004; Matsuda et al., 2009; Pickel et al., 1981). Their total lengths were calculated to be 14 to $78 \mathrm{~cm}$ in rats (Matsuda et al., 2009).

In vivo midbrain DA neurons are spontaneously active with a slow mean firing rate of up to $10 \mathrm{~Hz}$ and a broad action potential waveform $(>2.2 \mathrm{~ms}$ from onset to trough in unfiltered extracellular recordings), as opposed to GABAergic neurons in the region that discharge at a high frequency and have shorter action potential durations (Fig. 1.6) (Grace and Onn, 1989; Koyama and Appel, 2006; Richards et al., 1997). Several factors affect the action potential waveforms of DA neurons: the

Dopamine neuron

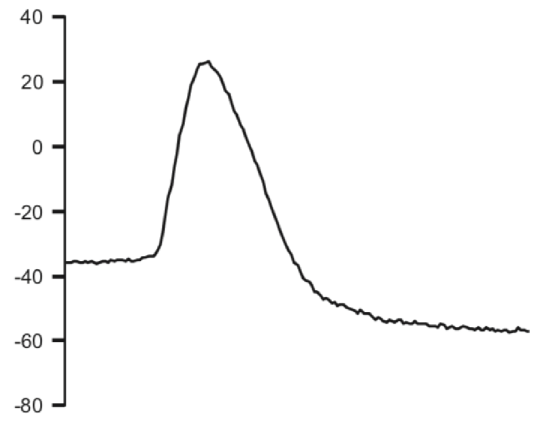

relatively distant

location of the axon initial segment (AIS) from the soma, calcium $\left(\mathrm{Ca}^{2+}\right)$ and potassium $\left(\mathrm{K}^{+}\right)$
GABA neuron

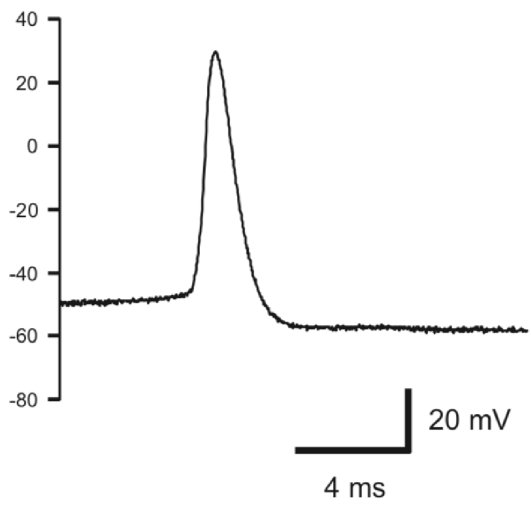

Figure 1.6. Action potential waveforms of dopamine and GABAergic neurons in substantia nigra. Dopamine neurons in substantia nigra pars compacta have much broader action potentials as compared to neighboring GABAergic neurons in substantia nigra pars reticulata. Adapted with permission from "Differences in $\mathrm{Na}^{+}$conductance density and $\mathrm{Na}^{+}$channel function properties between dopamine and GABA neurons of the rat substantia nigra," by V. Seutin and D. Engel, 2010, Journal of Neurophysiology, p. 3101. Copyright (2010) American Physiological Society. 
currents, as well as excitatory synaptic inputs (Häusser et al., 1995; Nedergaard, 1999; Segev and Korngreen, 2007; Shi, 2009). The distance between AIS and soma may also cause a latency from the AIS spike to the somatodendritic spike, which commonly appears as an inflection in the action potential waveform during the rising phase (Grace and Onn, 1989; Häusser et al., 1995).

DA neurons in living animals display two different modes of firing: tonic "pacemaker-like" firing, in which single spikes are interspersed with regular or irregular interspike intervals; phasic "burst" firing, in which a series of spikes occur within a short duration, often with decreasing amplitude (Fig. 1.7) (Grace and Bunney, 1984a, 1984b). Tonic firing maintains the background DA levels at projection sites in the low nanomolar (4 to $20 \mathrm{nM}$ ) range, while phasic signaling transiently elevates the levels to the micromolar range, which leads to increased activation of the low-affinity D1R pathways (Dreyer et al., 2010; Goto and Grace, 2005; Grace, 1991; Liss and Roeper, 2009).

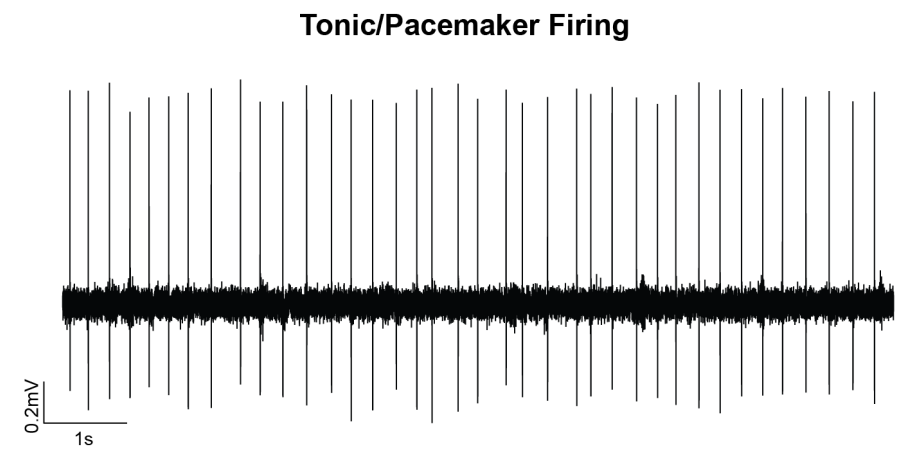

Phasic/Burst Firing

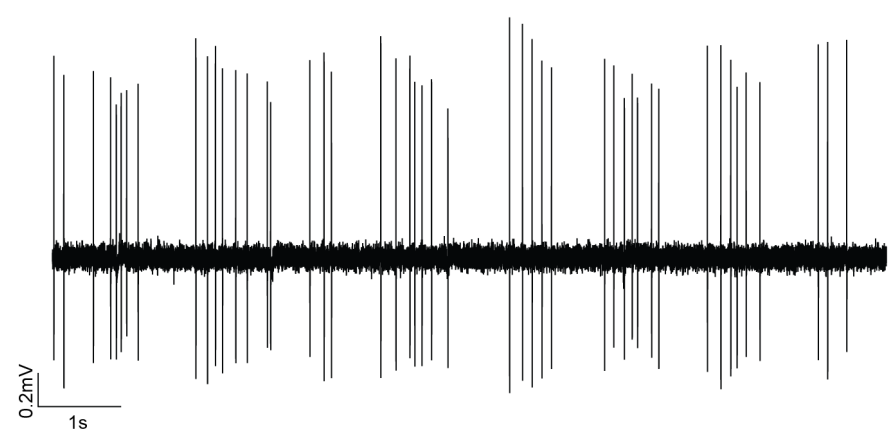

Figure 1.7. Tonic "pacemaker" firing \& phasic "burst" firing of dopamine neurons. Extracellularly-recorded activity of in vivo dopamine neurons. Tonic firing is characterized by highly regular, slow frequency firing. Phasic firing has high frequency "bursts" followed by some non-spiking periods.
Spontaneous bursting in DA neurons occurs only in vivo in the presence of synaptic inputs; in vitro DA neurons display a regular, pacemaking firing pattern with no spontaneous bursts (Grace and Onn, 1989; Sanghera et al., 1984; Shi, 2009). Anthony Grace and Benjamin Bunney published a series of influential studies on in vivo midbrain DA neuron activity in the 1980s, in which they obtained electrical activity from putative rat DA neurons under anesthesia via extracellular and intracellular recording methods combined with fluorescent 
histochemistry. These pioneering studies provided in-depth characterization of morphological and electrophysiological properties of neurochemically-identified in vivo DA neurons that are still relevant today; from the characteristics of their spontaneous firing activity and action potentials described above, to their responses to electrical stimulations and chemical agents, as well as comparisons between extracellular and intracellular observations (Grace and Bunney, 1984a, 1984b, 1983a, 1983b, 1983c, 1980). Importantly, they were the first studies to discuss in vivo burst firing of DA neurons in great detail, and proposed a set of criteria for DA neuron bursting identified based on interspike intervals (ISI); that a burst is said to be initiated when the ISI between two adjacent spikes is shorter than $80 \mathrm{~ms}$ and it terminates when the ISI exceeds $160 \mathrm{~ms}$ (Grace and Bunney, 1984b). This definition of burst firing is still in use today for analyzing in vivo firing activity of extracellularly-recorded DA neurons (Ungless and Grace, 2012). Although a few intracellular studies followed, Grace and Bunney's recordings remain the main source of knowledge in terms of in vivo subthreshold activity and intracellularlyrecorded spontaneous burst firing of DA neurons.

\subsubsection{Control of tonic firing}

Ion channels are pore-forming membrane proteins that allow influx and efflux of ions at rest or in response to stimuli, such as molecule binding or change in voltage (Alexander et al., 2011). Neuronal firing as well as pausing is the result of multiple receptors and ion channels activating and inactivating in coordination. Mechanistic studies of neuronal firing often use the in vitro patch-clamp method in combination with pharmacological reagents to block or allow the flow of specific ionic currents, and much of what is currently known about DA neurons' firing mechanisms comes from such studies.

Tonic firing of DA neurons is characterized by a slowly-depolarizing pacemaking potential that drives the membrane potential to a relatively high action potential threshold -in comparison to GABAergic neighbors and principal neurons in other regions-, and a large afterhyperpolarization (AHP) that follows a broad action potential (Grace and Bunney, 1984a; Grace and Onn, 1989; Tripathy et al., 2015). The composition of ion channels responsible for pacemaker firing varies in distinct DA subpopulations. In 
SNc DA neurons, sodium $\left(\mathrm{Na}^{+}\right)$currents entering through voltage-gated $\mathrm{Na}^{+}(\mathrm{Nav})$ channels generate the fast-spiking component, and tetrodotoxin (TTX) -a neurotoxin found in certain species that specifically blocks $\mathrm{Na}_{v}$ - inhibits spiking in these neurons without disrupting the subthreshold pacemaking oscillations (Fig. 1.8A) (Chan et al., 2007; Grace and Onn, 1989; Kang and Kitai, 1993; Nedergaard and Greenfield, 1992; Yang et al., 2019). In both SNc and VTA, Nav densities are 4.5 to 9-fold higher in axon initial segments (AIS) where action potentials originate in comparison to somas, and AIS $\mathrm{Nav}$ (mostly Nav1.2) inactivates and recovers much quicker than that of soma in order to ensure spiking (Yang et al., 2019). The density of somatic $\mathrm{Nav}$ is relatively low in DA neurons, which renders them susceptible to depolarization block with increased firing rate, thus $\mathrm{Na}_{\mathrm{v}}$ limits the maximal firing frequency of DA neurons (Seutin and Engel, 2010; Tucker et al., 2012). Maximal amplitudes of somatic voltage-gated $\mathrm{Na}^{+}$currents in pyramidal neurons are about ten-fold higher in comparison (Hu et al., 2009; Yang et al., 2019).

A

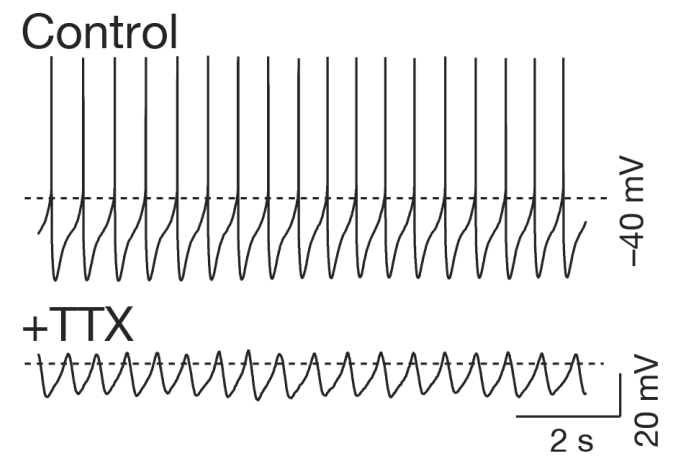

B

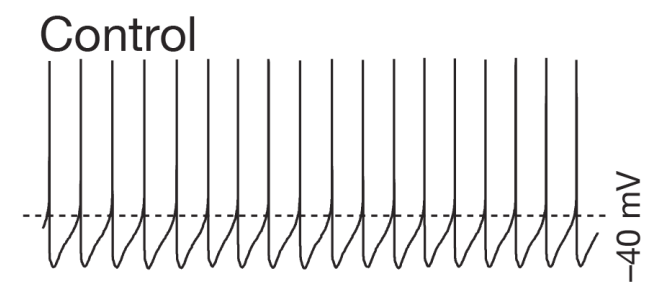

+Nimodipine

Figure 1.8. Voltage-dependent L-type $\mathrm{Ca}^{2+}$ channels drive pacemaker oscillations in dopamine neurons. A) In vitro substantia nigra pars compacta dopamine neurons are devoid of action potentials in the presence of voltage-gated sodium channel blocker tetrodotoxin, but subthreshold pacemaker oscillations are mostly intact. B) Voltage-dependent L-type calcium channel blocker nimodipine abolishes pacemaker oscillations of dopamine neurons. TTX: tetrodotoxin. Adapted with permission from "'Rejuvenation' protects neurons in mouse models of Parkinson's disease," by C.S. Chan et al., 2007, Nature, p. 1082. Copyright (2007) Springer Nature.

Another channel crucial for SNc DA neuron pacemaking is the voltage-dependent L (long-lasting)-type calcium ( $\mathrm{Ca}^{2+}$ ) channel, which mediates the oscillations underlying pacemaking potentials. Application of the L-type $\mathrm{Ca}^{2+}$ channel blocker nimodipine 
inhibits this component altogether and abolishes spiking (Fig. 1.8B) (Chan et al., 2007; Grace and Onn, 1989; Kang and Kitai, 1993; Nedergaard and Greenfield, 1992). Chan and colleagues claimed that, in juvenile SNc DA neurons, voltage-dependent $\mathrm{Na}^{+}$channels and hyperpolarization-activated cyclic nucleotide-gated (HCN) cation channels drive pacemaker firing, until L-type $\mathrm{Ca}^{2+}$ channels replace $\mathrm{HCN}$ channels as the voltage range of $\mathrm{I}_{\mathrm{H}}$ shifts to more negative voltages with increasing age. The same study postulated that SNc DA neurons' heavy dependence on Cav1.3 for autonomous pacemaker activity may make them vulnerable to Parkinson's disease as it can elevate intracellular $\mathrm{Ca}^{2+}$ concentration with age (Chan et al., 2007). Along with $\mathrm{Cav} 1.3$, another $\mathrm{Ca}^{2+}$ channel contributing to Parkinson's disease is the R-type voltage-dependent $\mathrm{Ca}^{2+}$ channel Cav2.3. It was found to be the most abundant $\mathrm{Ca}_{v}$ in adult SNc DA neurons as compared to Cav1.2 and $\mathrm{Cav}_{\mathrm{v}} 1.3$, and participates in the pacemaking $\mathrm{Ca}^{2+}$ oscillations without affecting firing frequency (Benkert et al., 2019). Cav1.2 and Cav1.3 are both part of the same high voltageactivated (HVA) group, but Cav1.3 is activated at much more hyperpolarized membrane potentials as compared to Cav1.2 (Benarroch, 2010; Chan et al., 2007; Philippart et al., 2016; Xu and Lipscombe, 2001). Other $\mathrm{Ca}^{2+}$ channels such as the $\mathrm{N}$-type and $\mathrm{P} / \mathrm{Q}$-type are also present in midbrain DA neurons, but they do not appear to significantly affect pacemaker firing (Cardozo and Bean, 1995; Durante et al., 2004).

In vitro tonic firing of VTA DA neurons is either regular or slightly irregular and is primarily mediated by $\mathrm{Nav}$ and HCN channels, similar to juvenile SNc DA neurons (Chan et al., 2007; Khaliq and Bean, 2010). HCN channels mediate a prominent inward rectification $\left(\mathrm{I}_{\mathrm{H}}\right)$, also known as sag, observed during hyperpolarizing

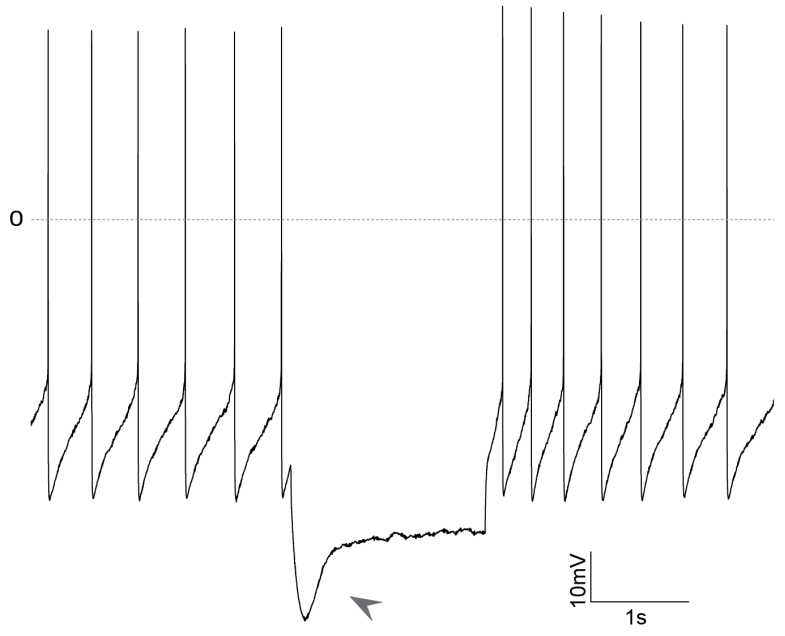

Figure 1.9. Sag component displayed by in vitro dopamine neuron upon hyperpolarization. Hyperpolarization-activated cyclic nucleotide-gated (HCN) channels mediate a prominent inward rectification known as sag (gray arrow). Because many dopamine neurons exhibit this property when hyperpolarized, it can be used to identify those "conventional" dopamine neurons. In vitro recording trace courtesy of Josef Shin. 
current injections in the majority of DA neurons (Fig. 1.9) (Grace and Bunney, 1983b; Grace and Onn, 1989; Kita et al., 1986; Neuhoff et al., 2002). Classically, the presence of sag was thought to indicate DA neuron identity in the midbrain, but studies indicate that not all DA neurons display the sag component. At least in vitro, these 'conventional' DA neurons that showed prominent sag comprised the DA subpopulation projecting to the striatum and INAcc, while sag was less pronounced or even absent in the rest of mesocorticolimbic (or 'atypical') DA subpopulation as well as in non-DA neurons in the region (Koyama and Appel, 2006; Lammel et al., 2008; Zhang et al., 2010). HCN currents help maintain pacemaker firing by depolarizing the membrane potential toward baseline in response to hyperpolarization (Lammel et al., 2008; Lüthi and McCormick, 1998; Neuhoff et al., 2002). Application of an HCN channel blocker ZD 7288 does not abolish pacemaker firing as with TTX or nimodipine, but it slows down spiking in the calbindinnegative SNc DA subpopulation (Chan et al., 2007; Neuhoff et al., 2002).

The voltage-dependent A-type $\mathrm{K}^{+}$channel also controls the rate of firing in DA neurons. Named after the shape of its current kinetics, the A-type $\mathrm{K}^{+}$channel is activated when the rate of depolarization becomes greater than $10 \mathrm{mV} / \mathrm{s}$, which leads to fast, transient and large amplitude $\mathrm{K}^{+}$currents $\left(\mathrm{I}_{\mathrm{A}}\right)$ that results in a slower rate of depolarization (Khaliq and Bean, 2008; Koyama and Appel, 2006; Liss et al., 2001; Tarfa et al., 2017). While $\mathrm{K}_{\mathrm{V}} 4.3 \mathrm{~L}$ (long splice variant; also known as KCND3) is the only alpha subunit expressed in SNc, $\mathrm{I}_{\mathrm{A}}$ is observed in both SNc and VTA DA neurons with varying inactivation rates, with much slower inactivation kinetics in mesoaccumbal DA neurons (Koyama and Appel, 2006; Liss et al., 2001; Tarfa et al., 2017). $\mathrm{I}_{\mathrm{A}}$ kinetics change the duration of rebound delay, i.e. time interval between the termination of somatic hyperpolarizing current injection and the first spike. The slow inactivation kinetics of mesoaccumbal DA neurons with decay time constants of up to $225 \mathrm{~ms}$ lead to rebound delays twice as long as those of nigrostriatal DA neurons on average (Tarfa et al., 2017). It turns out that when DA neurons receive GABAergic inputs, A-type $\mathrm{K}^{+}$channels work to prolong the duration of GABAergic inhibition, which, based on computational models, may be shortened in the presence of $\mathrm{I}_{\mathrm{H}}$ or $\mathrm{T}$ (transient opening)-type $\mathrm{Ca}^{2+}$ currents, although not very effectively when $I_{A}$ inactivation is slow (Tarfa et al., 2017). Blocking the A-type channel with the application of the giant crab spider venom and a $\mathrm{K}_{v} 4$-specific 
blocker heteropodatoxin-3 (HpTx3) increases firing rates of SNc DA neurons in a dosedependent manner (Koyama and Appel, 2006; Liss et al., 2001; Tarfa et al., 2017).

\subsubsection{Control of phasic firing}

Biophysical mechanisms of phasic firing are not as well understood in comparison to those of tonic firing due to the technical difficulty that limits direct access to DA neuron's intrinsic activity in vivo. Nevertheless, a few ion channels and receptors have been identified as likely candidates of phasic firing in DA neurons. The voltage-dependent $\mathrm{T}$ (transient opening)-type $\mathrm{Ca}^{2+}$ channel and small conductance $\mathrm{Ca}^{2+}$-activated potassium $\left(\mathrm{K}^{+}\right)$channel 3 (SK3) are a set of ion channels that regulate pacemaker firing in SNc DA neurons that may also play a part in burst firing (Wolfart et al., 2001; Wolfart and Roeper, 2002). The SK3 channel forms a complex with $\mathrm{Ca}^{2+}$ sensor calmodulin and is activated by increased influx of $\mathrm{Ca}^{2+}$ mainly via $\mathrm{T}$-type $\mathrm{Ca}^{2+}$ channels during an action potential (Köhler et al., 1996; Wolfart et al., 2001; Wolfart and Roeper, 2002; Xia et al., 1998). The resulting outward $\mathrm{K}^{+}$current creates a large AHP which reverses directions to slowly depolarize the membrane potential, initiating another action potential cycle (Shepard and Bunney, 1991; Wolfart et al., 2001; Wolfart and Roeper, 2002). The $\mathrm{K}^{+}$currentmediated medium AHP is abolished when apamin, a bee venom and a selective SK channel blocker is administered, which results in increased firing rates as well as irregular firing pattern, and in some cases burst firing in SNc DA neurons in vitro (Shepard and Bunney, 1991; Wolfart et al., 2001; Wolfart and Roeper, 2002). A recent modeling study proposed that, with suppressed SK channel-mediated AHP, some voltagegated $\mathrm{Na}^{+}$channels stay inactivated rather than recover, causing the neuron to fire in irregular patterns (Iyer et al., 2017). Furthermore, when the upstream T-type $\mathrm{Ca}^{2+}$ channels are inhibited by nickel $\left(\mathrm{Ni}^{2+}\right)$, a non-selective $\mathrm{Ca}^{2+}$ channel blocker but sensitive for low-voltage activated (LVA) T-type channels, DA neurons fire in burst-like manners followed by a prominent hyperpolarization (Fig. 1.10). Application of both apamin and $\mathrm{Ni}^{2+}$ cause even more pronounced bursting in SNc DA neurons (Wolfart and Roeper, 2002). These bursts appear to be facilitated by N-methyl-D-aspartate (NMDA) receptors (NMDAR) and are terminated when the $\mathrm{Na}^{+} / \mathrm{K}^{+}$-adenosine triphosphatase (ATPase) generates an outward current leading to hyperpolarization and $\mathrm{Mg}^{2+}$ block of NMDA 


\section{Control}

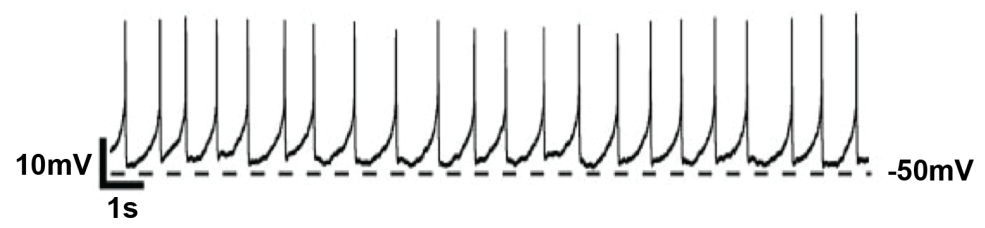

$100 \mu \mathrm{M}$ Nickel

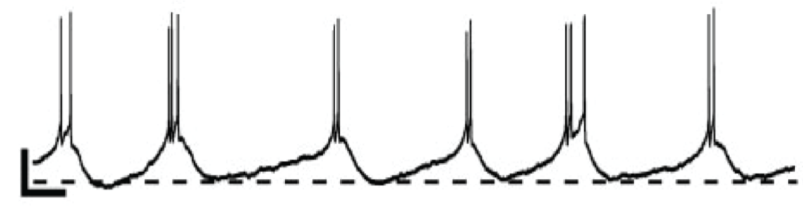

Figure 1.10. Blocking T-type $\mathrm{Ca}^{2+}$ channels induce burst firing of in vitro dopamine neurons. Voltage-dependent T-type calcium channels are blocked in the presence of nickel, which leads to burst firing in dopamine neurons in vitro. Adapted from "Selective coupling of T-type calcium channels to SK potassium channels prevents intrinsic bursting in dopaminergic midbrain neurons," by Copyright (2002) Society for Neuroscience. J. Wolfart and J. Roeper, 2002, Journal of Neuroscience, p. 3410.

receptors (Johnson et al., 1992). Interestingly, activation rather than inactivation of T-type $\mathrm{Ca}^{2+}$ channels induces rhythmic bursting in other systems such as thalamic (Cain and Snutch, 2010; Deschênes et al., 1984), lateral and medial habenula neurons

(Vickstrom et al., 2020; Wilcox et al., 1988), suggesting that $\mathrm{T}$-type $\mathrm{Ca}^{2+}$ channels function differently in DA neurons (Wolfart and Roeper, 2002).

Our lab's previous study found that adenosine triphosphate-sensitive $\mathrm{K}^{+}$(K-ATP) channels to be essential for rhythmic burst firing in DA neurons located in mSNc using in vivo extracellular recording combined with juxtacellular labeling in K-ATP knockout ( $\mathrm{K}_{\mathrm{ir}} 6.2 \%$ ) animals (Schiemann et al., 2012). DA neurons in ISNc also express K-ATP channels but their firing is not affected by altered functioning of K-ATP channels (Liss et al., 2005; Schiemann et al., 2012). Furthermore, burst activity observed in mSNc DA neurons requires both K-ATP channel as well as NMDAR activation, as an administration of K-ATP channel inhibitor tolbutamide terminates induced bursting in vitro. Surprisingly, in the absence of NMDA, activation of K-ATP channels with NN414, the sulfonylurea receptor 1 (SUR1; regulatory subunit of K-ATP)-selective agonist, slows down DA neuron firing (Schiemann et al., 2012). K-ATP channel-mediated burst firing is necessary for novelty-induced exploration, as silencing mSNc K-ATP results in reduced novelty exploration (Schiemann et al., 2012). A follow-up computational study proposed that burst firing of mSNc DA neurons involves $\mathrm{Ca}^{2+}$ influx which activates K-ATP by local 
accumulation of adenosine diphosphate (ADP), and hyperpolarization of membrane potentials by GABAergic inputs is necessary for burst termination (Knowlton et al., 2018).

GABAergic inhibition and disinhibition may indeed play an essential role in burst firing of DA neurons, as microscopic analysis found that about $70 \%$ of synaptic inputs onto the SNc are GABAergic (Bolam and Smith, 1990). The $\mathrm{GABA}_{\mathrm{A}}$ receptor is an ionotropic receptor which upon activation opens its chloride ( $\left.\mathrm{Cl}^{-}\right)$channel to hyperpolarize the membrane potentials, whereas the GABAB receptors are G-proteincoupled metabotropic receptors that when activated open $\mathrm{K}^{+}$channels as well as reduce $\mathrm{Ca}^{2+}$ conductance, leading to inhibition (Enna, 2007). The two GABA receptors evoke different inhibitory responses in DA neurons: $\mathrm{GABA}_{\mathrm{A}}$ receptors are responsible for early inhibition, while $\mathrm{GABA}_{B}$ receptors mediate late inhibition (Brazhnik et al., 2008). In vivo extracellular recording revealed that striatal, GP, and SNr GABAergic projections to DA neurons work via $G_{A B A_{A}}$ receptors since electrical stimulation of those pathways inhibited DA neurons. Local application of $\mathrm{GABA}_{\mathrm{A}}$ antagonists bicuculline or picrotoxin attenuated or blocked the inhibition of DA neurons, leading to increased burst firing (Paladini et al., 1999; Paladini and Tepper, 1999). In addition, antidromic activation of SNr GABAergic neurons that inhibits SNc DA neurons can be blocked by bicuculline application in the SNc, which switches DA neurons into burst firing, while administration of the $\mathrm{GABA}_{\mathrm{B}}$ receptor antagonist saclofen results in increased pacemaker firing (Tepper et al., 1995). However, $G A B A_{B}$ receptors may induce burst firing by a different mechanism; for example, by suppressing the small-conductance $\mathrm{Ca}^{2+}$-activated $\mathrm{K}^{+}(\mathrm{SK})$ channel, which was shown to increase firing rate and irregularity in SNc DA neurons (Estep et al., 2016). Disinhibition of glutamatergic and cholinergic projections from the pedonculopontine tegmental nucleus (PPTg) by $\mathrm{GABA}_{\mathrm{A}}$ receptor blockade was also found to increase burst firing in SNc DA neurons. (Floresco et al., 2003; Galtieri et al., 2017). Additionally, burst firing of SNc DA neurons may also involve the $\alpha$-amino-3-hydroxy-5methyl-4-isoxazolepropionic acid receptor (AMPAR) and both nicotinic and muscarinic cholinergic receptors (Lee and Tepper, 2009). 


\subsubsection{Control of inhibition}

Apart from GABAergic receptors discussed above, a few ion channels have been implicated in suppressing the activity of DA neurons by decreasing excitability, such as the $\mathrm{G}$ protein-coupled inwardly-rectifying $\mathrm{K}^{+}$channel (GIRK; also known as $\mathrm{K}_{\mathrm{ir}} 3$ ) and voltage-gated $\mathrm{K}^{+}$channel $\mathrm{K}_{\mathrm{V}}$ 7, also referred to as KCNQ from its encoding gene. GIRK channels are a downstream target of a wide variety of G protein-coupled receptors such as $\mathrm{GABA}_{\mathrm{B}}$ receptors and D2R (Arora et al., 2011; Cruz et al., 2004; Lacey et al., 1987). The inhibitory effect of D2R is mediated by GIRK channels, because activation of D2R leads to the binding of the G $\beta \gamma$ subunit of G protein to GIRK channel, which results in the generation of outward $\mathrm{K}^{+}$current and thus hyperpolarization of the membrane potential (Beaulieu and Gainetdinov, 2011; Ford, 2014; McCall et al., 2017). Pharmacological studies of GIRK channel have mostly used non-selective $\mathrm{K}^{+}$channel blockers due to the relative unavailability of selective blockers, but GIRK channel appears to be sensitive to psychostimulants (Rifkin et al., 2017; Walsh, 2011). Exposure to cocaine or methamphetamine was found to reduce GIRK currents mediated by both GABA receptors and D2R in VTA DA neurons, for which the GIRK2/GIRK3 heteromeric channel is responsible (Arora et al., 2011; Kotecki et al., 2015; McCall et al., 2019; Sharpe et al., 2014). In addition, D2R- and GABAB-mediated GIRK currents are differentially affected by intracellular $\mathrm{Ca}^{2+}$. Weak $\mathrm{Ca}^{2+}$ buffering results in faster desensitization of D2Rdependent GIRK signaling (Gantz et al., 2015).

The non-inactivating $\mathrm{K}_{\mathrm{V}} 7$ channels generate steady $\mathrm{K}^{+}$currents at subthreshold membrane potentials to exert their influence on the baseline excitability of the neuron. They are also called M-channels because their 'M-currents' are suppressed by activation of muscarinic acetylcholine receptors (mAchR) (Brown and Passmore, 2009; Hansen et al., 2008). In recent studies, the KV7.4 channel was found to contribute to D2R-mediated inhibition in NAcc-projecting VTA DA neurons, along with GIRK (Li et al., 2017; Su et al., 2019). Another KCNQ channel containing the $K_{V} 7.3$ subunit (also known as KCNQ3) was shown to promote resilience after chronic social defeat by increasing its current to reduce hyperexcitability caused by chronic social defeat-induced rise in $\mathrm{I}_{\mathrm{H}}$ in VTA DA neurons. (Friedman et al., 2016, 2014). 
In addition to being controlled by glutamatergic and GABAergic inputs as well as DA itself, DA neurons receive neuromodulatory inputs such as acetylcholine, serotonin, oxytocin, and other neuropeptides that also modulate the activity of DA neurons and thus DA levels at projection sites (De Deurwaerdère and Di Giovanni, 2017; Margolis et al., 2014; Peris et al., 2017; Xiao et al., 2020, 2017). Each of these channels and receptors is differentially expressed in DA neurons, and while some subpopulations share certain molecular profiles, these differences in expression create the diverse electrical responses displayed by DA neurons (Tapia et al., 2018). This diversity may enable DA neurons to fulfill their versatile roles in a wide range of behaviors that they code. 


\subsection{Functions of midbrain dopamine neurons}

The previous section covered the intrinsic and extrinsic mechanisms of midbrain DA neurons that control firing patterns, which in turn regulate extracellular DA levels. Although my project did not involve behavioral experiments, the significance of studying electrophysiological properties of midbrain DA neurons lies in understanding how they integrate converging inputs and communicate information to downstream neurons that ultimately results in behavior. As part of the BG, DA circuits are at the core of some fundamental behaviors. One such behavior and one of the most well-known functions of the BG is motor control. Studies have reported phasic increases of SNc DA neuron activity before the initiation and during acceleration of movement (da Silva et al., 2018; Howe and Dombeck, 2016). Degeneration of SNc DA neurons in Parkinson's disease may lead to disruption of this type of rapid and precisely-timed control of the $\mathrm{BG}^{\prime} \mathrm{s} \mathrm{D}_{1}$ - and $\mathrm{D}_{2}$ pathways, which results in motor impairment that manifests as tremors, rigidity, and akinesia (Braak and Del Tredici, 2004; Calabresi et al., 2014; Goetz, 2011; Obeso et al., 2010). Reinforcement learning, motivation, and habit formation have also been identified as behaviors under the control of DA (Salamone and Correa, 2012; Schultz et al., 1997; Yin and Knowlton, 2006). Enabled by the recent development and growing availability of novel investigative tools such as $\mathrm{Ca}^{2+}$ imaging, optogenetics, chemogenetics, and neuronal tracing, genetically-targeted studies of DA pathways in behaving animals in the last decades have been particularly informative and are uncovering the level of complexity of these circuits that underlie behavior, leading us to revise our understanding about the functions of the DA system (Lammel et al., 2014). In this section, I will briefly discuss behaviorally-relevant DA signaling and circuits, focusing on reinforcement learning.

\subsubsection{Prediction error coding of dopamine neurons}

Along with motor control, reward and reward-based learning is one of the most researched functions of the DA system. In the context of behavioral research, reward is considered a positive reinforcer that encourages a particular behavior that is expected to maximize the chance of obtaining the reward, whether it be an object, mate, food, or inner state. Punishment on the other hand works as an aversive or negative reinforcement 
signal where animals seek to minimize the chance of receiving the punishment. This reinforcement learning is thought to be driven by prediction error, i.e. deviation of the outcome from the prediction (Nasser et al., 2017; Schultz et al., 1997; Watabe-Uchida et al., 2017). Researchers had observed behavioral reinforcement by striatal electrical stimulation and phasic activity of midbrain DA neurons in response to reward and reward-associated cues already in the 1950s. It was however the monumental work by Schultz and colleagues that demonstrated the role of midbrain DA neurons in prediction error coding with a series of experimental work, which was then applied to the computational models developed earlier by psychologists (Crow, 1972; Miller et al., 1981; Nasser et al., 2017; Olds and Milner, 1954; Rescorla and Wagner, 1972; Schultz et al., 1997; Sutton and Barto, 1981; Watabe-Uchida et al., 2017).

In single-unit extracellular recordings of putative DA neurons in the midbrain of naïve monkeys, a majority of DA neurons would increase firing when receiving an unexpected reward (Fig. 1.11A). After monkeys had been subjected to classical conditioning, in which they were repeatedly trained to associate a neutral conditioned stimulus (CS) -such as a tone or light- with a rewarding unconditioned stimulus (US) such as chocolate- which consistently followed CS, their DA neurons in SNc and VTA fired in a phasic mode not after the reward delivery but at the presentation of CS (Fig. 1.11B). Moreover, when the expected reward was missing after the presentation of CS, DA

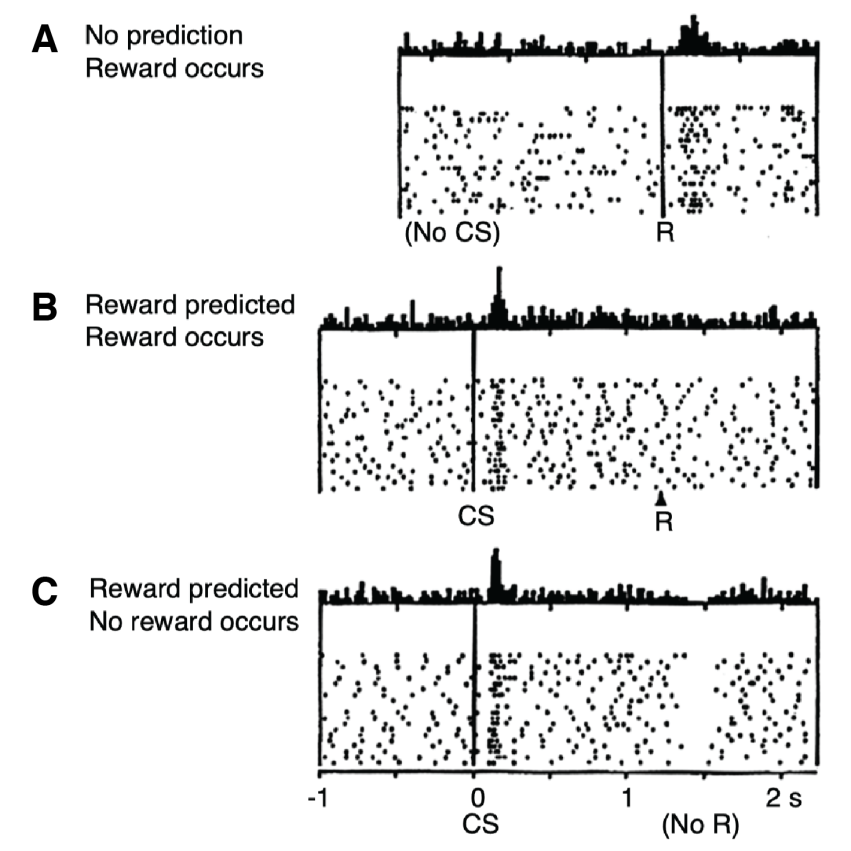

Figure 1.11. Prediction error coding of dopamine neurons. A) In naïve monkeys, phasic firing of dopamine neurons occurs after the presentation of reward. B) After being conditioned, dopamine neurons of monkeys fire at the presentation of the cue which reliably predicts subsequent reward, but not at the presentation of reward itself. C) After being conditioned, dopamine neurons decrease firing if the predicted reward is missing after the presentation of the predictive cue. CS: conditioned stimulus; R: reward. From "A neural substrate of prediction and reward," by W. Schultz, P. Dayan and P.R. Montague, 1997, Science, p. 1594. Reprinted with permission from AAAS. 
neuron activity was suppressed (Fig 1.11C). Applying these experimental results to the temporal difference (TD) learning model developed by Sutton and Barto, Schultz proposed that DA neurons code the quantitative error between the predicted value and timing of future rewarding events and the actual outcome (i.e. presence or absence, value and temporal information), where a positive error evokes phasic firing and a negative error decreases firing, and no change in firing results when experience fully matches prediction. In addition, the quantitative value of reward, which is represented by phasic firing of DA neurons, becomes transferred to the earliest sensory cue that would reliably predict future rewarding stimuli. Prediction error coding of DA neurons thus serves as a bidirectional teaching signal to update predictions and modify behavior and to reinforce model-free learning according to positive and negative experiences (Hollerman and Schultz, 1998; Nasser et al., 2017; Schultz et al., 1997; Sutton and Barto, 1981). Numerous subsequent studies have replicated prediction error coding in DA neurons and demonstrated that the outcome of reinforcement learning can be controlled by direct manipulation of VTA DA neurons to artificially elicit positive and negative prediction errors (Chang et al., 2016; Nasser et al., 2017; Stauffer et al., 2016; Steinberg et al., 2013; Tsai et al., 2009). Moreover, it was recently shown that DA neurons appear to integrate the converging signals about reward and expectation from multiple brain regions such as the striatum, LH, STN, and PPTg, as well as local GABAergic neurons to compute their prediction error signals (Eshel et al., 2015; Tian et al., 2016).

The theory of prediction error coding continues to be updated and expanded based on new experimental evidence. It was initially thought that DA neurons encoded incentive stimuli and reward prediction error and that aversive stimuli would either suppress or not alter DA neuron activity, as Schultz had shown. While this has been a subject of debate, compelling evidence now indicates that at least certain populations of DA neurons in the VTA are excited by aversive events such as airpuff or footshock (Brischoux et al., 2009; Bromberg-Martin et al., 2010; de Jong et al., 2019; Menegas et al., 2018; Ungless et al., 2004). Bromberg-Martin and colleagues hypothesized that two distinct types of DA neurons signal either motivational value, such that they are activated or suppressed by stimuli, or motivational salience, such that they are activated by motivationally-relevant cues, and in addition, both types encode 'alerting' signals that 
grab the animals' attention (Bromberg-Martin et al., 2010). However, recent work by De Jong et al. demonstrated that DA neurons activated by aversive stimuli indeed belong to a distinct projection-specific DA subpopulation by showing via fiber photometry that DA terminals in the ventral mNAcc are activated by unexpected aversive stimuli and associated cues while DA terminals in other NAcc regions are excited by unexpected reward and reward-linked cues and inhibited by aversive events (de Jong et al., 2019). Furthermore, in an elegantly-designed series of conditioning experiments combined with optogenetics, Sharpe and colleagues provided evidence that mesolimbic DA signaling is sufficient and necessary for association of cues even in the absence of reward, and argued that prediction errors of DA neurons can also promote model-based learning that is not based on empirically-obtained cached value as proposed by Schultz (Sharpe et al., 2017). Although their optogenetic manipulation was not targeted to specific projection-defined DA neurons like in De Jong's study, Sharpe's work significantly expanded the concept of DA's prediction error coding to include more general associative learning regardless of value, which suggests that DA transients may play a much bigger role in learning than originally thought (Langdon et al., 2018; Sharpe et al., 2017). 


\section{AIM OF THE PROJECT}

Given the gap in the current knowledge of biophysical mechanisms of phasic firing in midbrain DA neurons in vivo, my project aims to characterize subthreshold membrane activity that drives tonic versus phasic firing of midbrain DA neurons in the intact brain.

In order to achieve this goal, I set three objectives: 1) to establish the deep-brain in vivo patch-clamp method in anesthetized C57BL/6 mice; 2) to record spontaneous firing activity of putative DA neurons in the midbrain and confirm their identity; 3) to establish novel analysis methods to extract meaningful subthreshold membrane information in relation to firing patterns.

As an additional goal, I aim to establish a map to describe subthreshold membrane behavior of in vivo DA neurons as a population that can be used as a guide for future experiments. 


\section{MATERIALS \& METHODS}

\subsection{Animals}

Animals used for the experiments described in this thesis were $\mathrm{C} 57 \mathrm{BL} / 6 \mathrm{~N}$ and C57BL/6J male mice aged 8-16 weeks obtained from Charles River Laboratories (Germany) and Janvier Labs (France), respectively. Mice were housed in plastic cages and maintained on a 12-hour light/dark cycle with food (ssniff, Germany) and water available ad libitum. All experimental procedures were approved by and conducted in accordance with the German Regional Council of Darmstadt (TVA 54-19c20/15-F40/28).

\subsection{Head-plate implantation}

Prior to surgery, animals were intraperitoneally injected with $50 \mu \mathrm{L}$ atropine to reduce mucus secretions. Mice were initially placed in a chamber for anesthesia induction with isoflurane (Forane, AbbVie) then moved to a stereotaxic frame (David Kopf Instruments) and maintained under anesthesia with a constant flow of isoflurane (1.0$2.5 \%$ in $100 \% 02,0.35 \mathrm{~L} / \mathrm{min}$ ) for the duration of surgery. Breathing rate was monitored and body temperature was kept at $37-38{ }^{\circ} \mathrm{C}$ using a custom electronic heating blanket. To avoid corneal damage, animals' eyes were covered with ophthalmic ointment (Vidisic Gel, Bausch+Lomb). Anti-inflammatories dexamethasone $(0.5 \mu \mathrm{L} / \mathrm{g}$ body weight; Dexaratiopharm, ratiopharm) and carprofen $(0.08 \mu \mathrm{L} / \mathrm{g}$ body weight; Rimadyl, Zoetis) were administered subcutaneously to the neck. Lidocaine (Emla, Aspen) was applied to the scalp as a local analgesia prior to making incision. A circular incision (approx. $1 \mathrm{~cm}$ diameter) was then made to the head to reveal the parietal bones as well as lambda and bregma. After the skull was cleared of the periosteum and cleaned with saline, it was leveled using a Stereotaxic Alignment Indicator (David Kopf Instruments). As craniotomies would be performed days later, reference indentations were made above the VTA (AP: $-3.08 \mathrm{~mm}$, ML: $\pm 0.25-0.75 \mathrm{~mm}$ ) and SNc (AP: -3.08 mm, ML: 0.9-1.4 mm) with a Stereotaxic Drill (David Kopf Instruments). The skull surface was then thoroughly scratched with a bone scraper to create a rough texture. A hand drill with a $0.6 \mathrm{~mm}$ drill bit was used to create two holes in the skull: one in the right frontal bone and the other in the interparietal bone. Screws (SS bone screw, BASi) were then partially inserted into 
the holes and super glue (Loctite) was applied around them to strengthen the grip. After glue had dried, a custom head-plate was lowered to the skull with a custom holder and super glue (Loctite) was applied to set the head-plate in place (Fig. 3.1). Dental cement (Paladur, Kulzer) was subsequently applied around the head-plate and screws to firmly anchor the head-plate to the skull while keeping the future craniotomy site clean. After a few minutes, a live tissue adhesive (Kwik-Sil, World Precision Instruments) was applied to protect the area that was not covered by dental cement.

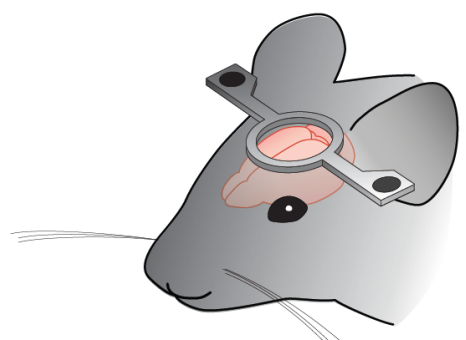

Figure 3.1. Placement of a head-plate.

\subsection{Retrograde tracer infusion}

To label DA neurons from their axonal projection sites, Fluoro-Gold (FG) was infused into the INAcc or mNAcc 7-10 days before recording (Fig. 3.2). FG is a retrograde neuronal tracer that accumulates in lysosomes and gets transported to the soma (Ferguson, 2018; Persson and Havton, 2009; Schmued et al., 1989). FG (Fluorochrome) was dissolved in artificial cerebrospinal fluid (ACSF) to make a concentration of $0.002 \%$,

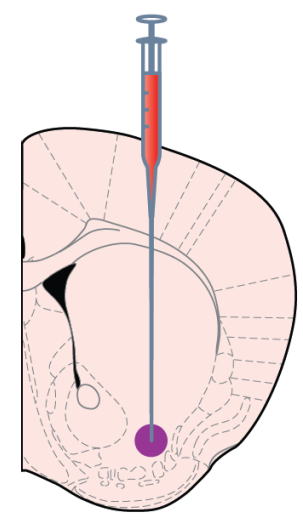

INAcC

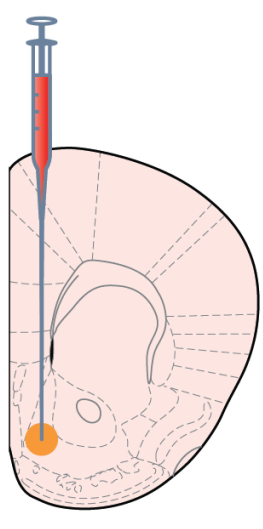

mNAcc

Figure 3.2. Fluoro-Gold infusion sites. INAcc: nucleus accumbens lateral shell; mNAcc: nucleus accumbens medial shell. Brain illustrations adapted with permission from "The Mouse Brain in Stereotaxic Coordinates, 3rd edition," by G. Paxinos and K. Franklin, 2007. Copyright (2007) Elsevier.

which was found to cause no structural damage nor alteration of spiking in DA neurons (Farassat et al., 2019; Wessendorf, 1991). Prior to infusion, thin glass micropipettes (G120F-4, Warner Instruments) were prepared with a pipette puller (DMZ Universal Electrode Puller, Zeitz-Instruments) and filled midway with mineral oil, followed by Millipore water. $1-\mu \mathrm{L}$ microliter syringe (Hamilton Company) was inserted halfway into the pipette, and the pipette was subsequently tightly secured to the syringe using a hot glue gun. Infusion sites were determined based on Paxinos and Franklin's coordinates (Paxinos and Franklin, 2007). 
FG infusion was carried out simultaneously as head-plate implantation surgery, after leveling the skull but before marking the SNc or VTA. After the skull had been leveled, bilateral craniotomies were performed above the INAcc (AP: $0.86 \mathrm{~mm}$, ML: 1.75 $\mathrm{mm}$ ) or mNAcc (AP: $1.54 \mathrm{~mm}$, ML: $0.7 \mathrm{~mm}$ ) with a Stereotaxic Drill (David Kopf Instruments). Dura was gently removed from cranial windows with fine surgical tweezers. A drop of $0.002 \%$ FG was placed on a piece of Parafilm (Bemis Company) and

manually backfilled into the prepared micropipette-Hamilton syringe set to the Stereotaxic Frame. The pipette was then lowered to the brain surface to reset the z-axis and slowly lowered to the site of infusion (INAcc, $4.5 \mathrm{~mm}$; mNAcc, $4.25 \mathrm{~mm}$ ). After a 10minute wait, FG was manually infused with a rate of approximately $50 \mathrm{~nL} / \mathrm{min}$, followed by another waiting period to ensure that the pipette had been emptied and FG diffused. The pipette was then gradually removed from the brain, and the protocol was repeated for infusion on the ipsilateral side before continuing with head-plate implantation.

\subsection{In vivo whole-cell patch-clamp recording}

In vivo patch-clamp method was carried out to record intrinsic activity from DA neurons using glass micropipette-electrodes. In this method, the pipette tip makes a tight seal with the neuronal membrane in the cell-attached configuration, and a small patch of the cell membrane at the pipette tip is subsequently broken by the application of suction to create the whole-cell configuration, which enables direct access to the neuron's subthreshold membrane activity in the intact brain and diffusion of neurobiotin (NB) into the cell (Fig. 3.3) (Noguchi et al., 2021). NB is a derivative of the vitamin biotin that is widely used for anterograde and retrograde neuronal labeling (Huang et al., 1992). Biotin is easily detected by the protein avidin that forms the avidin-biotin complex due to avidin's high affinity to biotin (Wilchek and Bayer, 1990). Recording electrodes were made with borosilicate glass capillaries (G120F-4, Warner Instruments) pulled with a pipette puller (DMZ Universal Electrode

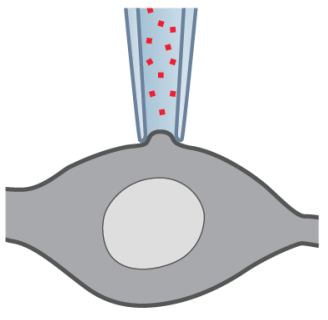

Cell-attached

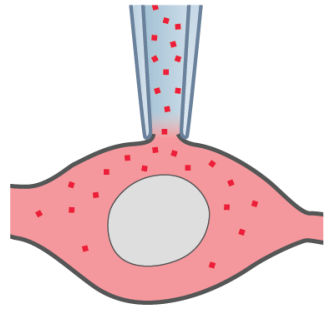

Whole-cell

Figure 3.3. In vivo patch-clamp configurations. 
Puller, Zeitz-Instruments) to have a tip resistance of 5 to $12 \mathrm{M} \Omega$. Pre-pull and last pull program was set as follows:

Table 3.1. Program parameters for the DMZ Universal Electrode Puller.

\begin{tabular}{|c|c||c|c|}
\hline \multicolumn{4}{|c|}{ Pre-pull P(A) } \\
\hline$H$ & 500 & $t(F 1)$ & 000 \\
\hline$F(T H)$ & 020 & $F 1$ & 000 \\
\hline$s(T H)$ & 100 & $s(F 2)$ & 000 \\
\hline$t(H)$ & 052 & $F 2$ & 000 \\
\hline$s(H)$ & 040 & $A D$ & 111 \\
\hline \multicolumn{2}{|c|}{ Last pull P(B) } \\
\hline$H$ & $300-600$ & $t(F 1)$ & 070 \\
\hline$F(T H)$ & 020 & $F 1$ & 060 \\
\hline$s(T H)$ & 008 & $s(F 2)$ & 004 \\
\hline$t(H)$ & 030 & $F 2$ & 070 \\
\hline$s(H)$ & 000 & $A D$ & 000 \\
\hline
\end{tabular}

$\mathrm{H}=$ heat, $\mathrm{F}(\mathrm{TH})=$ Force pre-pull, $\mathrm{s}(\mathrm{TH})=$ distance threshold, $\mathrm{t}(\mathrm{H})=$ delay heatstop, $\mathrm{s}(\mathrm{H})=$ distance heatstop, $\mathrm{t}(\mathrm{F} 1)=$ delay pull1, $\mathrm{F} 1=$ force pull1, $\mathrm{s}(\mathrm{F} 2)$ distance pull $2, \mathrm{~F} 2=$ force pull2, $\mathrm{AD}=$ adjust $/$ polishing

The heat setting of the last pull was adjusted each time to obtain the desired pipette resistance. Pre-made internal recording solution (Table 3.2) containing NB (Vector Laboratories) was stored at $-80{ }^{\circ} \mathrm{C}$ and defrosted prior to recording. The estimated free $\mathrm{Ca}^{2+}$ concentration was approximately $80 \mathrm{nM}$, calculated using an open-source program Maxchelator (https://somapp.ucdmc.ucdavis.edu/pharmacology/bers/maxchelator/) based on a $\mathrm{Ca}^{2+}$ contamination of $15 \mu \mathrm{M}$ (Bers et al., 2010; Woehler et al., 2014). Internal solution was filtered using a syringe filter (Whatman Anotop syringe filter $0.02 \mu \mathrm{m}$, Whatman) and kept in an ice bucket for the duration of recording.

Recordings were carried out at least 2 days after head-plate implantation, or 7-10 days after FG infusion. Experimental animals were placed in an isoflurane induction chamber and subsequently moved to the recording platform inside a Faraday cage. A mask was applied to the animals' face to maintain them under a constant flow of 
isoflurane $(1.0-2.5 \%$ in $100 \% 02,0.35 \mathrm{~L} / \mathrm{min})$. The head-plate attached to the animals was placed and screwed to the customized recording stage (Luigs \& Neumann). Breathing rate was kept at approximately $1 \mathrm{~Hz}$ and body temperature was maintained at $37-38{ }^{\circ} \mathrm{C}$ with a custom electronic heating blanket. Ophthalmic ointment was applied to the eyes whenever necessary. Dexamethasone (0.5 $\mu \mathrm{L} / \mathrm{g}$ body weight) was subcutaneously administered to the neck and lidocaine was applied to the skull prior to craniotomy. Craniotomies were carried out at the reference indentations above the VTA and SNc using a biopsy punch (1 $\mathrm{mm}$ diameter, Integra Miltex) and duratomy followed. Once the cranioduratomized area was clean, the pipette was filled with internal solution and placed into the pipette holder, which was then set to the head-stage connected to the patch-clamp amplifier (EPC10 USB, HEKA). The glass electrode was lowered to the brain surface with $\sim 1,000$ mbar positive pressure, at which point the micromanipulator (SM7, Luigs \& Neumann) was set to $\mathrm{z}=0$. The electrode was then quickly lowered down into the brain and the craniotomy site was covered with saline, in which a reference electrode was placed. Test pulses were continuously applied through the electrode in the voltageclamp mode. A tone generator (PSA-12, HEKA) combined with the oscilloscope of the PatchMaster software (HEKA) enabled continuous monitoring of the pipette resistance. Depending on the pipette resistance, positive pressure was adjusted to $600-1,000$ mbar. The electrode was then advanced to approximately $200 \mu \mathrm{m}$ above the midbrain, and the pressure was reduced and kept in the range of 40-70 mbar while probing for cells in the VTA (DV: $4.0-5.2 \mathrm{~mm}$ ) and SNc (DV: 3.8-5.2 mm). Fluctuations of the pipette resistance increased as the glass electrode approached a cell. Once the electrode was in good proximity of the cell, positive pressure was released to obtain a gigaohm seal and the voltage setting was lowered step-wise to -50 to $-60 \mathrm{mV}$. Cell-attached/on-cell activity was

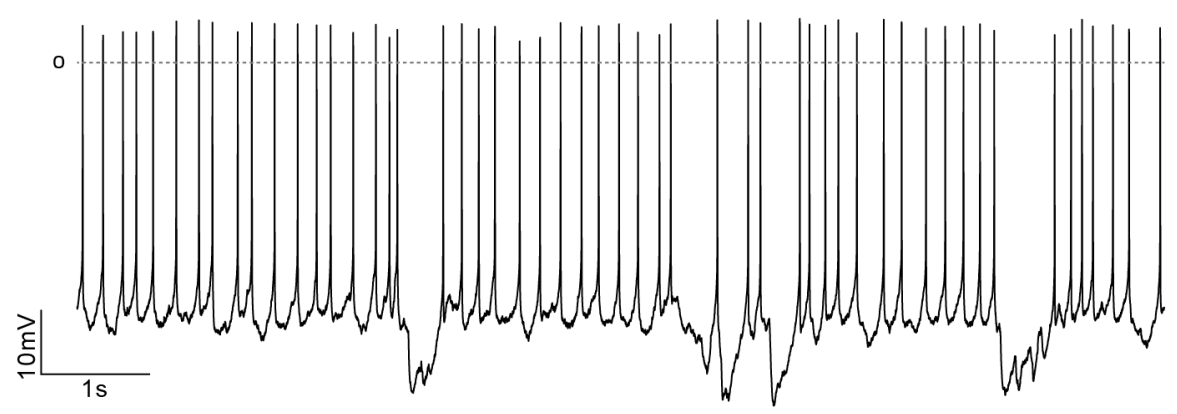

Figure 3.4. In vivo activity of dopamine neuron recorded in the whole-cell configuration. 
recorded for a subset of neurons in the voltage-clamp mode. A small suction was subsequently applied through tubing to break the cell membrane at the pipette tip. Upon successful entrance into the whole-cell mode, cell capacitance and series resistance were estimated. Recording of spontaneous activity (Fig. 3.4) and current injection protocols was obtained in the current-clamp mode. During current injection protocols, hyperpolarizing current of increasing amplitude was injected to the neuron in a step-wise manner (-5 to $-15 \mathrm{pA}$ steps), with 2-second injections at 8-second intervals, until the membrane potential reached $-80 \mathrm{mV}$. NB was diffused into the cell through the pipette during the length of recording. Data were acquired with PatchMaster at a sampling rate of $20 \mathrm{kHz}$. After the termination of recording, the recording electrode was slowly retracted until it was at least 200 to $300 \mu \mathrm{m}$ above the recorded neuron, then gradually withdrawn from the brain. Upon conclusion of the recording session, animal was sacrificed with a lethal injection of pentobarbital $(1.6 \mathrm{~g} / \mathrm{kg}$; Narcoren, Boehringer Ingelheim) and transcardially-perfused with a paraformaldehyde (PFA)-containing fixation agent (Table 3.2). Brain was carefully removed from the skull and kept in the fixative solution overnight, then stored in storing solution (Table 3.2).

Table 3.2. Summary of solutions used for experiments and their compositions.

\begin{tabular}{|c|c|}
\hline Solution & Composition \\
\hline $\begin{array}{l}\text { Internal recording } \\
\text { solution }\end{array}$ & $\begin{array}{c}135 \mathrm{mM} \text { K-gluconate, } 10 \mathrm{mM} \text { HEPES, } 5 \mathrm{mM} \mathrm{KCl}, 5 \mathrm{mM} \mathrm{MgCl} \\
\text {, }\end{array}$ \\
\hline $\begin{array}{l}\text { Extracellular } \\
\text { recording solution }\end{array}$ & $0.5 \mathrm{M} \mathrm{NaCl}, 10 \mathrm{mM}$ HEPES, $1.5 \% \mathrm{NB} ; \mathrm{pH}=7.4$ \\
\hline $\begin{array}{l}\text { Phosphate-buffered } \\
\text { saline (PBS), } 0.01 \mathrm{M}\end{array}$ & $\begin{array}{c}137 \mathrm{mM} \mathrm{NaCl}, 2.7 \mathrm{mM} \mathrm{KCl}^{10} \mathrm{mM} \mathrm{NaH}_{2} \mathrm{PO}_{4}, 10 \mathrm{mM} \mathrm{Na}_{2} \mathrm{HPO}_{4} ; \\
\mathrm{pH}=7.4\end{array}$ \\
\hline Fixation agent & $4 \%$ PFA, $15 \%$ picric acid, in $0.01 \mathrm{M} \mathrm{PBS} ; \mathrm{pH}=7.4$ \\
\hline Storing solution & $10 \%$ sucrose, $0.05 \% \mathrm{NaN} 3$, in $0.01 \mathrm{M}$ PBS; $\mathrm{pH}=7.4$ \\
\hline Blocking solution & $\begin{array}{c}10 \% \text { Horse serum, } 0.5 \% \text { Triton } \mathrm{X}-100,0.2 \% \text { Bovine serum } \\
\text { albumin (BSA), in } 0.01 \mathrm{M} \text { PBS; } \mathrm{pH}=7.4\end{array}$ \\
\hline Carrier solution & $\begin{array}{l}\text { 1\% Horse serum, } 0.5 \% \text { Triton X-100, 0.2\% BSA, in } 0.01 \text { M PBS; } \\
\text { pH }=7.4\end{array}$ \\
\hline
\end{tabular}




\subsection{In vivo extracellular recording and juxtacellular labeling}

Acute extracellular recording was carried out in a similar manner as in vivo patchclamp but on naïve animals (e.g. no prior surgery). Extracellular recording solution (Table 3.2) containing NB was made prior to recordings and stored at $4^{\circ} \mathrm{C}$. Glass electrodes were pulled to have a tip resistance of 10 to $23 \mathrm{M} \Omega$, using the same puller programs as above (Table 3.1). After initial isoflurane induction, animals were fixed with ear bars to a Stereotaxic Frame inside a Faraday cage and continuously anesthetized with isoflurane through a mask. Scalp incision and leveling were performed as described. Craniotomies were performed above the VTA (AP: -3.08 mm, ML: $\pm 0.25-0.75 \mathrm{~mm}$ ) or SNc (AP: -3.08 mm, ML: 0.9-1.4 mm) with a Stereotaxic Drill. A reference electrode was placed between the scalp and skull, behind the ear. Glass micropipette with recording solution was inserted into the electrode holder (Harvard Apparatus) and set to the Stereotaxic Frame. Once the microelectrode touched the brain surface, z-axis of the micromanipulator (SM-6, Luigs \& Neumann) was reset to zero, and the electrode was advanced to the midbrain (3.6-5.2 mm). Resistance of microelectrode was checked with square current test pulses (ELC-01MX, NPI Electronic) from time to time to maintain it within a recordable range. During probing of cells, electrical activity from the pipette was monitored via an oscilloscope (HM1008-2, HAMEG Instruments) as well as an audio signal (AUDIS-03/8M, NPI Electronic). Signals from single-unit activity were amplified $(1,000 \mathrm{x})$ and notch- and bandpass-filtered at $50 \mathrm{~Hz} \& 0.3-5,000 \mathrm{~Hz}$ through an electrodeclamp/amplifier/filter module complex (EXT-10-2F/ELC-01MX/DPA-2FS, NPI Electronic) and obtained at a sampling rate of $12.5 \mathrm{kHz}$ with the PatchMaster software via an analog-to-digital converter (EPC-10, HEKA). DA neuron identify was putatively determined by their slow firing frequencies (1-10 Hz) and broad biphasic action

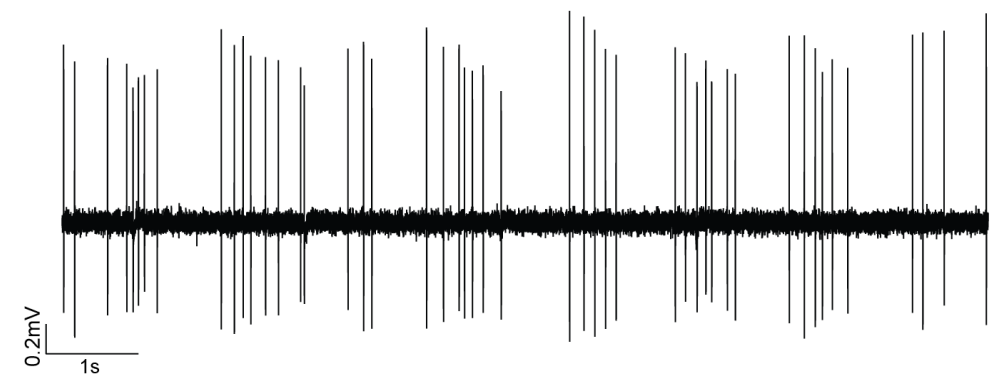

Figure 3.5. Spontaneous activity of extracellularly-recorded in vivo dopamine neuron. 
potentials (Ungless and Grace, 2012) measured on the oscilloscope. After acquiring at least 10 minutes of spontaneous activity (Fig. 3.5), juxtacellular labeling (Pinault, 1996) was carried out by delivering 200-ms on/off positive current pulses of up to $10 \mathrm{nA}$ with slowly-increasing intensity to induce internalization of NB. The electrode was carefully removed from the brain after iontophoretic labeling. Upon completion of recording session, animals were sacrificed and perfused for brain extraction as described.

\subsection{Immunohistochemistry}

Coronal sections of fixed brains (midbrain: $60 \mu \mathrm{m}$; striatum: $100 \mu \mathrm{m}$ ) were prepared with a vibrating microtome (VT1000 S, Leica Biosystems). The left hemisphere of each section was punched with a needle to ensure that left and right hemispheres could be distinguished. Prepared sections were then placed in a vial and rinsed 3 times (10 minutes per wash) in phosphate-buffered saline (PBS), followed by incubation in blocking solution (Table 3.2) for 1 hour in order to block unspecific binding sites and reduce background signals. Slices were then washed again in PBS 3 times and incubated overnight at room temperature (RT) in carrier solution (Table 3.2) containing primary antibodies (Table 3.3). Sections were subsequently washed in PBS 3 times and incubated in carrier solution (Table 3.2) with secondary antibodies (Table 3.4) at RT for at least 6 hours. Sections were then washed for the last time in PBS 3 times and mounted onto microscope slides in the proper anatomical (e.g. rostrocaudal) order. Once dried, mounted slices were covered with mounting medium (Vectashield, Vector Laboratories) followed by glass coverslips which were then secured with transparent nail polish to prevent mounting medium from drying. Microscopic slides were stored at $4^{\circ} \mathrm{C}$.

For staining midbrain sections containing NB-labeled neurons without retrograde tracing, either a combination of polyclonal rabbit anti-TH primary antibody, anti-rabbit 488 secondary antibody and 568 streptavidin conjugate; or monoclonal mouse anti-TH primary antibody, anti-mouse 488 secondary antibody and 568 streptavidin conjugate were used. For striatal sections containing FG infusion sites, either rabbit anti-TH primary and anti-rabbit 488 secondary antibodies, or mouse anti-TH primary and antimouse 488 secondary antibodies were used. No additional staining was carried out for FG in the striatum as the intensity of FG's intrinsic fluorescent signals was sufficient to 
identify infusion sites. For staining midbrain sections containing NB-labeled neurons and retrogradely-traveled FG, the following antibody combination was used: mouse anti-TH and rabbit anti-FG primary antibodies, anti-mouse 647 and anti-rabbit 568 secondary antibodies, and 488 streptavidin conjugate.

Table 3.3. List of primary antibodies used for immunohistochemistry.

\begin{tabular}{|c|c|c|c|c|}
\hline Primary Antibody & Class & Host & Dilution & Supplier \\
\hline \hline Anti-tyrosine hydroxylase & Polyclonal & Rabbit & $1: 1000$ & Calbiochem \\
\hline Anti-tyrosine hydroxylase & Monoclonal & Mouse & $1: 1000$ & Millipore \\
\hline Anti-Fluoro-Gold & Polyclonal & Rabbit & $1: 1000$ & Fluorochrome \\
\hline
\end{tabular}

Table 3.4. List of secondary antibodies and conjugates used for immunohistochemistry.

\begin{tabular}{|c|c|c|c|}
\hline Secondary Antibody/Conjugate & Host & Dilution & Supplier \\
\hline \hline AlexaFluor 488 anti-rabbit & Goat & $1: 750$ & Invitrogen \\
\hline AlexaFluor 488 anti-mouse & Goat & $1: 750$ & Invitrogen \\
\hline AlexaFluor 488 streptavidin & & $1: 1000$ & Invitrogen \\
\hline AlexaFluor 568 anti-rabbit & Goat & $1: 750$ & Invitrogen \\
\hline AlexaFluor 568 streptavidin & & $1: 1000$ & Invitrogen \\
\hline AlexaFluor 647 anti-mouse & Goat & $1: 750$ & Invitrogen \\
\hline
\end{tabular}

\subsection{Imaging with laser scanning confocal microscope}

Fluorescent signals from immunolabeled sections containing recorded neurons were examined under a laser scanning confocal microscope (LSCM; Eclipse 90i, Nikon) and images were captured with Nikon C2Plus camera and exported via NIS-Elements software (Nikon). LSCM allows increased optical resolution and contrast of images by focusing the laser illumination to a small point in the specimen and by utilizing a pinhole to allow fluorescent signals from the desired focal plane to be detected while blocking out-of-focus signals from focal planes outside of interest (Jonkman and Brown, 2015). Confocal images of midbrain sections containing neurons co-labeled with NB and TH 
were acquired at low, medium, and high magnifications using 4x, 10/20x, and 60x (oil immersion) objective lenses, respectively. High magnification images were used to confirm co-localization of NB and TH, or NB, TH and FG. Low and medium magnification images were necessary to determine the anatomical location of labeled neurons. To see morphological features of recorded neurons, 60x Z-stack images were acquired at 1- $\mu \mathrm{m}$ intervals, which were processed with the Maximal Intensity Projection algorithm on NISElements. Additionally, FG-infused striatal sections were imaged with a 4x objective lens to confirm the infusion sites.

\subsection{Data extraction and quantification}

Raw recording traces acquired with PatchMaster were exported to MATLAB (MathWorks) for quantification and analysis. All activity-related values were extracted in relation to time. For cell-attached recordings, the first derivate $(d V / d t)$ of recording traces was obtained and threshold of spike detection was manually determined for each trace, where every point crossing over the threshold of detection upward was detected as a spike. Spike detection of extracellular recordings was performed in a similar fashion by manually setting the threshold of spike detection. Mean firing rate (FR) was calculated as the total number of spikes divided by the length of recording. Interspike interval (ISI) was calculated as an interval between two spikes. Mean coefficient of variation (CV) was calculated as standard deviation (SD) of ISI divided by mean ISI, multiplied by 100 .

For spontaneous current-clamp recordings, spike thresholds $\left(\mathrm{V}_{\mathrm{thr}}\right)$ were first extracted based on $\mathrm{dV} / \mathrm{dt}$ of recording traces, followed by action potential peaks, membrane potential minima $\left(\mathrm{V}_{\mathrm{min}}\right)$, ISI, $\mathrm{CV}$, spike half-widths, and spikes fired as bursts (SFB), when applicable. Spikes were detected if the following two conditions were met: 1) $\mathrm{dV} / \mathrm{dt}$ of recording trace reaches $5 \mathrm{mV} / \mathrm{ms}\left(=V_{\mathrm{thr}}\right)$, and 2) $\mathrm{dV} / \mathrm{dt}$ of recording trace reaches $-2 \mathrm{mV} / \mathrm{ms}$ within $3 \mathrm{~ms}$ of (1) (Fig. 3.6). The parameter for spike detection may vary in different studies examining distinct types of neurons (Naundorf et al., 2006; Yu et al., 2008). $\mathrm{dV} / \mathrm{dt}=5 \mathrm{mV} / \mathrm{ms}$ was chosen as it reliably extracted spikes in my dataset. While $\mathrm{dV} / \mathrm{dt}=10 \mathrm{mV} / \mathrm{ms}$ is generally used for spike detection of in vitro current-clamp recordings of midbrain DA neurons, this parameter was not optimal for in vivo recordings, in particular for burst spikes in which the rate of depolarization decreased 

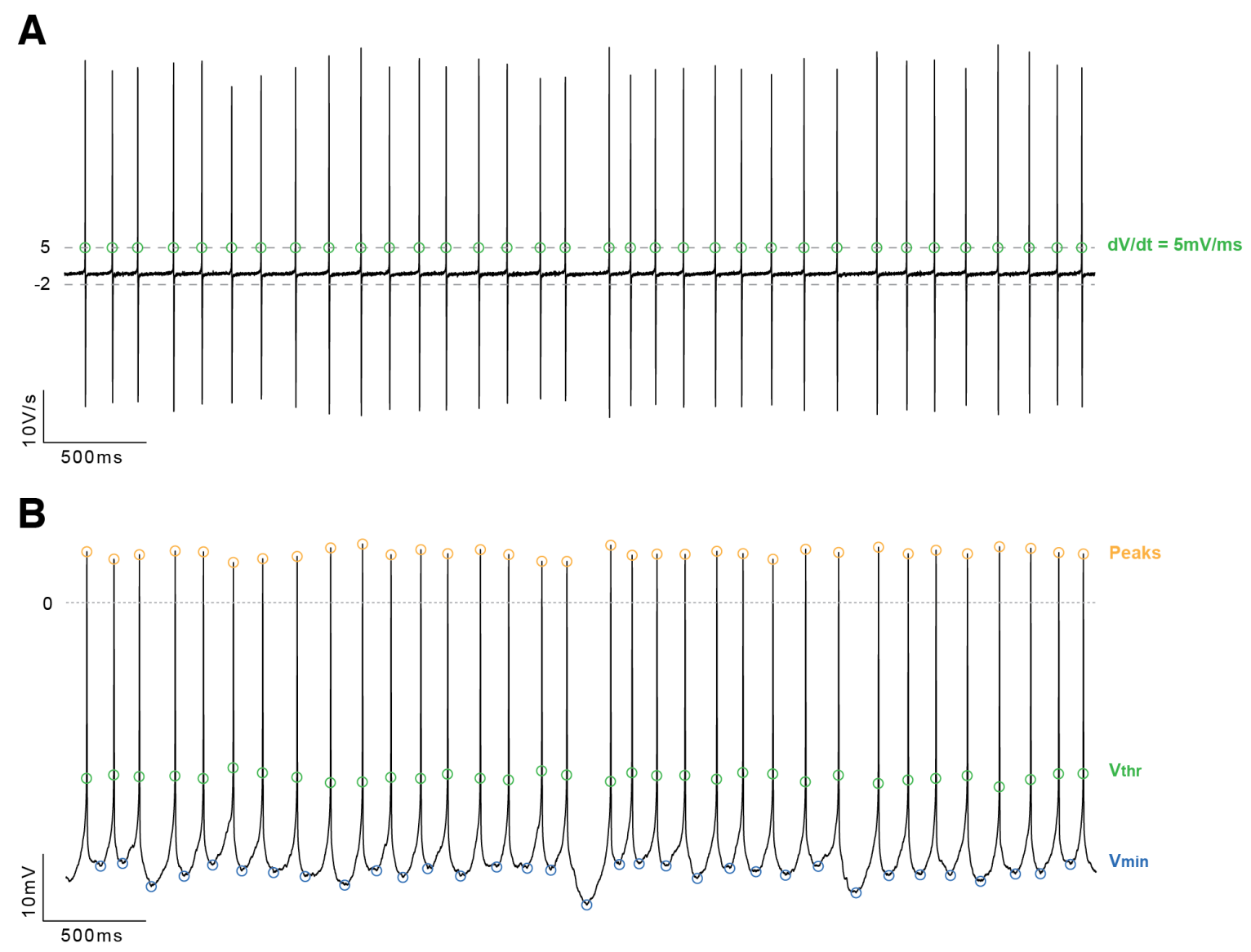

Figure 3.6. First derivative-based spike detection of a pacemaker neuron. A) First derivative of a recording trace showing $\mathrm{V}_{\mathrm{thr}}$, where two criteria met (green circles; $\mathrm{dV} / \mathrm{dt} \geq$ $5 \mathrm{mV} / \mathrm{ms} \& \mathrm{dV} / \mathrm{dt} \leq-2 \mathrm{mV} / \mathrm{ms}$ within $3 \mathrm{~ms})$. B) The actual recording trace in which peaks and $V_{\min }$ were detected based on $V_{\text {thr }}$.

with each spike (Fig. 3.7). In some cases, additional criteria such as maximal or minimal peak values were added to eliminate artifacts and optimize the performance of spike detection. Maximal voltage value of the trace that occurs within $3 \mathrm{~ms}$ of $\mathrm{V}_{\text {thr }}$ was taken as peak amplitude, and minimal voltage value between two adjacent peaks was taken as $V_{\min }$. One exception was made for the extraction of spike half-widths (e.g. spike widths at half-maximal spike amplitude), for which $\mathrm{dV} / \mathrm{dt}=10 \mathrm{mV} / \mathrm{ms}$ was used instead of 5 $\mathrm{mV} / \mathrm{ms}$ for easier comparison with in vitro data. Half-widths instead of full-widths were chosen due to the kinetics of afterhyperpolarization (AHP) in some spikes of in vivo DA neurons (Fig. 3.8).

Sag amplitude and rebound delay values were extracted from recordings that underwent current injection protocols, during which membrane potentials had reached $-80 \mathrm{mV}$. Sag amplitude was calculated as the voltage difference between the most 
A

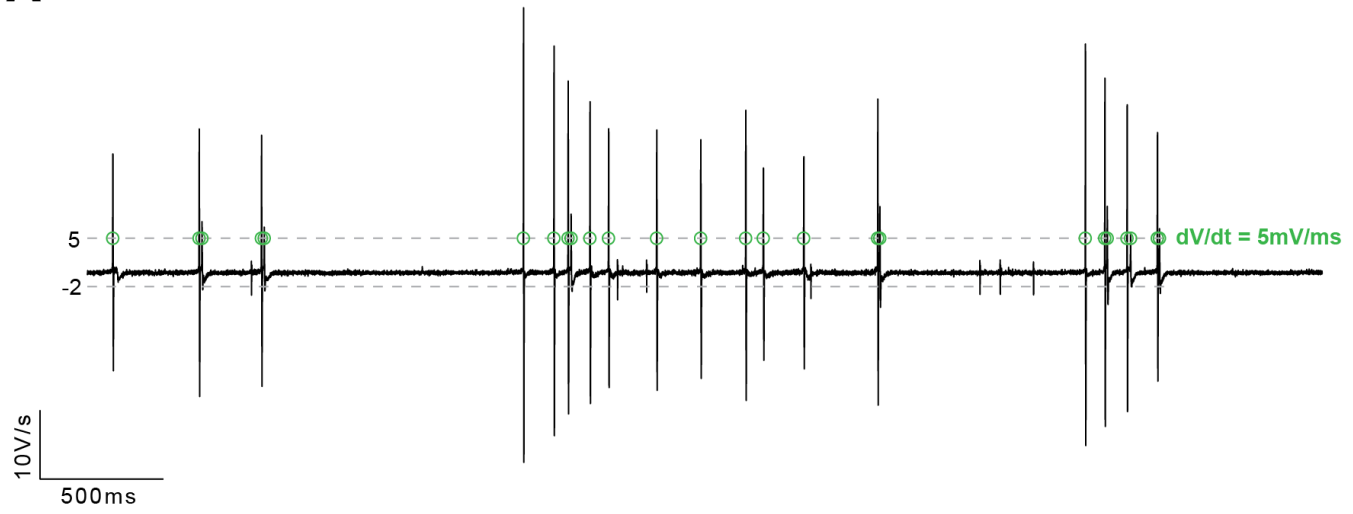

B

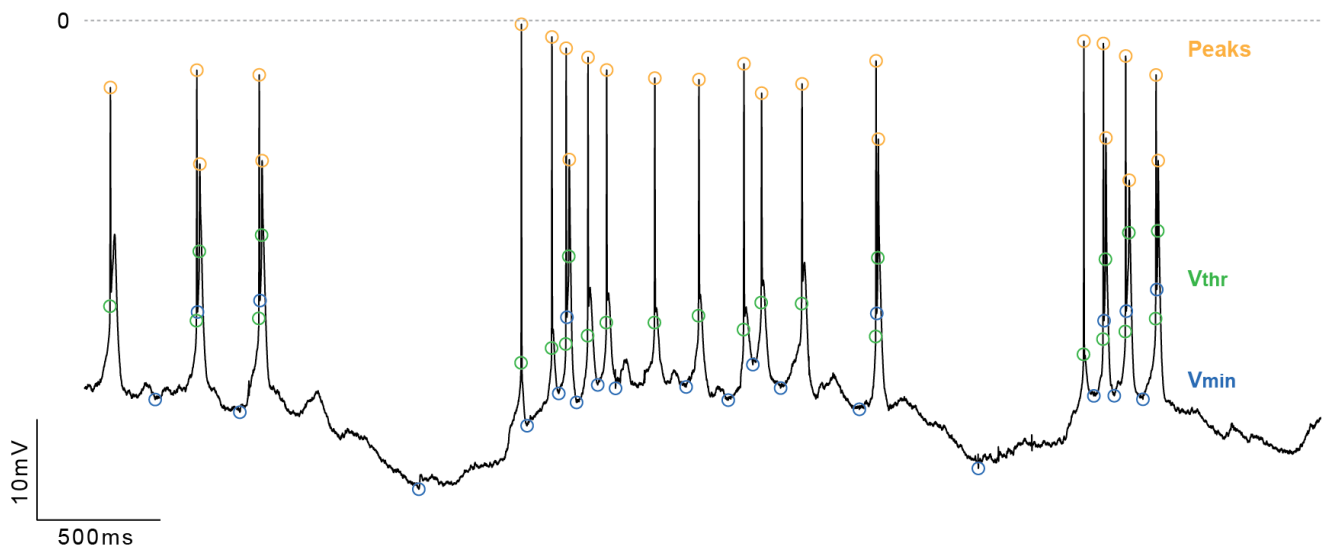

Figure 3.7. First derivative-based spike detection of a rebound bursting neuron. A) First derivative of an example recording trace showing $\mathrm{V}_{\mathrm{thr}} . \mathrm{dV} / \mathrm{dt}>10 \mathrm{mV} / \mathrm{ms}$ does not detect burst patterns in dopamine neurons due to the decreasing rate of depolarization. B) The same recording trace in which peaks and $V_{\min }$ were detected based on $V_{\text {thr }}$. Spikelets are not detected as spikes.

hyperpolarized membrane potential towards the start of injection (approximately -80 $\mathrm{mV}$ ) and the averaged membrane potentials of the last $100 \mathrm{~ms}$ during injection (Fig. 3.9A, sag amplitude $=a$ ). Rebound delay was calculated as the time difference between the termination of injection to the first spike following injection (Fig. 3.9B, rebound delay = b). Input resistance was calculated using Ohm's law; the voltage difference between the membrane potential immediately prior to injection and the most hyperpolarized membrane potential, divided by the injected current (Fig. 3.9B, input resistance $=c / d$ ).

Rebound oscillations were computed from 9 neurons that exhibited two clearlysegregated ISI- $\mathrm{V}_{\min }$ clusters. Inter-rebound intervals were calculated for time-points of all events residing in ISI- $\mathrm{V}_{\min }$ Cluster 1 which were then converted to Hertz. 


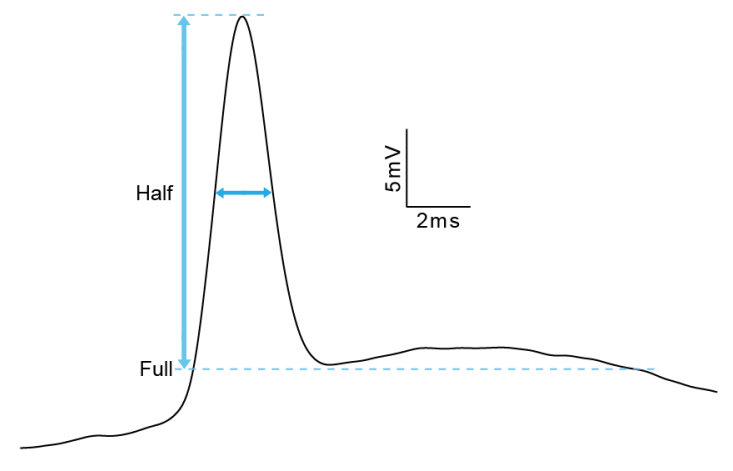

Figure 3.8. Quantification of spike half-widths. Half-widths (spike widths at half-maximal spike amplitude) were calculated instead of the full-widths due to the kinetics of afterhyperpolarization in some spikes as shown.

A

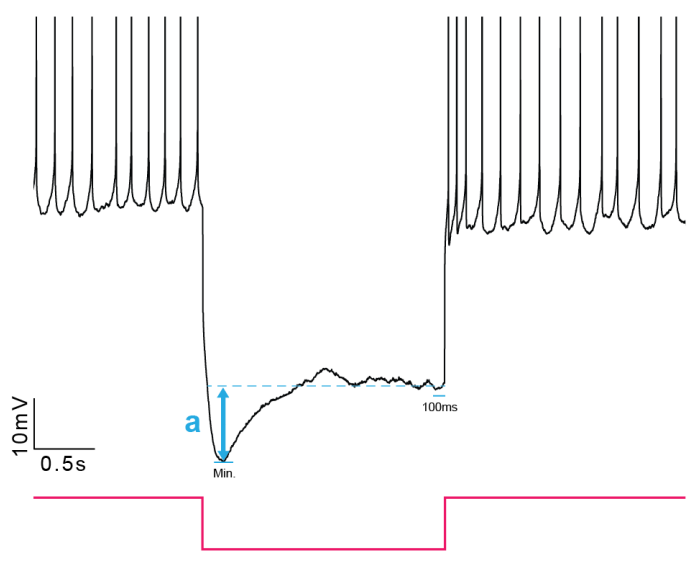

B

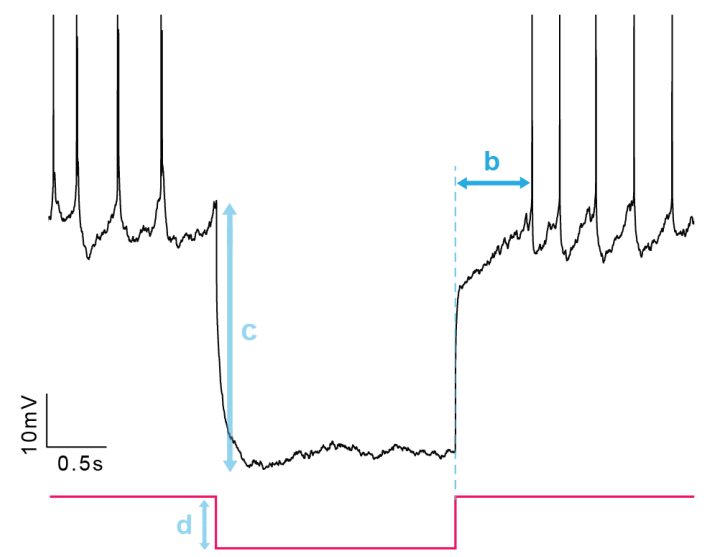

Figure 3.9. Quantification of sag amplitude, rebound delay, and input resistance. Recording traces from 2-second negative current injection (pink) protocols. A) Sag amplitude is denoted here as $a$. B) Rebound delay is denoted here as $b$. Input resistance was calculated as $c / d$.

\subsection{Plots and analyses}

All maps and plots were created with custom MATLAB scripts, apart from onedimensional plots, which were created on Prism 8 (GraphPad). Normality tests, Wilcoxon signed-rank tests, Mann-Whitney U tests, and t-tests were carried out on Prism 8.

Anatomical maps and anatomical heat maps were created by first manually inputting each location of confirmed DA neurons based on low-magnification confocal 
images onto a preset coronal midbrain image (2000 x 1500 pixels) that corresponded to their rostrocaudal plane (Paxinos and Franklin, 2007). Registered locations were saved as xy-coordinates and subsequently concatenated with FR, CV, sag amplitude, and rebound delay values to create heat maps with a coronal midbrain image as the background.

Gaussian curve fitting was performed using the fitdist and fitgmdist functions on MATLAB. ISI- $V_{\min }$ and ISI- $V_{\text {thr }}$ clusters in scatterplots were identified by Euclidean distance-based hierarchical clustering. For event-triggered averaging plot of pacemakers, 10 non-overlapping spikes were superimposed and aligned at the $V_{\text {thr }}$ time-point then averaged to show stereotypical behavior surrounding pacemaking spikes. For rebound bursters, 10 random spikes preceded by ISI- $V_{\min }$ Cluster 1 events were superimposed and aligned at the time of each $V_{\text {thr }}$ and averaged, while 10 spikes from ISI- $V_{\text {thr }}$ Cluster 2 events were used for plateau bursters.

Bimodality coefficients (BC) were calculated for $V_{\min }$ and $V_{\text {thr }}$ using the following equation:

$$
B C=\frac{m_{3}^{2}+1}{m_{4}+3 \frac{(n-1)^{2}}{(n-2)(n-3)}}
$$

where $m_{3}=($ skewness of sample $)$, and $m_{4}=($ kurtosis of sample $)-3$. The benchmark value of bimodality detection is $>0.555(=5 / 9)$ (Pfister et al., 2013).

As an alternative method of bimodality detection, Kernel density estimations (KDE) of $\mathrm{V}_{\min }$ and $\mathrm{V}_{\text {thr }}$ were first computed using the ksdensity function of MATLAB (Botev et al., 2010). Subsequently, first derivative was obtained from normalized KDE (Fig. 3.10B) to generate density derivative estimations (Chacón and Duong, 2020), and any point that crossed the threshold of $y=0$ on a downward slope of the density derivative curve was detected as the peak of a mode (Fig. 3.10C, blue circles). Extreme ends on the x-axis representing 1-2 events were excluded from analysis (15 units on both ends; Fig. 3.10C, gray zone). Adjacent peaks within 20 time-point units from each other in the derivative curve with similar peak values $(<0.4)$ in normalized KDE were considered to represent one mode, which was observed in irregular, broadly-spread $V_{\text {min }}$ and $\mathrm{V}_{\text {thr. }}$ Bandwidth of KDE is an important parameter that determines the extent of 
A

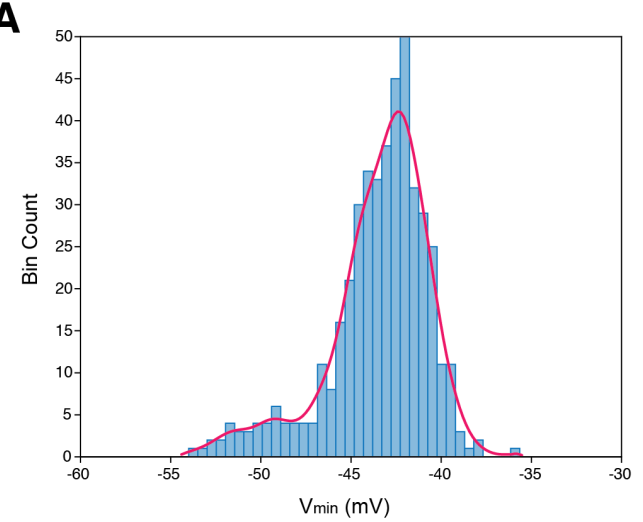

C

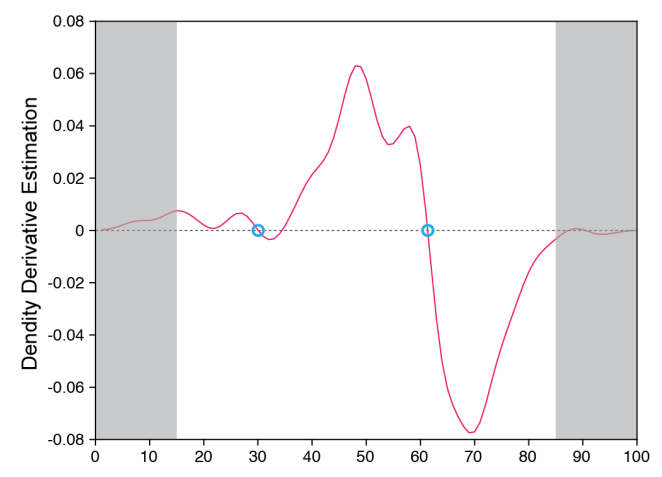

B

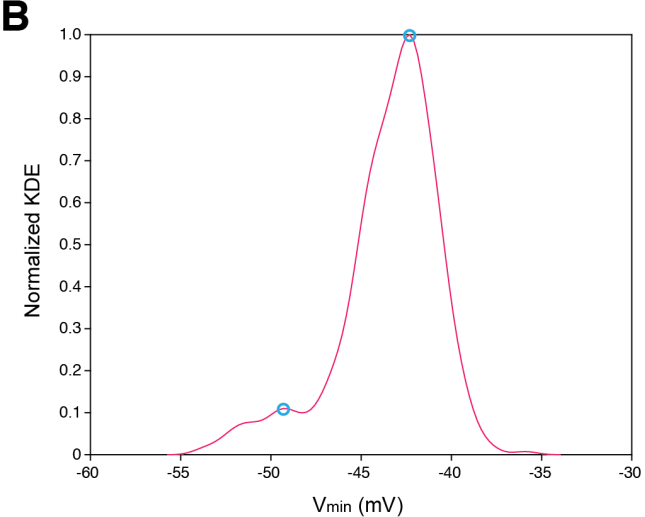

D

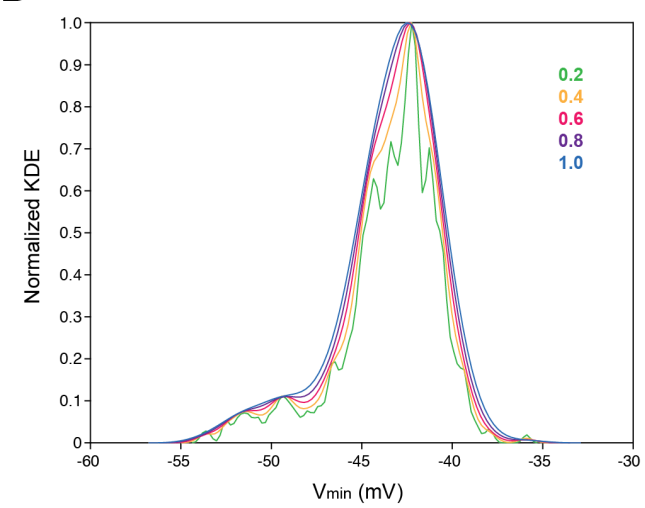

Figure 3.10. Modality detection by density derivative estimation. A) $A V_{\min }$ histogram and kernel density estimation (KDE; pink curve) with bandwidth of 0.65. B) Normalized KDE. C) First derivative of (B) for detection of mode peaks ( $\mathrm{dV} / \mathrm{dt}=0$; blue circles). Gray zones are excluded from analysis. D) Plots of KDE with different bandwidths $(0.2,0.4,0.6,0.8$ and 1.0$)$.

smoothing of the estimated curve. Upon manually examining bandwidths from 0.2 to 1.0 at 0.1 intervals and from 0.6 to 0.7 at smaller intervals for my dataset (Fig. 3.10D), the optimal bandwidth was determined to be 0.65 . Modality detection results obtained using this bandwidth value were tested against those from two different automatic bandwidth selectors on MATLAB (Botev, 2015) and the manually-determined value of 0.65 turned out to be a better performer. Recorded DA neurons were classified by their subthresholdactivity based firing patterns based on the number of detected modes in KDE, as follows: 
Table 3.5. Number of $\mathrm{V}_{\min }$ and $\mathrm{V}_{\mathrm{thr}}$ modes by subthreshold membrane activity-based firing pattern.

\begin{tabular}{|c|c|c|}
\hline Class & \# of $\mathbf{V}_{\text {min }}$ modes & \# of $\mathbf{V}_{\text {thr }}$ modes \\
\hline \hline Single-spike & 1 & 1 \\
\hline Rebound burst & 2 & 1 \\
\hline Plateau burst & $1-2$ & 2 \\
\hline Rebound-plateau burst & 3 & 2 \\
\hline
\end{tabular}




\section{RESULTS}

Exploring the subthreshold membrane potentials of midbrain dopamine neurons in vivo

\subsection{Establishing the deep-brain whole-cell patch-clamp recording method}

Phasic firing of DA neurons is an essential component of DA signaling that encodes a diverse range of behaviorally-relevant information. While the intrinsic mechanisms of DA tonic firing have been described in studies that used in vitro patch-clamp, those of phasic firing are not well understood as the presence of synaptic inputs is necessary for DA neurons to fire in bursts. In an attempt to fill this gap in our knowledge, in vivo wholecell patch-clamp recording technique was established to record subthreshold membrane activity of midbrain DA neurons in the intact brain. While this method has so far not been used on DA neurons, it is used to record from neurons in more superficial brain regions such as the cortex and hippocampus -either with visual guides or blindly- but also in deeper regions such as the thalamus and cerebellum (Tao et al., 2015). I used the blind patch-clamp method assisted with an oscilloscope and a tone generator, which enabled both visual and auditory monitoring of real-time resistance change at the pipette tip. In vitro patch-clamp method normally requires recording pipettes with resistance in the range of 2 to $5 \mathrm{M} \Omega$, depending on the type of experiments performed. For deep-brain in vivo patch-clamp, thinner glass capillaries $(1.2 \mathrm{~mm})$ used for in vivo extracellular recording were modified to have pipette resistance in the range of 6 to $10 \mathrm{M} \Omega$, which was found to be optimal for in vivo patch-clamping. This minimized the amount of recording solution with neurobiotin (NB) leaking into the brain while high positive pressure was being applied through the pipette, and also made it easier to achieve a gigaohm seal at deeper brain regions. Internal solution was modified for in vivo patch-clamp recording to achieve a more physiological free $\mathrm{Ca}^{2+}$ concentration of approximately $80 \mathrm{nM}$.

Experimental animals (adult male C57BL6/N, $\mathrm{N}=60 ; \mathrm{C} 57 \mathrm{BL6} / \mathrm{J}, \mathrm{N}=2$ ) had been implanted with a head-plate, which was used to firmly place them onto the recording platform, and were continuously anesthetized with isoflurane throughout the duration of recording (Fig. 4.1A, left). After performing a cranioduratomy, the recording pipette was lowered down from the cranial window with a high positive pressure of up to 1,000 mbar, 
A

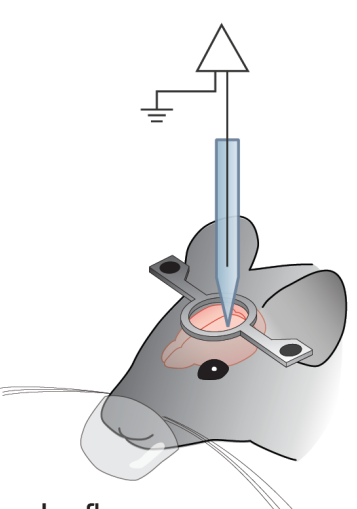

Isoflurane
C

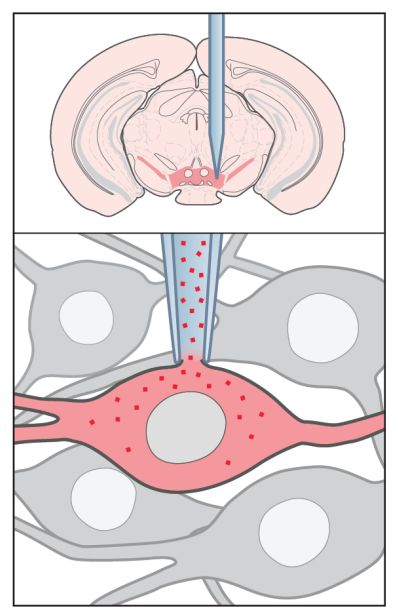

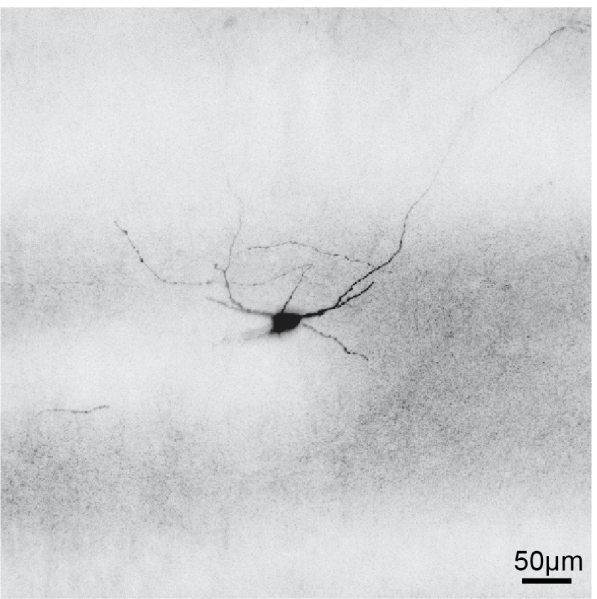

B
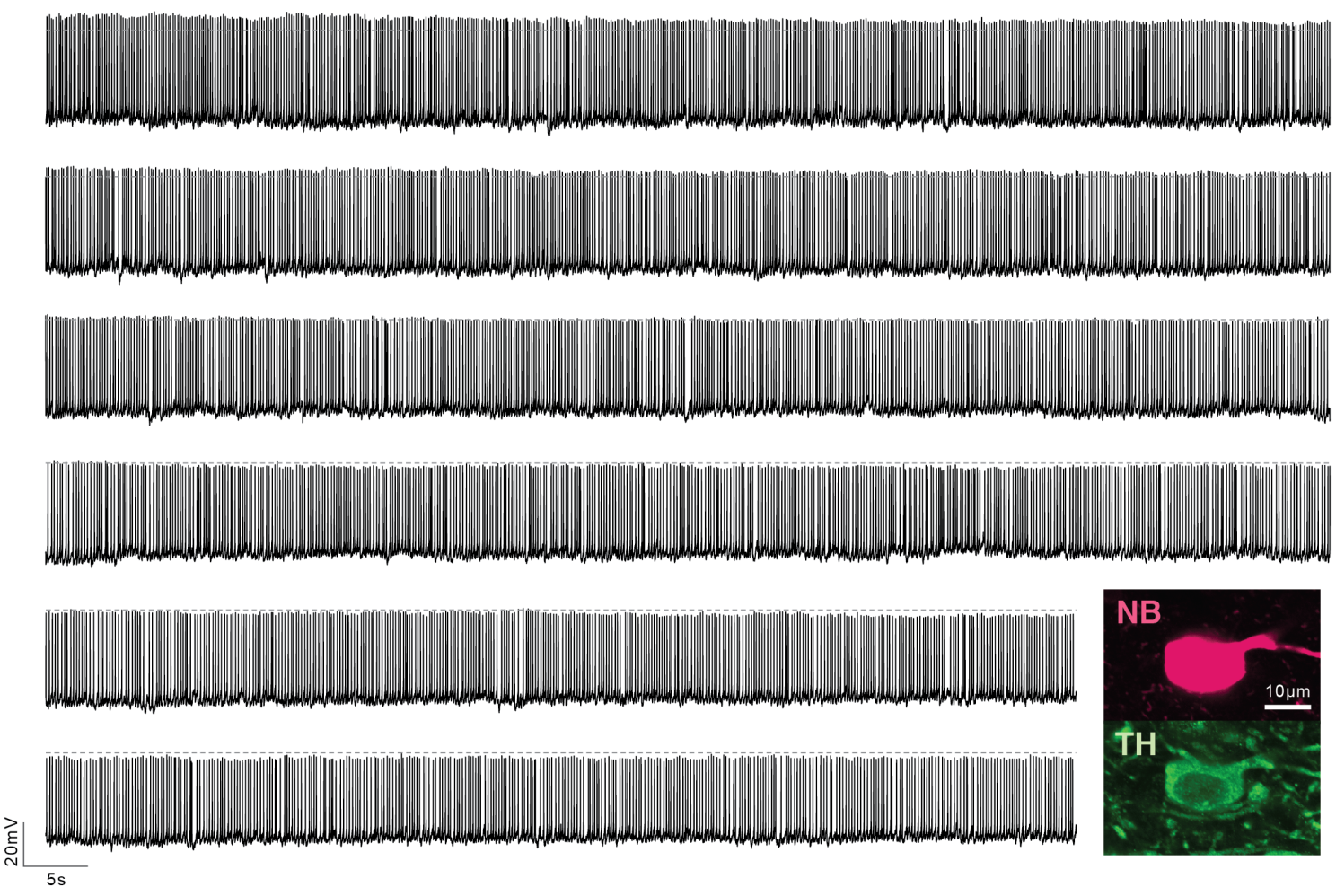

Figure 4.1. Establishing the in vivo patch-clamp method for midbrain dopamine neurons. A) Schematic of experimental design. Left: During recording, animals were anesthetized with isoflurane and head-fixed to the recording platform with a head-plate. Top right: Recording pipette was lowered down to the midbrain (red). Bottom right: Putative dopamine neurons were recorded in the wholecell configuration which allowed NB (red dot) to fill the neuron. B) Example long recording (9 min. 20 sec.) demonstrates stable activity of in vivo patch-clamped dopamine neuron. NB and TH were not washed out during long recordings. C) Z-stacked confocal image of recorded dopamine neuron shows NB filling in proximal and distal dendrites. NB: neurobiotin; TH: tyrosine hydroxylase. Brain illustration adapted with permission from G. Paxinos and K. Franklin, 2007. Copyright (2007) Elsevier. 
which was adjusted according to pipette resistance, until reaching approximately $200 \mu \mathrm{m}$ above the recording site. Pressure was then gradually reduced to 30 to 60 mbar while advancing into the target site (Fig. 4.1A, top right). The tone generator was crucial for blind probing of cells, as dynamic, up-and-down fluctuations of pipette resistance could be continuously monitored and more precisely evaluated based on the pitch, as opposed to the oscilloscope from which precise nature of fluctuations was difficult to obtain due to temporal limitations. As the pipette approached a neuron, pipette resistance and its fluctuations gradually increased. Based on my experience, a gigaohm seal can be most successfully achieved when resistance fluctuations are in 13 to $22 \mathrm{M} \Omega$ ranges, at which point pressure is released. If successful, pipette resistance instantly surpassed a G $\Omega$, or in less successful cases, reached a few hundred $M \Omega$, at which point an application of negative pressure and/or a few micrometer adjustments to the pipette's dorso-ventral coordinate was carried out to improve the seal. Once a stable gigaseal was achieved, it was possible to putatively determine its identity as DAergic or non-DAergic based on wide, narrow, or no waveform exhibited in response to test pulses. In this cell-attached mode, firing activity was recorded for a subset of putative DA neurons at a holding potential of -50 to $-60 \mathrm{mV}$. A small suction was then applied to break the membrane to achieve the whole-cell configuration to record spontaneous activity and response to hyperpolarization in the current-clamp mode, while NB filled the neuron by diffusion (Fig. 4.1A, bottom right). Series resistance was high and had a median of $63.58 \mathrm{M} \Omega$ (range = 46.39-124.60 M $\Omega$ ), which was likely due to the small pipette tip and deep recording depth that increased the chance of pipette contamination (Margrie et al., 2002). Other than a few instances in which the membrane gradually or abruptly resealed, recordings were stable over time and could be maintained for up to 30 minutes (Fig. 4.1B). Upon termination of recording, the pipette was slowly and carefully retracted to 200 to $300 \mu \mathrm{m}$ above the recording site, which normally took up to 30 minutes. The pipette was then gradually removed from the brain with increasing speed. Whenever possible, multiple recordings were obtained from both hemispheres (range $=1-7$ ). Animals were sacrificed immediately after completion of experiments and transcardially perfused with paraformaldehyde (PFA) for post hoc immunohistochemistry with tyrosine hydroxylase (TH) antibody and streptavidin for NB. Confocal imaging of immunostained tissues 
showed that recording for longer durations did not lead to washout of TH while allowing NB to fill dendrites (Fig. 4.1C). With this in vivo patch-clamp method, I was able to record from 141 putative DA neurons, 91 of which were successfully recovered and identified as midbrain DA neurons with post hoc immunohistochemistry ( $\mathrm{n}=91, \mathrm{~N}=62$ ) (Fig. 4.2A to $4.2 \mathrm{~F}$ ). Only the recordings whose top $20 \%$ of spikes had a mean threshold-to-peak amplitude greater than $20 \mathrm{mV}$ were included for subsequent electrophysiological analyses.
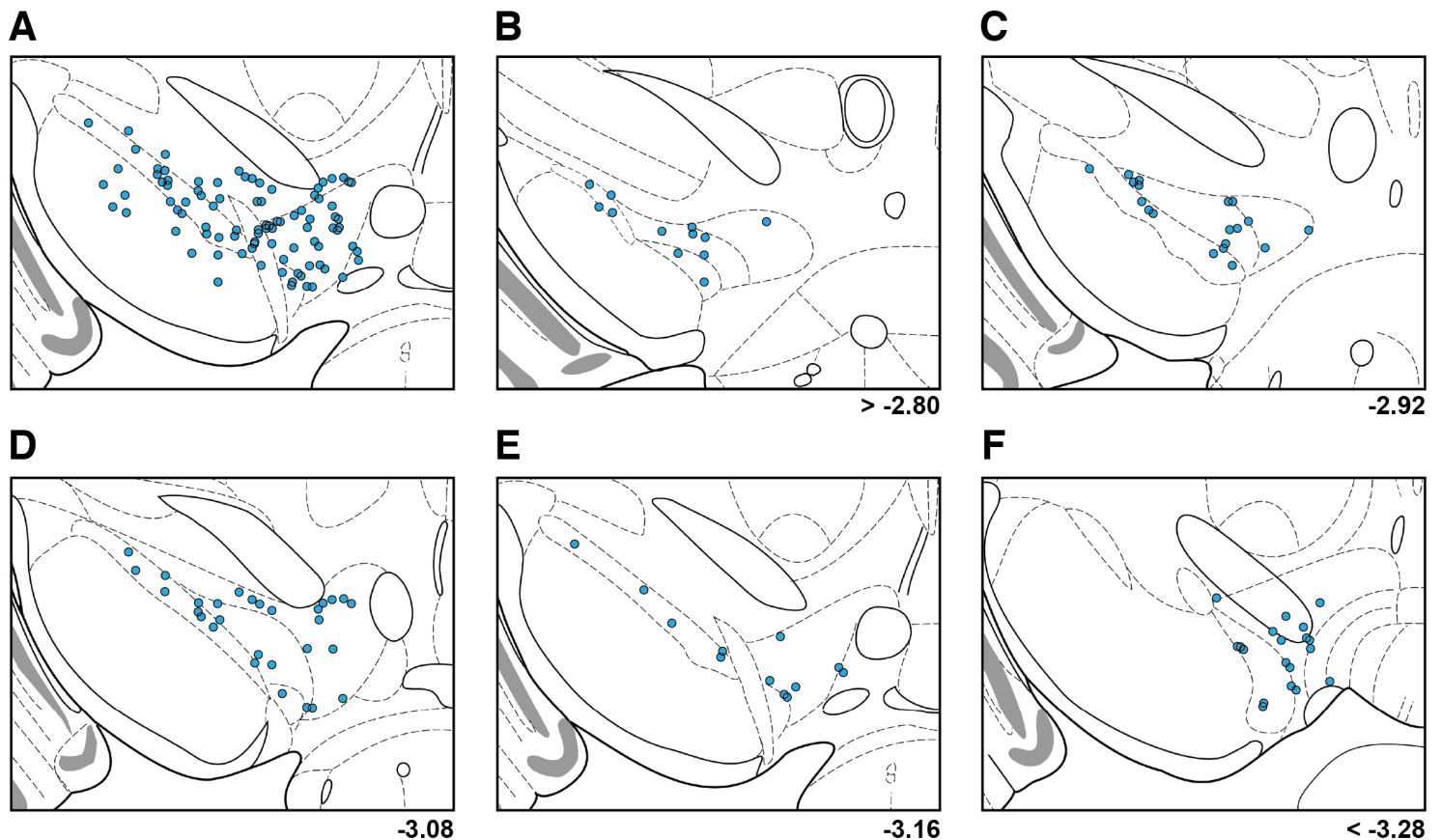

Figure 4.2. Maps illustrating the anatomical locations of 91 neurons recorded and identified as midbrain dopamine neurons with post hoc immunohistochemistry. A) Anatomical distribution of all recorded and identified dopamine neurons. Recorded dopamine neurons located rostral to Bregma = $-2.80 \mathrm{~mm}(\mathrm{~B})$, Bregma $\approx-2.92 \mathrm{~mm}(\mathrm{C})$, Bregma $\approx-3.08 \mathrm{~mm}(\mathrm{D})$, Bregma $\approx-3.16 \mathrm{~mm}(\mathrm{E})$, and caudal to Bregma $\approx-3.28(F)$. Brain illustrations adapted with permission from G. Paxinos and K. Franklin, 2007. Copyright (2007) Elsevier.

In establishing this method, it was crucial to verify that it worked as intended and that it did not disrupt the physiological processes of the neuron that might result in altered firing. Thus, in order to determine the viability of my in vivo DA neuron wholecell recordings, I first compared on-cell (cell-attached) activity prior to break-in with spontaneous activity from single-unit extracellular recordings. Extracellular recordings were obtained from histochemically-identified DA neurons in a separate set of experiments. Figure 4.3A shows on-cell activity (top) and whole-cell activity (bottom) 
from the same neuron. An example extracellular recording trace is shown in Figure 4.3B. Both on-cell firing rate (FR) and coefficient of variation (CV) of interspike intervals (ISIs) were comparable and matched the range of firing rate and $\mathrm{CV}$ from extracellular recordings (on-cell FR median(interquartile range, IQR) $=4.40$ (3.14-6.45) Hz, extracellular FR $=3.37(2.47-5.32) \mathrm{Hz}, \mathrm{p}=0.0741$, Mann-Whitney U test; on-cell CV median $(\mathrm{IQR})=45.84(31.76-78.70) \%$, extracellular $\mathrm{CV}=43.82(29.13-78.47) \%, \mathrm{p}=$ 0.769, Mann-Whitney test; on-cell $n=30$, extracellular $n=31$; Fig. 4.3C and 4.3D). Next,

A

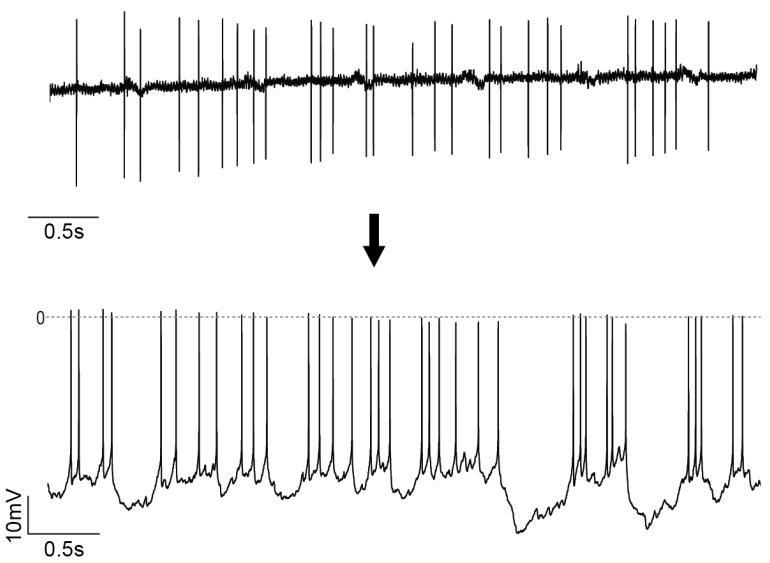

B

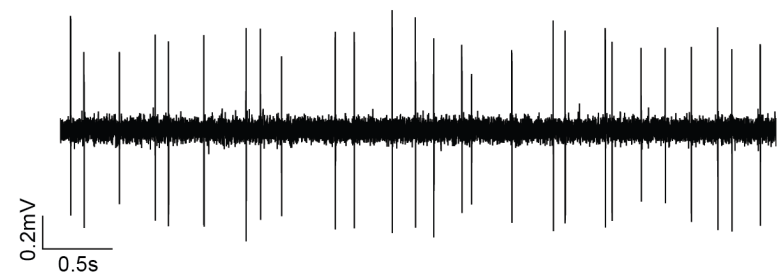

C

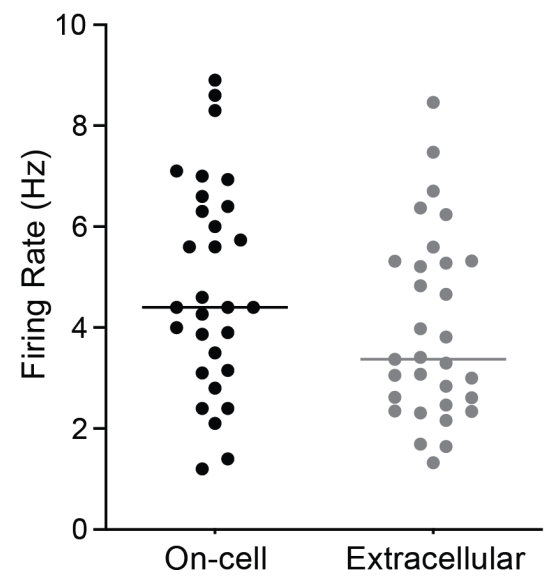

D

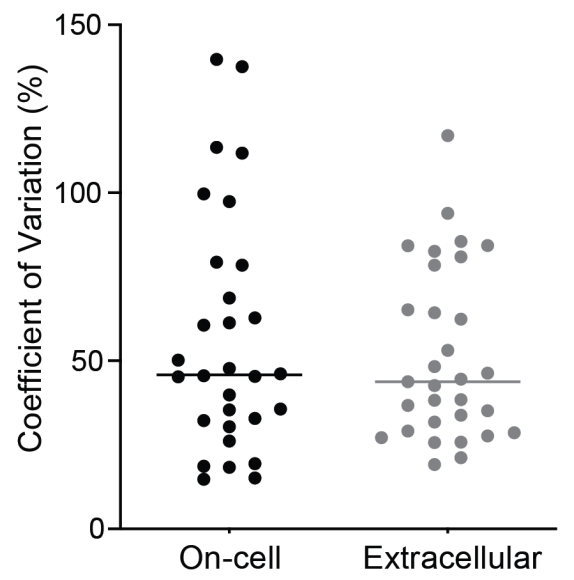

Figure 4.3. Comparisons with cell-attached and extracellular recordings demonstrate the viability of whole-cell recordings. A) Example traces of on-cell (top) and whole-cell (bottom) recording from the same dopamine neuron. B) Example single-unit extracellular activity from an in vivo dopamine neuron. C) Plot comparing mean firing rate of on-cell and extracelluar recordings shows no clear difference between the two groups apart from slight differences in the median. D) Plot comparing coefficient of variation in the two groups also shows no significant difference. 
to determine the stability of spontaneous activity of in vivo whole-cell recording over time, mean firing rate and CV were plotted against time at 10 -second intervals. Figure 4.4A shows that in many recordings, firing rate was higher immediately after break-in, and mostly stabilized after 50 seconds. In comparison, CV appeared relatively stable throughout recording in most neurons, although some neurons showed a slight, gradual

A

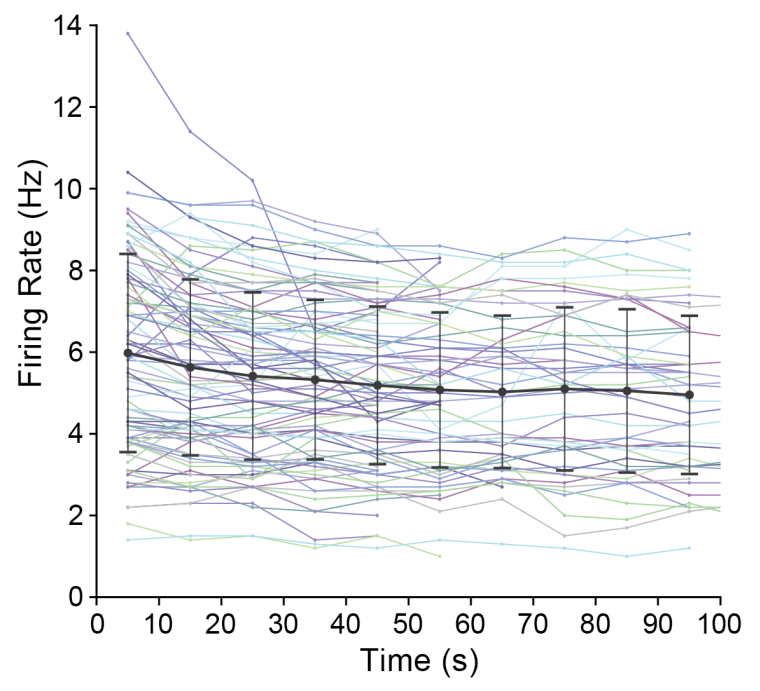

C

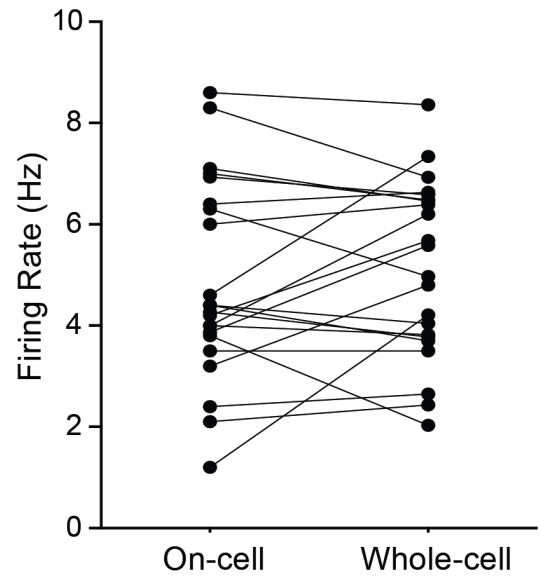

B

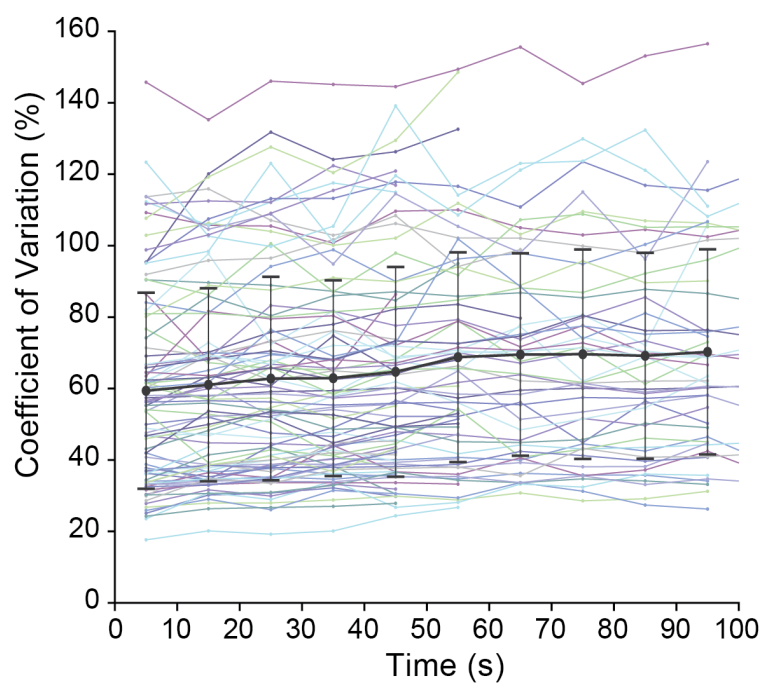

D

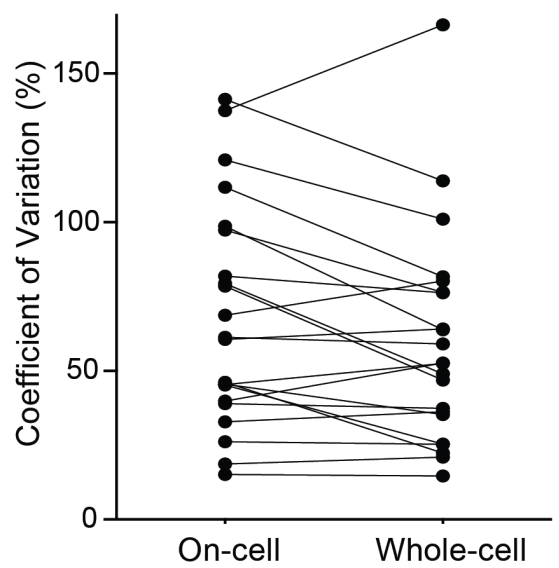

Figure 4.4. Plots of firing activity over time reveal stabilization of activity $\mathbf{5 0}$ seconds after entering the whole-cell configuration. A) First 100 seconds of firing rates were plotted at 10 -second intervals for all identified dopamine neurons. Some neurons had increased firing rate immediately after breakin, but activity stabilized after about 50 seconds. B) CV of all dopamine neurons plotted at 10-second intervals. CV was relatively stable throughout recording but there was a slight, gradual increase in some recorded neurons. Paired comparison of on-cell versus whole-cell recording (at least $50 \mathrm{sec}$. after break-in) firing rates (C) and CV (D) resulted in no significant difference. 
increase in CV (Fig. 4.4B). This indicates that, while the mechanical stress of the membrane breaking and influx of recording solution may lead to transiently increased excitability in DA neurons, it does not disturb their general spike activity. Based on this result, my new dataset consisted of a total of 67 recordings, with the first 50 seconds immediately after break-in excluded from the analysis. The median recording duration for this dataset was 110 seconds (range $=50-140$ ). Paired tests of firing rate and CV for on-cell versus whole-cell recordings from this dataset confirmed that there was no significant difference between the firing rate or $\mathrm{CV}$ of on-cell recordings and that of whole-cell recordings (on-cell FR median(IQR) $=4.33$ (3.73-6.53) Hz, whole-cell FR = $5.28(3.76-6.51) \mathrm{Hz}, \mathrm{p}=0.708$, Wilcoxon signed rank-test; on-cell CV median(IQR) $=60.95$ (38.62-97.69) \%, whole-cell CV $=52.55$ (32.82-77.37) \%, p = 0.0587, Wilcoxon signedrank test; $\mathrm{n}=22, \mathrm{~N}=18$; Fig. $4.4 \mathrm{C}$ and 4.4D). Comparisons of on-cell recordings with extracellular recordings, and on-cell recordings with stable whole-cell recordings suggest that the in vivo mean firing rates and firing patterns of midbrain DA neurons in my dataset are similar to those observed in undisturbed spontaneous activity of extracellularlyrecorded midbrain DA neurons, and that the whole-cell configuration with my internal recording solution does not significantly perturb DA neuron's spontaneous activity. The entire dataset used for subsequent analyses is mapped in Figures 4.5A and 4.5B and its mean firing rates and mean CVs are plotted in Figure 4.5C and 4.5D (FR median $(\mathrm{IQR})=$ $4.80(3.23-6.20) \mathrm{Hz}, \mathrm{CV}$ median(IQR) $=45.99(27.00-64.10) \% ; \mathrm{n}=67, \mathrm{~N}=50)$. The heat maps did not reveal any clear gradient or segregation of FR or CV. Action potential halfwidth was calculated to be 1.52 (1.39-1.69) ms (median(IQR); Fig.4.5E). In addition, firing rates and CV of SNc and VTA DA neurons are plotted in Figure 4.5F and 4.5G, showing higher firing rates and lower CV for VTA DA neurons as compared to those in SNc (SNc FR median $(I Q R)=3.80(2.67-5.15) \mathrm{Hz}$, VTA FR = $5.36(3.98-6.63) \mathrm{Hz}, \mathrm{p}=0.0042$, MannWhitney U test; SNc CV median (IQR) $=60.22$ (39.49-79.29) \%, VTA CV = 37.40 (23.5155.97) \%, $\mathrm{p}=0.0326$, Mann-Whitney U test; SNc $n=28$, VTA $n=39$ ). This is also observed in extracellular recordings of identified DA neurons and further confirms the viability of my whole-cell recordings. 
A

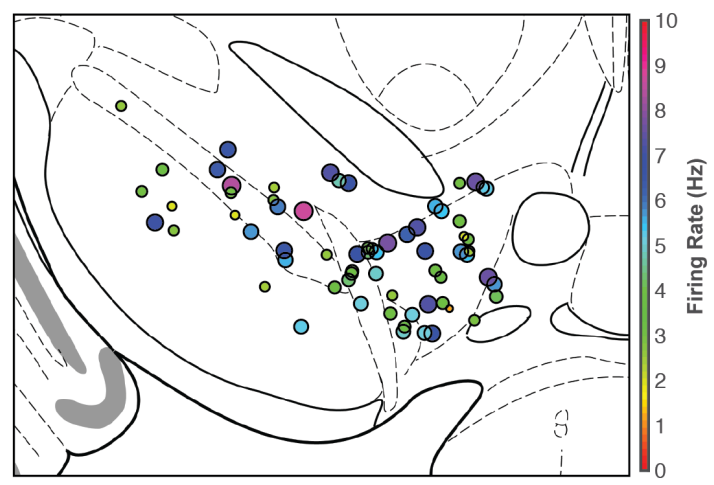

C

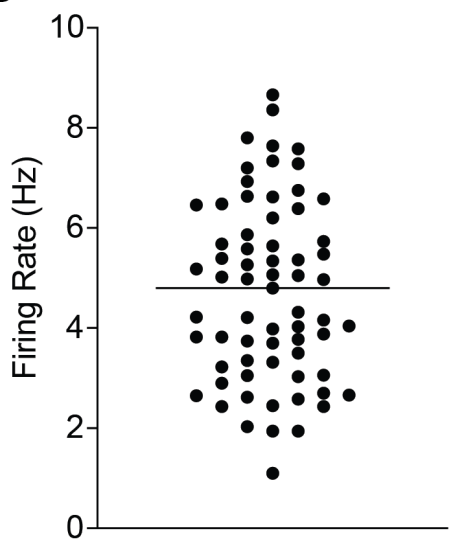

F

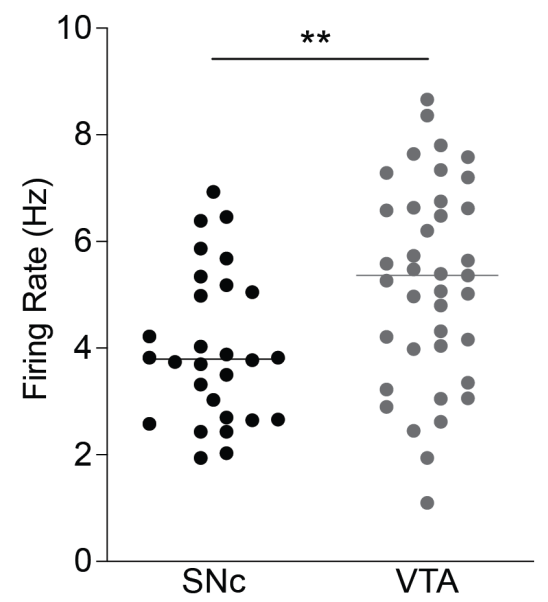

B

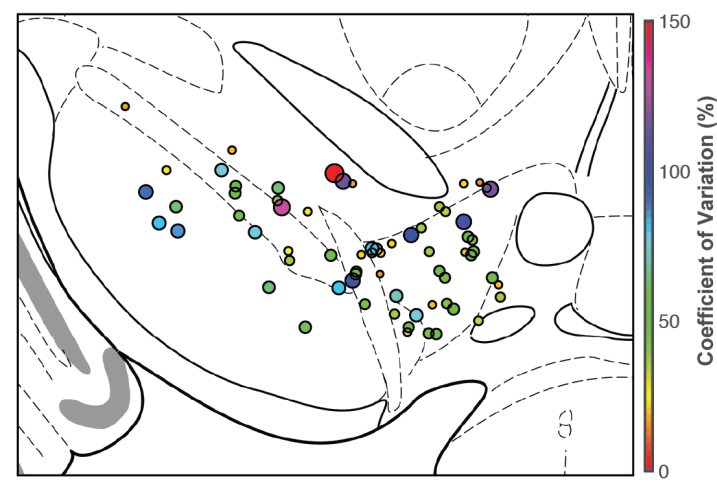

E
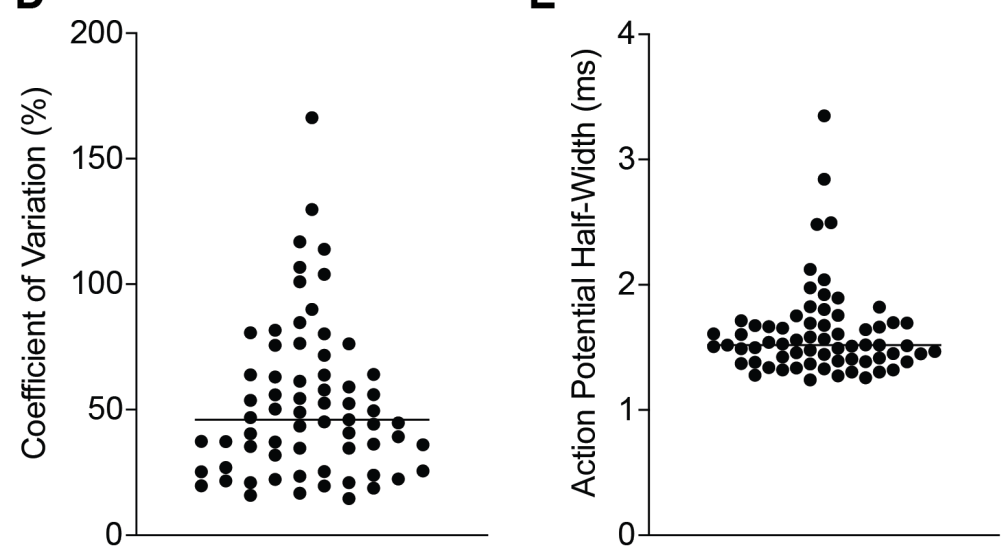

G

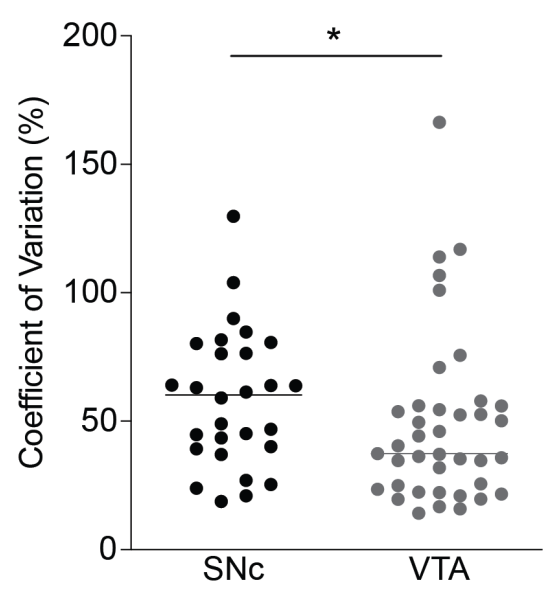

Figure 4.5. General electrophysiological properties of midbrain dopamine neurons. All subsequent quantification and analysis exclude the first 50 seconds of recording immediately following break-in. Anatomical heat maps of mean firing rates $(A)$ and CV (B) from 67 dopamine neurons. Size of circles is scaled to correspond to heat-mapped values for better presentation. Mean firing rates (C), CV (D), action potential half-widths (e.g. widths at half-maximal amplitude) (E). Mean firing rates (F) and CV (G) of dopamine neurons located in the SNc versus VTA showed a slight difference in firing properties. Brain illustrations adapted with permission from G. Paxinos and K. Franklin, 2007. Copyright (2007) Elsevier. 
A
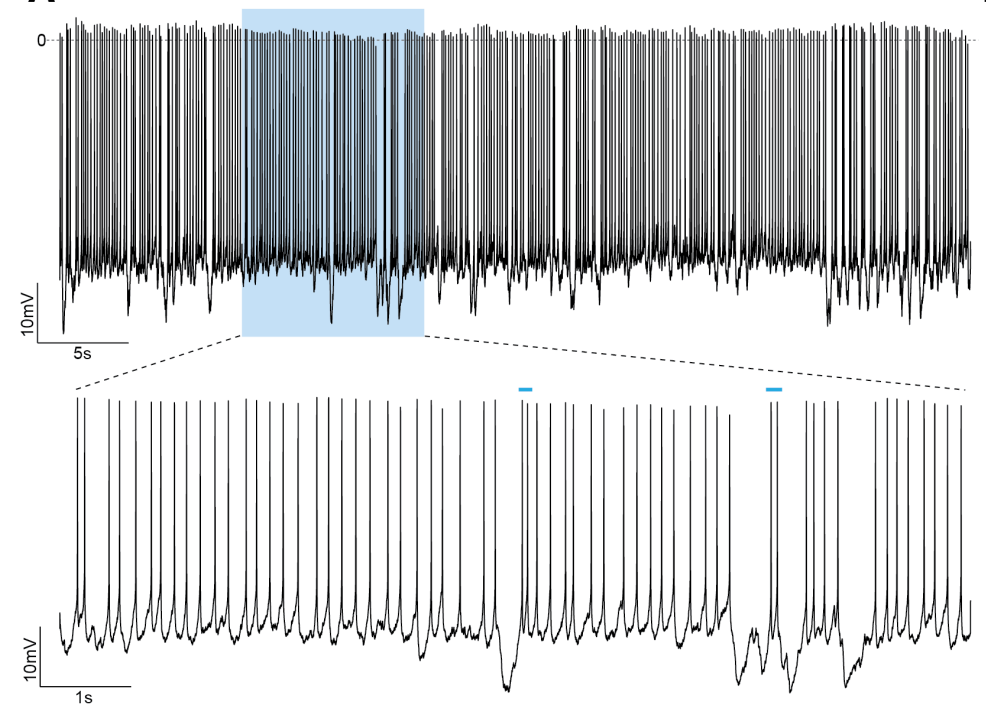

B

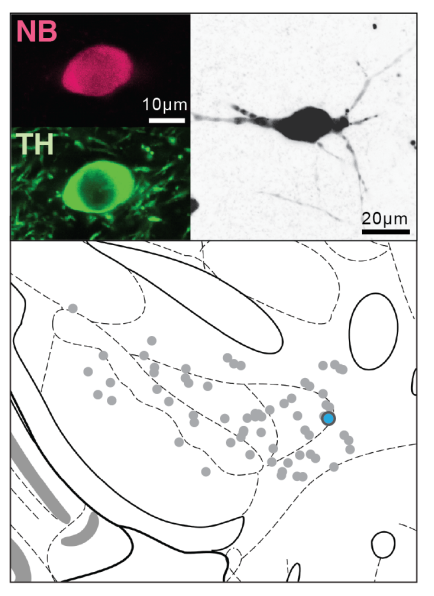

C

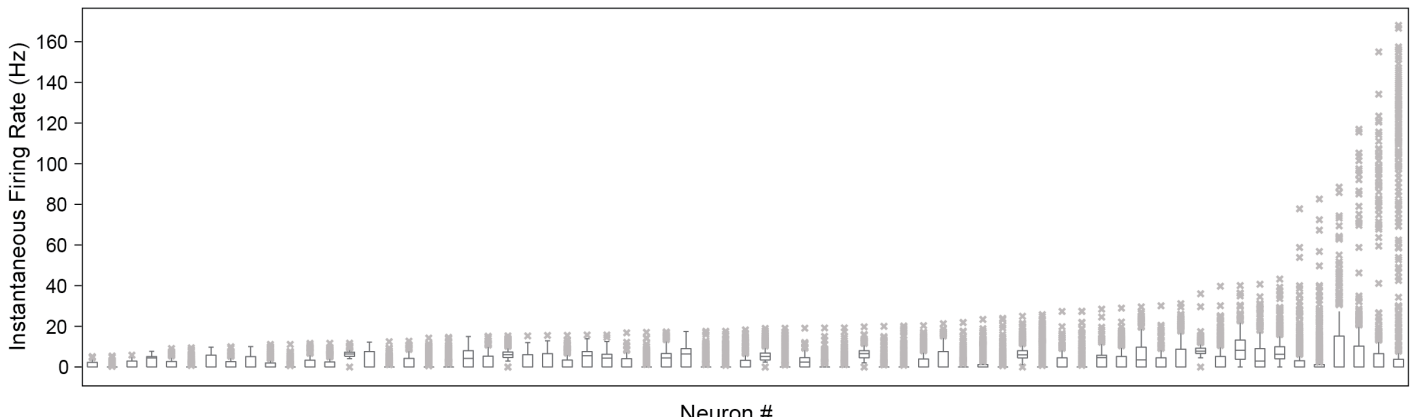

Neuron \#

Figure 4.6. In vivo dopamine neurons exhibit mixed firing patterns. A) Representative recording trace of 50 seconds (top) and 10-second snippet (bottom) show pacemaker activity interspersed with longer ISI and a few bursts (blue lines). B) Top: Confocal images from the dopamine neuron from (A) reveal clear NB and TH co-labeling. Bottom: Anatomical location of the dopamine neuron from (A) shows this recorded neuron was in the VTA. C) Plot showing the full range of instantaneous firing rates of recorded dopamine neurons, which illustrates the wide spectrum of dopamine neuron activity in vivo. NB: neurobiotin; TH: tyrosine hydroxylase. Brain illustration adapted with permission from G. Paxinos and K. Franklin, 2007. Copyright (2007) Elsevier. 


\subsection{Basic subthreshold membrane properties}

Current-clamp recordings of in vivo midbrain DA neurons revealed a wide range of subthreshold membrane activity, most of which was never observed in vitro. Some DA neurons fired in single-spiking patterns but with much more irregularity as compared to in vitro DA neurons, while some neurons showed repetitive rhythmic bursting patterns. Most DA neurons however showed mixed firing patterns consisting of single-spiking and transient bursting. A representative in vivo midbrain DA neuron showing a mixed firing pattern is shown in Figure 4.6A. A 10-second snippet (bottom) from the 50-second trace (top) shows single-spiking activity with occasional burst-like spiking (blue bars) and brief silent periods. This neuron was confirmed to be TH-positive by immunohistochemistry, had dendrites extending in mediolateral directions, and was located in the VTA (Fig. 4.6B). The extensive variability of in vivo DA neuron firing activity can be seen in Figure 4.6C which plots the range of instantaneous firing rates.

DA neurons are known to display the $\mathrm{HCN}$ current $\left(\mathrm{I}_{\mathrm{H}}\right)$ in response to hyperpolarization, but a smaller DA subpopulation has also been shown to lack the sag component which underlies the presence of $\mathrm{I}_{\mathrm{H}}$ (Grace and Bunney, 1984a; Grace and Onn, 1989; Kita et al., 1986; Lammel et al., 2008; Neuhoff et al., 2002). The heterogeneous responses of DA neurons to hyperpolarization have not so far been demonstrated in vivo. To evaluate if in vivo DA neurons would respond to hyperpolarization in a similar manner as in vitro, I carried out step-wise current injection protocols for a subset of recorded neurons. As expected from literature, most DA neurons exhibited a sag component at hyperpolarized membrane potentials $(-80 \mathrm{mV})$, while their rebound responses were more diverse in comparison to those observed in vitro and ranged from no notable response to a long delay to bursting. Three example DA neurons exhibiting a different response to hyperpolarizing current injection are illustrated in Figure 4.7: a lateral SNc DA neuron with a large sag component but no rebound delay (Fig. 4.7A), a VTA DA neuron with small sag and some rebound delay (Fig. 4.7B), and a medial VTA DA neuron with no sag and a long rebound delay (Fig. 4.7C). Unlike previously shown for projection-defined in vitro DA neurons (Lammel et al., 2008), in which they had two main phenotypes ('sag and short rebound delay' and 'no sag and long rebound delay'), in vivo DA neurons' response to hyperpolarization were not clearly segregated. Sag amplitudes and rebound 
delays of a subset of in vivo DA neurons are plotted in Figure 4.7D and 4.7B, respectively $($ Sag median $(\mathrm{IQR})=9.00$ (6.98-10.82) mV; Rebound delay median(IQR) = 91.78 (61.65267.1) ms; $n=46, N=40$ ). Sag amplitude was higher in DA neurons located in the medial SNc and lateral portion of VTA (Fig. 4.7G). Rebound delay was longer in medial VTA DA neurons (Fig. 4.7H), but there were also two neurons in the lateral SNc that showed longer rebound delay resulting from failed spikes, unlike the straight rebound slopes observed in medial VTA DA neurons. The wide spectrum of in vivo DA neurons' response to hyperpolarization and rebound behavior is likely the result of a combination of their intrinsic properties and synaptic influence. Furthermore, input resistance for this subset of DA neurons was calculated to be approximately $280 \mathrm{M} \Omega$ (Input resistance median $(\mathrm{IQR})=277.5$ (233.9-322.6) $\mathrm{M} \Omega ; \mathrm{n}=46, \mathrm{~N}=40$ ) (Fig. 4.7F), which was in stark contrast to the previously reported value of $31 \mathrm{M} \Omega$ for intracellularly-recorded in vivo DA neurons (Grace and Bunney, 1983b), and was closer to the input resistance of synaptically-isolated in vitro DA neurons (approximately $450 \mathrm{M} \Omega$ using the same recording solution). The relatively high input resistance would suggest that DA neurons are more resistant to shunting than previously thought and in comparison to other types of neurons (Tripathy et al., 2015).

Figure 4.7. Dopamine neurons display a range of subthreshold membrane activity upon hyperpolarization. Traces shown are from injection protocols in which negative current of increasing amplitude was injected into the cell in a step-wise fashion. Dotted lines indicate $-80 \mathrm{mV}$. Injected current is noted next to trace. Beginning of rebound delay is marked with an arrow. Each example neuron is assigned a color (blue, green and orange) which is used to demonstrate their corresponding values in plots D-F. A) Top: Example trace showing a large sag component and a short rebound delay in response to hyperpolarization. Bottom: Histology and location of this neuron. B) Top: Trace showing a combination of small sag and some rebound delay. Bottom: Histology and location of this neuron. C) Top: Trace showing no sag and a long rebound delay during hyperpolarization. Bottom: Histology and location. Plots of sag amplitude (D), rebound delay (E) and input resistance (F) from all dopamine neurons in which hyperpolarization protocols were run. Anatomical heat maps of sag amplitude (G) and rebound delay $(\mathrm{H})$. Longer rebound delays are localized to the medial VTA and lateral SNc. Brain illustrations adapted with permission from G. Paxinos and K. Franklin, 2007. Copyright (2007) Elsevier. 

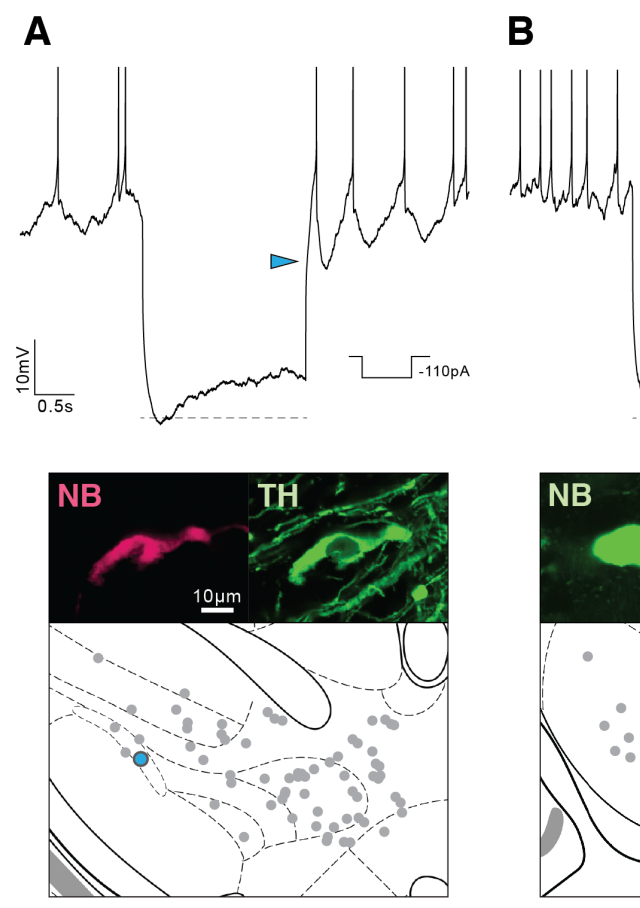

D

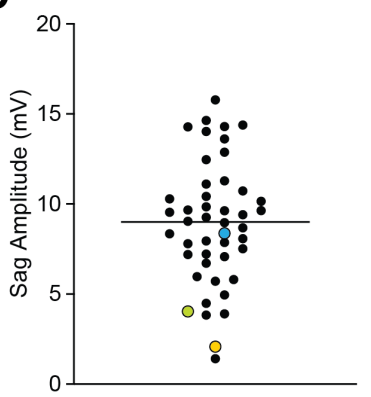

G

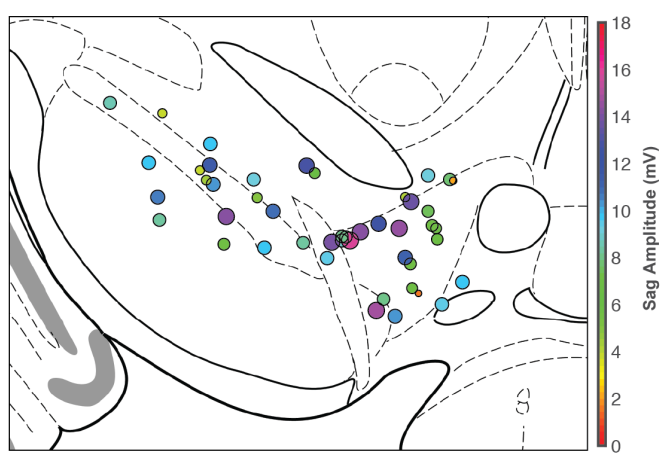

E
C
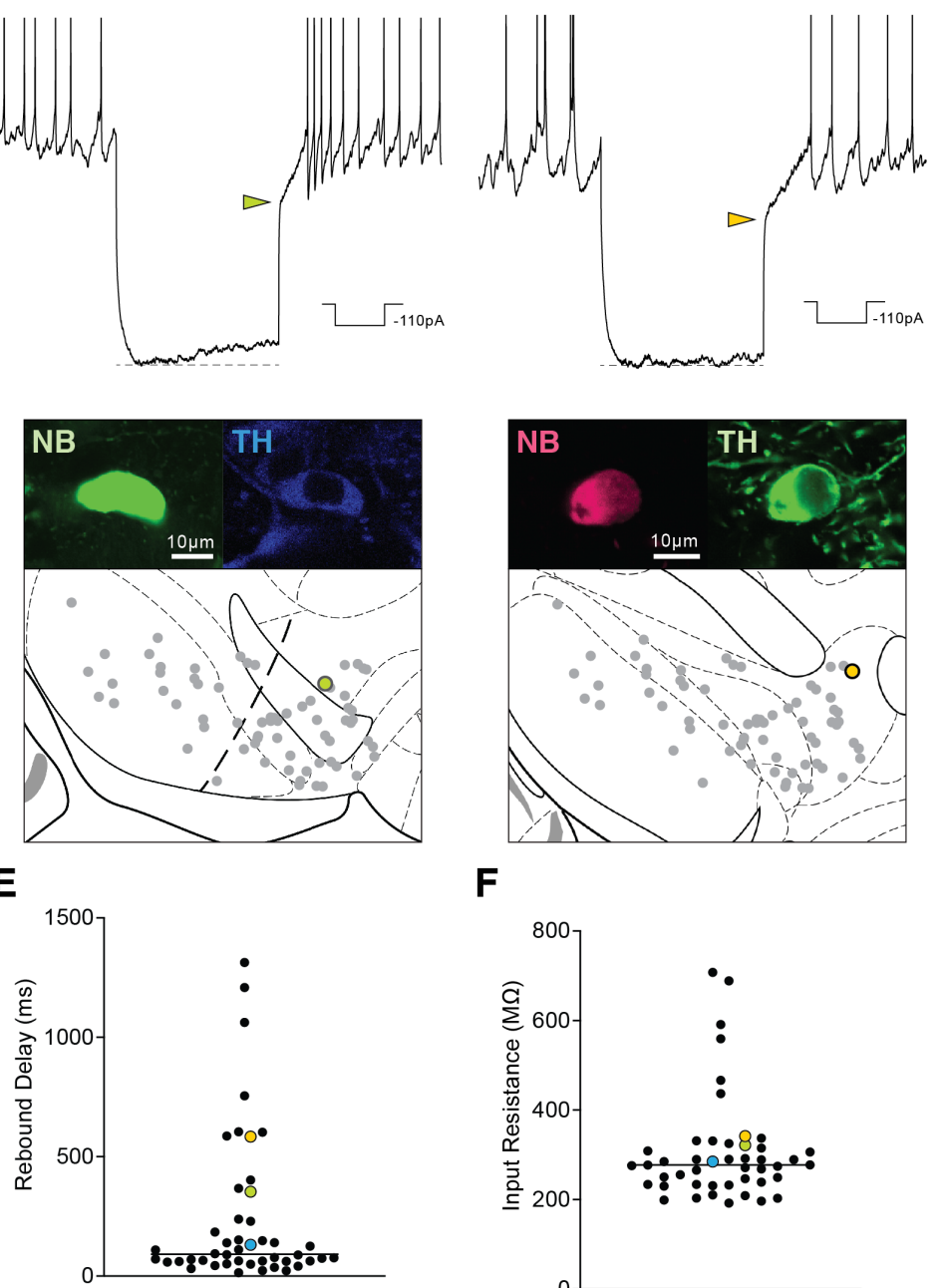

H

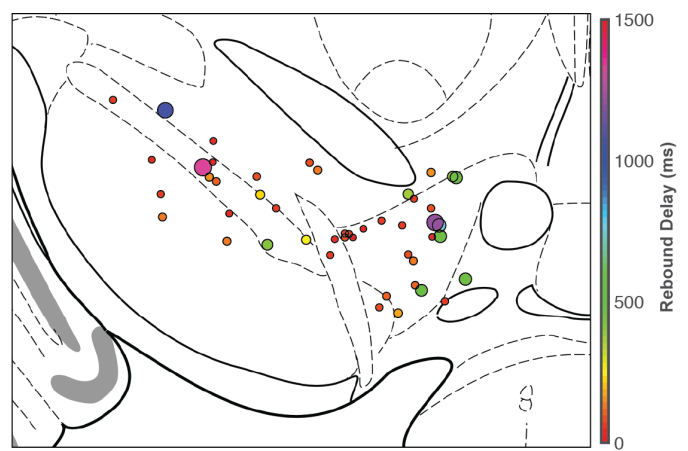




\subsection{Subthreshold membrane signatures of tonic and phasic firing}

My initial observation of raw traces led to the hypothesis that spontaneous firing activity of in vivo midbrain DA neurons was closely related to fluctuations in the subthreshold membrane potentials. Moreover, hyperpolarized membrane potentials, which were generally the basis of longer ISI durations, were often followed by higher frequency burst-like firing, which can be seen in the Figure 4.6A example. Conventionally, "burst firing" of DA neurons is identified based on the ISI criteria proposed by Grace and Bunney (Grace and Bunney, 1984b). However, subthreshold membrane dynamics could potentially provide sufficient information to identify distinct tonic and phasic firing patterns of in vivo DA neurons in a subthreshold activity-dependent fashion. Thus, to first characterize the subthreshold membrane activity of in vivo DA neurons during spontaneous activity, I plotted the histograms of ISI membrane potential minima $\left(\mathrm{V}_{\min }\right)$, and additionally histograms of action potential thresholds $\left(\mathrm{V}_{\text {thr }}\right)$ to see if $\mathrm{V}_{\text {thr }}$ also fluctuated along with $V_{\text {min }}$. As expected, the $V_{\min }$ and $V_{\text {thr }}$ histograms of some DA neurons exhibited clear unimodal or multimodal distributions, but interestingly, $V_{\min }$ and $V_{\text {thr }}$ did not always exhibit identical distribution patterns. In fact, a multimodal distribution of $V_{\text {thr }}$ appeared to underlie another type of burst firing from that of bimodal $\mathrm{V}_{\min }$. Based on these findings, I identified three distinct types of in vivo subthreshold membrane signatures in DA neurons that are the basis of their firing patterns: 1) unimodal $V_{\min }$ and $\mathrm{V}_{\text {thr }}$ distributions, 2) a bimodal $\mathrm{V}_{\min }$ distribution and a unimodal $\mathrm{V}_{\text {thr }}$ distribution, and 3) a bimodal/multimodal $\mathrm{V}_{\min }$ distribution and a bimodal $\mathrm{V}_{\text {thr }}$ distribution. Recording traces of DA neurons that clearly showed one of the three signatures were further analyzed to examine their properties in detail. Each class will be discussed in depth using a representative DA neuron belonging to the class.

An example trace from the first class of DA neurons is shown in Figure 4.8A. This neuron showing a highly regular pacemaker firing pattern was fusiform in shape and located in the VTA (Fig. 4.8B). Blue circles and green circles in Figure 4.8A indicate $V_{\text {min }}$ and $V_{\text {thr, }}$, respectively, which illuminate their regularity with almost no fluctuations. The narrow unimodal distribution of the $V_{\min }$ histogram (Fig. 4.8C) and the even narrower distribution of the $\mathrm{V}_{\text {thr }}$ histogram (Fig. 4.8D) demonstrate that the highly stable, regular $V_{\min }$ and $V_{\text {thr }}$ underlie this neuron's pacemaker firing pattern. The two distributions were 
fitted to a Gaussian curve for variance quantification; the standard deviation (SD, $\sigma$ ) for the fit was $1.14 \mathrm{mV}$ for $V_{\min }(\mu=-43.36 \mathrm{mV})$ and $0.61 \mathrm{mV}$ for $V_{\text {thr }}(\mu=-29.78 \mathrm{mV})$. This class of DA neurons with narrow unimodal $V_{\min }$ and $V_{\text {thr }}$ distributions is considered tonic firing.

A

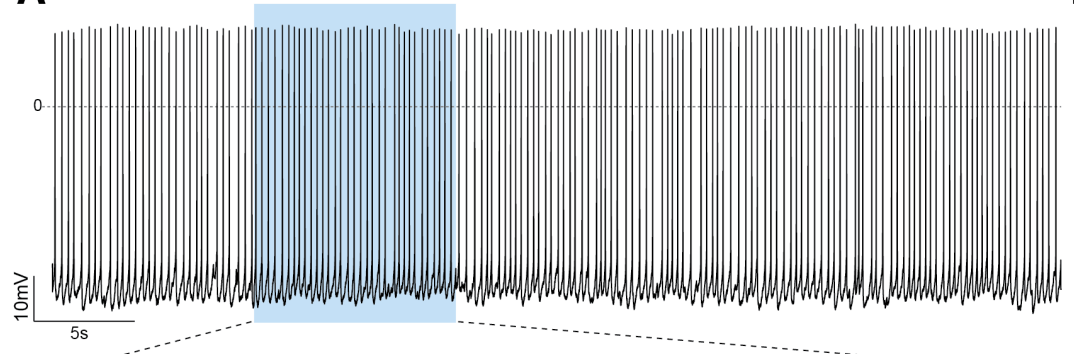

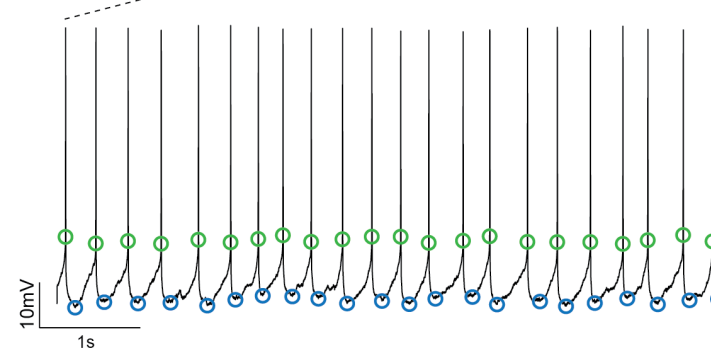

C

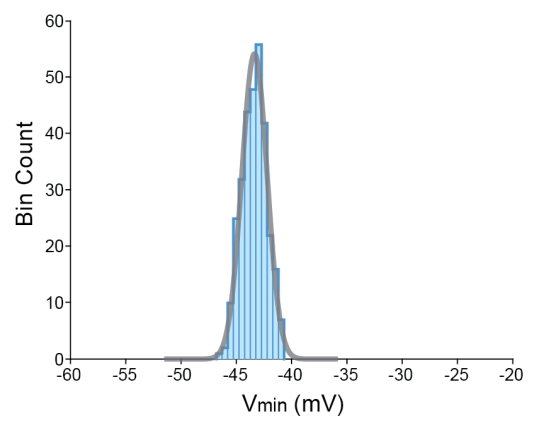

B

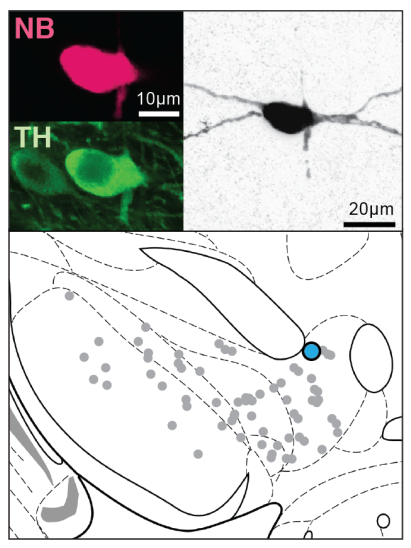

Figure 4.8. Dopamine neurons with unimodal $\mathrm{V}_{\min }$ and $\mathrm{V}_{\mathrm{thr}}$ distributions display pacemaker firing. Dopamine neurons were categorized into 3 groups based on their subthreshold membrane signatures $\mathrm{V}_{\min }$ and $\mathrm{V}_{\mathrm{thr}}$. A) Top: Example 50-second trace of a representative dopamine neuron with unimodal $V_{\min }$ and $V_{\text {thr }}$ distributions. Bottom: 10-second snippet of the top trace. Blue circles represent $V_{\min }$ and green circles $V_{\text {thr }}$, both of which are highly stable. B) Top: Histology of the neuron in (A). Bottom: Location of this neuron was in the VTA. C) Gaussian curve (gray) was fitted to the histogram of $V_{\text {min }}$, which shows a clear unimodal distribution. D) Histogram of $\mathrm{V}_{\text {thr }}$ shows an even narrower unimodal distribution. This group of dopamine neurons is considered tonic/papermaker firing. Brain illustration adapted with permission from G. Paxinos and K. Franklin, 2007. Copyright (2007) Elsevier. 
A representative recording of an in vivo DA neuron from the second class, characterized by a bimodal $V_{\min }$ distribution and a unimodal $V_{\text {thr }}$ distribution, is shown in Figure 4.9A. The bottom trace illustrates a rhythmic firing pattern featuring non-spiking periods with pronounced hyperpolarizations leading to depolarization waves where several burst-like spikes occur. As reported in literature discussing burst firing (Grace and Bunney, 1984a, 1984b), these spikes showed decreasing amplitudes. This neuron also had a fusiform shape and was located in the lateral SNc (Fig. 4.9B). $V_{\text {min }}$ (blue circles) fluctuates at two membrane voltage levels almost $10 \mathrm{mV}$ apart, while $\mathrm{V}_{\text {thr }}$ (green circles) follows a relatively straight trajectory but with more variabilities as compared to that of pacemaking. The $V_{\min }$ and $V_{\text {thr }}$ distributions from this neuron are shown in Figures $4.9 \mathrm{C}$ and 4.9D, respectively. The $\mathrm{V}_{\min }$ of more hyperpolarized membrane potentials form the Mode 1 distribution (Fig. 4.9C, pink curve), while the $V_{\min }$ of less hyperpolarized membrane potentials form the Mode 2 (Fig. 4.9C, gray curve), which is probably close to the neuron's resting membrane potentials, based on its larger distribution and the voltage range. The SD of the two Gaussian fits was $1.49 \mathrm{mV}$ for Mode $1(\mu=-44.71 \mathrm{mV})$ and 1.08mV for Mode $2(\mu=-34.02 \mathrm{mV})$. The bimodality of $V_{\min }$ resulted in a $V_{\text {min }}$ range that was much broader in comparison to the earlier pacemaker example. In contrast, $\mathrm{V}_{\text {thr }}$ has a narrow unimodal distribution which was mostly unaffected by the fluctuating $\mathrm{V}_{\text {min }}$ $(\mu \pm \sigma=-25.27 \pm 1.56 \mathrm{mV})$. Due to the nature of their bursts, these DA neurons with a bimodal $\mathrm{V}_{\min }$ and unimodal $\mathrm{V}_{\text {thr }}$ are considered rebound bursters. 
A
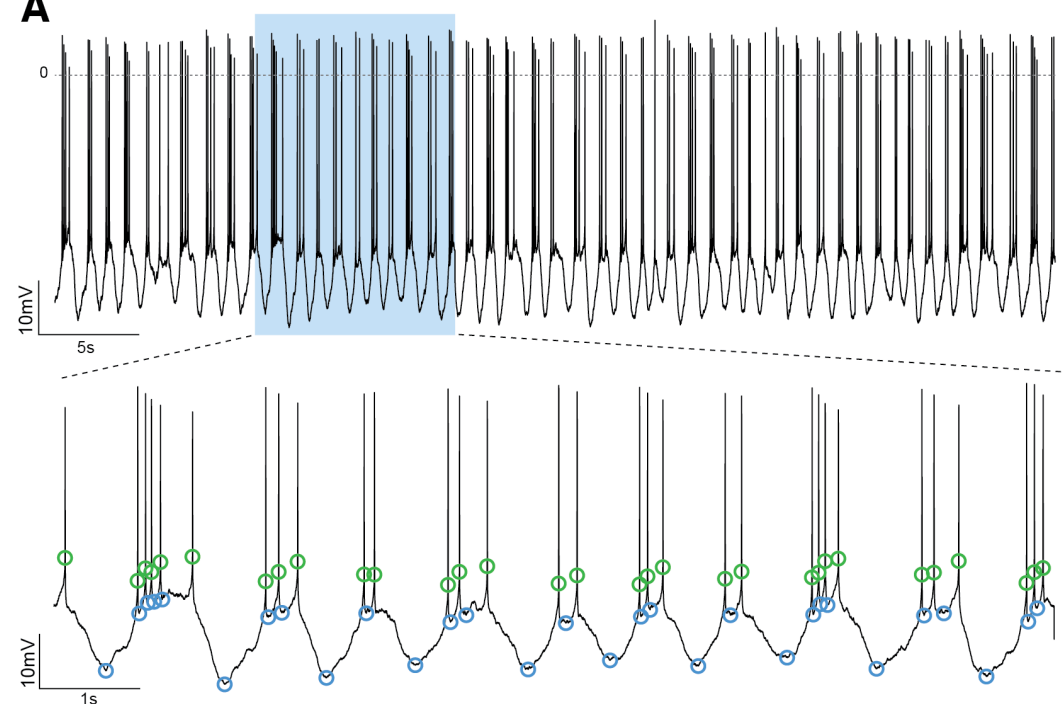

C

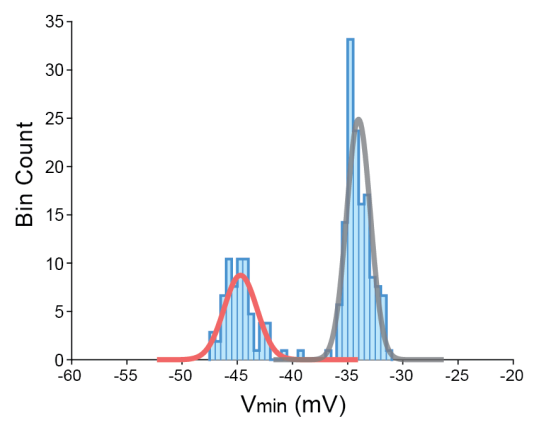

B

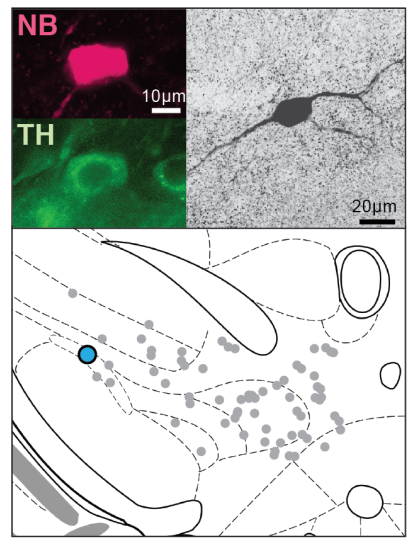

D

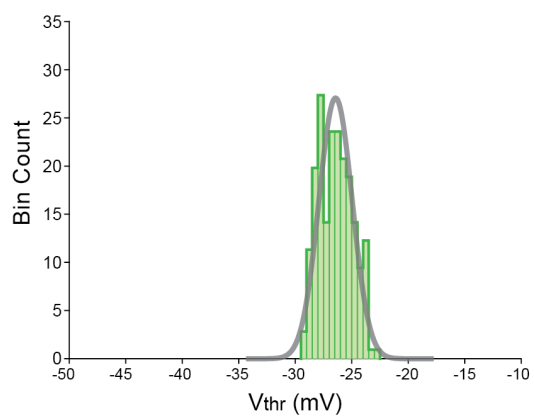

Figure 4.9. Dopamine neurons with bimodal $\mathbf{V}_{\min }$ and unimodal $\mathbf{V}_{\text {thr }}$ distributions show rebound burst firing. A) Top: Example 50-second trace of a dopamine neuron with bimodal $V_{\min }$ and unimodal $\mathrm{V}_{\text {thr }}$ distributions. Bottom: 10 -second snippet of the top trace. $\mathrm{V}_{\min }$ is in blue circles and $\mathrm{V}_{\text {thr }}$ in green circles. $V_{\min }$ fluctuates at two membrane potential levels, while $V_{t h r}$ does not. B) Top: Histology of the neuron in (A). Bottom: This neuron was found in the lateral SNc. C) Histogram of $\mathrm{V}_{\min }$ display a clear bimodal distribution. Pink Gaussian curve indicates Mode 1. D) Histogram of $V_{\text {thr }}$ shows a unimodal distribution. This group of dopamine neurons is considered rebound bursters. Brain illustration adapted with permission from G. Paxinos and K. Franklin, 2007. Copyright (2007) Elsevier. 
The third class of DA neurons is characterized by a bimodal/multimodal $\mathrm{V}_{\text {min }}$ distribution and a bimodal $\mathrm{V}_{\text {thr }}$ distribution. Figure 4.10A shows an example recording of this type of neuron. The 10-second trace illustrates a repetitive firing pattern, with highfrequency spikes occurring at transient plateau membrane potentials (Fig. 4.10A, bottom). This neuron was more round in shape and located in the dorsal tier VTA (Fig. 4.10B). Here, $\mathrm{V}_{\text {thr, }}$, represented by green circles in Figure 4.10A, fluctuate considerably throughout the recording, going back-and-forth between low and high membrane potential ranges. $\mathrm{V}_{\min }$, marked as blue circles, also have large shifts and at times become more depolarized than $\mathrm{V}_{\text {thr. }}$. The membrane potentials appeared to be noisier in contrast to pacemaker firing or rebound bursting examples. The histogram of $\mathrm{V}_{\text {min }}$ revealed a complex distribution, which was approximated to have a bimodal or possibly trimodal shape, with a much broader $\mathrm{V}_{\text {min }}$ range as compared to pacemaker or rebound bursting neurons (Fig. 4.10C). The $\mathrm{V}_{\text {thr }}$ is bimodally distributed with the Mode 1 distribution having a narrower range than that of Mode 2 (Fig. 4.10D, gray curve and pink curve, respectively). The SD for the two $\mathrm{V}_{\text {thr }}$ Gaussian fits were $0.90 \mathrm{mV}$ for Mode 1 and $1.85 \mathrm{mV}$ for Mode 2 ( $\mu=-33.30$ and $-26.56 \mathrm{mV}$, respectively). I termed this class of DA neurons characterized by a bimodal $\mathrm{V}_{\text {thr }}$ distribution plateau bursters. 
A
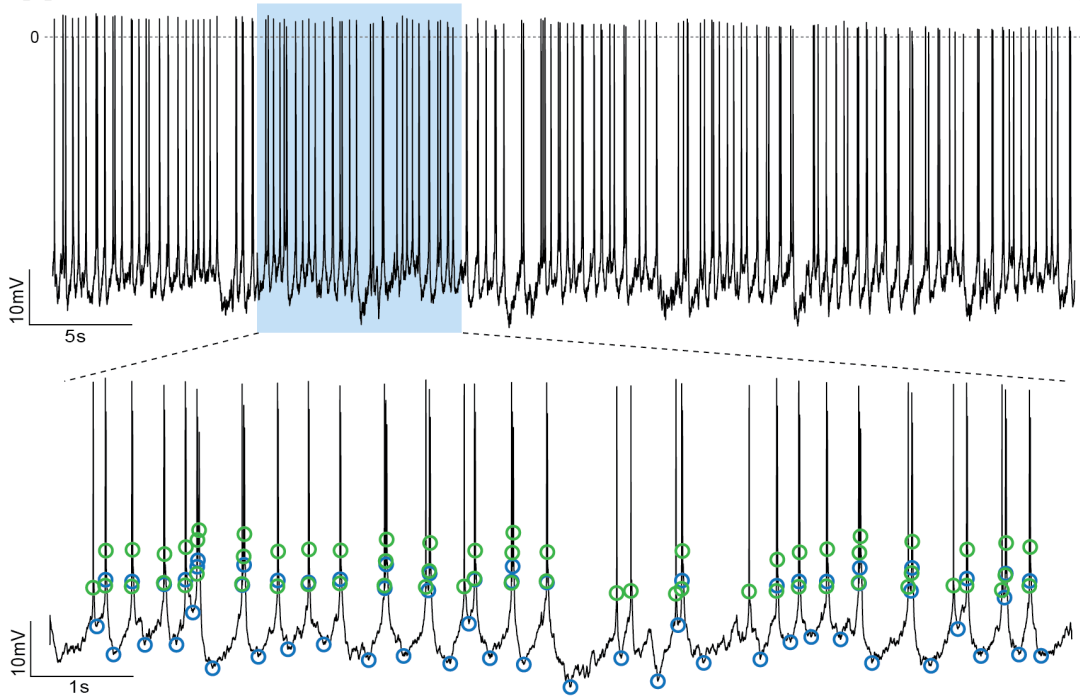

C

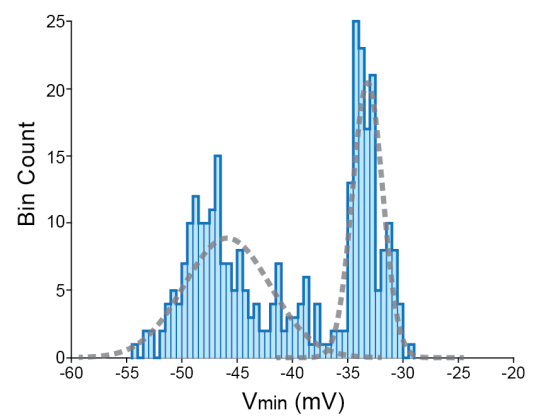

B

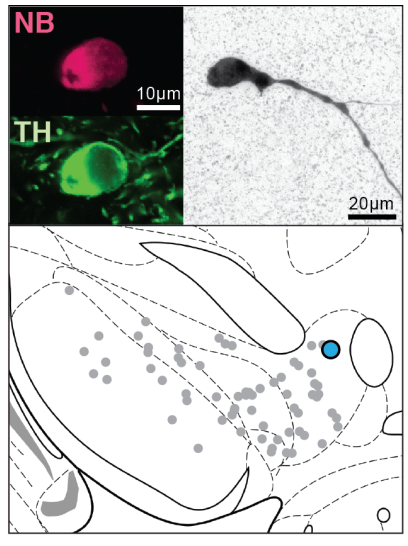

Figure 4.10. Dopamine neurons with multimodal $\mathrm{V}_{\min }$ and bimodal $\mathrm{V}_{\text {thr }}$ distributions display plateau bursting. A) Top: Example 50-second trace of a dopamine neuron with bimodal $V_{\min }$ and $V_{\text {thr }}$ distributions. Bottom: 10 -second snippet of the top trace. Blue circles represent $V_{\min }$ and green circles $\mathrm{V}_{\text {thr. Note }}$. that both $\mathrm{V}_{\min }$ and $\mathrm{V}_{\mathrm{thr}}$ are fluctuating at different membrane potential levels. B) Top: Histology of the neuron in (A). Bottom: This neuron was located in the VTA. C) Histogram of $V_{\text {min }}$ shows a bimodal/multimodal distribution with large variabilities, which was fitted with two Gaussian curves (dotted lines). D) Histogram of $\mathrm{V}_{\mathrm{thr}}$ exhibits a clear bimodal distribution. Pink curve indicates Mode 2. This group of dopamine neurons is considered plateau bursting. Brain illustration adapted with permission from G. Paxinos and K. Franklin, 2007. Copyright (2007) Elsevier. 
Based on the observation that hyperpolarized membrane potentials in rebound bursts led to longer ISIs and depolarized plateau bursts led to shorter ISIs, I plotted ISIs as $\log _{10}$ against their corresponding $V_{\min }$ and $V_{\text {thr }}$ (with $V_{\text {thr }}$ coupled to preceding ISI) to evaluate their relationship and further characterize the subthreshold membrane dynamics of the three types of DA neurons. Unsurprisingly, for the pacemaker neuron examined in Figure 4.8, scatterplots of ISI versus $V_{\min }$ and $V_{\text {thr }}$ each formed a small, dense cluster (Fig. 4.11A). To determine if there is a regularity or pattern that can be found within these ISI- $V_{\min }$ and ISI- $V_{\text {thr }}$ clusters, the same data were plotted as a continuous line in the right sequence of events. As evident from the random trajectory of the plot, there was no systematic trend or pattern to the data (Fig. $4.11 \mathrm{~B}$, only ISI- $V_{\min }$ is shown). To visually demonstrate the subthreshold membrane behavior underlying the regularity of this neuron, event-triggered averaging was carried out, in which multiple spikes were aligned at the time of action potential threshold (Fig. 4.11C). The averaged trace, shown in Fig. 4.11D, revealed the highly stereotypical subthreshold membrane activity surrounding action potentials from pacemaker neurons. Resembling in vitro activity, action potentials of this in vivo DA neuron are preceded by a slow depolarization and followed by a prominent afterhyperpolarization (AHP) that leads to the next action potential cycle. To characterize the action potential kinetics of this neuron, three-spike sequences, consisting of non-overlapping three action potential sequences from this neuron were further analyzed. Figure 4.11E shows an example three-spike sequence, with Spike 0 (blue) providing the basis of comparison, and preceding and succeeding spikes of Spike 0 designated as Spike -1 (light blue) and Spike +1 (purple), respectively. Figure $4.11 \mathrm{~F}$ illustrates spike waveforms of 50 three-spike sequences aligned at the time of action potential thresholds, with averaged traces shown in bold. The averaged traces of Spike -1 , Spike 0 , and Spike +1 were aligned at the spike threshold voltage in Figure 4.11G. Throughout firing, action potential waveforms maintained their shape in this pacemaker neuron. As expected, the phase plot of the rate of change in membrane potentials over time $(\mathrm{dV} / \mathrm{dt})$ against the membrane potential voltage $(\mathrm{Vm})$ for threespike sequences also indicated that this neuron's action potential threshold kinetics are steady throughout three-spike sequences (Fig. 4.11H). Spike thresholds and dV/dt maxima of three-spike sequences also remained stable $\left(\mathrm{V}_{\text {thr: }}\right.$ Spike -1 median(IQR) = - 
20.83(-30.23 to -29.42$) \mathrm{mV}$, Spike $0=-29.90(-30.26$ to -29.62$) \mathrm{mV}$, Spike $+1=-30.01(-$ 30.28 to -29.49 ) $\mathrm{mV}$; Spike 0 vs. Spike $-1 \mathrm{p}=0.396$, Spike 0 vs. Spike $+1 \mathrm{p}=0.709$, Wilcoxon signed-rank test; dV/dt maxima: Spike -1 median(IQR) = 55.43(54.21-56.44) $\mathrm{V} / \mathrm{s}$, Spike $0=55.54(54.19-56.89) \mathrm{V} / \mathrm{s}$, Spike $+1=55.78(53.99-56.99) \mathrm{V} / \mathrm{s}$; Spike 0 vs. Spike $-1 p=0.871$, Spike 0 vs. Spike $+1 p=0.484$, Wilcoxon signed-rank test; $n=50$ ) (Fig. 4.11I and 4.11J). The results show that DA neurons have a great capacity to maintain stable, autonomous spiking as in vitro, despite the presence of synaptic inputs as well as neuromodulatory influence.

Figure 4.11. Pacemaker pattern of in vivo dopamine neurons exhibits great stability in subthreshold membrane behavior. A) Scatterplots of $\log _{10} I S I$ against $V_{\text {min }}$ (blue) and against $V_{\text {thr }}$ (green). B) Random snippet of ISI- $V_{\min }$ in (A) was plotted as a continuous line, which shows no clear pattern or trend. C) 10 random non-overlapping traces were superimposed at the arrow to reveal stereotypical subthreshold membrane behavior surrounding spikes. D) Mean and SD of (C) reveals subthreshold membrane dynamics surrounding pacemaking spikes. Blue circle indicates $V_{\text {thr. }}$ E) Example three-spike sequence further analyzed. F) All spikes from 50 three-spike sequences (= 150 spikes total) were superimposed and aligned at the time of $\mathrm{V}_{\mathrm{thr} .}(+)$ symbol indicates $\mathrm{V}_{\text {thr. }}(\mathrm{x})$ symbol indicates maximal rate of depolarization (max. $\mathrm{dV} / \mathrm{dt}$ ). G) Averaged traces of (F) normalized to the $\mathrm{V}_{\mathrm{thr}}$ voltage, which shows that Spike -1 , Spike 0 and Spike +1 are essentially identical. H) Phase plot showing the change in rate of depolarization against membrane potentials. Plots of $\mathrm{V}_{\text {thr }}$ during three-spike sequences (I) and change in max. $\mathrm{dV} / \mathrm{dt}(\mathrm{J})$ show no notable patterns during spike sequences in dopamine neurons firing in the pacemaker mode. 
A

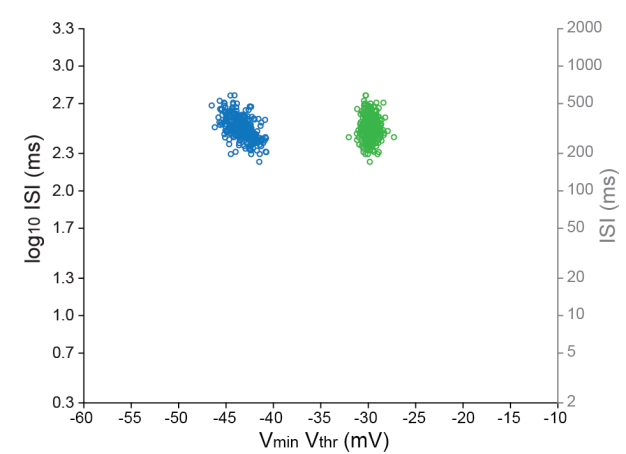

C

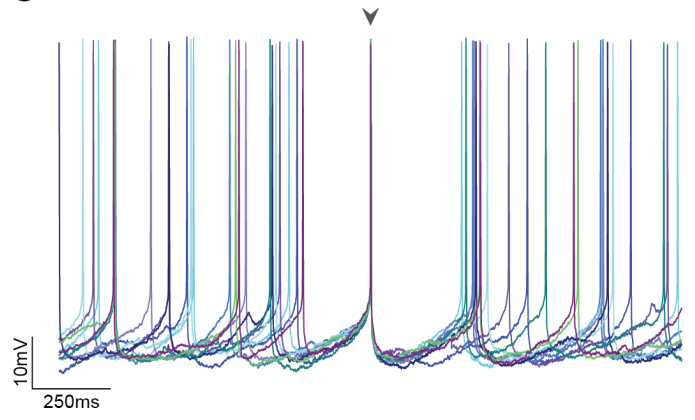

B

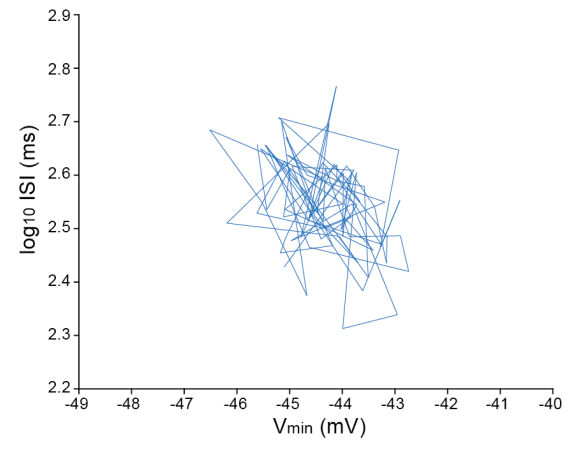

D

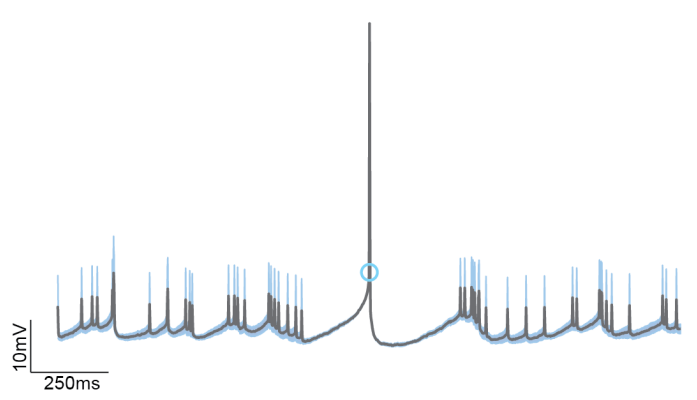

E

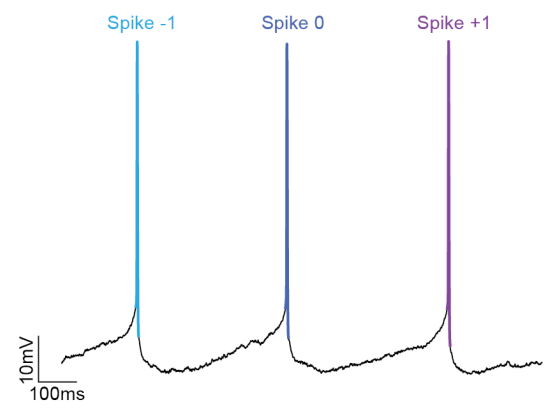

H

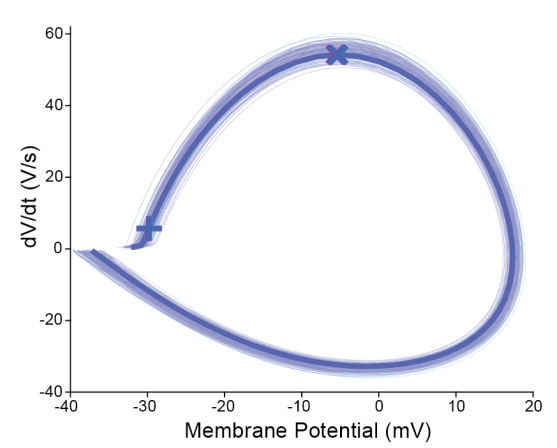

F
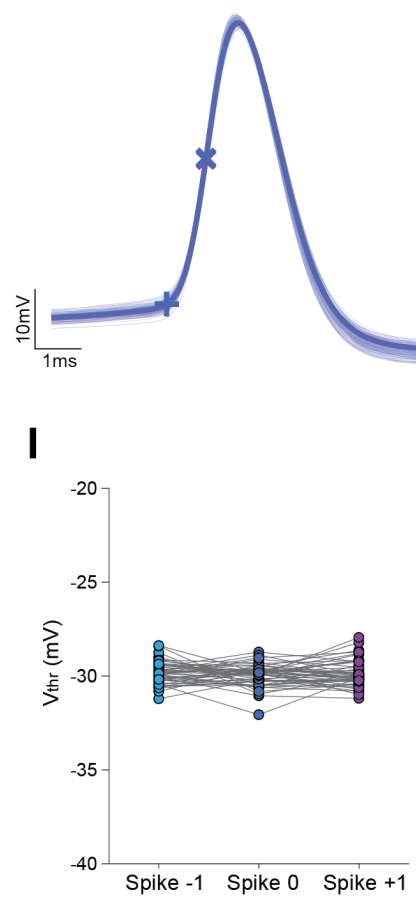

G

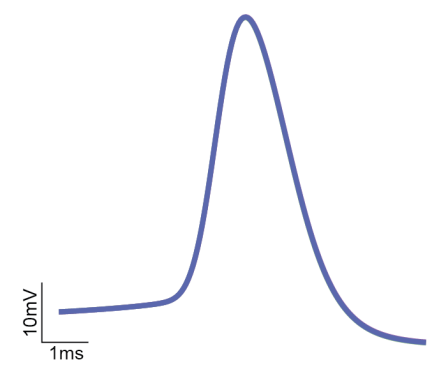

J

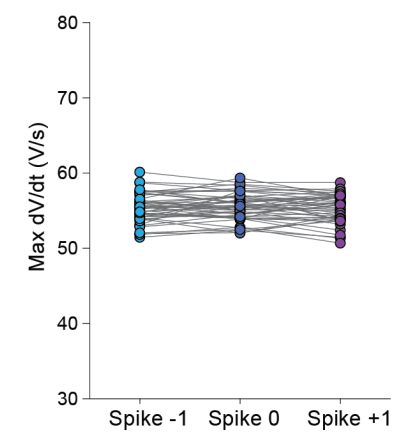


In regard to rebound bursting, the scatterplot of ISI versus $V_{\min }$ displayed a nonlinear correlation and formed two clearly-segregated clusters (Fig. 4.12A, blue), with longer ISIs coupled to hyperpolarized membrane potentials creating a smaller Cluster 1 (pink centroid) and shorter ISIs coupled to more depolarized membrane potentials forming a larger Cluster 2 (black centroid), as identified by hierarchical clustering. For comparison, ISI versus $\mathrm{V}_{\text {thr }}$ was also plotted which revealed a highly-dense cluster in the longer ISI range corresponding to the ISI- $\mathrm{V}_{\min }$ Cluster 1 (Fig. 4.12A, green). However, due to the narrow range of $V_{\text {thr }}$, the plot did not segregate the two clusters in the $V_{\text {thr }}$ axis. To see if the two ISI- $V_{\min }$ clusters show a pattern, a line plot was created in the right sequence of events, which revealed that ISI- $V_{\min }$ Cluster 1 spikes and ISI-V $V_{\min }$ Cluster 2 spikes residing in the short ISI/depolarized $\mathrm{V}_{\min }$ range almost always fired in sequence (Fig. 4.12B). Next, to uncover the stereotypical subthreshold signature of rebound bursting, event-triggered averaging was implemented for spikes residing in ISI- $V_{\min }$ Cluster 1 . Ten random spikes from ISI- $V_{\min }$ Cluster 1 are superimposed in Figure 4.12C. The averaged trace in Figure 4.12D illustrates a prominent hyperpolarization preceding the aligned spike with a remarkable low-variability in subthreshold membrane activity, particularly from the point of $V_{\min }$ (light blue arrow) to the slow depolarization leading to the subsequent spike. To reveal the dynamic change of action potential kinetics in rebound bursting, I further analyzed rebound burst spikes and their surrounding spikes as a sequence. Spike 0 represents the spike from ISI-V $V_{\min }$ Cluster 1 (Fig. 4.12E, blue), its preceding spike is designated as Spike -1 (light blue), and the succeeding spike is designated as Spike +1 (purple). All action potentials in 50 three-spike sequences are aligned at the time of spike threshold in Figure 4.12F, which show that each spike in the sequence has a different action potential waveform as well as action potential threshold, with Spike -1 having the most depolarized threshold and Spike 0 having the most hyperpolarized threshold. Rebound burst sequences also showed a dynamic change in the amplitude and AHP, and Spike 0 displayed the largest amplitude and the fastest repolarization (Fig. 4.12G). The phase plot of the same sequences shows that, in contrast to tonic firing, the spike kinetics for rebound bursting changed dynamically during threespike sequences (Fig. 4.12H). $\mathrm{V}_{\text {thr }}$ of Spike 0 were more hyperpolarized as compared to those of Spike -1 , but in the following Spike +1 they already shifted to more depolarized 
potentials $\left(\mathrm{V}_{\mathrm{thr}}\right.$ : Spike -1 median(IQR) $=-25.07(-25.87$ to -24.09$) \mathrm{mV}$, Spike $0=-28.03(-$ 28.28 to -27.68$) \mathrm{mV}$, Spike $+1=-26.54(-26.88$ to -26.27$) \mathrm{mV}$; Spike 0 vs. Spike $-1 \mathrm{p}$ $<0.0001$, Spike 0 vs. Spike $+1 \mathrm{p}<0.0001$, Wilcoxon rank-sum test; $n=50$ ) (Fig. 4.12I). Spike 0 also had the highest $\mathrm{dV} / \mathrm{dt}$ maximum in comparison to adjacent Spike - 1 or Spike +1 , confirming the observation in Figure 4.12G $(\mathrm{dV} / \mathrm{dt}$ maxima: Spike -1 median(IQR) $=$ 34.50(31.50-37.43) V/s, Spike $0=45.90(45.04-46.79)$ V/s, Spike $+1=40.37(39.40-$ 41.15) V/s; Spike 0 vs. Spike -1 p $<0.0001$, Spike 0 vs. Spike +1 p $<0.0001$, Wilcoxon ranksum test; $\mathrm{n}=50$ ) (Fig. 4.12J). Taken together, rebound bursting of in vivo midbrain DA neurons is characterized by pronounced hyperpolarizations leading to temporarilyhyperpolarized action potential thresholds and faster rates of depolarization culminating in the initiation of phasic firing. Interestingly, many spikes in this example neuron that would be considered bursts based on this subthreshold membrane signature did not pass the classical burst detection criteria (Spike 0 to Spike +1 ISI median(IQR) = 89.05 (76.87111.4) $\mathrm{ms} ; \% \mathrm{SFB}=36.07 \%$ ) (Grace and Bunney, 1984b), which demonstrates the importance of subthreshold information for functional studies of phasic firing in DA neurons.

Figure 4.12. Rebound bursting of dopamine neurons exhibits hyperpolarized membrane potentials that signal the start of burst. A) Scatterplots of $\log _{10}$ ISI against $V_{\min }$ (blue) and $V_{\text {thr }}$ (green). Clearlysegregated clusters are seen in both plots, but only ISI- $\mathrm{V}_{\min }$ showing separation in both ISI and $\mathrm{V}_{\text {min }}$ axes was further analyzed. Pink mark indicates the centroid of ISI- $V_{\min }$ Cluster 1 , which corresponds to $V_{\min }$ Mode 1 in Fig. 4.9C. B) Snippet of ISI- $V_{\min }$ in (A) was plotted as a continuous line, which reveals a clear trend of ISI- $\mathrm{V}_{\min }$. C) 10 traces containing $\mathrm{V}_{\min }$ from ISI- $\mathrm{V}_{\min }$ Cluster 1 were superimposed at the succeeding spikes (arrow) to reveal stereotypical subthreshold membrane behavior surrounding Cluster 1. D) Mean and SD of (C) illustrates the pronounced hyperpolarization that precedes higher frequency spikes. Blue arrow indicates $V_{\min }$ and blue circle $V_{t h r}$. E) Example three-spike sequence further analyzed. F) Plot showing spikes from 50 three-spike sequences superimposed and aligned at the time of $V_{t h r}$, which demonstrates that Spike -1, Spike 0 and Spike +1 have distinct spike waveforms. $(+)$ symbol indicates $V_{\text {thr }}$ and $(x)$ symbol max. $d V / d t$. G) Averaged traces of ( $F$ ) normalized to $V_{\text {thr }}$ shows the spike amplitude is smallest during Spike -1 and largest during Spike $0 . \mathrm{H}$ ) Phase plot showing the change in rate of depolarization versus membrane potentials. $V_{\text {thr }}$ is lowest and max. $d V / d t$ is highest during Spike $0 . \mathrm{I}$ ) and J) Plots of $\mathrm{V}_{\text {thr }}$ of 50 three-spike sequences (I) and change in max. $\mathrm{dV} / \mathrm{dt}(\mathrm{J})$ show that Spike -1 has a significantly higher $V_{\text {thr }}$ and lowest max. $d V / d t$, while Spike 0 exhibits the opposite phenotype. 
A

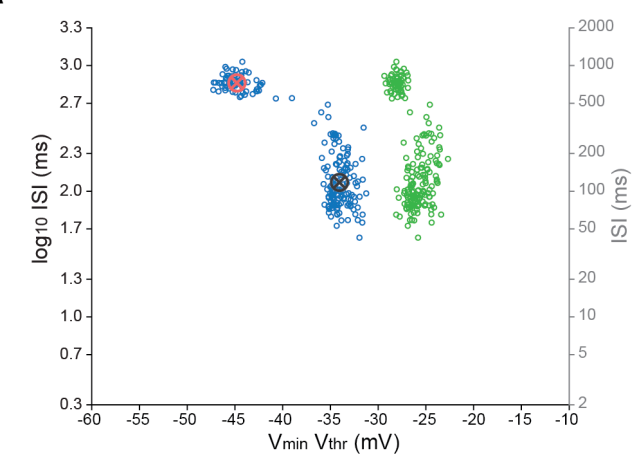

C

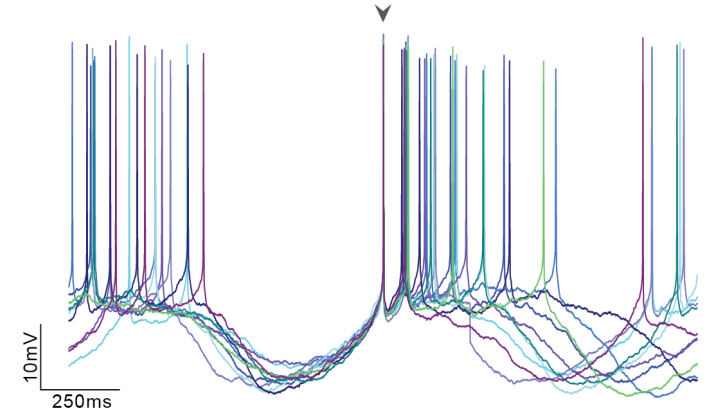

E

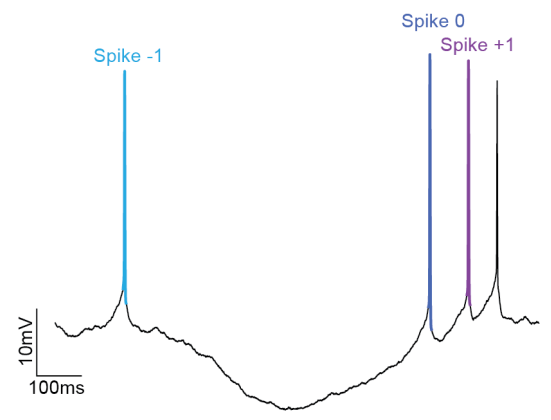

H

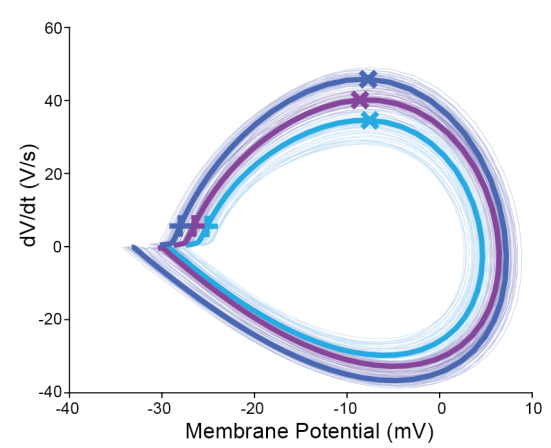

B

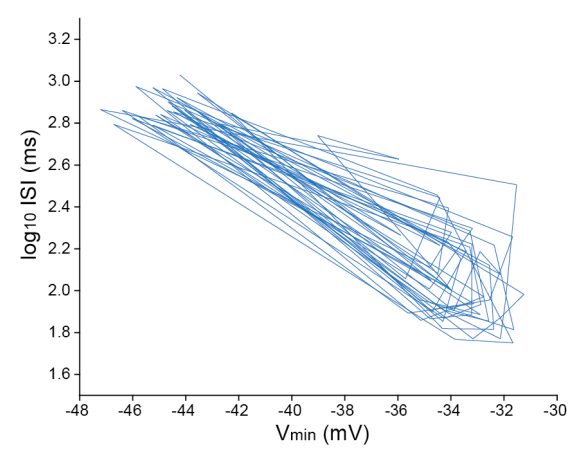

D

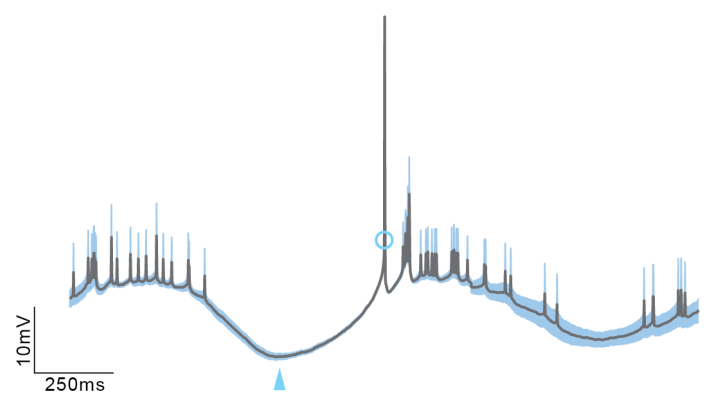

G

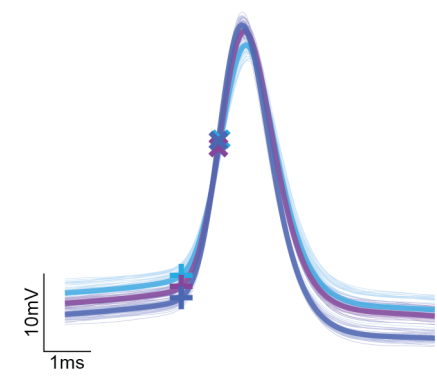

I

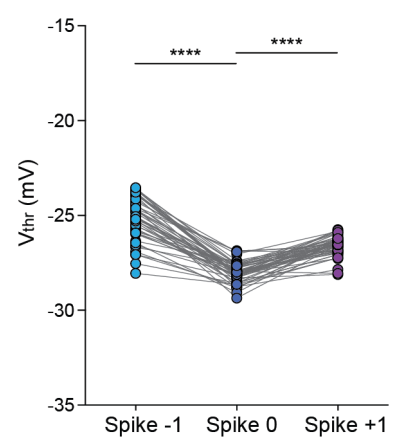

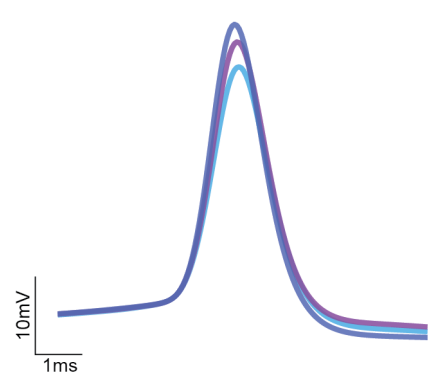

J

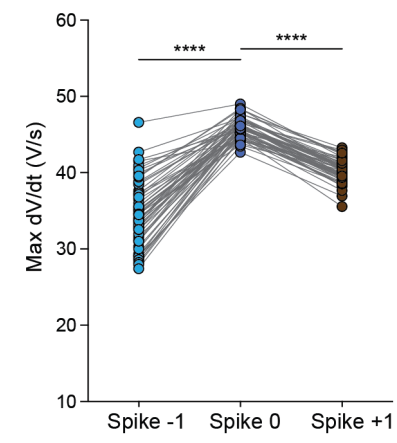


For plateau bursting, plotting $\mathrm{V}_{\min }$ against their corresponding ISI revealed a strong correlation and formed two broadly-distributed clusters (Fig. 4.13A, blue), with a small dense cluster at the shorter ISI and more depolarized $V_{\min }$ range, invading into the voltage range of $\mathrm{V}_{\text {thr. }}$. The ISI versus $\mathrm{V}_{\text {thr }}$ scatterplot also displayed a strong non-linear correlation and created two clusters (Fig. 4.13A, green), with a larger and broader Cluster 1 (black centroid) and a smaller dense Cluster 2 at the bottom right (pink centroid). There appeared to be a pattern in this type of firing, and ISI- $V_{\text {thr }}$ Cluster 1 spikes and Cluster 2 spikes almost always occurred in sequence, as revealed by the continuous ISI- $\mathrm{V}_{\text {thr }}$ line plot (Fig. 4.13B). Based on this observation, ISI-V thr Cluster 2 spikes were aligned at the time of action potential threshold to expose the stereotypical membrane potential behavior from this cluster (Fig. 4.13C). Note that the temporal resolution is 10-fold higher here as compared to pacemaker and rebound burst counterparts. The averaged trace demonstrates the strikingly low-variability subthreshold membrane activity starting from the onset of the preceding spike to the end of the plateau (Fig. 4.13D). Next, to examine the dynamic changes in action potential kinetics of plateau bursting, ISI- $\mathrm{V}_{\text {thr }}$ Cluster 2 spikes and their preceding and succeeding spikes were plotted as before (Fig. $4.13 \mathrm{E})$. Figure $4.13 \mathrm{~F}$ depicts the drastic change in the spike waveform during the threespike sequence, in which the action potential threshold is much more depolarized for Spike 0 as compared to Spike -1 or Spike +1 . Normalizing these average traces to the spike threshold voltage uncovered the striking reduction in the Spike 0 amplitude and the Spike 0 AHP which continued downward as opposed to the Spike -1 and Spike +1 AHP which quickly began to depolarize membrane potentials following repolarization (Fig.4.13G). Interestingly, the waveforms of Spike -1 and Spike +1 were almost identical, apart from the slightly reduced amplitude in Spike -1 . The drastic difference of Spike 0 was also evident in the phase plot which showed Spike 0 having much slower action potential kinetics as compared to the preceding and following spikes (Fig. 4.13H). Figure 4.13I demonstrates that, during plateau bursting, action potential thresholds for Spike 0 became significantly depolarized as compared to Spike -1, but recovered in the subsequent Spike $+1\left(\mathrm{~V}_{\text {thr: }}\right.$ Spike -1 median $(I Q R)=-33.55(-34.06$ to -31.67$) \mathrm{mV}$, Spike 0 $=-27.39(-27.90$ to -26.32$) \mathrm{mV}$, Spike $+1=-34.16(-34.40$ to -33.75$) \mathrm{mV}$; Spike 0 vs. Spike $-1 \mathrm{p}<0.0001$, Spike 0 vs. Spike $+1 \mathrm{p}<0.0001$, Wilcoxon rank-sum test; $\mathrm{n}=50$ ). 
Concurrent with the rise in spike thresholds is the significantly reduced rate of change in membrane potentials in general but particularly noticeable at $\mathrm{dV} / \mathrm{dt}$ maxima, which also recovered in the next Spike $+1(\mathrm{dV} / \mathrm{dt}$ maxima: Spike -1 median $(\mathrm{IQR})=48.77(41.72$ 50.27) V/s, Spike $0=29.85(25.26-31.48)$ V/s, Spike $+1=50.43(49.28-51.65)$ V/s; Spike 0 vs. Spike $-1 \mathrm{p}<0.0001$, Spike 0 vs. Spike $+1 \mathrm{p}<0.0001$, Wilcoxon rank-sum test; $\mathrm{n}=50$ )

(Fig.4.13J). Based on the results, plateau bursting is defined by the transiently depolarized plateau potentials where high-frequency spikes result in more depolarized action potential thresholds and slower rates of depolarization, culminating in the termination of phasic firing.

Figure 4.13. Plateau bursting of dopamine neurons shows plateau membrane potentials where burst terminates. A) Scatterplots of $\log _{10} I S I$ against $V_{\text {min }}$ (blue) and $V_{\text {thr }}$ (green). Spread-out clusters are observed in both plots. Centroid of ISI- $\mathrm{V}_{\text {thr }}$ Cluster 2 is shown in pink, which corresponds to $\mathrm{V}_{\text {thr }}$ Mode 2 in Fig. 4.10C. B) Snippet of ISI- $V_{\text {thr }}$ in (A) was plotted as a continuous line, which shows a pattern similar to $4.12 \mathrm{~B}$. C) 10 traces containing $\mathrm{V}_{\text {thr }}$ from ISI- $\mathrm{V}_{\text {thr }}$ Cluster 2 were superimposed at the corresponding spikes (arrow) to see stereotypical subthreshold membrane behavior surrounding Cluster 2. Note the higher (10x) resolution of traces in comparison to 4.11C and 4.12C. D) Mean and SD of $(C)$ reveals the two action potentials firing at precise intervals riding on top of transient plateau potentials. Blue circles indicate $V_{\text {thr. }}$ E) Example three-spike sequence further analyzed. F) Plot showing spikes from 50 three-spike sequences superimposed and aligned at the time of $V_{\text {thr. }}$. Spike 1 , Spike 0 and Spike +1 all differ in shape of spike waveforms. (+) symbol indicates $V_{\text {thr }}$ and $(x)$ symbol max. $d V / d t$. G) Averaged traces of (F) normalized to $V_{\text {thr }}$ voltage shows the spike amplitude is notably small during Spike 0 as compared to Spike -1 or Spike +1 . H) Phase plot highlights the contrast between Spike 0 and Spike $-1 /+1$, where $V_{\text {thr }}$ is highest and max. $d V / d t$ is lowest during Spike 0.1 ) and J) Plots of $V_{\text {thr }}$ of 50 three-spike sequences $(\mathrm{I})$ and change in max. $\mathrm{dV} / \mathrm{dt}(\mathrm{J})$ show that Spike 0 has a significantly higher $\mathrm{V}_{\text {thr }}$ and lowest max. $\mathrm{dV} / \mathrm{dt}$. 
A

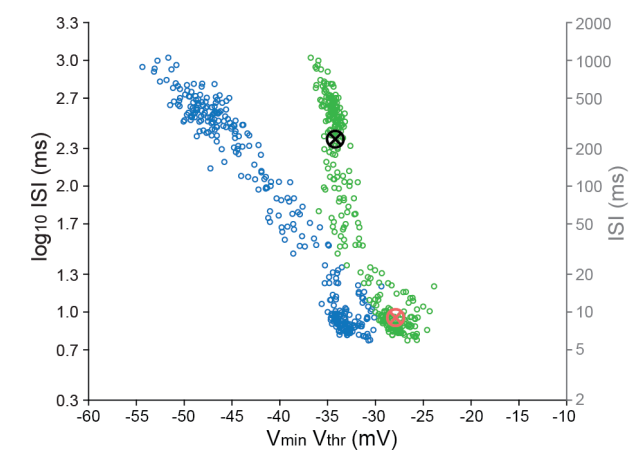

C

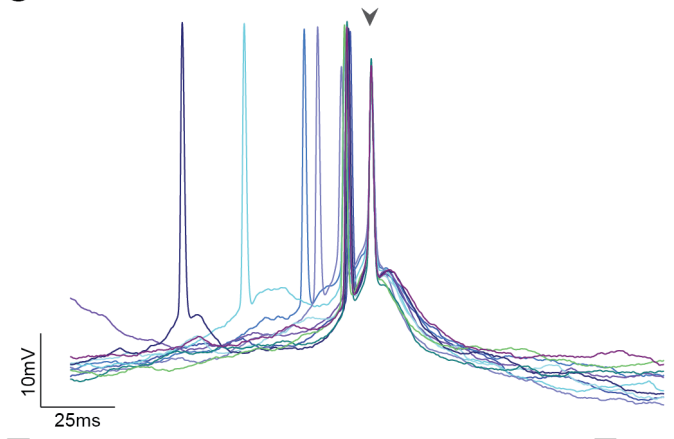

E

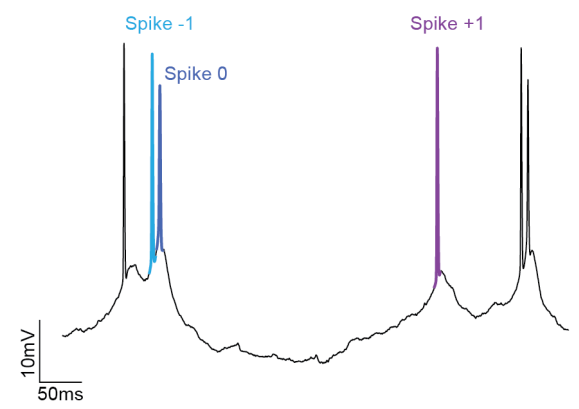

H

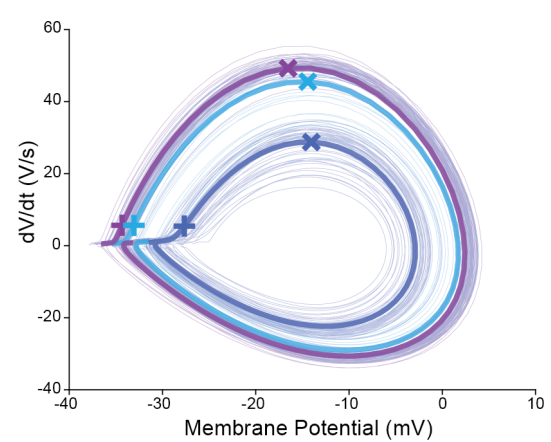

B

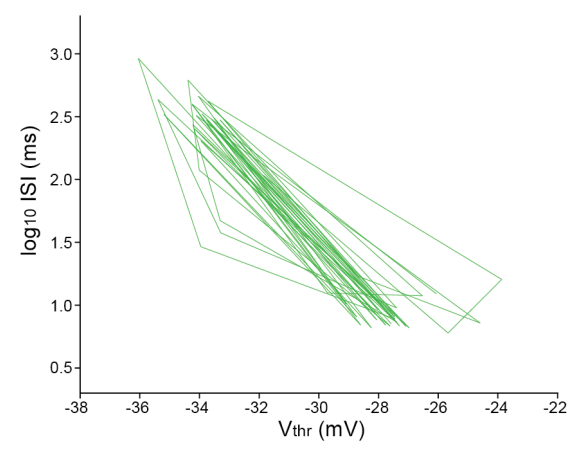

D

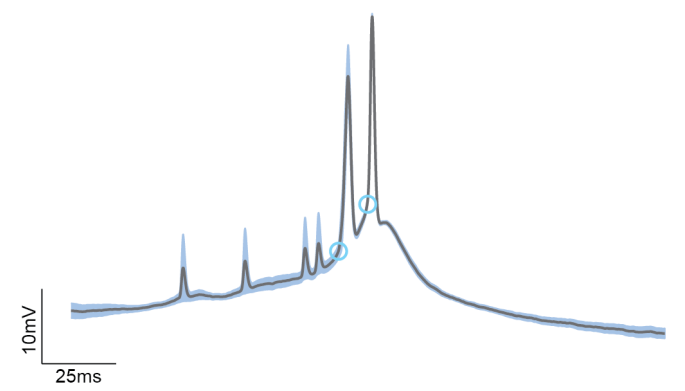

G

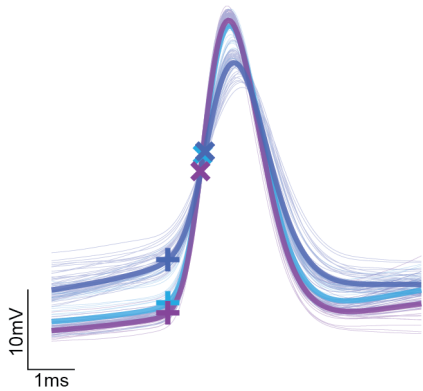

I

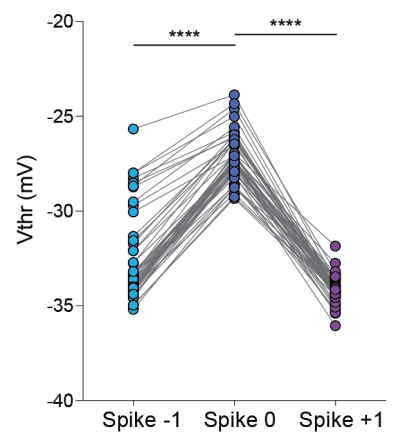

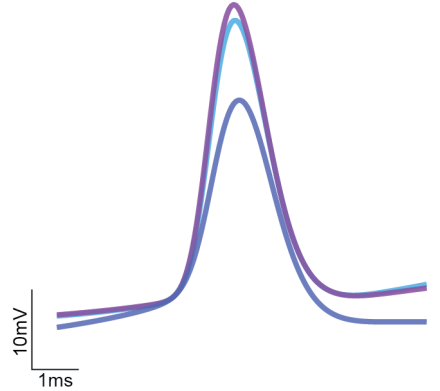

J

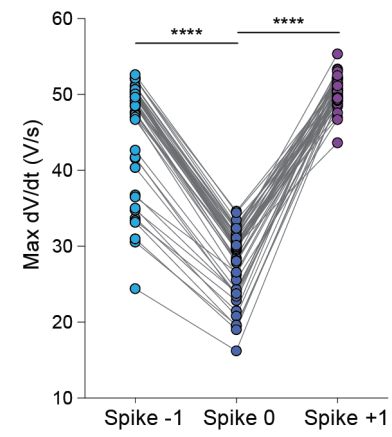


DA neurons can also display a combination of rebound and plateau bursts, and a few recorded neurons indeed showed such activity (Fig. 4.14A). This multipolar neuron was in the dorsolateral portion of the VTA (Fig. 4.14B). $\mathrm{V}_{\text {min }}$, marked as blue circles, and $\mathrm{V}_{\text {thr, }}$ in green circles, exhibited activity resembling both rebound bursting and plateau bursting described earlier; with pronounced hyperpolarizations and high-frequency bursts on top of transient depolarized plateau potentials, which are riding on a wave of slower depolarization (Fig. 4.14A, bottom). The $\mathrm{V}_{\min }$ distribution displays a clear trimodal shape with a broad range (Fig. 4.14C), whereas the $\mathrm{V}_{\text {thr }}$ distribution shows a more complicated multimodal identity in a narrow $\mathrm{V}_{\text {thr }}$ range (Fig. 4.14D). The three levels of $V_{\text {min }}$ and $V_{\text {thr }}$ can be closely examined in the example trace with a high temporal resolution (Fig. 4.14E). The scatterplots of ISI versus respective $V_{\min }$ and $V_{\text {thr }}$ show a hybrid phenotype of rebound burst and plateau burst modes, forming three clusters each (Fig. 4.14F). This example suggests that DA neurons embedded in circuits may use combinations of single spiking, rebound bursting, and/or plateau bursting to convey their complex signals. 
A
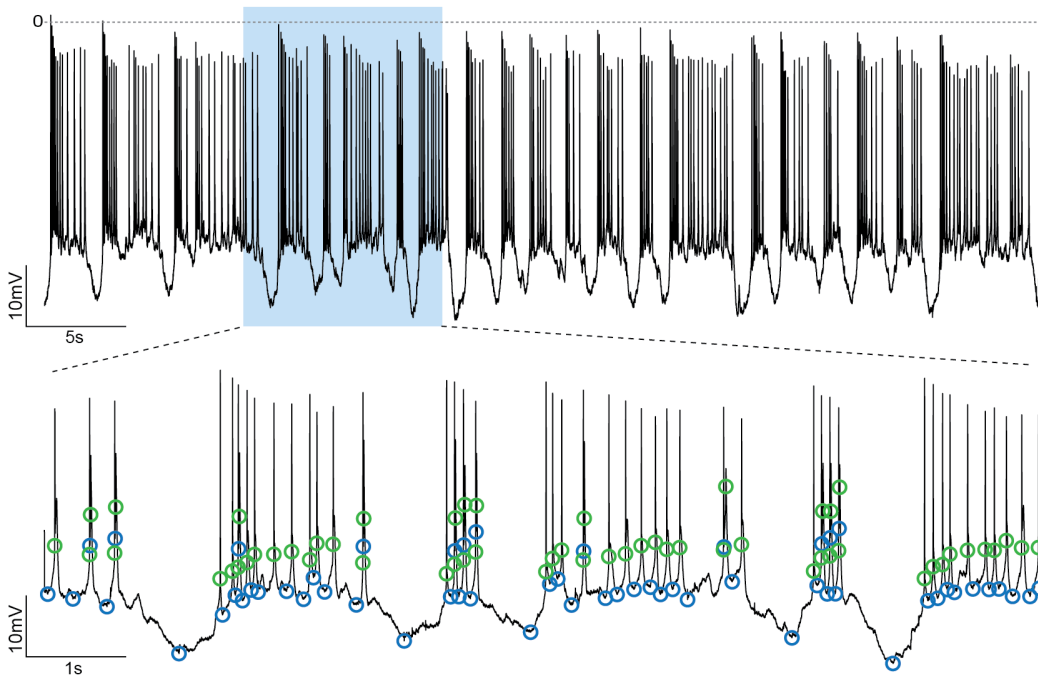

C

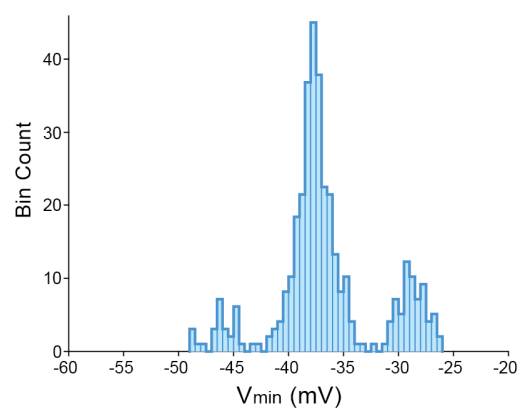

E

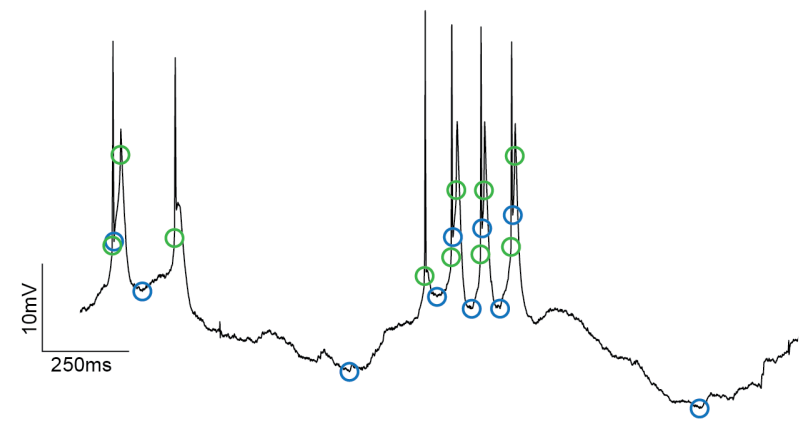

B

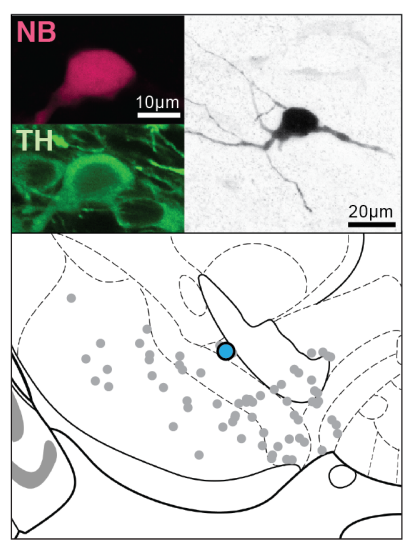

D

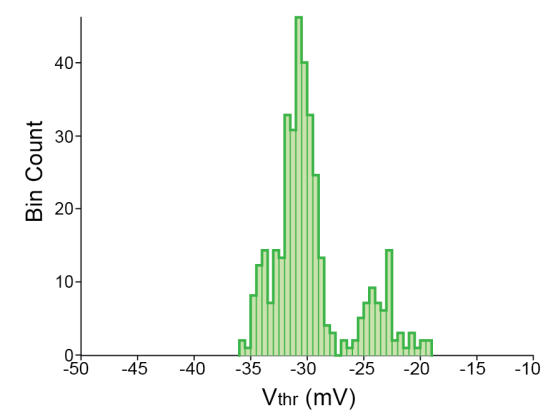

F

Figure 4.14. Dopamine neurons can exhibit a combination of rebound and plateau bursting. A) Top: Example 50 -second trace of a DA neuron with trimodal $V_{\min }$ and bimodal $V_{\text {thr }}$ distributions. Bottom: 10-second snippet of the top trace. $V_{\min }$ is in blue circles and $V_{\text {thr }}$ in green circles. Both $V_{\min }$ and $V_{\text {thr }}$ are constantly fluctuating at different membrane potential levels. B) Top: Histology of the neuron in (A). Bottom: Location of this neuron was in the VTA. C) Histogram of $V_{\min }$ shows a trimodal distribution. D) Histogram of $V_{\text {thr }}$ displays a clear bimodal distribution. E) Example trace in higher resolution to present details of $\mathrm{V}_{\min }$ and $\mathrm{V}_{\text {thr }}$ fluctuations. F) ISI- $\mathrm{V}_{\min }$ and ISI- $\mathrm{V}_{\text {thr }}$ scatterplots show clusters resembling Figure 4.12A and 4.13A combined. This group of dopamine neurons is considered rebound-plateau bursters. Brain illustration adapted with permission from G. Paxinos and K. Franklin, 2007. Copyright (2007) Elsevier. 


\subsection{Characterization of subthreshold membrane behaviors of the population}

The preceding sections have highlighted the broad spectrum of subthreshold membrane behaviors of in vivo midbrain DA neurons that are the basis of their unique firing patterns, using representative neurons. However, a means to describe the distinct subthreshold membrane signatures of the entire recorded population is missing. This may become important when attempting to demonstrate subthreshold-based firing trends in different experimental groups, for example. To proceed, I wanted to first classify all DA neurons in my dataset according to $V_{\min }$ and $V_{\text {thr }}$ distributions. This however proved tricky, because while many DA neurons with clear unimodal or bimodal $V_{\min } / V_{\text {thr }}$ distributions could be easily classified, some neurons with fewer burst events or irregular activity were more difficult to categorize, as weak bimodal distributions could not be distinguished from non-Gaussian skewness. According to literature, the best available methods for bimodality detection are bimodality coefficients (BC), Hartigan's dip statistics (HDS), and the combination of the two (Freeman and Dale, 2013; Kang and Noh, 2019). However, only a few neurons in which the distributions were clearly bimodal could be identified by these methods using the established $\mathrm{BC}>0.555$ for bimodality detection and HDS $<0.05$ for unimodality detection. This is presumably because greater sample size is required to accurately detect weak or complex multimodalities using these methods (Freeman and Dale, 2013; Kang and Noh, 2019). Therefore, I used kernel density estimation (KDE) to first estimate the distributions of $V_{\min }$ and $V_{t h r}$ and extracted the number of modes from each distribution based on density derivative estimation, i.e. first derivative of KDE (Chacón and Duong, 2020). For consistency, the bandwidth of 0.65 was used for all dataset, which was manually tested and determined to be most accurate against automatically-selected bandwidths via bandwidth selectors. This KDE-based method accurately identified $V_{\min }$ and $V_{\text {thr }}$ distributions and thus could classify all recorded in vivo DA neurons into distinct firing patterns, which revealed that 40 neurons fired in regular or irregular single-spiking patterns, 21 neurons exhibited rebound bursts, 2 neurons had plateau bursts and 4 neurons showed both rebound and plateau bursting. The parameters directly or indirectly related to subthreshold characteristics, CV, SD of $V_{\text {min }}, S D$ of $V_{\text {thr }}, B C$ of $V_{\text {min }}$ and $B C$ of $V_{\text {thr }}$, were plotted for comparison, which showed that 
A

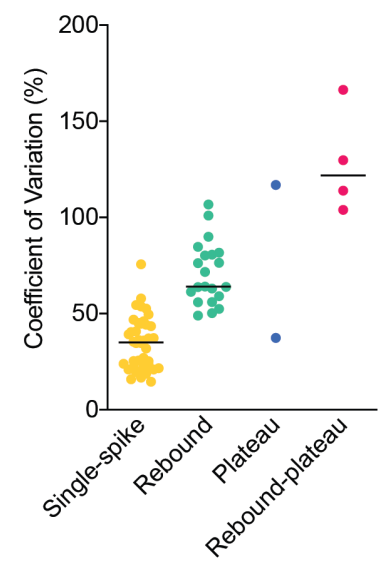

D

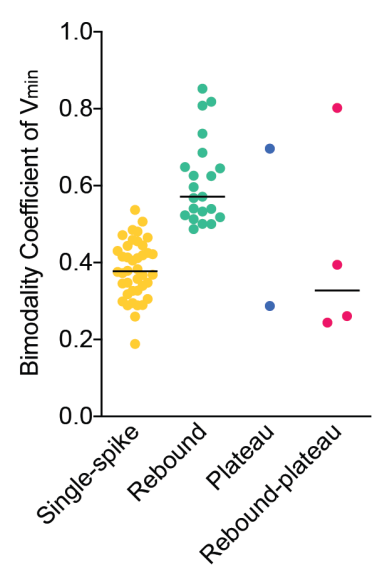

$\mathbf{F}$

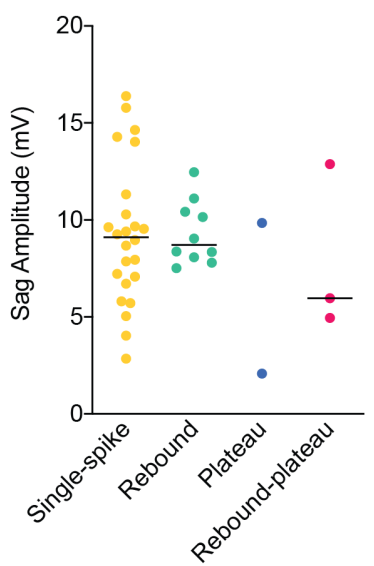

G
B

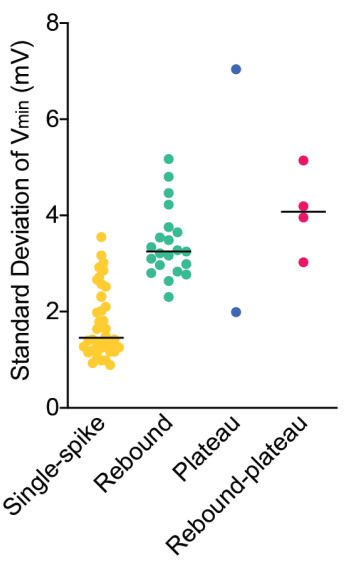

C

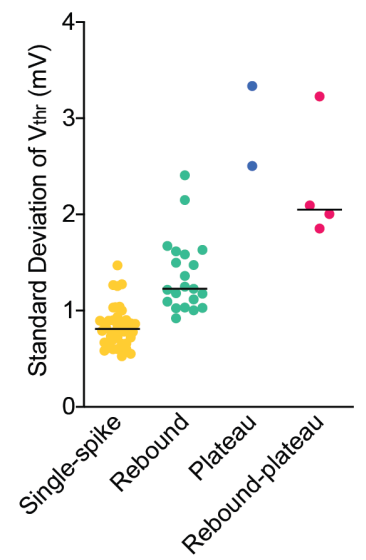

E

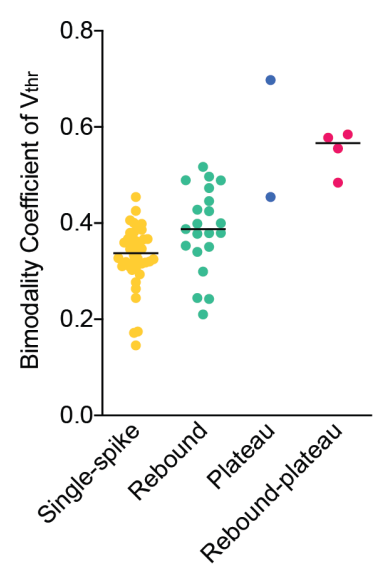

H
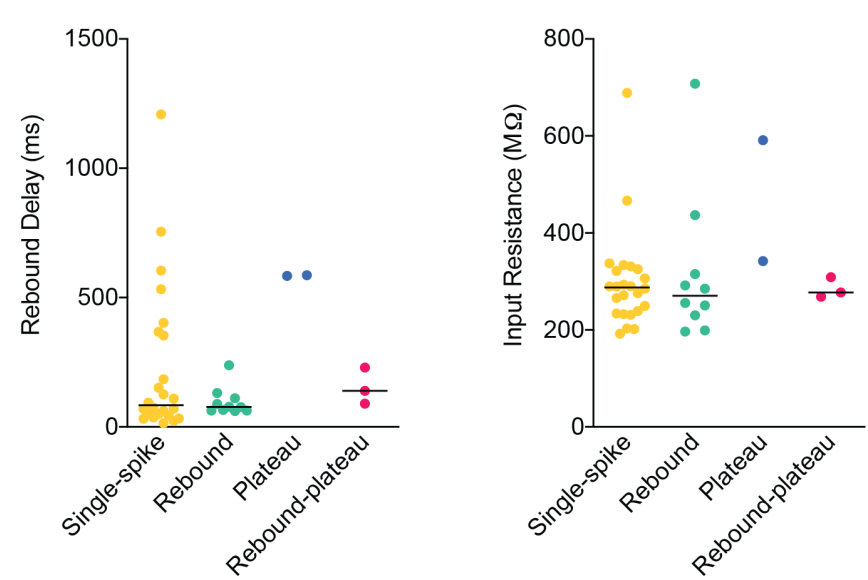

Figure 4.15. Properties of distinct subthreshold-based firing patterns identified by KDE derivative. A) Plot of coefficient of variation, which is an indicator of variabilities in ISI, differentiates subthreshold membrane activity-based firing patterns to some level. B) Standard deviation of $V_{\min }$. C) Standard deviation of $V_{\text {thr. }}$. D) Bimodality coefficient of $V_{\text {min }}$. E) Bimodality coefficient of $V_{\text {thr. }}$ F) Sag amplitude. Rebound bursting dopamine neurons have large sag. G) Rebound delay. Plateau bursting dopamine neurons display long rebound delay. $\mathrm{H}$ ) Input resistance. 
all of these values discriminated subthreshold-based firing patterns to some degree, as expected (Fig. 4.15A to 4.14E). Of particular interest was the CV plot, which suggested that prediction of underlying subthreshold behavior of phasic firing might be possible for extracellularly-recorded neurons with a CV of approximately $60 \%$ or above, where CV greater than $100 \%$ would hint at the presence of plateau bursts (Fig. 4.15A). Furthermore, additional details of the four classes were uncovered by plotting intrinsic membrane properties according to subthreshold membrane-based firing patterns (Fig. $4.15 \mathrm{~F}-4.15 \mathrm{H})$. Based on the three burst classes alone, rebound bursting occurs only in DA neurons that display a large sag amplitude, while plateau bursting is observed in DA neurons that exhibit a long rebound delay. Although the sample size is small, this could indicate that, while DA neurons may alter firing patterns between single spiking and bursting, the presence of $I_{H}$ and $I_{A}$ may determine the type of bursting DA neurons are capable of, meaning that these distinct subthreshold burst signatures may be limited to certain DA subpopulations. Median and interquartile range for each plot is summarized in Table 4.1.

Table 4.1. Summary of median(IQR) values for each class of subthreshold membrane activity-based firing patterns.

\begin{tabular}{|c|c|c|c|c|}
\hline & $\begin{array}{c}\text { Single-spike } \\
\mathrm{n}=40,24^{*}\end{array}$ & $\begin{array}{l}\text { Rebound burst } \\
\mathrm{n}=21,10^{*}\end{array}$ & $\begin{array}{c}\text { Plateau burst } \\
\mathrm{n}=2,2^{*}\end{array}$ & $\begin{array}{c}\text { Rebound-plateau } \\
n=4,3^{*}\end{array}$ \\
\hline CV (\%) & $\begin{array}{c}35.01 \\
(22.29-44.59)\end{array}$ & $\begin{array}{c}64.10 \\
(57.54-81.16)\end{array}$ & $\begin{array}{c}77.16 \\
(37.40-116.9)\end{array}$ & $\begin{array}{c}121.8 \\
(106.4-157.2)\end{array}$ \\
\hline$S D V_{\min }(m V)$ & $\begin{array}{c}1.46 \\
(1.21-2.32)\end{array}$ & $\begin{array}{c}3.25 \\
(2.90-3.70)\end{array}$ & $\begin{array}{c}4.52 \\
(1.99-7.04)\end{array}$ & $\begin{array}{c}4.08 \\
(3.26-4.91)\end{array}$ \\
\hline$S D V_{\text {thr }}(m V)$ & $\begin{array}{c}0.811 \\
(0.660-1.901)\end{array}$ & $\begin{array}{c}1.23 \\
(1.06-1.60)\end{array}$ & $\begin{array}{c}2.92 \\
(2.50-3.34)\end{array}$ & $\begin{array}{c}2.05 \\
(1.89-2.94)\end{array}$ \\
\hline$B C V_{\text {min }}$ & $\begin{array}{c}0.377 \\
(0.327-0.44)\end{array}$ & $\begin{array}{c}0.572 \\
(0.520-0.667)\end{array}$ & $\begin{array}{c}0.492 \\
(0.288-0.696)\end{array}$ & $\begin{array}{c}0.248 \\
(0.328-0.700)\end{array}$ \\
\hline BC $V_{\text {thr }}$ & $\begin{array}{c}0.338 \\
(0.309-0.377)\end{array}$ & $\begin{array}{c}0.388 \\
(0.346-0.460)\end{array}$ & $\begin{array}{c}0.576 \\
(0.454-0.698)\end{array}$ & $\begin{array}{c}0.567 \\
(0.502-0.583)\end{array}$ \\
\hline $\begin{array}{c}\text { Sag Amplitude } \\
(\mathrm{mV})\end{array}$ & $\begin{array}{c}9.11 \\
(6.80-11.1)\end{array}$ & $\begin{array}{c}8.71 \\
(8.00-10.6)\end{array}$ & $\begin{array}{c}5.96 \\
(2.08-9.84)\end{array}$ & $\begin{array}{c}5.97 \\
(4.95-12.9)\end{array}$ \\
\hline $\begin{array}{l}\text { Rebound Delay } \\
\text { (ms) }\end{array}$ & $\begin{array}{c}84.3 \\
(45.4-363.7)\end{array}$ & $\begin{array}{c}77.1 \\
(63.1-116.1)\end{array}$ & $\begin{array}{c}584.9 \\
(583.7-586.2)\end{array}$ & $\begin{array}{c}139.7 \\
(89.7-229.6)\end{array}$ \\
\hline $\begin{array}{c}\text { Input Resistance } \\
\text { (M } \Omega)\end{array}$ & $\begin{array}{c}287.2 \\
(235.2-324.5)\end{array}$ & $\begin{array}{c}270.3 \\
(222.2-345.5)\end{array}$ & $\begin{array}{c}466.5 \\
(341.8-591.1)\end{array}$ & $\begin{array}{c}277.3 \\
(268.3-308.6)\end{array}$ \\
\hline
\end{tabular}


(*) symbol next to a number indicates the number of neurons plotted for sag amplitude, rebound delay, and input resistance. BC: bimodality coefficient; CV: coefficient of variation; IQR: interquartile range; SD: standard deviation; $\mathrm{V}_{\min }$ : subthreshold membrane minimum; $\mathrm{V}_{\text {thr }}$ : action potential threshold.

In order to present the subthreshold activity-based firing properties of in vivo midbrain DA neurons as a population, I first mapped the four group's anatomical distributions, shown in Figure 4.16A. Single-spiking neurons and rebound bursters cover most of the DA-rich midbrain region, while plateau bursters and rebound-plateau bursting neurons are seen in the medial VTA and more dorsomedial SNc/dorsolateral VTA parts, respectively, though no conclusion should be drawn due to the small sample size for the latter subpopulations. Next, since subthreshold membrane activity and related firing patterns are in fact continuous rather than discrete, I plotted $\mathrm{BC}$ of $\mathrm{V}_{\text {min }}$ against $\mathrm{BC}$ of $\mathrm{V}_{\text {thr }}$ in a logarithmic scale to create a two-dimensional (2D) graph of subthreshold-based firing pattern distribution (Fig. 4.16B). While BC greater than 0.555 $\left(\log _{10}\right.$ of which is -0.26$)$ was not reliable in identifying all phasic firing neurons in my dataset, the $\mathrm{BC}$ plot created well-segregated groups of distinct firing pattern in this 2D space, with pacemaking/single-spiking neurons in the bottom left quadrant, rebound burst neurons in the top left quadrant, one plateau bursting neuron in the top right quadrant and rebound-plateau burst neurons in the bottom right quadrant. Here, reference lines separating the quadrants were drawn at $-0.30\left(=\log _{10} 0.5\right)$ rather than $0.26\left(=\log _{10} 0.555\right)$ on both axes which better accommodated phasic firing neurons within the borders, with a few contaminations along these lines. SD of $\mathrm{V}_{\text {min }}$ versus SD of $\mathrm{V}_{\text {thr }}$ was also plotted for comparison but resulted in a more linear distribution with much more overlap between groups, making it less useful than the BC plot. Thus, whereas the anatomical map provides the topographical organization of in vivo DA subpopulations linked to distinct firing patterns, the $V_{\min } B C$ versus $V_{\text {thr }} B C$ plot can be used to characterize the distribution of subthreshold membrane activity-based firing patterns of in vivo DA neurons as a population, which will allow comparison of different populations. 
A

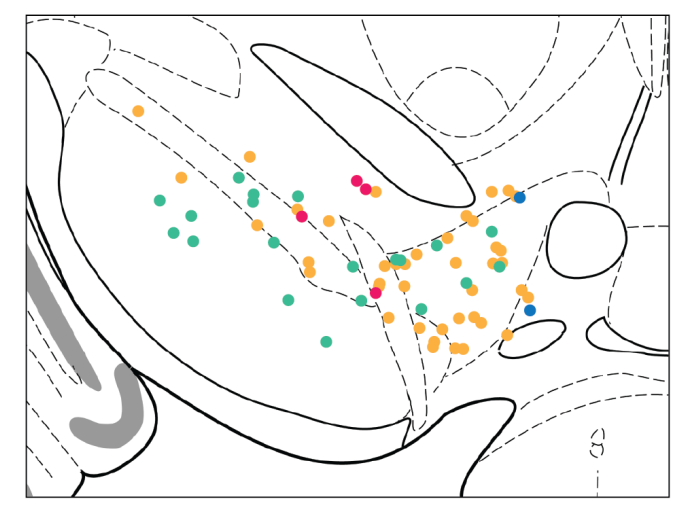

B

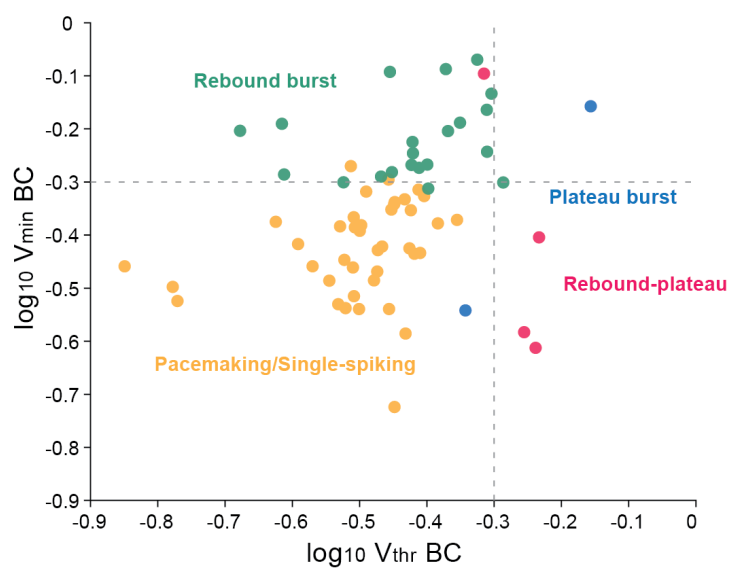

Figure 4.16. Distribution of subthreshold membrane behavior-based firing patterns. A) Anatomical map of subthreshold-based firing patterns. Pacemaker and rebound bursters were spread out, while plateau and rebound-plateau bursters may have preferential anatomical location. B) $V_{\min } B C$ and $V_{\text {thr }}$ $B C$ plot creates four quadrants where dopamine neurons with distinct subthreshold-based firing patterns preferentially occupy. Dotted lines indicate $B C \approx 0.5$. Brain illustration adapted with permission from G. Paxinos and K. Franklin, 2007. Copyright (2007) Elsevier. 


\section{ADDITIONAL RESULTS}

\subsection{Subthreshold membrane activity and circadian rhythm}

There is now a consensus that a link exists between the circadian system and the reward system (Albrecht, 2020; Verwey et al., 2016; Webb et al., 2015). Multiple "clock" genes that play main roles in circadian rhythms regulate the expression of molecules involved in DA metabolism in the mesolimbic system, such as TH and MAO-A (Chung et al., 2014; Hampp et al., 2008; Hood et al., 2010). At the same time, DA in turn affects the circadian system by controlling the expression of a clock protein PER2 via striatal D2R (Hood et al., 2010). In schizophrenia, there is a bidirectional relationship between the DA system and circadian rhythm, and perturbation to one system can cause disruption in the other system (Ashton and Jagannath, 2020). A recent study in mice found that a mutated Clock gene increased firing rate in VTA DA neurons during daytime when mice are inactive, which led to manic-like behavior (Sidor et al., 2015).

Considering my main findings, it is plausible that the increased firing rate in DA neurons is due to changes in their subthreshold membrane patterns. As a first step to elucidating how intrinsic and extrinsic mechanisms shape baseline DA signaling during the course of day, I examined the subthreshold membrane activity of DA neurons during circadian rhythms. The same dataset from the previous section was therefore reanalyzed to study the subthreshold basis of in vivo DA neuron firing patterns in a circadian-relevant manner. Recordings were divided into two groups: 1) the Light Cycle (LC) group consisting of 23 recordings $(\mathrm{N}=20)$ obtained from 8:00am to 8:00pm, and 2) the Dark Cycle (DC) group consisting 44 recordings ( $\mathrm{N}=33$ ) obtained from 8:00pm to 8:00am (adjusted for daylight saving time).

Results show that, although there was no significant difference in the firing rate between LC and DC, CV was significantly higher in DC as compared to LC (LC FR mean \pm SD $=4.58 \pm 1.96 \mathrm{~Hz} ; \mathrm{p}=0.657$, unpaired t-test; LC median(IQR) = 36.01(21.59-53.73) \%; DC median $(I Q R)=52.55(36.47-74.67) \% ; p=0.0262$, Mann-Whitney U test) (Fig. 5.1A and 5.1B). As predicted, the increase in $C V$ was accompanied by changes in $S D$ of $V_{\text {min }}$ and $V_{t h r}$, both of which were significantly increased during DC when mice are active (LC SD $V_{\text {min }}$ median $(I Q R)=1.64(1.26-2.66) \mathrm{mV} ; \mathrm{DC}$ SD $\mathrm{V}_{\min }=2.95(1.69-3.53) \mathrm{mV} ; \mathrm{p}=0.0039$, Mann- 
Whitney U test; LC SD V thr median(IQR) $=0.86(0.63-1.04) \mathrm{mV}$; DC SD $\mathrm{V}_{\text {thr }}=1.03(0.81$ 1.47) $\mathrm{mV} ; \mathrm{p}=0.0449$, Mann-Whitney U test) (Fig. 5.1C and 5.1D). Furthermore, mapping the two populations in the $\mathrm{BC}$ of $\mathrm{V}_{\text {min }}$ versus $\mathrm{BC}$ of $\mathrm{V}_{\text {thr }}$ plot revealed that, while most LC DA neurons were confined to the single-spiking and irregular firing domains, DC DA neurons occupied all quadrants including the phasic firing domains (Fig. 5.1E). KDEbased identification confirmed that $62 \%$ of DC DA neurons were phasic firing, whereas only $21 \%$ of LC DA neurons fired in bursts (LC: single-spike $n=19$, rebound burst $n=3$, plateau burst $n=1$, rebound-plateau $n=0$; DC: single-spike $n=21$, rebound burst $n=8$, plateau burst $n=1$, rebound-plateau $n=4$ ). Strikingly, all rebound-plateau bursting neurons were recorded during DC.

Taken together, midbrain DA neurons have increased subthreshold membrane fluctuations during the night as compared to the day, leading to more burst events during mice's active phase. Further study is needed to determine the mechanism by which DA neurons' subthreshold membrane activity alters during the diurnal cycle and the circuit involved. In addition, it will also be informative to examine how disruption of the circadian rhythm impacts subthreshold activity of DA neurons and the functional consequences of such perturbation.

Figure 5.1. Subthreshold membrane activity of dopamine neurons during the day versus night. Dataset from previous chapter was separated into Light Cycle (LC) and Dark Cycle (DC) groups according to the time of recording (LC: $8 \mathrm{am}-8 \mathrm{pm}$; DC: $8 \mathrm{pm}-8 \mathrm{am}$ ). A) Plot of firing rates in LC versus DC dopamine neurons. While there was no significance, DC group had a higher frequency trend. B) CV was significantly higher during DC. C) and D) Both standard deviations of $V_{\text {min }}(C)$ and $V_{\text {thr }}(D)$ were higher during DC. E) Bimodality coefficient map shows that burst activity is generally higher during DC. 
A

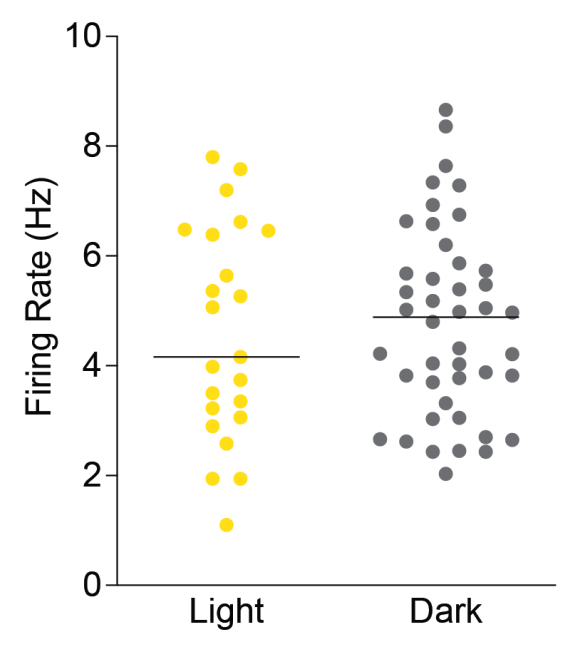

C

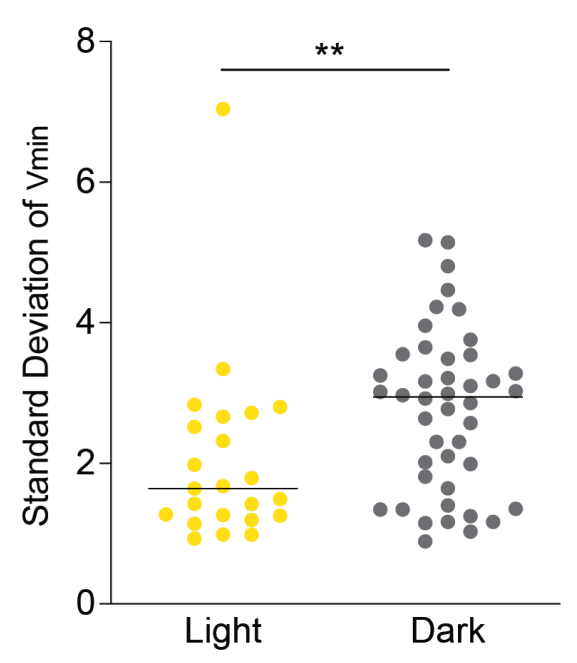

B

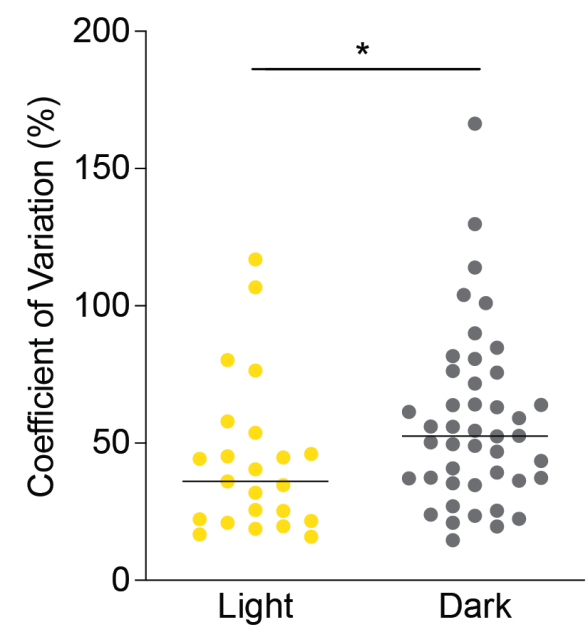

D

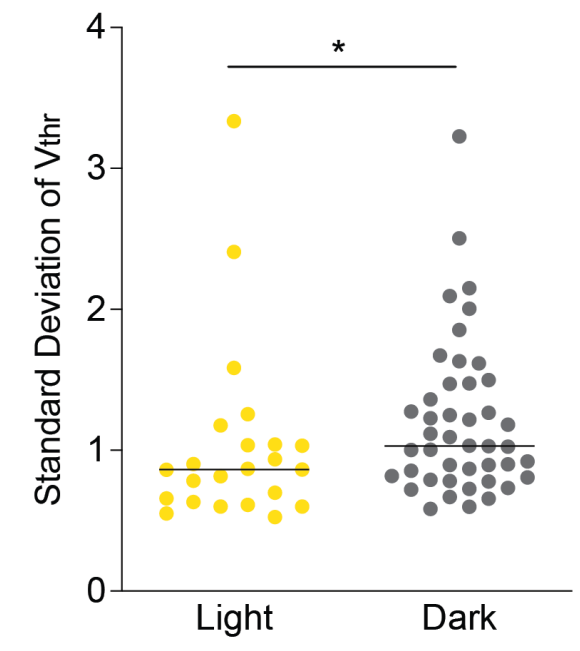

E

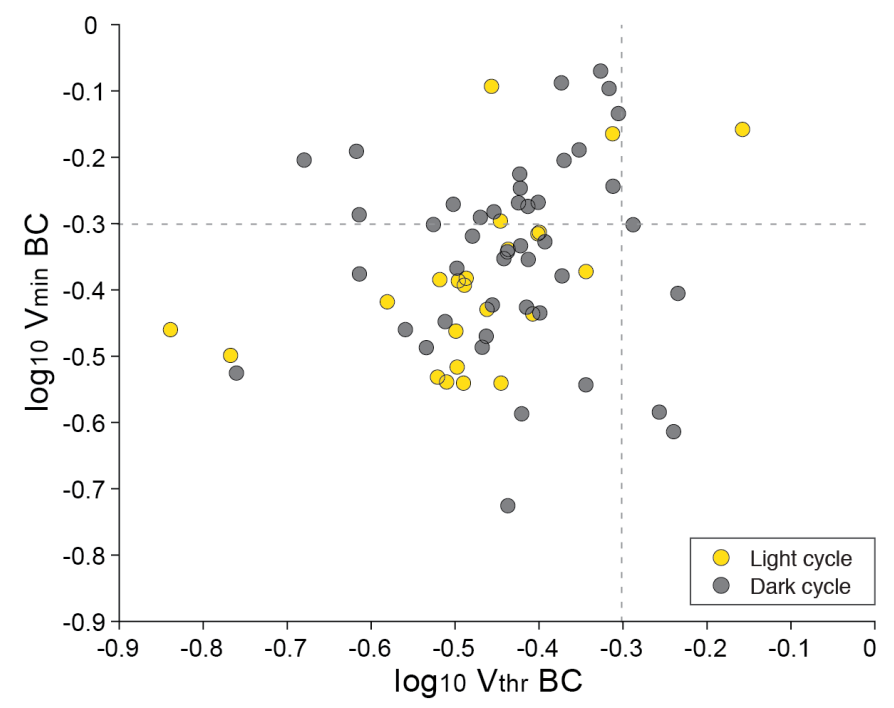




\subsection{Projection-specific subthreshold membrane properties}

Previous work from our lab described distinct electrophysiological properties of projection-defined midbrain DA neurons in vitro (Lammel et al., 2008). A subsequent in vivo study performed on anesthetized mice demonstrated that in vivo firing activity varies in distinct projection-specific DA neurons (Farassat et al., 2019). To further characterize the subthreshold membrane potential dynamics that drive firing of in vivo DA neurons in a projection-defined manner, I carried out in vivo whole-cell patch-clamp recordings combined with retrograde tracing. A retrograde tracer Fluoro-Gold (FG) was infused into mesolimbic DA neurons' main axonal targets, the lateral shell and medial shell of nucleus accumbens (lNAcc: AP $=0.86 \mu \mathrm{m}, \mathrm{ML}= \pm 1.75 \mu \mathrm{m}, \mathrm{DV}=4.5 \mu \mathrm{m} ;$ mNAcc: $\mathrm{AP}=1.54 \mu \mathrm{m}, \mathrm{ML}$ $= \pm 0.7 \mu \mathrm{m}, \mathrm{DV}=4.25 \mu \mathrm{m}$ ) (Fig. 5.2A). The optimal concentration of FG that causes no structural damage or instability in DA neuron firing was determined to be $0.002 \%$ in a previous study (Farassat et al., 2019). In vivo whole-cell recordings were obtained seven to ten days after FG infusion (Fig. 5.2B). Post hoc immunohistochemistry was performed on coronal midbrain sections to fluorescently label TH, FG and NB. FG's intrinsic fluorescence in the striatum was sufficient to determine infusion sites (Fig. 5.2C). Retrogradely-traveled FG in DA neurons was mostly observed in what appeared like vesicles, which were likely lysosomes according to literature (Persson and Havton, 2009; Schmued et al., 1989).

Nine out of 36 recorded neurons were FG-positive and co-localized with TH (25\% success rate), and five neurons were found to project to the INAcc and four to the mNAcc (lNAcc: $\mathrm{n}=5, \mathrm{~N}=4$; mNAcc: $\mathrm{n}=4, \mathrm{~N}=4$; respectively; Fig. 5.2D). Example recordings of INAcc- and mNAcc-projecting DA neurons are shown in Figure 5.2E and 5.2G, respectively, with corresponding histology images in Figure 5.2F and 5.2H. Consistent with past studies, identified DA neurons projecting to the INAcc were located in the more dorsolateral part of the VTA, whereas those projecting to the mNAcc were found in the more ventromedial aspect (Farassat et al., 2019; Lammel et al., 2008). Although statistical significance could not be determined due to the small sample size, the two subpopulations exhibited some trends in their electrophysiological properties. The plot in Figure 5.3A shows the mean firing rates of INAcc- and mNAcc-projecting DA neurons illustrating the potentially higher firing rates in the mNAcc group as compared to INAcc 
(INAcc FR mean \pm SD: $3.89 \pm 1.17 \mathrm{~Hz}$; mNAcc FR mean \pm SD: $4.98 \pm 1.99 \mathrm{~Hz}$ ). This result may be somewhat different from previous results from extracellular recordings of projectionspecific DA neurons which showed similar firing rate ranges for both subpopulations but mNAcc-projecting DA neurons had a significantly lower median (Farassat et al., 2019). CV ranges were also contrasting between the groups; the INAcc-projection group displayed a range comparable to that of the main population, whereas the mNAccprojection group showed a much smaller range (INAcc CV median(IQR) $=48.95(35.47-$ 58.76) \%; mNAcc CV median(IQR) = 40.00(32.84-53.07) \%; Fig. 5.3B). One shared property that was observed in my data as well as in our lab's previous work was pause durations, which were shown to be higher in mNAcc-projecting DA neurons than in INAcc-projecting counterparts (Farassat et al., 2019). In addition, projection-defined in vivo DA neurons' responses to hyperpolarization also appeared to be different from the previous in vitro work; large sags and long rebound delays were present in both DA neuron subpopulations (Fig. 5.3D and 5.3E). Further recordings are needed to make any conclusions about the subthreshold membrane basis of DA neurons that drives firing activity and conveys behavior-relevant information in the two mesolimbic DA subpopulations when embedded in the network.

Figure 5.2. Combining in vivo patch-clamp method with retrograde tracing. To record from projection-defined dopamine neurons, retrograde tracer FG was infused into two major projection sites of dopamine neurons in the ventral striatum prior to recording. A) Schematics of infusion sites in nucleus accumbens lateral shell (INAcc) and medial shell (mNAcc). B) Experimental design. Left: Animal was anesthetized and head-fixed using a head-plate for recording. Top right: Recording pipette was lowered down to the midbrain. Bottom left: Recording was done in the whole-cell configuration which allowed NB (green dots) to fill the neuron. Red circles indicate vesicle-contained FG which had retrogradely traveled. C) Intrinsic blue fluorescence of FG in INAcc (left panel) and mNAcc (right panel). D) Anatomical distribution of projection-defined dopamine neurons recorded. E) Example recording trace from INAcc-projecting dopamine neuron with $\mathrm{V}_{\min }$ (blue) and $\mathrm{V}_{\text {thr }}$ (green) labeled. F) Immunolabeling of NB, TH and FG of the neuron in (D). G) Example recording from mNAcc-projecting dopamine neuron. H) Immunolabeling of the neuron in (G). FG: Fluoro-Gold; NG: neurobiotin; TH: tyrosine hydroxylase. Brain illustrations adapted with permission from G. Paxinos and K. Franklin, 2007. Copyright (2007) Elsevier. 
A

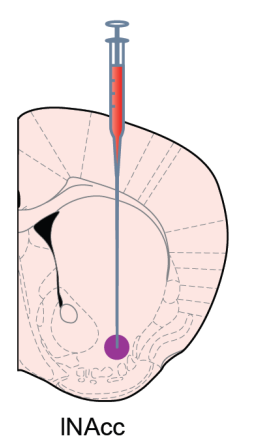

C
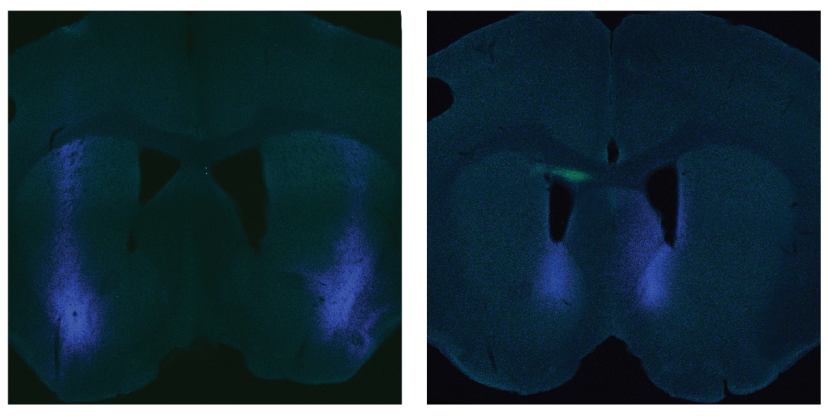

E

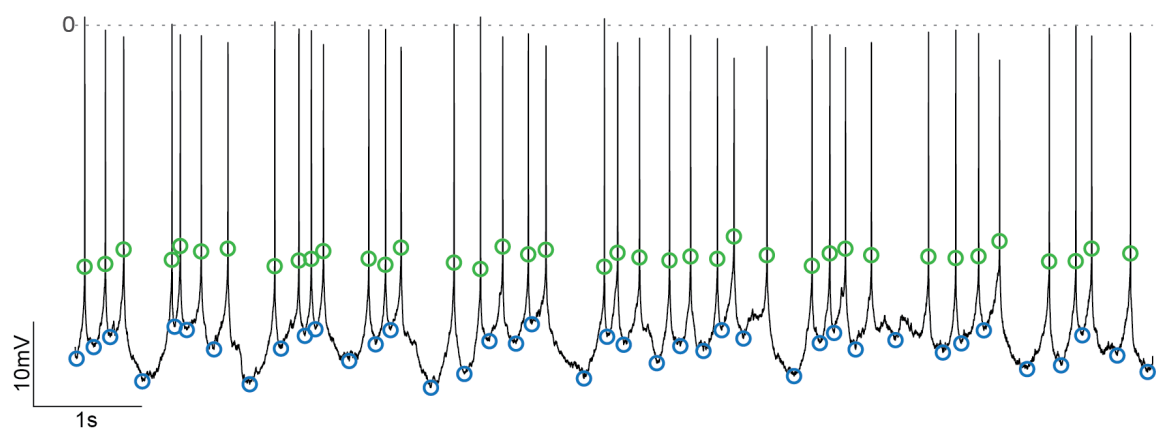

G

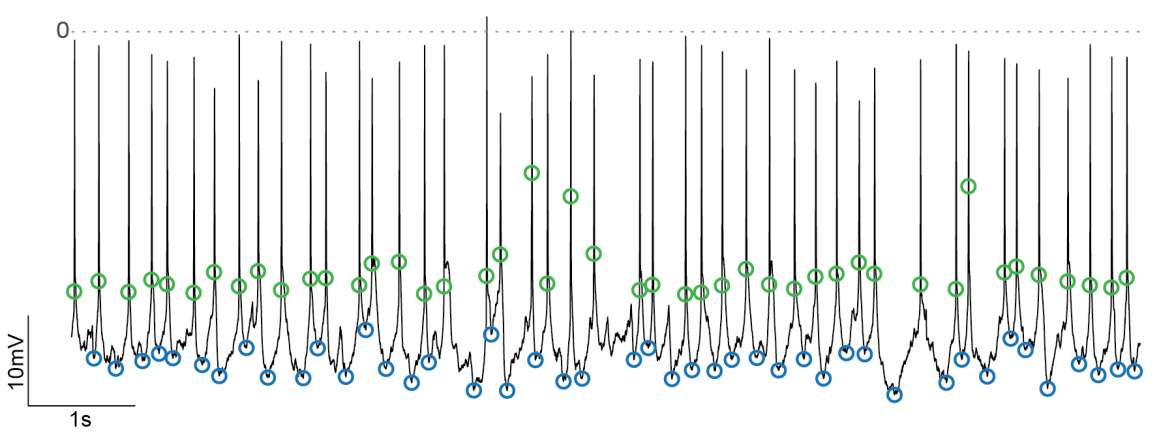

B
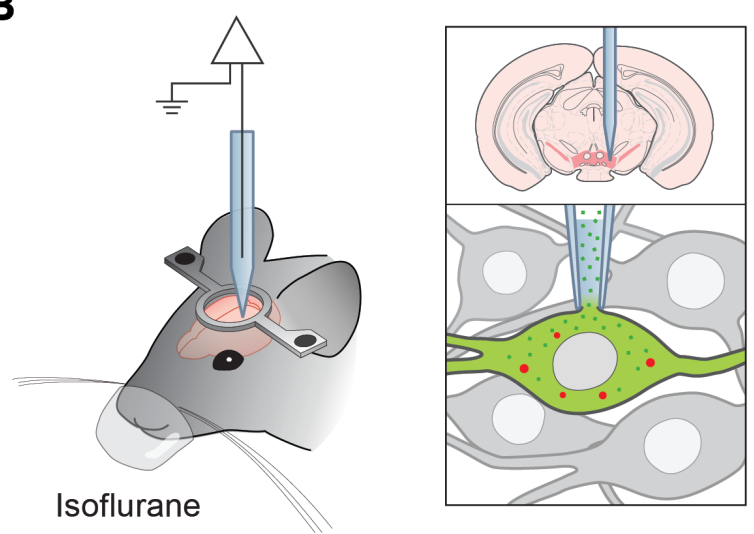

D

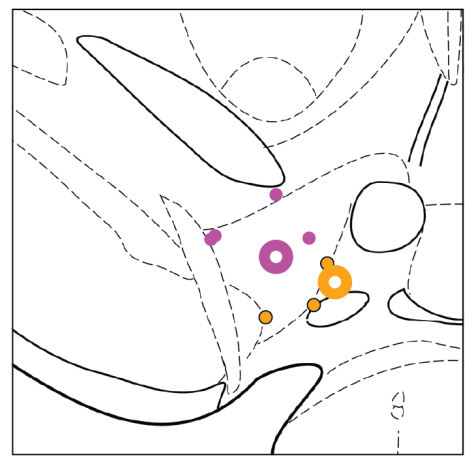

F

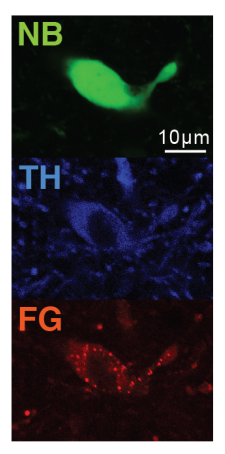

H

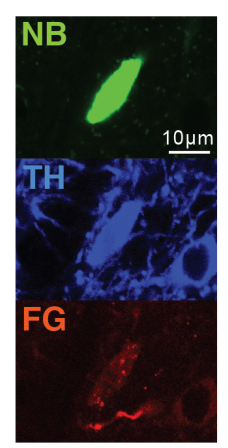


A

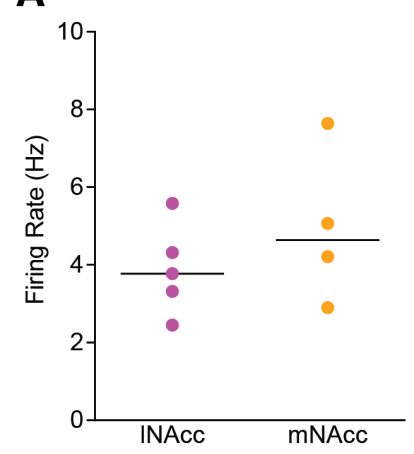

D

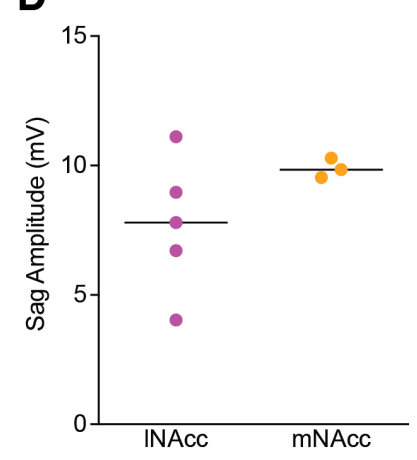

B

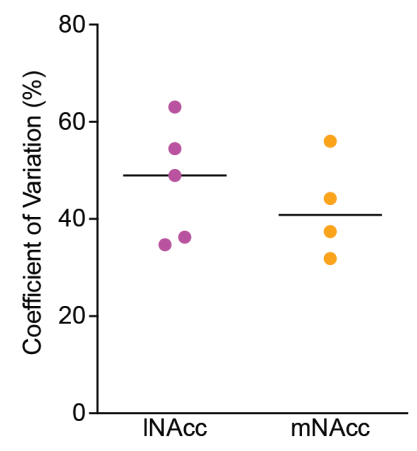

E

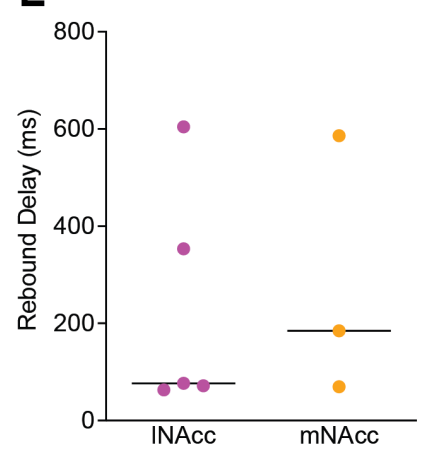

C

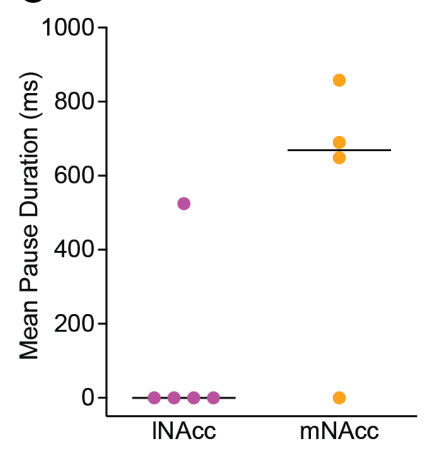

$\mathbf{F}$

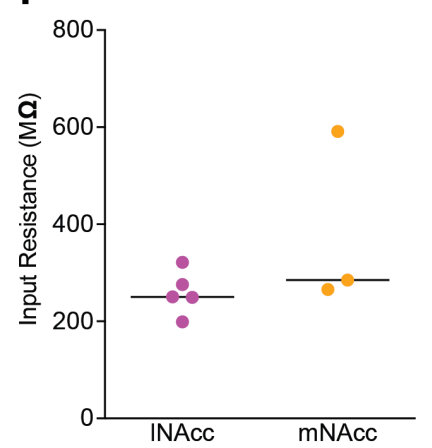

Figure 5.3. General electrophysiological properties of dopamine neurons projecting to nucleus accumbens lateral shell and medial shell. A) Mean firing rates of INAcc- and mNAcc-projecting DA neurons. B) Coefficient of variation. C) Mean pause duration was higher in dopamine neurons projecting to mNAcc. D) Sag amplitude. E) Rebound delay. F) Input resistance. INAcc: nucleus accumbens lateral shell; mNAcc: nucleus accumbens medial shell. 


\section{DISCUSSION}

Using the deep brain in vivo whole-cell patch-clamp method, I was able to gain new insights into the subthreshold membrane properties of in vivo midbrain DA neurons for the first time in over three decades. The electrophysiological activity of DA neurons recorded in the whole-cell mode was stable over time and was comparable to in vivo extracellular spontaneous activity of DA neurons as well as cell-attached firing activity recorded prior to breaking into the cell. Based on the systematic examination of my whole-cell recordings, I identified three distinct classes of subthreshold membrane behavior that were the basis of in vivo DA neuron firing patterns. The first class, observed in pacemaking and single-spiking (or tonic firing) DA neurons, was characterized by a unimodal distribution of subthreshold membrane potential minima $\left(\mathrm{V}_{\min }\right)$ as well as a unimodal distribution of action potential thresholds $\left(\mathrm{V}_{\mathrm{thr}}\right)$. The second class, which I named rebound bursting due to the pronounced hyperpolarization that precedes burst spike that signals the initiation of bursting, was associated with a bimodal distribution of $\mathrm{V}_{\min }$ and a unimodal distribution of $\mathrm{V}_{\text {thr. }}$. The third class was termed plateau bursting, in which burst spikes occurred and terminated at the top of transiently depolarized plateau potential. Plateau bursts were characterized by a bimodal distribution of $\mathrm{V}_{\text {thr }}$ and often a more complex multimodal distribution of $\mathrm{V}_{\min }$.

The general electrophysiological properties of identified midbrain DA neurons recorded by the method were in line with those obtained using sharp electrodes in the 1980s (Grace and Bunney, 1984a, 1984b, 1983a, 1983b). The only marked difference was found in the input resistance which was ten-fold higher in whole-cell recordings, likely due to the large leak introduced by the use of a sharp electrode (Li et al., 2004). In addition, analysis of subthreshold membrane activity in relation to ISI enabled for the first time identification of burst events according to subthreshold membrane behavior rather than relying solely on the ISI-based criteria originally proposed by Grace and Bunney (Grace and Bunney, 1984b). Importantly, some burst events detected by subthreshold membrane activity did not fulfill the traditional $80 \mathrm{~ms}-160 \mathrm{~ms}$ criteria. Subthreshold membrane dynamics thus allowed identification of phasic firing in a membrane activitydependent manner that may signal distinct information. 
$\mathrm{Ca}^{2+}$ is a particularly important molecule for neuronal processes and past studies have shown that changes in intracellular $\mathrm{Ca}^{2+}$ and $\mathrm{Ca}^{2+}$ buffer such as ethylene glycol-bis ( $\beta$-aminoethyl ether)- $\mathrm{N}, \mathrm{N}, \mathrm{N}^{\prime}, \mathrm{N}^{\prime}$-tetraacetic acid (EGTA) are known to distort DA neuronal firing (Catoni et al., 2019; Grace and Bunney, 1984b). The internal recording solution used for my project was adjusted to have a free $\mathrm{Ca}^{2+}$ concentration of approximately 80 $\mathrm{nM}$, a range closer to the physiological level of intracellular $\mathrm{Ca}^{2+}(\sim 100 \mathrm{nM})$ (Clapham, 2007). Comparisons with extracellular recordings confirmed that the use of this recording solution preserved both pacemaking as well as burst activity in in vivo DA neurons recorded in the whole-cell mode. However, while the majority of DA neurons in my dataset did not change their firing rates after breaking in, a few of them increased their firing rate by more than $1.5 \mathrm{~Hz}$ (Fig. 4.4C). This could be simply due to technical issues. For example, because the seal improves much more slowly in vivo, on-cell activity may have been recorded and terminated before it had stabilized. Another possibility would be the differential $\mathrm{Ca}^{2+}$ handling in distinct subpopulations of DA neurons as observed in the $\mathrm{Ca}^{2+}$-binding protein calbindin (CB)-positive and CB-negative DA neuron subpopulations which display different $\mathrm{Ca}^{2+}$ channel compositions (Evans et al., 2017). If the issue is indeed the internal recording solution, a perforated in vivo patch-clamp method would be useful, which forms pores in the membrane only permeable to monovalent cations and thus maintains endogenous $\mathrm{Ca}^{2+}$ and $\mathrm{Cl}^{-}$levels during recording (Hladky and Haydon, 1984; Linley, 2013).

Previous in vitro studies had shown that projection-defined DA neurons could be separated into two groups, conventional and atypical, based on their morphological and electrophysiological as well as molecular profiles (Lammel et al., 2008). In terms of electrophysiology, the conventional DA neurons were those projecting to the striatum and INAcc, which exhibited large sags and short rebound delays in response to negative current injections, while the atypical mesocorticolimbic DA neurons showed little to no sag and longer rebound delays to the same stimuli. In vivo DA neurons' responses to hyperpolarizing current injections did not possess such clearly-segregated phenotypes, and apart from one neuron with a rebound delay slope of longer than one second, no other in vivo DA neurons displayed the long rebound delay slopes observed in vitro, which ranged from 2.2 to 3.0 seconds (Lammel et al., 2008). Moreover, sag components of in 
vivo DA neurons were less prominent as compared to their in vitro counterparts, which was also reported previously (Grace and Onn,1989). Changes in conductances as a result of synaptic inputs might account for the large differences in the magnitude and variation of electrophysiological properties observed in in vivo versus in vitro DA neurons. Although the topographical organization of these properties, such as larger sag amplitudes in the medial SNc and lateral VTA and longer rebound delays in the medial VTA, was still in line with previous findings (Lammel et al., 2008), this categorization does not fully apply to and is incomplete for describing the results of in vivo DA neurons. One novel finding was the two neurons in the lateral SNc which exhibited rebound delays longer than one second, but with what appeared to be failed spikes instead of a depolarizing linear slope, suggesting that mechanisms other than A-type $\mathrm{K}^{+}$channels are responsible for the long rebound delays in these neurons.

Of the 67 DA neurons analyzed in my study, 41 neurons were regular or irregular tonic spikers, 20 neurons were rebound bursters, two were plateau bursters, and four had both rebound and plateau burst patterns. Tonic firing of in vivo DA neurons proved to be highly stable and maintained the level of $V_{\min }$ and $V_{\text {thr }}$ despite the network influence. Considering the resemblance to the activity of synaptically-isolated in vitro DA neurons, these in vivo DA neurons are relatively autonomous and their spiking is mainly controlled by intrinsic mechanisms while still allowing some degree of extrinsic influence as evidenced by irregular spiking and occasional bursts. In comparison, GABAergic control may be key to rebound bursts, based on the nature of subthreshold membrane potential behavior. Considering that the majority of synaptic inputs onto the SNc are GABAergic (Bolam and Smith, 1990) and the general strong inhibitory influence midbrain DA neurons receive from the striatum, GP, SNr, as well as the RMTg, rhythmic rebound bursts may be controlled by these GABAergic inputs, most of which are part of the basal ganglia feedback loops. Blocking $\mathrm{GABA}_{\mathrm{A}}$ receptors in the SNc is indeed known to switch DA neurons into the burst firing mode (Tepper and Lee, 2007). A computational study showed that a GABAergic tone combined with the activation of K-ATP channels in the presence of NMDA induces rhythmic burst firing in mSNc DA neurons, which resembled in vivo rebound bursting (Knowlton et al., 2018). Another candidate mechanism for DA rebound bursting is suppression of the small-conductance $\mathrm{Ca}^{2+}$-activated $\mathrm{K}^{+}(\mathrm{SK})$ channel 
by transient activation of $\mathrm{GABA}_{\mathrm{B}}$ receptor, as it increases firing rate and irregularity (Estep et al., 2016). It is indeed likely that distinct DA neuron subpopulations employ different biophysical mechanisms for rebound bursts.

Despite the heterogeneity, DA neurons show a great level of topographical organization within the midbrain according to projection and function (Farassat et al., 2019; Haber, 2014; Ikemoto, 2007; Lammel et al., 2014, 2008). However, due to the broad distribution of rebound bursters in the anatomical map (Fig. 4.17C), it was difficult to pinpoint to their potential functional roles at this time. Rhythmic burst patterns or oscillations had been observed in DA neurons during a wide range of states including during a working memory task (Duvarci et al., 2018; Fujisawa and Buzsáki, 2011), prior to food consumption and during rapid eye movement (REM) sleep (Dahan et al., 2007) (also see Section 5.1). The frequency of cumulative rebound burst $V_{\min }$ was in the lower delta range (mean $\pm \mathrm{SD}=1.10 \pm 0.49$ ) while that reported during working memory tasks was in the higher delta range ( $\sim 4 \mathrm{~Hz}$ ) (Duvarci et al., 2018; Fujisawa and Buzsáki, 2011). Alternatively, the rhythmic activity is possibly related to the use of isoflurane, as the choice of anesthesia determines the brain state which in turn affects bursting (Hyland et al., 2002; Marinelli and McCutcheon, 2014; Overton and Clark, 1997; Steinfels et al., 1981). In thalamocortical neurons, rhythmic burst patterns are observed during slow-wave sleep while single-spiking patterns are more common during wakefulness (Llinás and Steriade, 2006).

In contrast to rebound bursts, plateau bursts may be driven by a shift in excitatory-inhibitory (E/I) balance. Only two DA neurons in my dataset showed solely this type of bursting, which could be due to the reduced excitatory inputs under anesthetized conditions, or due to the small population of DA neurons that displays plateau bursting. Interestingly, both of these neurons were found in the medial VTA and displayed rebound delays longer than $500 \mathrm{~ms}$, which fits the description of "atypical" DA subpopulations in the medial VTA described by Lammel et al., which are part of the mesocorticolimbic pathways (Lammel et al., 2008). Moreover, long rebound delays observed in these neurons suggest that their distinct $I_{A}$ kinetics may be part of the intrinsic mechanism that shapes plateau bursting (Tarfa et al., 2017). The consistent presence of large sags in rebound bursters and long rebound delays in plateau bursters 
is a strong indication that intrinsic properties likely determine DA neurons' burst capacity. Supporting this idea, during positive current injections, plateau bursts were observed only in plateau bursting and rebound-plateau bursting DA neurons, whereas tonic and rebound bursting neurons never exhibited plateaus and failed to reach intraburst frequencies of $100 \mathrm{~Hz}$ that plateau bursts consistently showed (not shown).

The present study may also explain the paradoxical observations of ISIs in bursts described in literature. Grace and Bunney originally described bursts as having increasing ISIs (Grace and Bunney, 1984b), whereas Hyland and colleagues reported that ISIs shortened during bursts in freely-moving rats (Hyland et al., 2002). The burst events observed in the former paper are similar to rebound bursts in this study, in which most ISIs increased in duration toward burst termination (Fig. 4.9A), although some bursts that initiated earlier in the disinhibition wave had a slightly longer first ISI of burst as compared to the following ISI. On the other hand, bursts described for freely-moving animals are more in line with plateau bursts, which always had the shortest ISI at the end of bursts (Fig. 4.10A). As plateau bursts provide a temporally precise DA signaling as opposed to the less precisely controlled rebound bursts, time-sensitive reward and aversive events and their cues may evoke plateau bursting in DA neurons. Importantly, the two burst types covered different frequency ranges: most rebound bursts were in the $10-30 \mathrm{~Hz}$ range, while plateau bursts were in the range of $50 \mathrm{up}$ to $170 \mathrm{~Hz}$. Whole-cell recordings of DA neurons in awake animals will be necessary to determine whether rhythmic rebound bursting and plateau bursting described in this study are indeed exhibited by DA neurons in behaving animals and to understand their relevance to behavior. Moreover, the existence of distinct types of phasic firing should be taken into consideration when interpreting results from behavioral experiments, as they may encode different information.

A striking electrophysiological property of the in vivo DA neuron population was that their subthreshold membrane activity during ISI directly affected the duration of ISI. Even the most regular pacemaker neurons exhibited a negative correlation between ISI and corresponding membrane potential minima $\left(\mathrm{V}_{\min }\right)$, suggesting that DA neurons are constantly integrating synaptic and neuromodulatory influences into their intrinsic spike activity. This property was not displayed by neighboring non-DA neurons, on the other 
hand. There were also a small number of DA neurons in which $V_{\min }$ and ISI did not correlate, and these cells had shared electrophysiological characteristics. First, their ISIs were noisy and rapidly fluctuating, and second, they had long rebound delays in response to termination of hyperpolarization (range $=353.35$ to $1207.9 \mathrm{~ms}$ ). Long rebound delays are mediated by A-type $\mathrm{K}^{+}$channels expressed in SNc and VTA DA neurons which enhance the duration of GABAergic inhibition (Khaliq and Bean, 2008; Tarfa et al., 2017). This prolongation of inhibition depends on the strength and duration of GABAergic inputs, but the duration has a ceiling effect and is restricted to certain lengths, which could partly explain the non-correlative relationship between ISI and $V_{\min }$ in these DA neurons (Tarfa et al., 2017). Interestingly, not all pauses showed such highly fluctuating subthreshold membrane behavior, although it was common for longer pauses. Other pauses consisted of multiple spike failures and depolarization blocks in which membrane potentials stayed at depolarized (or plateau-like) membrane voltages, likely due to inactivation of voltagegated $\mathrm{Na}^{+}$channels (Tucker et al., 2012) which only recovered after hyperpolarization. While the relationship between $V_{\text {min }}$ and firing pattern was examined, subthreshold behavior of pauses was left out in this study. As pauses in DA neurons constitute a crucial part of reward omission signaling, aversion and related cues, further study is necessary to understand the mechanisms of different types of pauses and what information they convey in their circuits.

Similar to $V_{\text {min }}, V_{\text {thr }}$ of DA neurons also showed correlations with their corresponding ISIs. However, in contrast to $V_{\min }$ which appeared to drive the ISI, spike thresholds seemed to adapt to the preceding membrane potential activity. Recent in vivo and modeling studies have indeed indicated that action potential thresholds adapt to the recent membrane potential history at a short timescale, which enables robust computation of input signals (Fontaine et al., 2014; Huang et al., 2016). At least in other systems, inactivation of voltage-gated $\mathrm{Na}^{+}$channels and $\mathrm{K}^{+}$channel activation had been shown to underlie the dynamic changes of in vivo spike thresholds (Higgs and Spain, 2011; Platkiewicz and Brette, 2011). In my dataset, around and during bursts when threshold adaptation was particularly noticeable, spike waveform broadened and rate of depolarization as well as spike amplitude decreased toward the end of burst and 
recovered prior to subsequent bursts, which points to the involvement of voltage-gated $\mathrm{Na}^{+}$channels.

In classifying DA neurons by subthreshold behavior-based firing patterns for population analysis, identification of $\mathrm{V}_{\min }$ and $\mathrm{V}_{\text {thr }}$ distributions proved challenging using conventional bimodality detection methods, particularly for neurons that had relatively fewer phasic events. Kernel density derivative estimation on the other hand accurately detected modes in $V_{\min }$ and $V_{\text {thr }}$ distributions, but care should be taken to choose the right bandwidth for each dataset under investigation as it highly affects the outcome of mode detection. Alternatively, $\mathrm{BC}$ of $\mathrm{V}_{\min }$ and $\mathrm{BC}$ of $\mathrm{V}_{\text {thr }}$, which are easily calculated based on the sample size, skewness and kurtosis of the distribution (Pfister et al., 2013), can be used to describe the subthreshold-based firing characteristics of in vivo midbrain DA neurons in a continuous 2D space without defining a threshold of bimodality detection. Unfortunately, the sample size of plateau burst and rebound-plateau bursting neurons was small in my dataset, rendering the two quadrants in Figure 4.16B partially empty. Greater sample size of these neurons is needed to confirm the validity of this distribution plot.

While the use of in vivo patch-clamp method provides essential information on subthreshold membrane properties during burst firing, it is not always the method of choice, particularly for freely-moving behavioral studies. Dissection of intrinsic mechanisms of phasic firing during such behaviors may thus be difficult at this time. However, CV proved surprisingly indicative of the subthreshold basis of tonic and phasic firing patterns of in vivo DA neurons, for the most part (Fig. 4.15A). Since CV is an indicator of ISI variabilities and ISI in turn correlates with both $\mathrm{V}_{\min }$ and $\mathrm{V}_{\text {thr, }} \mathrm{CV}$ is ultimately a parameter that reflects both $\mathrm{V}_{\min }$ and $\mathrm{V}_{\text {thr }}$ properties of in vivo DA neuron activity. Importantly, CV is a value that can be obtained with extracellular recording, which means that one can make predictions about the subthreshold membrane basis of extracellularly-recorded DA neuron spiking by referring to the data obtained from in vivo patch-clamp, such as the plot shown in Figure 4.15A. In addition, plateau bursts tend to result in instantaneous firing rates greater than $50 \mathrm{~Hz}$, which can also help identify the subthreshold membrane activity of in vivo DA neurons. 
Nevertheless, a novel approach was needed to improve our understanding of phasic firing in DA neurons, and this study demonstrates that the deep brain in vivo patchclamp technique is a valuable tool for this purpose. This method can be subsequently combined with pharmacological manipulations and/or dynamic clamp to further identify the intrinsic and extrinsic basis of rebound and plateau bursting described in this study. Furthermore, future studies in awake behaving animals will be crucial for dissecting the biophysical mechanisms of DA neuron's tonic and phasic firing that encode reward-, aversion- and cue-related behaviors, as well as locomotion, salience, and cognition. The deep brain in vivo patch-clamp method thus adds to the existing tools for dissecting the DA system and will help address some open questions in the field, particularly regarding the mechanisms underlying phasic firing, which is an essential component of midbrain DA neurons controlling behavior. 


\section{CONCLUSIONS}

In order to expand our current knowledge of biophysical properties of DA neuron's phasic firing, I have established the deep-brain whole-cell patch-clamp method to record the subthreshold membrane activity of midbrain DA neurons in the intact brain. My first systematic analysis of DA neuron's subthreshold membrane behavior revealed the close relationship between firing activity and patterns of DA neurons and the behavior of subthreshold membrane potentials, and that tonic and phasic firing patterns of in vivo midbrain DA neurons can be classified based on three distinct subthreshold membrane signatures: 1) tonic firing, which is characterized by stable, non-fluctuating subthreshold membrane potentials; 2) rebound bursting, which is characterized by prominent hyperpolarizations that initiate bursting; and 3) plateau bursting, which is characterized by transient, depolarized plateaus on which bursting terminates. The findings highlighted the existence of different mechanisms of phasic firing, which may encode distinct types of information. Taken together, this study provides the first indepth evaluation of subthreshold membrane dynamics that drive distinct tonic and phasic firing patterns in DA neurons in vivo, and this method can be used to further investigate the detailed intrinsic biophysical mechanisms of phasic activity in the intact brain that provides the basis of everyday behavior. 


\section{REFERENCES}

Albin, R.L., 1995. The pathophysiology of chorea/ballism and Parkinsonism. Parkinsonism Relat. Disord. 1, 3-11. doi:10.1016/1353-8020(95)00011-t

Albin, R.L., Young, A.B., Penney, J.B., 1989. The functional anatomy of basal ganglia disorders. Trends Neurosci. 12, 366-375. doi:10.1016/0166-2236(89)90074-x

Albrecht, U., 2020. Molecular Connections Between Circadian Clocks and Mood-related Behaviors. J. Mol. Biol. 432, 3714-3721. doi:10.1016/j.jmb.2019.11.021

Alexander, S.P.H., Mathie, A., Peters, J.A., 2011. ION CHANNELS. Br. J. Pharmacol. 164, S137-S174. doi:10.1111/j.1476-5381.2011.01649_5.x

Andén, N.E., Hfuxe, K., Hamberger, B., Hökfelt, T., 1966. A quantitative study on the nigro-neostriatal dopamine neuron system in the rat. Acta Physiol Scand 67, 306-312. doi:10.1111/j.17481716.1966.tb03317.x

Arora, D., Hearing, M., Haluk, D.M., Mirkovic, K., Fajardo-Serrano, A., Wessendorf, M.W., Watanabe, M., Luján, R., Wickman, K., 2011. Acute cocaine exposure weakens GABA(B) receptor-dependent Gprotein-gated inwardly rectifying $\mathrm{K}+$ signaling in dopamine neurons of the ventral tegmental area. J. Neurosci. 31, 12251-12257. doi:10.1523/JNEUROSCI.0494-11.2011

Ashton, A., Jagannath, A., 2020. Disrupted sleep and circadian rhythms in schizophrenia and their interaction with dopamine signaling. Front. Neurosci. 14, 636. doi:10.3389/fnins.2020.00636

Azevedo, F.A.C., Carvalho, L.R.B., Grinberg, L.T., Farfel, J.M., Ferretti, R.E.L., Leite, R.E.P., Jacob Filho, W., Lent, R., Herculano-Houzel, S., 2009. Equal numbers of neuronal and nonneuronal cells make the human brain an isometrically scaled-up primate brain. J. Comp. Neurol. 513, 532-541. doi:10.1002/cne.21974

Barger, G., Dale, H.H., 1910. Chemical structure and sympathomimetic action of amines. J. Physiol. (Lond.) 41, 19-59. doi:10.1113/jphysiol.1910.sp001392

Beaulieu, J.-M., Gainetdinov, R.R., 2011. The physiology, signaling, and pharmacology of dopamine receptors. Pharmacol. Rev. 63, 182-217. doi:10.1124/pr.110.002642

Beier, K.T., Steinberg, E.E., DeLoach, K.E., Xie, S., Miyamichi, K., Schwarz, L., Gao, X.J., Kremer, E.J., Malenka, R.C., Luo, L., 2015. Circuit Architecture of VTA Dopamine Neurons Revealed by Systematic Input-Output Mapping. Cell 162, 622-634. doi:10.1016/j.cell.2015.07.015

Belin, D., Everitt, B.J., 2008. Cocaine seeking habits depend upon dopamine-dependent serial connectivity linking the ventral with the dorsal striatum. Neuron 57, 432-441. doi:10.1016/j.neuron.2007.12.019

Benarroch, E.E., 2010. Neuronal voltage-gated calcium channels: brief overview of their function and clinical implications in neurology. Neurology 74, 1310-1315. doi:10.1212/WNL.0b013e3181da364b

Benes, F.M., 2001. Carlsson and the discovery of dopamine. Trends Pharmacol. Sci. 22, 46-47. doi:10.1016/S0165-6147(00)01607-2

Benkert, J., Hess, S., Roy, S., Beccano-Kelly, D., Wiederspohn, N., Duda, J., Simons, C., Patil, K., Gaifullina, A., Mannal, N., Dragicevic, E., Spaich, D., Müller, S., Nemeth, J., Hollmann, H., Deuter, N., Mousba, Y., Kubisch, C., Poetschke, C., Striessnig, J., Pongs, O., Schneider, T., Wade-Martins, R., Patel, S., Parlato, R., Frank, T., Kloppenburg, P., Liss, B., 2019. Cav2.3 channels contribute to dopaminergic neuron loss in a model of Parkinson's disease. Nat. Commun. 10, 5094. doi:10.1038/s41467-019-12834-x

Berridge, K.C., 2007. The debate over dopamine's role in reward: the case for incentive salience. Psychopharmacology 191, 391-431. doi:10.1007/s00213-006-0578-x

Bers, D.M., Patton, C.W., Nuccitelli, R., 2010. A practical guide to the preparation of $\mathrm{Ca}(2+)$ buffers. Methods Cell Biol. 99, 1-26. doi:10.1016/B978-0-12-374841-6.00001-3

Björklund, A., Dunnett, S.B., 2007. Dopamine neuron systems in the brain: an update. Trends Neurosci. 30, 194-202. doi:10.1016/j.tins.2007.03.006

Bolam, J.P., Hanley, J.J., Booth, P.A., Bevan, M.D., 2000. Synaptic organisation of the basal ganglia. J. Anat. 196 ( Pt 4), 527-542. doi:10.1046/j.1469-7580.2000.19640527.x

Bolam, J.P., Pissadaki, E.K., 2012. Living on the edge with too many mouths to feed: why dopamine 
neurons die. Mov. Disord. 27, 1478-1483. doi:10.1002/mds.25135

Bolam, J.P., Smith, Y., 1990. The GABA and substance $P$ input to dopaminergic neurones in the substantia nigra of the rat. Brain Res. 529, 57-78. doi:10.1016/0006-8993(90)90811-o

Borroto-Escuela, D.O., Perez De La Mora, M., Manger, P., Narváez, M., Beggiato, S., Crespo-Ramírez, M., Navarro, G., Wydra, K., Díaz-Cabiale, Z., Rivera, A., Ferraro, L., Tanganelli, S., Filip, M., Franco, R., Fuxe, K., 2018. Brain dopamine transmission in health and parkinson's disease: modulation of synaptic transmission and plasticity through volume transmission and dopamine heteroreceptors. Front. Synaptic Neurosci. 10, 20. doi:10.3389/fnsyn.2018.00020

Botev, Z., 2015. Kernel Density Estimator [WWW Document]. MATLAB Central File Exchange. URL https://www.mathworks.com/matlabcentral/fileexchange/14034-kernel-density-estimator (accessed 2.20.21).

Botev, Z.I., Grotowski, J.F., Kroese, D.P., 2010. Kernel density estimation via diffusion. Ann. Statist. 38, 2916-2957. doi:10.1214/10-AOS799

Bouarab, C., Thompson, B., Polter, A.M., 2019. VTA GABA neurons at the interface of stress and reward. Front. Neural Circuits 13, 78. doi:10.3389/fncir.2019.00078

Bouyer, J.J., Joh, T.H., Pickel, V.M., 1984. Ultrastructural localization of tyrosine hydroxylase in rat nucleus accumbens. J. Comp. Neurol. 227, 92-103. doi:10.1002/cne.902270110

Braak, H., Del Tredici, K., 2004. Poor and protracted myelination as a contributory factor to neurodegenerative disorders. Neurobiol. Aging 25, 19-23. doi:10.1016/j.neurobiolaging.2003.04.001

Brazhnik, E., Shah, F., Tepper, J.M., 2008. GABAergic afferents activate both GABAA and GABAB receptors in mouse substantia nigra dopaminergic neurons in vivo. J. Neurosci. 28, 10386-10398. doi:10.1523/JNEUROSCI.2387-08.2008

Brischoux, F., Chakraborty, S., Brierley, D.I., Ungless, M.A., 2009. Phasic excitation of dopamine neurons in ventral VTA by noxious stimuli. Proc. Natl. Acad. Sci. USA 106, 4894-4899. doi:10.1073/pnas.0811507106

Bromberg-Martin, E.S., Matsumoto, M., Hikosaka, O., 2010. Dopamine in motivational control: rewarding, aversive, and alerting. Neuron 68, 815-834. doi:10.1016/j.neuron.2010.11.022

Brown, D.A., Passmore, G.M., 2009. Neural KCNQ (Kv7) channels. Br. J. Pharmacol. 156, 1185-1195. doi:10.1111/j.1476-5381.2009.00111.x

Cain, S.M., Snutch, T.P., 2010. Contributions of T-type calcium channel isoforms to neuronal firing. Channels 4, 475-482. doi:10.4161/chan.4.6.14106

Calabresi, P., Picconi, B., Tozzi, A., Ghiglieri, V., Di Filippo, M., 2014. Direct and indirect pathways of basal ganglia: a critical reappraisal. Nat. Neurosci. 17, 1022-1030. doi:10.1038/nn.3743

Cardozo, D.L., Bean, B.P., 1995. Voltage-dependent calcium channels in rat midbrain dopamine neurons: modulation by dopamine and GABAB receptors. J. Neurophysiol. 74, 1137-1148. doi:10.1152/jn.1995.74.3.1137

Carlsson, A., Lindqvist, M., Magnusson, T., 1957. 3,4-Dihydroxyphenylalanine and 5-hydroxytryptophan as reserpine antagonists. Nature 180, 1200. doi:10.1038/1801200a0

Carlsson, A., Lindqvist, M., Magnusson, T., Waldeck, B., 1958. On the presence of 3-hydroxytyramine in brain. Science 127, 471. doi:10.1126/science.127.3296.471

Cartier, E.A., Parra, L.A., Baust, T.B., Quiroz, M., Salazar, G., Faundez, V., Egaña, L., Torres, G.E., 2010. A biochemical and functional protein complex involving dopamine synthesis and transport into synaptic vesicles. J. Biol. Chem. 285, 1957-1966. doi:10.1074/jbc.M109.054510

Catoni, C., Calì, T., Brini, M., 2019. Calcium, dopamine and neuronal calcium sensor 1: their contribution to parkinson's disease. Front. Mol. Neurosci. 12, 55. doi:10.3389/fnmol.2019.00055

Cazorla, M., de Carvalho, F.D., Chohan, M.O., Shegda, M., Chuhma, N., Rayport, S., Ahmari, S.E., Moore, H., Kellendonk, C., 2014. Dopamine D2 receptors regulate the anatomical and functional balance of basal ganglia circuitry. Neuron 81, 153-164. doi:10.1016/j.neuron.2013.10.041

Cazorla, M., Kang, U.J., Kellendonk, C., 2015. Balancing the basal ganglia circuitry: a possible new role for dopamine D2 receptors in health and disease. Mov. Disord. 30, 895-903. doi:10.1002/mds.26282

Chacón, J.E., Duong, T., 2020. Multivariate Kernel Smoothing and Its Applications, 1st ed. Chapman and Hall/CRC.

Chan, C.S., Guzman, J.N., Ilijic, E., Mercer, J.N., Rick, C., Tkatch, T., Meredith, G.E., Surmeier, D.J., 2007. 
"Rejuvenation" protects neurons in mouse models of Parkinson's disease. Nature 447, 10811086. doi:10.1038/nature05865

Chang, C.Y., Esber, G.R., Marrero-Garcia, Y., Yau, H.-J., Bonci, A., Schoenbaum, G., 2016. Brief optogenetic inhibition of dopamine neurons mimics endogenous negative reward prediction errors. Nat. Neurosci. 19, 111-116. doi:10.1038/nn.4191

Chen, B.T., Patel, J.C., Moran, K.A., Rice, M.E., 2011. Differential calcium dependence of axonal versus somatodendritic dopamine release, with characteristics of both in the ventral tegmental area. Front. Syst. Neurosci. 5, 39. doi:10.3389/fnsys.2011.00039

Chevalier, G., Deniau, J.M., 1990. Disinhibition as a basic process in the expression of striatal functions. Trends Neurosci. 13, 277-280. doi:10.1016/0166-2236(90)90109-n

Chiodo, L.A., Bannon, M.J., Grace, A.A., Roth, R.H., Bunney, B.S., 1984. Evidence for the absence of impulse-regulating somatodendritic and synthesis-modulating nerve terminal autoreceptors on subpopulations of mesocortical dopamine neurons. Neuroscience 12, 1-16. doi:10.1016/03064522(84)90133-7

Chuhma, N., Mingote, S., Kalmbach, A., Yetnikoff, L., Rayport, S., 2017. Heterogeneity in dopamine neuron synaptic actions across the striatum and its relevance for schizophrenia. Biol. Psychiatry 81, 43-51. doi:10.1016/j.biopsych.2016.07.002

Chung, S., Lee, E.J., Yun, S., Choe, H.K., Park, S.-B., Son, H.J., Kim, K.-S., Dluzen, D.E., Lee, I., Hwang, O., Son, G.H., Kim, K., 2014. Impact of circadian nuclear receptor REV-ERB $\alpha$ on midbrain dopamine production and mood regulation. Cell 157, 858-868. doi:10.1016/j.cell.2014.03.039

Clapham, D.E., 2007. Calcium signaling. Cell 131, 1047-1058. doi:10.1016/j.cell.2007.11.028

Creed, M.C., Ntamati, N.R., Tan, K.R., 2014. VTA GABA neurons modulate specific learning behaviors through the control of dopamine and cholinergic systems. Front. Behav. Neurosci. 8, 8. doi:10.3389/fnbeh.2014.00008

Crow, T.J., 1972. Catecholamine-containing neurones and electrical self-stimulation. 1. A review of some data. Psychol. Med. 2, 414-421. doi:10.1017/s0033291700045232

Cruz, H.G., Ivanova, T., Lunn, M.-L., Stoffel, M., Slesinger, P.A., Lüscher, C., 2004. Bi-directional effects of GABA(B) receptor agonists on the mesolimbic dopamine system. Nat. Neurosci. 7, 153-159. doi:10.1038/nn1181

Cui, G., Jun, S.B., Jin, X., Pham, M.D., Vogel, S.S., Lovinger, D.M., Costa, R.M., 2013. Concurrent activation of striatal direct and indirect pathways during action initiation. Nature 494, 238-242. doi:10.1038/nature11846

Cumming, P., 2011. Absolute abundances and affinity states of dopamine receptors in mammalian brain: A review. Synapse 65, 892-909. doi:10.1002/syn.20916

da Silva, J.A., Tecuapetla, F., Paixão, V., Costa, R.M., 2018. Dopamine neuron activity before action initiation gates and invigorates future movements. Nature 554, 244-248. doi:10.1038/nature25457

Dahan, L., Astier, B., Vautrelle, N., Urbain, N., Kocsis, B., Chouvet, G., 2007. Prominent burst firing of dopaminergic neurons in the ventral tegmental area during paradoxical sleep. Neuropsychopharmacology 32, 1232-1241. doi:10.1038/sj.npp.1301251

Dahlstroem, A., Fuxe, K., 1964. Evidence for the existence of monoamine-containing neurons in the central nervous system. i. demonstration of monoamines in the cell bodies of brain stem neurons. Acta Physiol Scand Suppl SUPPL 232:1-55.

Dal Bo, G., St-Gelais, F., Danik, M., Williams, S., Cotton, M., Trudeau, L.-E., 2004. Dopamine neurons in culture express VGLUT2 explaining their capacity to release glutamate at synapses in addition to dopamine. J. Neurochem. 88, 1398-1405. doi:10.1046/j.1471-4159.2003.02277.x

Davie, C.A., 2008. A review of Parkinson's disease. Br Med Bull 86, 109-127. doi:10.1093/bmb/ldn013

De Deurwaerdère, P., Di Giovanni, G., 2017. Serotonergic modulation of the activity of mesencephalic dopaminergic systems: Therapeutic implications. Prog. Neurobiol. 151, 175-236. doi:10.1016/j.pneurobio.2016.03.004

de Jong, J.W., Afjei, S.A., Pollak Dorocic, I., Peck, J.R., Liu, C., Kim, C.K., Tian, L., Deisseroth, K., Lammel, S., 2019. A neural circuit mechanism for encoding aversive stimuli in the mesolimbic dopamine system. Neuron 101, 133-151.e7. doi:10.1016/j.neuron.2018.11.005

DeLong, M.R., 1990. Primate models of movement disorders of basal ganglia origin. Trends Neurosci. 13, 
281-285. doi:10.1016/0166-2236(90)90110-v

Deschênes, M., Paradis, M., Roy, J.P., Steriade, M., 1984. Electrophysiology of neurons of lateral thalamic nuclei in cat: resting properties and burst discharges. J. Neurophysiol. 51, 1196-1219. doi:10.1152/jn.1984.51.6.1196

Dreyer, J.K., Herrik, K.F., Berg, R.W., Hounsgaard, J.D., 2010. Influence of phasic and tonic dopamine release on receptor activation. J. Neurosci. 30, 14273-14283. doi:10.1523/JNEUROSCI.189410.2010

Durante, P., Cardenas, C.G., Whittaker, J.A., Kitai, S.T., Scroggs, R.S., 2004. Low-threshold L-type calcium channels in rat dopamine neurons. J. Neurophysiol. 91, 1450-1454. doi:10.1152/jn.01015.2003

Duvarci, S., Simpson, E.H., Schneider, G., Kandel, E.R., Roeper, J., Sigurdsson, T., 2018. Impaired recruitment of dopamine neurons during working memory in mice with striatal D2 receptor overexpression. Nat. Commun. 9, 2822. doi:10.1038/s41467-018-05214-4

Eiden, L.E., Schäfer, M.K.-H., Weihe, E., Schütz, B., 2004. The vesicular amine transporter family (SLC18): amine/proton antiporters required for vesicular accumulation and regulated exocytotic secretion of monoamines and acetylcholine. Pflugers Arch. 447, 636-640. doi:10.1007/s00424-003-1100-5

Enna, S.J., 2007. The GABA Receptors, in: Enna, S.J., Möhler, H. (Eds.), The GABA Receptors. Humana Press, Totowa, NJ, pp. 1-21. doi:10.1007/978-1-59745-465-0_1

Eshel, N., Bukwich, M., Rao, V., Hemmelder, V., Tian, J., Uchida, N., 2015. Arithmetic and local circuitry underlying dopamine prediction errors. Nature 525, 243-246. doi:10.1038/nature14855

Estep, C.M., Galtieri, D.J., Zampese, E., Goldberg, J.A., Brichta, L., Greengard, P., Surmeier, D.J., 2016. Transient activation of GABAB receptors suppresses $S K$ channel currents in substantia nigra pars compacta dopaminergic neurons. PLoS One 11, e0169044. doi:10.1371/journal.pone.0169044

Evans, R.C., Zhu, M., Khaliq, Z.M., 2017. Dopamine Inhibition Differentially Controls Excitability of Substantia Nigra Dopamine Neuron Subpopulations through T-Type Calcium Channels. J. Neurosci. 37, 3704-3720. doi:10.1523/JNEUROSCI.0117-17.2017

Everitt, B.J., Robbins, T.W., 2005. Neural systems of reinforcement for drug addiction: from actions to habits to compulsion. Nat. Neurosci. 8, 1481-1489. doi:10.1038/nn1579

Farassat, N., Costa, K.M., Stojanovic, S., Albert, S., Kovacheva, L., Shin, J., Egger, R., Somayaji, M., Duvarci, S., Schneider, G., Roeper, J., 2019. In vivo functional diversity of midbrain dopamine neurons within identified axonal projections. Elife 8. doi:10.7554/eLife.48408

Ferguson, S.M., 2018. Axonal transport and maturation of lysosomes. Curr. Opin. Neurobiol. 51, 45-51. doi:10.1016/j.conb.2018.02.020

Floresco, S.B., West, A.R., Ash, B., Moore, H., Grace, A.A., 2003. Afferent modulation of dopamine neuron firing differentially regulates tonic and phasic dopamine transmission. Nat. Neurosci. 6, 968-973. doi:10.1038/nn1103

Fontaine, B., Peña, J.L., Brette, R., 2014. Spike-threshold adaptation predicted by membrane potential dynamics in vivo. PLoS Comput. Biol. 10, e1003560. doi:10.1371/journal.pcbi.1003560

Ford, C.P., 2014. The role of D2-autoreceptors in regulating dopamine neuron activity and transmission. Neuroscience 282, 13-22. doi:10.1016/j.neuroscience.2014.01.025

Francis, T.C., Lobo, M.K., 2017. Emerging role for nucleus accumbens medium spiny neuron subtypes in depression. Biol. Psychiatry 81, 645-653. doi:10.1016/j.biopsych.2016.09.007

Freeman, J.B., Dale, R., 2013. Assessing bimodality to detect the presence of a dual cognitive process. Behav. Res. Methods 45, 83-97. doi:10.3758/s13428-012-0225-x

Friedman, A.K., Juarez, B., Ku, S.M., Zhang, H., Calizo, R.C., Walsh, J.J., Chaudhury, D., Zhang, S., Hawkins, A., Dietz, D.M., Murrough, J.W., Ribadeneira, M., Wong, E.H., Neve, R.L., Han, M.-H., 2016. KCNQ channel openers reverse depressive symptoms via an active resilience mechanism. Nat. Commun. 7, 11671. doi:10.1038/ncomms11671

Friedman, A.K., Walsh, J.J., Juarez, B., Ku, S.M., Chaudhury, D., Wang, J., Li, X., Dietz, D.M., Pan, N., Vialou, V.F., Neve, R.L., Yue, Z., Han, M.-H., 2014. Enhancing depression mechanisms in midbrain dopamine neurons achieves homeostatic resilience. Science 344, 313-319. doi:10.1126/science.1249240

Fujisawa, S., Buzsáki, G., 2011. A 4 Hz oscillation adaptively synchronizes prefrontal, VTA, and hippocampal activities. Neuron 72, 153-165. doi:10.1016/j.neuron.2011.08.018

Fujiyama, F., Sohn, J., Nakano, T., Furuta, T., Nakamura, K.C., Matsuda, W., Kaneko, T., 2011. Exclusive 
and common targets of neostriatofugal projections of rat striosome neurons: a single neurontracing study using a viral vector. Eur. J. Neurosci. 33, 668-677. doi:10.1111/j.1460-

9568.2010.07564.x

Fuxe, K., Dahlström, A.B., Jonsson, G., Marcellino, D., Guescini, M., Dam, M., Manger, P., Agnati, L., 2010. The discovery of central monoamine neurons gave volume transmission to the wired brain. Prog. Neurobiol. 90, 82-100. doi:10.1016/j.pneurobio.2009.10.012

Galtieri, D.J., Estep, C.M., Wokosin, D.L., Traynelis, S., Surmeier, D.J., 2017. Pedunculopontine glutamatergic neurons control spike patterning in substantia nigra dopaminergic neurons. Elife 6. doi:10.7554/eLife.30352

Gantz, S.C., Ford, C.P., Morikawa, H., Williams, J.T., 2018. The evolving understanding of dopamine neurons in the substantia nigra and ventral tegmental area. Annu. Rev. Physiol. 80, 219-241. doi:10.1146/annurev-physiol-021317-121615

Gantz, S.C., Robinson, B.G., Buck, D.C., Bunzow, J.R., Neve, R.L., Williams, J.T., Neve, K.A., 2015. Distinct regulation of dopamine D2S and D2L autoreceptor signaling by calcium. Elife 4. doi:10.7554/eLife.09358

Geffen, L.B., Jessell, T.M., Cuello, A.C., Iversen, L.L., 1976. Release of dopamine from dendrites in rat substantia nigra. Nature 260, 258-260. doi:10.1038/260258a0

Geisler, S., Zahm, D.S., 2005. Afferents of the ventral tegmental area in the rat-anatomical substratum for integrative functions. J. Comp. Neurol. 490, 270-294. doi:10.1002/cne.20668

Gerfen, C.R., 2000. Molecular effects of dopamine on striatal-projection pathways. Trends Neurosci. 23, S64-70. doi:10.1016/s1471-1931(00)00019-7

Gerfen, C.R., Engber, T.M., Mahan, L.C., Susel, Z., Chase, T.N., Monsma, F.J., Sibley, D.R., 1990. D1 and D2 dopamine receptor-regulated gene expression of striatonigral and striatopallidal neurons. Science 250, 1429-1432. doi:10.1126/science.2147780

Gerfen, C.R., Surmeier, D.J., 2011. Modulation of striatal projection systems by dopamine. Annu. Rev. Neurosci. 34, 441-466. doi:10.1146/annurev-neuro-061010-113641

German, D.C., Manaye, K.F., 1993. Midbrain dopaminergic neurons (nuclei A8, A9, and A10): threedimensional reconstruction in the rat. J. Comp. Neurol. 331, 297-309. doi:10.1002/cne.903310302

Goetz, C.G., 2011. The history of Parkinson's disease: early clinical descriptions and neurological therapies. Cold Spring Harb. Perspect. Med. 1, a008862. doi:10.1101/cshperspect.a008862

Goto, Y., Grace, A.A., 2005. Dopaminergic modulation of limbic and cortical drive of nucleus accumbens in goal-directed behavior. Nat. Neurosci. 8, 805-812. doi:10.1038/nn1471

Grace, A.A., 1991. Phasic versus tonic dopamine release and the modulation of dopamine system responsivity: a hypothesis for the etiology of schizophrenia. Neuroscience 41, 1-24. doi:10.1016/0306-4522(91)90196-u

Grace, A.A., 2016. Dysregulation of the dopamine system in the pathophysiology of schizophrenia and depression. Nat. Rev. Neurosci. 17, 524-532. doi:10.1038/nrn.2016.57

Grace, A.A., Bunney, B.S., 1980. Nigral dopamine neurons: intracellular recording and identification with L-dopa injection and histofluorescence. Science 210, 654-656. doi:10.1126/science.7433992

Grace, A.A., Bunney, B.S., 1983a. Intracellular and extracellular electrophysiology of nigral dopaminergic neurons--2. Action potential generating mechanisms and morphological correlates. Neuroscience 10, 317-331. doi:10.1016/0306-4522(83)90136-7

Grace, A.A., Bunney, B.S., 1983b. Intracellular and extracellular electrophysiology of nigral dopaminergic neurons--1. Identification and characterization. Neuroscience 10, 301-315. doi:10.1016/03064522(83)90135-5

Grace, A.A., Bunney, B.S., 1983c. Intracellular and extracellular electrophysiology of nigral dopaminergic neurons--3. Evidence for electrotonic coupling. Neuroscience 10, 333-348. doi:10.1016/03064522(83)90137-9

Grace, A.A., Bunney, B.S., 1984a. The control of firing pattern in nigral dopamine neurons: single spike firing. J. Neurosci. 4, 2866-2876.

Grace, A.A., Bunney, B.S., 1984b. The control of firing pattern in nigral dopamine neurons: burst firing. J. Neurosci. 4, 2877-2890. doi:10.1523/JNEUROSCI.04-11-02877.1984

Grace, A.A., Onn, S.P., 1989. Morphology and electrophysiological properties of immunocytochemically 
identified rat dopamine neurons recorded in vitro. J. Neurosci. 9, 3463-3481. doi:10.1523/JNEUROSCI.09-10-03463.1989

Grillner, S., Robertson, B., 2016. The basal ganglia over 500 million years. Curr. Biol. 26, R1088-R1100. doi:10.1016/j.cub.2016.06.041

Groves, P.M., Linder, J.C., 1983. Dendro-dendritic synapses in substantia nigra: descriptions based on analysis of serial sections. Exp. Brain Res. 49, 209-217. doi:10.1007/BF00238581

Gunaydin, L.A., Kreitzer, A.C., 2016. Cortico-Basal Ganglia Circuit Function in Psychiatric Disease. Annu. Rev. Physiol. 78, 327-350. doi:10.1146/annurev-physiol-021115-105355

Haber, S.N., 2014. The place of dopamine in the cortico-basal ganglia circuit. Neuroscience 282, $248-257$. doi:10.1016/j.neuroscience.2014.10.008

Haber, S.N., Fudge, J.L., McFarland, N.R., 2000. Striatonigrostriatal pathways in primates form an ascending spiral from the shell to the dorsolateral striatum. J. Neurosci. 20, 2369-2382.

Halliday, G.M., Törk, I., 1986. Comparative anatomy of the ventromedial mesencephalic tegmentum in the rat, cat, monkey and human. J. Comp. Neurol. 252, 423-445. doi:10.1002/cne.902520402

Hampp, G., Ripperger, J.A., Houben, T., Schmutz, I., Blex, C., Perreau-Lenz, S., Brunk, I., Spanagel, R., Ahnert-Hilger, G., Meijer, J.H., Albrecht, U., 2008. Regulation of monoamine oxidase A by circadian-clock components implies clock influence on mood. Curr. Biol. 18, 678-683. doi:10.1016/j.cub.2008.04.012

Hansen, H.H., Waroux, O., Seutin, V., Jentsch, T.J., Aznar, S., Mikkelsen, J.D., 2008. Kv7 channels: interaction with dopaminergic and serotonergic neurotransmission in the CNS. J. Physiol. (Lond.) 586, 1823-1832. doi:10.1113/jphysiol.2007.149450

Häusser, M., Stuart, G., Racca, C., Sakmann, B., 1995. Axonal initiation and active dendritic propagation of action potentials in substantia nigra neurons. Neuron 15, 637-647. doi:10.1016/08966273(95)90152-3

Higgs, M.H., Spain, W.J., 2011. Kv1 channels control spike threshold dynamics and spike timing in cortical pyramidal neurones. J. Physiol. (Lond.) 589, 5125-5142. doi:10.1113/jphysiol.2011.216721

Hikosaka, O., Ghazizadeh, A., Griggs, W., Amita, H., 2018. Parallel basal ganglia circuits for decision making. J. Neural Transm. 125, 515-529. doi:10.1007/s00702-017-1691-1

Hladky, S.B., Haydon, D.A., 1984. Ion movements in gramicidin channels, in: Ion Channels: Molecular and Physiological Aspects, Current Topics in Membranes and Transport. Elsevier, pp. 327-372. doi:10.1016/S0070-2161(08)60444-X

Hökfelt, T., Johansson, O., Goldstein, M., 1984a. Central catecholamine neurons as revealed by immunohistochemistry with special reference to adrenaline neurons, in: Björklund, A., Hökfelt, T. (Eds.), Handbook of Chemical Neuroanatomy, Vol 2. Classical Transmitters in the CNS, Part I. Elsevier, pp. 157-276.

Hökfelt, T., Martensson, R., Björklund, A., Kleinau, S., Goldstein, M., 1984b. Distribution maps of tyrosinehydroxylase-immunoreactive neurons in the rat brain, in: Björklund, A., Hökfelt, T. (Eds.), Handbook of Chemical Neuroanatomy, Vol 2. Classical Transmitters in the CNS, Part I. Elsevier, pp. 277-379.

Hollerman, J.R., Schultz, W., 1998. Dopamine neurons report an error in the temporal prediction of reward during learning. Nat. Neurosci. 1, 304-309. doi:10.1038/1124

Hood, S., Cassidy, P., Cossette, M.-P., Weigl, Y., Verwey, M., Robinson, B., Stewart, J., Amir, S., 2010. Endogenous dopamine regulates the rhythm of expression of the clock protein PER2 in the rat dorsal striatum via daily activation of D2 dopamine receptors. J. Neurosci. 30, 14046-14058. doi:10.1523/JNEUROSCI.2128-10.2010

Hornykiewicz, O., 2002. Dopamine miracle: from brain homogenate to dopamine replacement. Mov. Disord. 17, 501-508. doi:10.1002/mds.10115

Howe, M.W., Dombeck, D.A., 2016. Rapid signalling in distinct dopaminergic axons during locomotion and reward. Nature 535, 505-510. doi:10.1038/nature18942

Howes, O.D., McCutcheon, R., Owen, M.J., Murray, R.M., 2017. The role of genes, stress, and dopamine in the development of schizophrenia. Biol. Psychiatry 81, 9-20. doi:10.1016/j.biopsych.2016.07.014

Hu, W., Tian, C., Li, T., Yang, M., Hou, H., Shu, Y., 2009. Distinct contributions of Na(v)1.6 and Na(v) 1.2 in action potential initiation and backpropagation. Nat. Neurosci. 12, 996-1002. 
doi:10.1038/nn.2359

Huang, C., Resnik, A., Celikel, T., Englitz, B., 2016. Adaptive spike threshold enables robust and temporally precise neuronal encoding. PLoS Comput. Biol. 12, e1004984. doi:10.1371/journal.pcbi.1004984

Huang, Q., Zhou, D., DiFiglia, M., 1992. Neurobiotin, a useful neuroanatomical tracer for in vivo anterograde, retrograde and transneuronal tract-tracing and for in vitro labeling of neurons. J. Neurosci. Methods 41, 31-43. doi:10.1016/0165-0270(92)90121-s

Humphries, M.D., Prescott, T.J., 2010. The ventral basal ganglia, a selection mechanism at the crossroads of space, strategy, and reward. Prog. Neurobiol. 90, 385-417. doi:10.1016/j.pneurobio.2009.11.003

Hyland, B.I., Reynolds, J.N.J., Hay, J., Perk, C.G., Miller, R., 2002. Firing modes of midbrain dopamine cells in the freely moving rat. Neuroscience 114, 475-492. doi:10.1016/s0306-4522(02)00267-1

Ikemoto, S., 2007. Dopamine reward circuitry: two projection systems from the ventral midbrain to the nucleus accumbens-olfactory tubercle complex. Brain Res. Rev. 56, 27-78. doi:10.1016/j.brainresrev.2007.05.004

Ikemoto, S., Yang, C., Tan, A., 2015. Basal ganglia circuit loops, dopamine and motivation: A review and enquiry. Behav. Brain Res. 290, 17-31. doi:10.1016/j.bbr.2015.04.018

Iyer, R., Ungless, M.A., Faisal, A.A., 2017. Calcium-activated SK channels control firing regularity by modulating sodium channel availability in midbrain dopamine neurons. Sci. Rep. 7, 5248. doi:10.1038/s41598-017-05578-5

Jackson, D.M., Westlind-Danielsson, A., 1994. Dopamine receptors: molecular biology, biochemistry and behavioural aspects. Pharmacol. Ther. 64, 291-370. doi:10.1016/0163-7258(94)90041-8

Jang, J.Y., Jang, M., Kim, S.H., Um, K.B., Kang, Y.K., Kim, H.J., Chung, S., Park, M.K., 2011. Regulation of dopaminergic neuron firing by heterogeneous dopamine autoreceptors in the substantia nigra pars compacta. J. Neurochem. 116, 966-974. doi:10.1111/j.1471-4159.2010.07107.x

Johnson, S.W., Seutin, V., North, R.A., 1992. Burst firing in dopamine neurons induced by N-methyl-Daspartate: role of electrogenic sodium pump. Science 258, 665-667. doi:10.1126/science.1329209

Jonkman, J., Brown, C.M., 2015. Any Way You Slice It-A Comparison of Confocal Microscopy Techniques. J Biomol Tech 26, 54-65. doi:10.7171/jbt.15-2602-003

Juraska, J.M., Wilson, C.J., Groves, P.M., 1977. The substantia nigra of the rat: a Golgi study. J. Comp. Neurol. 172, 585-600. doi:10.1002/cne.901720403

Käenmäki, M., Tammimäki, A., Myöhänen, T., Pakarinen, K., Amberg, C., Karayiorgou, M., Gogos, J.A. Männistö, P.T., 2010. Quantitative role of COMT in dopamine clearance in the prefrontal cortex of freely moving mice. J. Neurochem. 114, 1745-1755. doi:10.1111/j.1471-4159.2010.06889.x

Kandel, E.R., 2013. Principles Of Neural Science, 5th ed. Mcgraw-hill Medical, New York.

Kang, Y., Kitai, S.T., 1993. Calcium spike underlying rhythmic firing in dopaminergic neurons of the rat substantia nigra. Neurosci. Res. 18, 195-207. doi:10.1016/0168-0102(93)90055-u

Kang, Y.-J., Noh, Y., 2019. Development of Hartigan's Dip Statistic with Bimodality Coefficient to Assess Multimodality of Distributions. Mathematical Problems in Engineering 2019, 1-17. doi:10.1155/2019/4819475

Kawaguchi, Y., 1997. Neostriatal cell subtypes and their functional roles. Neurosci. Res. 27, 1-8. doi:10.1016/S0168-0102(96)01134-0

Kemp, J.M., Powell, T.P., 1971. The structure of the caudate nucleus of the cat: light and electron microscopy. Philos. Trans. R. Soc. Lond. B, Biol. Sci. 262, 383-401. doi:10.1098/rstb.1971.0102

Kesby, J.P., Eyles, D.W., McGrath, J.J., Scott, J.G., 2018. Dopamine, psychosis and schizophrenia: the widening gap between basic and clinical neuroscience. Transl. Psychiatry 8, 30. doi:10.1038/s41398-017-0071-9

Khaliq, Z.M., Bean, B.P., 2008. Dynamic, nonlinear feedback regulation of slow pacemaking by A-type potassium current in ventral tegmental area neurons. J. Neurosci. 28, 10905-10917. doi:10.1523/JNEUROSCI.2237-08.2008

Khaliq, Z.M., Bean, B.P., 2010. Pacemaking in dopaminergic ventral tegmental area neurons: depolarizing drive from background and voltage-dependent sodium conductances. J. Neurosci. 30, 74017413. doi:10.1523/JNEUROSCI.0143-10.2010

Kim, J.-I., Ganesan, S., Luo, S.X., Wu, Y.-W., Park, E., Huang, E.J., Chen, L., Ding, J.B., 2015. Aldehyde 
dehydrogenase 1a1 mediates a GABA synthesis pathway in midbrain dopaminergic neurons. Science 350, 102-106. doi:10.1126/science.aac4690

Kita, T., Kita, H., Kitai, S.T., 1986. Electrical membrane properties of rat substantia nigra compacta neurons in an in vitro slice preparation. Brain Res. 372, 21-30. doi:10.1016/0006-8993(86)91454$x$

Klitenick, M.A., Deutch, A.Y., Churchill, L., Kalivas, P.W., 1992. Topography and functional role of dopaminergic projections from the ventral mesencephalic tegmentum to the ventral pallidum. Neuroscience 50, 371-386. doi:10.1016/0306-4522(92)90430-a

Knowlton, C., Kutterer, S., Roeper, J., Canavier, C.C., 2018. Calcium dynamics control K-ATP channelmediated bursting in substantia nigra dopamine neurons: a combined experimental and modeling study. J. Neurophysiol. 119, 84-95. doi:10.1152/jn.00351.2017

Köhler, M., Hirschberg, B., Bond, C.T., Kinzie, J.M., Marrion, N.V., Maylie, J., Adelman, J.P., 1996. Smallconductance, calcium-activated potassium channels from mammalian brain. Science $273,1709-$ 1714. doi:10.1126/science.273.5282.1709

Koob, G.F., Volkow, N.D., 2016. Neurobiology of addiction: a neurocircuitry analysis. Lancet Psychiatry 3, 760-773. doi:10.1016/S2215-0366(16)00104-8

Kosaka, T., Hama, K., Nagatsu, I., 1987. Tyrosine hydroxylase-immunoreactive intrinsic neurons in the rat cerebral cortex. Exp. Brain Res. 68, 393-405. doi:10.1007/BF00248804

Kotecki, L., Hearing, M., McCall, N.M., Marron Fernandez de Velasco, E., Pravetoni, M., Arora, D., Victoria, N.C., Munoz, M.B., Xia, Z., Slesinger, P.A., Weaver, C.D., Wickman, K., 2015. GIRK Channels Modulate Opioid-Induced Motor Activity in a Cell Type- and Subunit-Dependent Manner. J. Neurosci. 35, 7131-7142. doi:10.1523/JNEUROSCI.5051-14.2015

Koyama, S., Appel, S.B., 2006. A-type K+ current of dopamine and GABA neurons in the ventral tegmental area. J. Neurophysiol. 96, 544-554. doi:10.1152/jn.01318.2005

Kupchik, Y.M., Brown, R.M., Heinsbroek, J.A., Lobo, M.K., Schwartz, D.J., Kalivas, P.W., 2015. Coding the direct/indirect pathways by $\mathrm{D} 1$ and $\mathrm{D} 2$ receptors is not valid for accumbens projections. Nat. Neurosci. 18, 1230-1232. doi:10.1038/nn.4068

Kupchik, Y.M., Kalivas, P.W., 2017. The direct and indirect pathways of the nucleus accumbens are not what you think. Neuropsychopharmacology 42, 369-370. doi:10.1038/npp.2016.160

Lacey, M.G., Mercuri, N.B., North, R.A., 1987. Dopamine acts on D2 receptors to increase potassium conductance in neurones of the rat substantia nigra zona compacta. J. Physiol. (Lond.) 392, 397416. doi:10.1113/jphysiol.1987.sp016787

Lammel, S., Hetzel, A., Häckel, O., Jones, I., Liss, B., Roeper, J., 2008. Unique properties of mesoprefrontal neurons within a dual mesocorticolimbic dopamine system. Neuron $57,760-773$. doi:10.1016/j.neuron.2008.01.022

Lammel, S., Lim, B.K., Malenka, R.C., 2014. Reward and aversion in a heterogeneous midbrain dopamine system. Neuropharmacology 76 Pt B, 351-359. doi:10.1016/j.neuropharm.2013.03.019

Lanciego, J.L., Luquin, N., Obeso, J.A., 2012. Functional neuroanatomy of the basal ganglia. Cold Spring Harb. Perspect. Med. 2, a009621. doi:10.1101/cshperspect.a009621

Langdon, A.J., Sharpe, M.J., Schoenbaum, G., Niv, Y., 2018. Model-based predictions for dopamine. Curr. Opin. Neurobiol. 49, 1-7. doi:10.1016/j.conb.2017.10.006

Lee, C.R., Tepper, J.M., 2009. Basal ganglia control of substantia nigra dopaminergic neurons. J Neural Transm Suppl 71-90. doi:10.1007/978-3-211-92660-4_6

Lees, A.J., Selikhova, M., Andrade, L.A., Duyckaerts, C., 2008. The black stuff and Konstantin Nikolaevich Tretiakoff. Mov. Disord. 23, 777-783. doi:10.1002/mds.21855

Li, L., Sun, H., Ding, J., Niu, C., Su, M., Zhang, L., Li, Y., Wang, C., Gamper, N., Du, X., Zhang, H., 2017. Selective targeting of M-type potassium Kv 7.4 channels demonstrates their key role in the regulation of dopaminergic neuronal excitability and depression-like behaviour. Br. J. Pharmacol. 174, 4277-4294. doi:10.1111/bph.14026

Li, W.C., Soffe, S.R., Roberts, A., 2004. A direct comparison of whole cell patch and sharp electrodes by simultaneous recording from single spinal neurons in frog tadpoles. J. Neurophysiol. 92, 380386. doi:10.1152/jn.01238.2003

Linley, J.E., 2013. Perforated whole-cell patch-clamp recording. Methods Mol. Biol. 998, 149-157. doi:10.1007/978-1-62703-351-0_11 
Liss, B., Franz, O., Sewing, S., Bruns, R., Neuhoff, H., Roeper, J., 2001. Tuning pacemaker frequency of individual dopaminergic neurons by Kv4.3L and KChip3.1 transcription. EMBO J. 20, 5715-5724. doi:10.1093/emboj/20.20.5715

Liss, B., Haeckel, O., Wildmann, J., Miki, T., Seino, S., Roeper, J., 2005. K-ATP channels promote the differential degeneration of dopaminergic midbrain neurons. Nat. Neurosci. 8, 1742-1751. doi:10.1038/nn1570

Liss, B., Roeper, J., 2009. Ion channels and regulation of dopamine neuron activity, in: Iversen, L., Iversen, S., Dunnett, S., Bjorklund, A. (Eds.), Dopamine Handbook. Oxford University Press, pp. 118-138. doi:10.1093/acprof:oso/9780195373035.003.0009

Llinás, R.R., Steriade, M., 2006. Bursting of thalamic neurons and states of vigilance. J. Neurophysiol. 95, 3297-3308. doi:10.1152/jn.00166.2006

Lüthi, A., McCormick, D.A., 1998. H-current: properties of a neuronal and network pacemaker. Neuron 21, 9-12. doi:10.1016/s0896-6273(00)80509-7

Macpherson, T., Morita, M., Hikida, T., 2014. Striatal direct and indirect pathways control decisionmaking behavior. Front. Psychol. 5, 1301. doi:10.3389/fpsyg.2014.01301

Maia, T.V., Frank, M.J., 2011. From reinforcement learning models to psychiatric and neurological disorders. Nat. Neurosci. 14, 154-162. doi:10.1038/nn.2723

Manger, P.R., Fahringer, H.M., Pettigrew, J.D., Siegel, J.M., 2002. The distribution and morphological characteristics of catecholaminergic cells in the brain of monotremes as revealed by tyrosine hydroxylase immunohistochemistry. Brain Behav. Evol. 60, 298-314. doi:10.1159/000067193

Margolis, E.B., Hjelmstad, G.O., Fujita, W., Fields, H.L., 2014. Direct bidirectional $\mu$-opioid control of midbrain dopamine neurons. J. Neurosci. 34, 14707-14716. doi:10.1523/JNEUROSCI.214414.2014

Margrie, T.W., Brecht, M., Sakmann, B., 2002. In vivo, low-resistance, whole-cell recordings from neurons in the anaesthetized and awake mammalian brain. Pflugers Arch. 444, 491-498. doi:10.1007/s00424-002-0831-z

Marinelli, M., McCutcheon, J.E., 2014. Heterogeneity of dopamine neuron activity across traits and states. Neuroscience 282, 176-197. doi:10.1016/j.neuroscience.2014.07.034

Matsuda, W., Furuta, T., Nakamura, K.C., Hioki, H., Fujiyama, F., Arai, R., Kaneko, T., 2009. Single nigrostriatal dopaminergic neurons form widely spread and highly dense axonal arborizations in the neostriatum. J. Neurosci. 29, 444-453. doi:10.1523/JNEUROSCI.4029-08.2009

McCall, N.M., Kotecki, L., Dominguez-Lopez, S., Marron Fernandez de Velasco, E., Carlblom, N., Sharpe, A.L., Beckstead, M.J., Wickman, K., 2017. Selective ablation of GIRK channels in dopamine neurons alters behavioral effects of cocaine in mice. Neuropsychopharmacology 42, 707-715. doi:10.1038/npp.2016.138

McCall, N.M., Marron Fernandez de Velasco, E., Wickman, K., 2019. GIRK channel activity in dopamine neurons of the ventral tegmental area bidirectionally regulates behavioral sensitivity to cocaine. J. Neurosci. 39, 3600-3610. doi:10.1523/JNEUROSCI.3101-18.2019

McElvain, J.S., Schenk, J.O., 1992. A multisubstrate mechanism of striatal dopamine uptake and its inhibition by cocaine. Biochem. Pharmacol. 43, 2189-2199. doi:10.1016/0006-2952(92)90178-I

Menegas, W., Akiti, K., Amo, R., Uchida, N., Watabe-Uchida, M., 2018. Dopamine neurons projecting to the posterior striatum reinforce avoidance of threatening stimuli. Nat. Neurosci. 21, 1421-1430. doi:10.1038/s41593-018-0222-1

Miller, J.D., Sanghera, M.K., German, D.C., 1981. Mesencephalic dopaminergic unit activity in the behaviorally conditioned rat. Life Sci. 29, 1255-1263. doi:10.1016/0024-3205(81)90231-9

Mink, J.W., 1996. The basal ganglia: focused selection and inhibition of competing motor programs. Prog. Neurobiol. 50, 381-425. doi:10.1016/S0301-0082(96)00042-1

Mink, J.W., 2003. The Basal Ganglia and involuntary movements: impaired inhibition of competing motor patterns. Arch. Neurol. 60, 1365-1368. doi:10.1001/archneur.60.10.1365

Miranda-Barrientos, J., Chambers, I., Mongia, S., Liu, B., Wang, H.-L., Mateo-Semidey, G.E., Margolis, E.B., Zhang, S., Morales, M., 2021. Ventral Tegmental Area GABA, glutamate, and glutamate-GABA neurons are heterogeneous in their electrophysiological and pharmacological properties. Eur. J. Neurosci. doi:10.1111/ejn.15156

Missale, C., Nash, S.R., Robinson, S.W., Jaber, M., Caron, M.G., 1998. Dopamine receptors: from structure 
to function. Physiol. Rev. 78, 189-225. doi:10.1152/physrev.1998.78.1.189

Mitrovic, I., Napier, T.C., 2002. Mu and kappa opioid agonists modulate ventral tegmental area input to the ventral pallidum. Eur. J. Neurosci. 15, 257-268. doi:10.1046/j.0953-816x.2001.01860.x

Montagu, K.A., 1957. Catechol compounds in rat tissues and in brains of different animals. Nature 180, 244-245. doi:10.1038/180244a0

Morales, M., Margolis, E.B., 2017. Ventral tegmental area: cellular heterogeneity, connectivity and behaviour. Nat. Rev. Neurosci. 18, 73-85. doi:10.1038/nrn.2016.165

Mulvihill, K.G., 2019. Presynaptic regulation of dopamine release: Role of the DAT and VMAT2 transporters. Neurochem. Int. 122, 94-105. doi:10.1016/j.neuint.2018.11.004

Napier, T.C., 1992. Contribution of the amygdala and nucleus accumbens to ventral pallidal responses to dopamine agonists. Synapse 10, 110-119. doi:10.1002/syn.890100205

Nasser, H.M., Calu, D.J., Schoenbaum, G., Sharpe, M.J., 2017. The dopamine prediction error: contributions to associative models of reward learning. Front. Psychol. 8, 244. doi:10.3389/fpsyg.2017.00244

Naundorf, B., Wolf, F., Volgushev, M., 2006. Unique features of action potential initiation in cortical neurons. Nature 440, 1060-1063. doi:10.1038/nature04610

Nedergaard, S., 1999. Regulation of action potential size and excitability in substantia nigra compacta neurons: sensitivity to 4-aminopyridine. J. Neurophysiol. 82, 2903-2913. doi:10.1152/jn.1999.82.6.2903

Nedergaard, S., Greenfield, S.A., 1992. Sub-populations of pars compacta neurons in the substantia nigra: the significance of qualitatively and quantitatively distinct conductances. Neuroscience 48, 423437. doi:10.1016/0306-4522(92)90502-s

Nelson, E.L., Liang, C.L., Sinton, C.M., German, D.C., 1996. Midbrain dopaminergic neurons in the mouse: Computer-assisted mapping. J. Comp. Neurol. 369, 361-371. doi:10.1002/(SICI)10969861(19960603)369:3<361::AID-CNE3>3.0.CO;2-3

Nestler, E., Hyman, S., Malenka, R., 2015. Molecular Neuropharmacology: A Foundation for Clinical Neuroscience, Third Edition, 3rd ed. McGraw-Hill Education / Medical, New York.

Neuhoff, H., Neu, A., Liss, B., Roeper, J., 2002. I(h) channels contribute to the different functional properties of identified dopaminergic subpopulations in the midbrain. J. Neurosci. 22, 12901302.

Noguchi, A., Ikegaya, Y., Matsumoto, N., 2021. In Vivo Whole-Cell Patch-Clamp Methods: Recent Technical Progress and Future Perspectives. Sensors (Basel) 21. doi:10.3390/s21041448

Ntamati, N.R., Creed, M., Achargui, R., Lüscher, C., 2018. Periaqueductal efferents to dopamine and GABA neurons of the VTA. PLoS One 13, e0190297. doi:10.1371/journal.pone.0190297

Obeso, J.A., Rodriguez-Oroz, M.C., Goetz, C.G., Marin, C., Kordower, J.H., Rodriguez, M., Hirsch, E.C., Farrer, M., Schapira, A.H.V., Halliday, G., 2010. Missing pieces in the Parkinson's disease puzzle. Nat. Med. 16, 653-661. doi:10.1038/nm.2165

Olds, J., Milner, P., 1954. Positive reinforcement produced by electrical stimulation of septal area and other regions of rat brain. J Comp Physiol Psychol 47, 419-427. doi:10.1037/h0058775

Olson, V.G., Nestler, E.J., 2007. Topographical organization of GABAergic neurons within the ventral tegmental area of the rat. Synapse 61, 87-95. doi:10.1002/syn.20345

Overton, P.G., Clark, D., 1997. Burst firing in midbrain dopaminergic neurons. Brain Res Brain Res Rev 25, 312-334. doi:10.1016/S0165-0173(97)00039-8

Paladini, C.A., Celada, P., Tepper, J.M., 1999. Striatal, pallidal, and pars reticulata evoked inhibition of nigrostriatal dopaminergic neurons is mediated by GABA(A) receptors in vivo. Neuroscience 89 , 799-812. doi:10.1016/s0306-4522(98)00355-8

Paladini, C.A., Tepper, J.M., 1999. GABA(A) and GABA(B) antagonists differentially affect the firing pattern of substantia nigra dopaminergic neurons in vivo. Synapse 32, 165-176. doi:10.1002/(SICI)10982396(19990601)32:3<165::AID-SYN3>3.0.CO;2-N

Parent, A., 2012. The history of the basal ganglia: the contribution of karl friedrich burdach. NM 03, 374379. doi:10.4236/nm.2012.34046

Parent, A., Bouchard, C., Smith, Y., 1984. The striatopallidal and striatonigral projections: two distinct fiber systems in primate. Brain Res. 303, 385-390. doi:10.1016/0006-8993(84)91224-1

Parent, A., Hazrati, L.N., 1995. Functional anatomy of the basal ganglia. I. The cortico-basal ganglia- 
thalamo-cortical loop. Brain Res Brain Res Rev 20, 91-127. doi:10.1016/0165-0173(94)00007-C

Parent, A., Sato, F., Wu, Y., Gauthier, J., Lévesque, M., Parent, M., 2000. Organization of the basal ganglia: the importance of axonal collateralization. Trends Neurosci. 23, S20-7. doi:10.1016/s14711931(00)00022-7

Paxinos, G., Franklin, K., 2007. The Mouse Brain in Stereotaxic Coordinates, 3rd ed. Academic Press.

Peris, J., MacFadyen, K., Smith, J.A., de Kloet, A.D., Wang, L., Krause, E.G., 2017. Oxytocin receptors are expressed on dopamine and glutamate neurons in the mouse ventral tegmental area that project to nucleus accumbens and other mesolimbic targets. J. Comp. Neurol. 525, 1094-1108. doi:10.1002/cne.24116

Persson, S., Havton, L.A., 2009. Retrogradely transported fluorogold accumulates in lysosomes of neurons and is detectable ultrastructurally using post-embedding immuno-gold methods. J. Neurosci. Methods 184, 42-47. doi:10.1016/j.jneumeth.2009.07.017

Petrelli, F., Dallérac, G., Pucci, L., Calì, C., Zehnder, T., Sultan, S., Lecca, S., Chicca, A., Ivanov, A., Asensio, C.S., Gundersen, V., Toni, N., Knott, G.W., Magara, F., Gertsch, J., Kirchhoff, F., Déglon, N., Giros, B., Edwards, R.H., Mothet, J.-P., Bezzi, P., 2020. Dysfunction of homeostatic control of dopamine by astrocytes in the developing prefrontal cortex leads to cognitive impairments. Mol. Psychiatry 25, 732-749. doi:10.1038/s41380-018-0226-y

Pfister, R., Schwarz, K.A., Janczyk, M., Dale, R., Freeman, J.B., 2013. Good things peak in pairs: a note on the bimodality coefficient. Front. Psychol. 4, 700. doi:10.3389/fpsyg.2013.00700

Philippart, F., Destreel, G., Merino-Sepúlveda, P., Henny, P., Engel, D., Seutin, V., 2016. Differential somatic ca2+ channel profile in midbrain dopaminergic neurons. J. Neurosci. 36, 7234-7245. doi:10.1523/JNEUROSCI.0459-16.2016

Pickel, V.M., Beckley, S.C., Joh, T.H., Reis, D.J., 1981. Ultrastructural immunocytochemical localization of tyrosine hydroxylase in the neostriatum. Brain Res. 225, 373-385. doi:10.1016/00068993(81)90843-x

Pinault, D., 1996. A novel single-cell staining procedure performed in vivo under electrophysiological control: morpho-functional features of juxtacellularly labeled thalamic cells and other central neurons with biocytin or Neurobiotin. J. Neurosci. Methods 65, 113-136. doi:10.1016/01650270(95)00144-1

Platkiewicz, J., Brette, R., 2011. Impact of fast sodium channel inactivation on spike threshold dynamics and synaptic integration. PLoS Comput. Biol. 7, e1001129. doi:10.1371/journal.pcbi.1001129

Poulin, J.-F., Caronia, G., Hofer, C., Cui, Q., Helm, B., Ramakrishnan, C., Chan, C.S., Dombeck, D.A., Deisseroth, K., Awatramani, R., 2018. Mapping projections of molecularly defined dopamine neuron subtypes using intersectional genetic approaches. Nat. Neurosci. 21, 1260-1271. doi:10.1038/s41593-018-0203-4

Rescorla, R.A., Wagner, A.R., 1972. A theory of Pavlovian conditioning: Variations in the effectiveness of reinforcement and nonreinforcement., in: Classical Conditioning II: Current Research and Theory. Appleton-Century-Crofts, New York.

Rice, M.E., Cragg, S.J., 2008. Dopamine spillover after quantal release: rethinking dopamine transmission in the nigrostriatal pathway. Brain Res. Rev. 58, 303-313. doi:10.1016/j.brainresrev.2008.02.004

Rice, M.E., Patel, J.C., 2015. Somatodendritic dopamine release: recent mechanistic insights. Philos. Trans. R. Soc. Lond. B, Biol. Sci. 370. doi:10.1098/rstb.2014.0185

Richards, C.D., Shiroyama, T., Kitai, S.T., 1997. Electrophysiological and immunocytochemical characterization of GABA and dopamine neurons in the substantia nigra of the rat. Neuroscience 80, 545-557. doi:10.1016/s0306-4522(97)00093-6

Richfield, E.K., Penney, J.B., Young, A.B., 1989. Anatomical and affinity state comparisons between dopamine D1 and D2 receptors in the rat central nervous system. Neuroscience 30, 767-777. doi:10.1016/0306-4522(89)90168-1

Rifkin, R.A., Moss, S.J., Slesinger, P.A., 2017. G Protein-Gated Potassium Channels: A Link to Drug Addiction. Trends Pharmacol. Sci. 38, 378-392. doi:10.1016/j.tips.2017.01.007

Root, D.H., Melendez, R.I., Zaborszky, L., Napier, T.C., 2015. The ventral pallidum: Subregion-specific functional anatomy and roles in motivated behaviors. Prog. Neurobiol. 130, 29-70. doi:10.1016/j.pneurobio.2015.03.005

Salamone, J.D., Correa, M., 2012. The mysterious motivational functions of mesolimbic dopamine. 
Neuron 76, 470-485. doi:10.1016/j.neuron.2012.10.021

Sanghera, M.K., Trulson, M.E., German, D.C., 1984. Electrophysiological properties of mouse dopamine neurons: in vivo and in vitro studies. Neuroscience 12, 793-801. doi:10.1016/03064522(84)90171-4

Schiemann, J., Schlaudraff, F., Klose, V., Bingmer, M., Seino, S., Magill, P.J., Zaghloul, K.A., Schneider, G., Liss, B., Roeper, J., 2012. K-ATP channels in dopamine substantia nigra neurons control bursting and novelty-induced exploration. Nat. Neurosci. 15, 1272-1280. doi:10.1038/nn.3185

Schmued, L.C., Kyriakidis, K., Fallon, J.H., Ribak, C.E., 1989. Neurons containing retrogradely transported Fluoro-Gold exhibit a variety of lysosomal profiles: a combined brightfield, fluorescence, and electron microscopic study. J Neurocytol 18, 333-343. doi:10.1007/BF01190836

Schultz, W., 2007. Behavioral dopamine signals. Trends Neurosci. 30, 203-210. doi:10.1016/j.tins.2007.03.007

Schultz, W., 2019. Recent advances in understanding the role of phasic dopamine activity. [version 1; peer review: 3 approved]. F1000Res. 8. doi:10.12688/f1000research.19793.1

Schultz, W., Dayan, P., Montague, P.R., 1997. A neural substrate of prediction and reward. Science 275, 1593-1599. doi:10.1126/science.275.5306.1593

Segev, D., Korngreen, A., 2007. Kinetics of two voltage-gated K+ conductances in substantia nigra dopaminergic neurons. Brain Res. 1173, 27-35. doi:10.1016/j.brainres.2007.08.006

Seutin, V., Engel, D., 2010. Differences in $\mathrm{Na}+$ conductance density and $\mathrm{Na}+$ channel functional properties between dopamine and GABA neurons of the rat substantia nigra. J. Neurophysiol. 103, 30993114. doi:10.1152/jn.00513.2009

Sharpe, A.L., Varela, E., Bettinger, L., Beckstead, M.J., 2014. Methamphetamine self-administration in mice decreases GIRK channel-mediated currents in midbrain dopamine neurons. Int. J. Neuropsychopharmacol. 18. doi:10.1093/ijnp/pyu073

Sharpe, M.J., Chang, C.Y., Liu, M.A., Batchelor, H.M., Mueller, L.E., Jones, J.L., Niv, Y., Schoenbaum, G., 2017. Dopamine transients are sufficient and necessary for acquisition of model-based associations. Nat. Neurosci. 20, 735-742. doi:10.1038/nn.4538

Shepard, P.D., Bunney, B.S., 1991. Repetitive firing properties of putative dopamine-containing neurons in vitro: regulation by an apamin-sensitive $\mathrm{Ca}(2+)$-activated $\mathrm{K}+$ conductance. Exp. Brain Res. 86, 141-150. doi:10.1007/BF00231048

Shi, W.-X., 2009. Electrophysiological characteristics of dopamine neurons: a 35-year update. J Neural Transm Suppl 103-119. doi:10.1007/978-3-211-92660-4_8

Sidor, M.M., Spencer, S.M., Dzirasa, K., Parekh, P.K., Tye, K.M., Warden, M.R., Arey, R.N., Enwright, J.F., Jacobsen, J.P.R., Kumar, S., Remillard, E.M., Caron, M.G., Deisseroth, K., McClung, C.A., 2015. Daytime spikes in dopaminergic activity drive rapid mood-cycling in mice. Mol. Psychiatry 20, 1406-1419. doi:10.1038/mp.2014.167

Smith, Y., Kieval, J.Z., 2000. Anatomy of the dopamine system in the basal ganglia. Trends Neurosci. 23, S28-33. doi:10.1016/s1471-1931(00)00023-9

Stauffer, W.R., Lak, A., Yang, A., Borel, M., Paulsen, O., Boyden, E.S., Schultz, W., 2016. Dopamine Neuron-Specific Optogenetic Stimulation in Rhesus Macaques. Cell 166, 1564-1571.e6. doi:10.1016/j.cell.2016.08.024

Steinberg, E.E., Keiflin, R., Boivin, J.R., Witten, I.B., Deisseroth, K., Janak, P.H., 2013. A causal link between prediction errors, dopamine neurons and learning. Nat. Neurosci. 16, 966-973. doi:10.1038/nn.3413

Steinfels, G.F., Heym, J., Jacobs, B.L., 1981. Single unit activity of dopaminergic neurons in freely moving cats. Life Sci. 29, 1435-1442. doi:10.1016/0024-3205(81)90007-2

Su, M., Li, L., Wang, J., Sun, H., Zhang, L., Zhao, C., Xie, Y., Gamper, N., Du, X., Zhang, H., 2019. Kv7.4 Channel Contribute to Projection-Specific Auto-Inhibition of Dopamine Neurons in the Ventral Tegmental Area. Front. Cell Neurosci. 13, 557. doi:10.3389/fncel.2019.00557

Sulzer, D., Joyce, M.P., Lin, L., Geldwert, D., Haber, S.N., Hattori, T., Rayport, S., 1998. Dopamine neurons make glutamatergic synapses in vitro. J. Neurosci. 18, 4588-4602.

Sutton, R.S., Barto, A.G., 1981. Toward a modern theory of adaptive networks: expectation and prediction. Psychol. Rev. 88, 135-170. doi:10.1037/0033-295X.88.2.135

Tao, C., Zhang, G., Xiong, Y., Zhou, Y., 2015. Functional dissection of synaptic circuits: in vivo patch-clamp 
recording in neuroscience. Front. Neural Circuits 9, 23. doi:10.3389/fncir.2015.00023

Tapia, M., Baudot, P., Formisano-Tréziny, C., Dufour, M.A., Temporal, S., Lasserre, M., Marquèze-Pouey, B., Gabert, J., Kobayashi, K., Goaillard, J.-M., 2018. Neurotransmitter identity and electrophysiological phenotype are genetically coupled in midbrain dopaminergic neurons. Sci. Rep. 8, 13637. doi:10.1038/s41598-018-31765-z

Tarfa, R.A., Evans, R.C., Khaliq, Z.M., 2017. Enhanced sensitivity to hyperpolarizing inhibition in mesoaccumbal relative to nigrostriatal dopamine neuron subpopulations. J. Neurosci. 37, 33113330. doi:10.1523/JNEUROSCI.2969-16.2017

Taverna, S., Ilijic, E., Surmeier, D.J., 2008. Recurrent collateral connections of striatal medium spiny neurons are disrupted in models of Parkinson's disease. J. Neurosci. 28, 5504-5512. doi:10.1523/JNEUROSCI.5493-07.2008

Tecuapetla, F., Patel, J.C., Xenias, H., English, D., Tadros, I., Shah, F., Berlin, J., Deisseroth, K., Rice, M.E., Tepper, J.M., Koos, T., 2010. Glutamatergic signaling by mesolimbic dopamine neurons in the nucleus accumbens. J. Neurosci. 30, 7105-7110. doi:10.1523/JNEUROSCI.0265-10.2010

Tepper, J.M., Lee, C.R., 2007. GABAergic control of substantia nigra dopaminergic neurons, in: Gaba and the Basal Ganglia - From Molecules to Systems, Progress in Brain Research. Elsevier, pp. 189208. doi:10.1016/S0079-6123(06)60011-3

Tepper, J.M., Martin, L.P., Anderson, D.R., 1995. GABAA receptor-mediated inhibition of rat substantia nigra dopaminergic neurons by pars reticulata projection neurons. J. Neurosci. 15, 3092-3103.

Tian, J., Huang, R., Cohen, J.Y., Osakada, F., Kobak, D., Machens, C.K., Callaway, E.M., Uchida, N., WatabeUchida, M., 2016. Distributed and mixed information in monosynaptic inputs to dopamine neurons. Neuron 91, 1374-1389. doi:10.1016/j.neuron.2016.08.018

Tripathy, S.J., Burton, S.D., Geramita, M., Gerkin, R.C., Urban, N.N., 2015. Brain-wide analysis of electrophysiological diversity yields novel categorization of mammalian neuron types. J. Neurophysiol. 113, 3474-3489. doi:10.1152/jn.00237.2015

Tritsch, N.X., Ding, J.B., Sabatini, B.L., 2012. Dopaminergic neurons inhibit striatal output through noncanonical release of GABA. Nature 490, 262-266. doi:10.1038/nature11466

Tritsch, N.X., Oh, W.-J., Gu, C., Sabatini, B.L., 2014. Midbrain dopamine neurons sustain inhibitory transmission using plasma membrane uptake of GABA, not synthesis. Elife 3, e01936. doi:10.7554/eLife.01936

Trutti, A.C., Mulder, M.J., Hommel, B., Forstmann, B.U., 2019. Functional neuroanatomical review of the ventral tegmental area. Neuroimage 191, 258-268. doi:10.1016/j.neuroimage.2019.01.062

Tsai, H.-C., Zhang, F., Adamantidis, A., Stuber, G.D., Bonci, A., de Lecea, L., Deisseroth, K., 2009. Phasic firing in dopaminergic neurons is sufficient for behavioral conditioning. Science 324, 1080-1084. doi:10.1126/science.1168878

Tucker, K.R., Huertas, M.A., Horn, J.P., Canavier, C.C., Levitan, E.S., 2012. Pacemaker rate and depolarization block in nigral dopamine neurons: a somatic sodium channel balancing act. J. Neurosci. 32, 14519-14531. doi:10.1523/JNEUROSCI.1251-12.2012

Ungless, M.A., Argilli, E., Bonci, A., 2010. Effects of stress and aversion on dopamine neurons: implications for addiction. Neurosci. Biobehav. Rev. 35, 151-156. doi:10.1016/j.neubiorev.2010.04.006

Ungless, M.A., Grace, A.A., 2012. Are you or aren't you? Challenges associated with physiologically identifying dopamine neurons. Trends Neurosci. 35, 422-430. doi:10.1016/j.tins.2012.02.003

Ungless, M.A., Magill, P.J., Bolam, J.P., 2004. Uniform inhibition of dopamine neurons in the ventral tegmental area by aversive stimuli. Science 303, 2040-2042. doi:10.1126/science.1093360

Usiello, A., Baik, J.H., Rougé-Pont, F., Picetti, R., Dierich, A., LeMeur, M., Piazza, P.V., Borrelli, E., 2000. Distinct functions of the two isoforms of dopamine D2 receptors. Nature 408, 199-203. doi:10.1038/35041572

Verwey, M., Dhir, S., Amir, S., 2016. Circadian influences on dopamine circuits of the brain: regulation of striatal rhythms of clock gene expression and implications for psychopathology and disease. [version 1; peer review: 2 approved]. F1000Res. 5. doi:10.12688/f1000research.9180.1

Vickstrom, C.R., Liu, X., Zhang, Y., Mu, L., Kelly, T.J., Yan, X., Hu, M.-M., Snarrenberg, S.T., Liu, Q.-S., 2020. T-Type Calcium Channels Contribute to Burst Firing in a Subpopulation of Medial Habenula Neurons. Eneuro 7. doi:10.1523/ENEURO.0201-20.2020 
Volkow, N.D., Fowler, J.S., Wang, G.J., Baler, R., Telang, F., 2009. Imaging dopamine's role in drug abuse and addiction. Neuropharmacology 56 Suppl 1, 3-8. doi:10.1016/j.neuropharm.2008.05.022

Walsh, K.B., 2011. Targeting GIRK channels for the development of new therapeutic agents. Front. Pharmacol. 2, 64. doi:10.3389/fphar.2011.00064

Watabe-Uchida, M., Eshel, N., Uchida, N., 2017. Neural circuitry of reward prediction error. Annu. Rev. Neurosci. 40, 373-394. doi:10.1146/annurev-neuro-072116-031109

Webb, I.C., Lehman, M.N., Coolen, L.M., 2015. Diurnal and circadian regulation of reward-related neurophysiology and behavior. Physiol. Behav. 143, 58-69. doi:10.1016/j.physbeh.2015.02.034

Wessendorf, M.W., 1991. Fluoro-Gold: composition, and mechanism of uptake. Brain Res. 553, 135-148. doi:10.1016/0006-8993(91)90241-m

Wichmann, T., DeLong, M.R., 1996. Functional and pathophysiological models of the basal ganglia. Curr. Opin. Neurobiol. 6, 751-758. doi:10.1016/S0959-4388(96)80024-9

Wilchek, M., Bayer, E.A., 1990. Introduction to avidin-biotin technology. Meth. Enzymol. 184, 5-13. doi:10.1016/0076-6879(90)84256-g

Wilcox, K.S., Gutnick, M.J., Christoph, G.R., 1988. Electrophysiological properties of neurons in the lateral habenula nucleus: an in vitro study. J. Neurophysiol. 59, 212-225. doi:10.1152/jn.1988.59.1.212

Woehler, A., Lin, K.-H., Neher, E., 2014. Calcium-buffering effects of gluconate and nucleotides, as determined by a novel fluorimetric titration method. J. Physiol. (Lond.) 592, 4863-4875. doi:10.1113/jphysiol.2014.281097

Wolfart, J., Neuhoff, H., Franz, O., Roeper, J., 2001. Differential expression of the small-conductance, calcium-activated potassium channel SK3 is critical for pacemaker control in dopaminergic midbrain neurons. J. Neurosci. 21, 3443-3456.

Wolfart, J., Roeper, J., 2002. Selective coupling of T-type calcium channels to SK potassium channels prevents intrinsic bursting in dopaminergic midbrain neurons. J. Neurosci. 22, 3404-3413. doi:20026345

Xia, X.M., Fakler, B., Rivard, A., Wayman, G., Johnson-Pais, T., Keen, J.E., Ishii, T., Hirschberg, B., Bond, C.T., Lutsenko, S., Maylie, J., Adelman, J.P., 1998. Mechanism of calcium gating in smallconductance calcium-activated potassium channels. Nature 395, 503-507. doi:10.1038/26758

Xiao, C., Zhou, C.-Y., Jiang, J.-H., Yin, C., 2020. Neural circuits and nicotinic acetylcholine receptors mediate the cholinergic regulation of midbrain dopaminergic neurons and nicotine dependence. Acta Pharmacol Sin 41, 1-9. doi:10.1038/s41401-019-0299-4

Xiao, L., Priest, M.F., Nasenbeny, J., Lu, T., Kozorovitskiy, Y., 2017. Biased oxytocinergic modulation of midbrain dopamine systems. Neuron 95, 368-384.e5. doi:10.1016/j.neuron.2017.06.003

Xu, W., Lipscombe, D., 2001. Neuronal Ca(V)1.3alpha(1) L-type channels activate at relatively hyperpolarized membrane potentials and are incompletely inhibited by dihydropyridines. J. Neurosci. 21, 5944-5951.

Yamaguchi, T., Wang, H.-L., Li, X., Ng, T.H., Morales, M., 2011. Mesocorticolimbic glutamatergic pathway. J. Neurosci. 31, 8476-8490. doi:10.1523/JNEUROSCI.1598-11.2011

Yang, J., Xiao, Y., Li, L., He, Q., Li, M., Shu, Y., 2019. Biophysical Properties of Somatic and Axonal VoltageGated Sodium Channels in Midbrain Dopaminergic Neurons. Front. Cell Neurosci. 13, 317. doi:10.3389/fncel.2019.00317

Yin, H.H., Knowlton, B.J., 2006. The role of the basal ganglia in habit formation. Nat. Rev. Neurosci. 7, 464-476. doi:10.1038/nrn1919

Yu, Y., Shu, Y., McCormick, D.A., 2008. Cortical action potential backpropagation explains spike threshold variability and rapid-onset kinetics. J. Neurosci. 28, 7260-7272. doi:10.1523/JNEUROSCI.161308.2008

Zhang, S., Qi, J., Li, X., Wang, H.-L., Britt, J.P., Hoffman, A.F., Bonci, A., Lupica, C.R., Morales, M., 2015. Dopaminergic and glutamatergic microdomains in a subset of rodent mesoaccumbens axons. Nat. Neurosci. 18, 386-392. doi:10.1038/nn.3945

Zhang, T.A., Placzek, A.N., Dani, J.A., 2010. In vitro identification and electrophysiological characterization of dopamine neurons in the ventral tegmental area. Neuropharmacology 59, 431-436. doi:10.1016/j.neuropharm.2010.06.004 
